\title{
The production of VLCPUFAs in plants
}

\author{
PhD Thesis \\ in partial fulfilment of the requirements \\ for the degree "Doctor rerum naturalium (Dr. rer. nat.)" \\ in the Molecular Biology Program \\ at the Georg August University Göttingen, \\ Faculty of Biology \\ submitted by \\ Katharina Ahmann \\ born in \\ Osnabrück, Germany
}





\section{Affidavit}

Herewith I affirm that I wrote this $\mathrm{PhD}$ Thesis independently and with no other sources and aids than quoted.

Katharina Ahmann

December 2010

Göttingen, Germany 


\section{List of Publications}

Journal article

Wagner M, Hoppe K, Czabany T, Heilmann M, Daum G, Feussner I, Fulda M (2010) Identification and characterization of an acyl-CoA:diacylglycerol acyltransferase 2 (DGAT2) gene from the microalga O. tauri. Plant Physiology and Biochemistry 48: $407-416$

In preparation

Ahmann K, Heilmann M, Feussner I. Identification of a $\Delta 4$-desaturase from the microalga Ostreococcus lucimarinus. 


\section{Table of Contents}

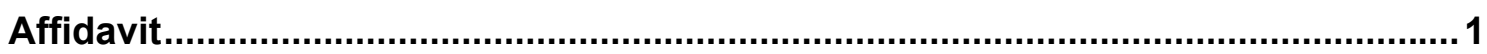

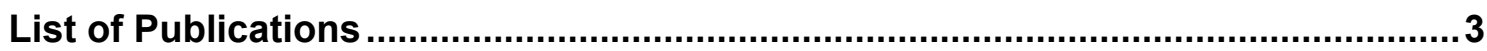

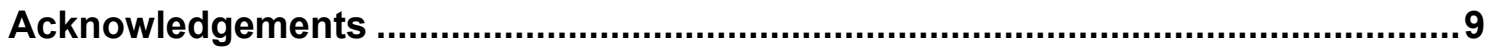

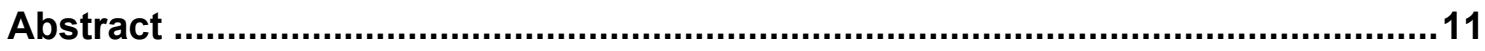

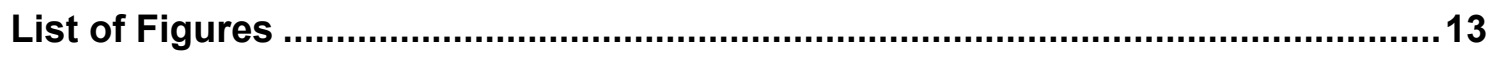

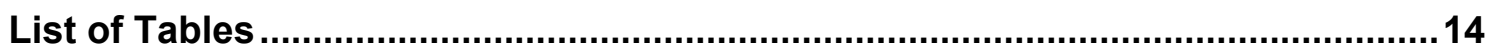

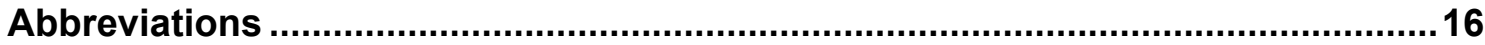

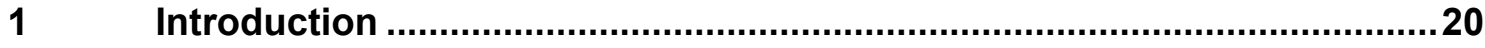

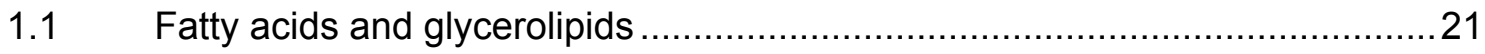

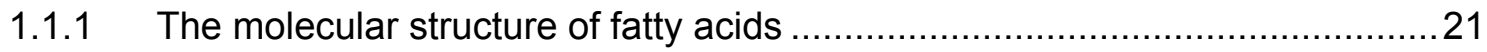

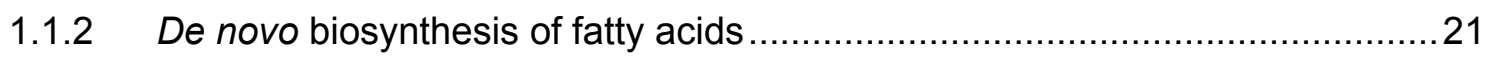

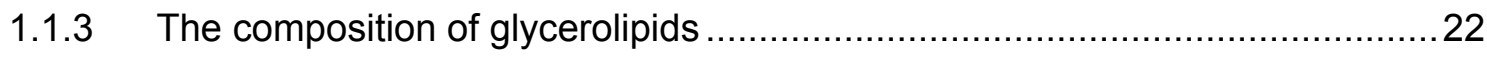

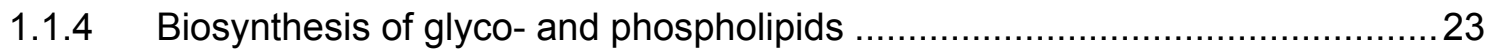

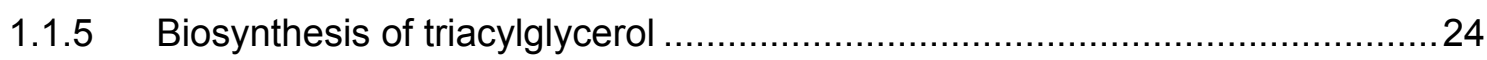

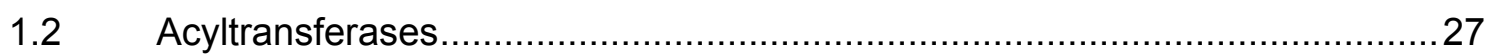

1.2.1 Acyl-CoA:lyso-phosphatidylcholine acyltransferases (LPCATs) ....................27

1.2.2 Acyl-CoA:diacylglycerol acyltransferases (DGATs) …................................27

1.2.3 Phospholipid:diacylglycerol acyltransferases (PDATs) .................................29

1.2.4 Biotechnological engineering of plants using acyltransferases ........................ 30

1.3 Very long-chain polyunsaturated fatty acids (VLCPUFAs) .......................... 31

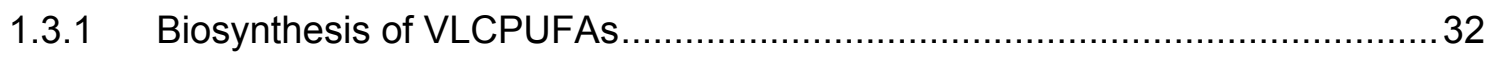

1.3.2 Enzymatic activities during VLCPUFA biosynthesis ................................ 34

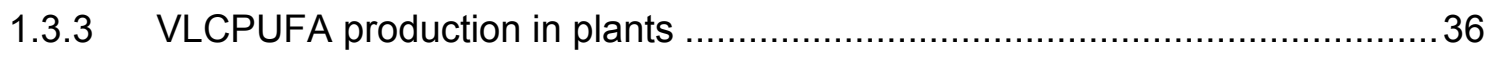

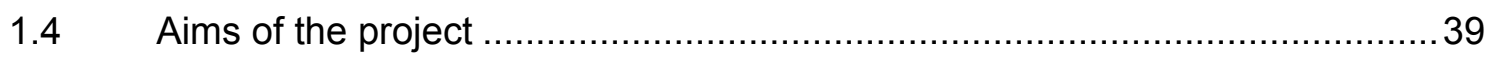

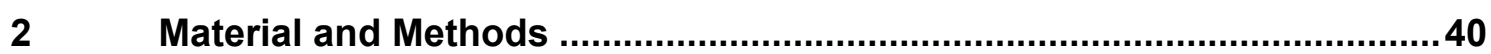

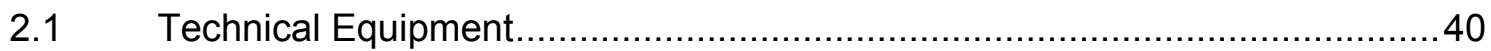

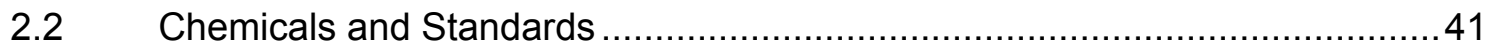

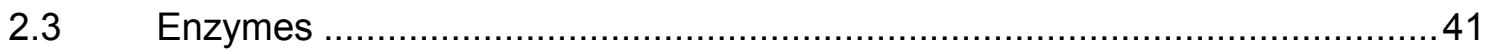

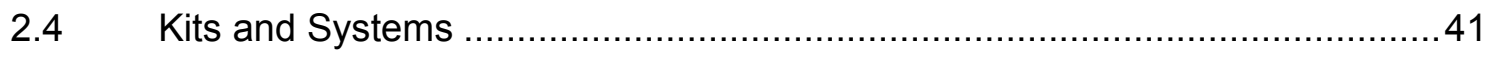

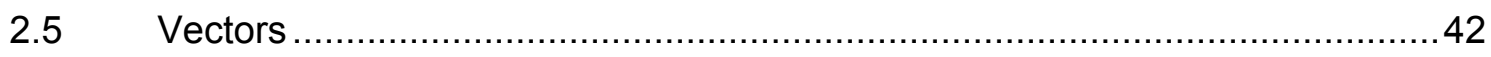

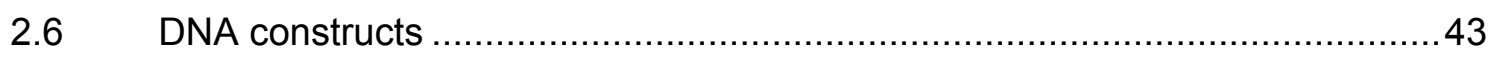

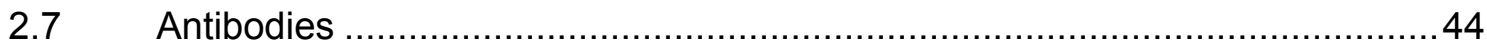




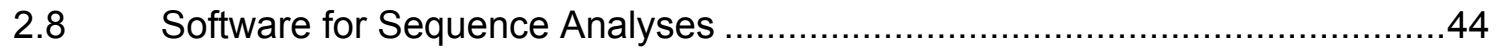

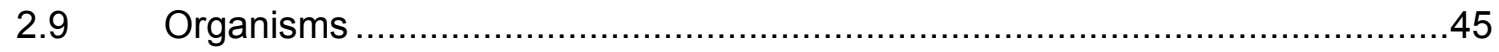

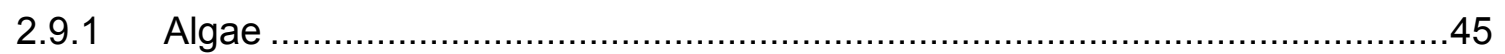

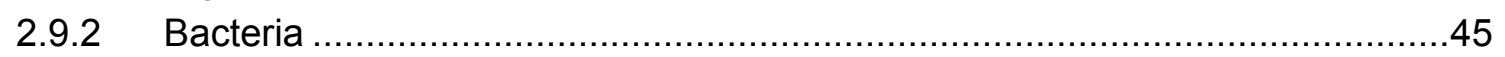

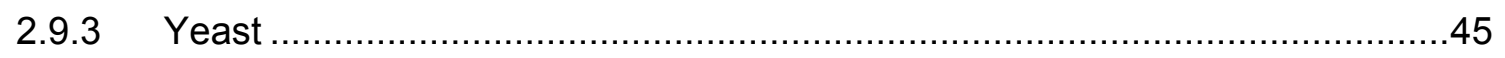

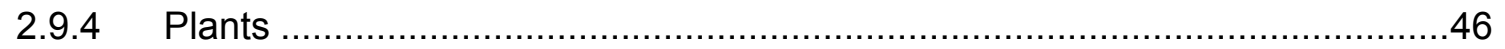

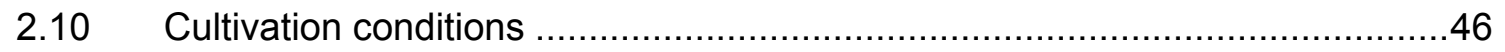

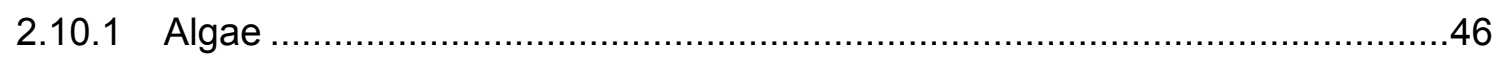

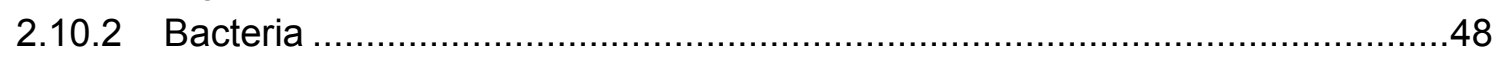

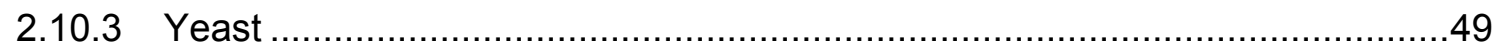

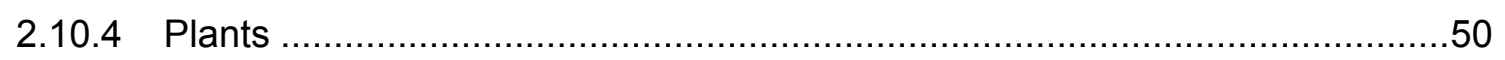

2.11 Molecular cloning methods....................................................................

2.11.1 Isolation of RNA from algae cultures or plant material ..............................51

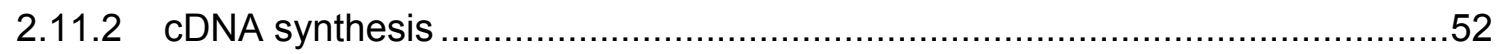

2.11.3 Isolation of genomic DNA from yeast pellets and plant material ......................52

2.11.4 Separation of DNA and RNA in agarose gels ......................................... 53

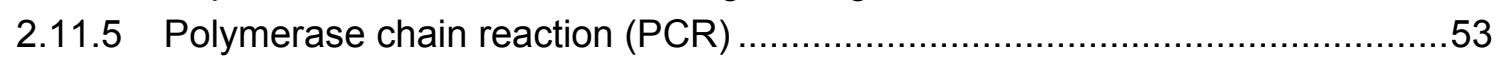

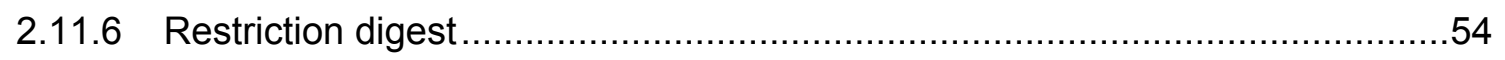

2.11.7 Isolation of DNA fragments from agarose gels ...........................................5

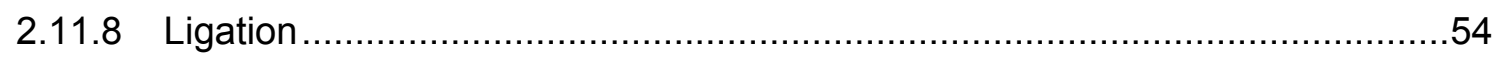

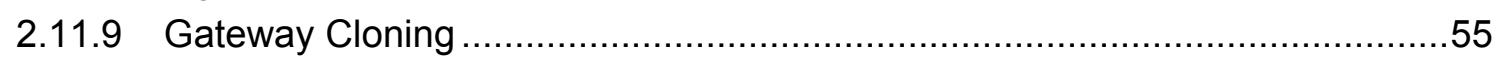

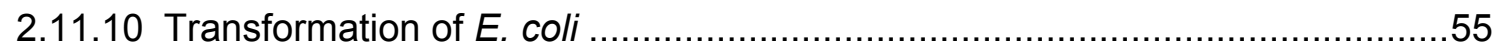

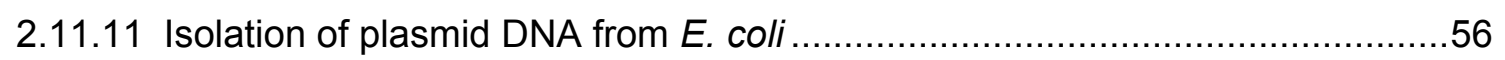

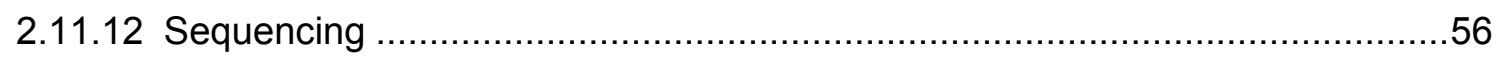

2.12 Isolation and cloning of studied cDNA sequences .........................................56

2.12.1 Cloning of microalgal acyltransferase sequences into yeast expression

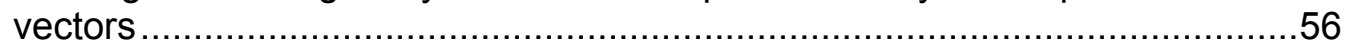

2.12.2 Isolation, codon-optimization and cloning of Old4 from O. Iucimarinus into different yeast expression vectors .......................................................... 57

2.12.3 Cloning of Egd4 from E. gracilis into different yeast expression vectors ..........58

2.12.4 Isolation and cloning of TpLACS from $T$. pseudonana into a yeast expression vector

2.12.5 Isolation and cloning of CsMGDGS from Cucumis sativus into a yeast expression vector .............................................................................. 59

2.12.6 Cloning of microalgal and plant acyltransferase sequences into plant

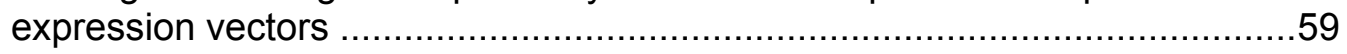

2.12.7 Cloning of Msd6 and OILPCAT into Ptd6-PSE1-Ptd5-pCAMBIA3300 ............60

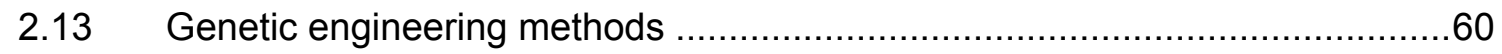

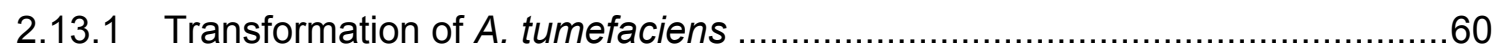

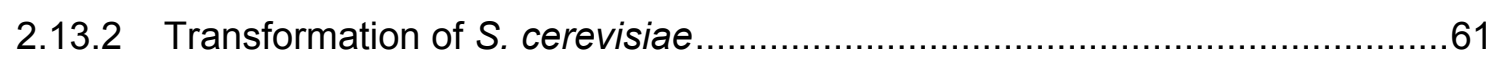

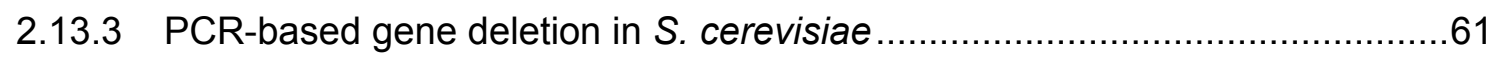

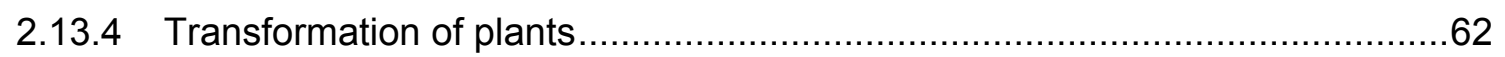

2.14 Heterologous expression of cDNAs in S. cerevisiae ....................................63

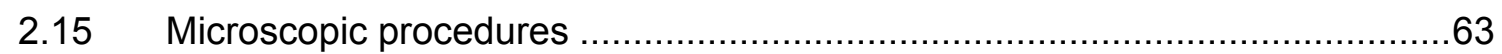

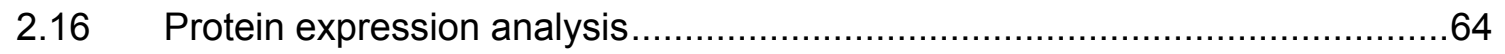




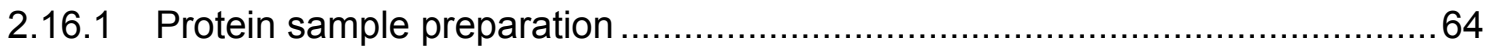

2.16.2 SDS-Polyacrylamide Gel Electrophoresis (PAGE) ......................................64

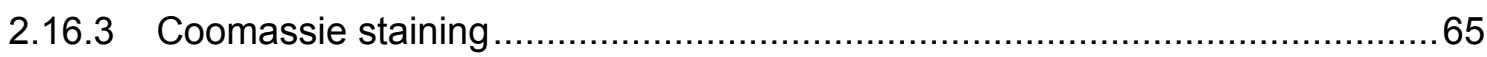

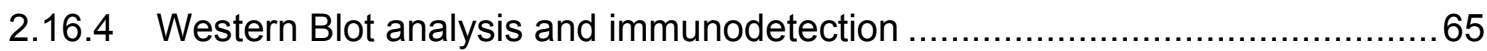

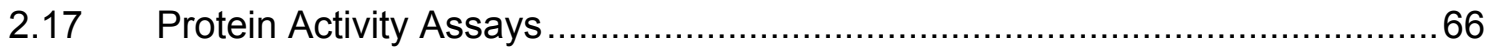

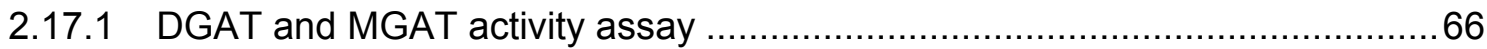

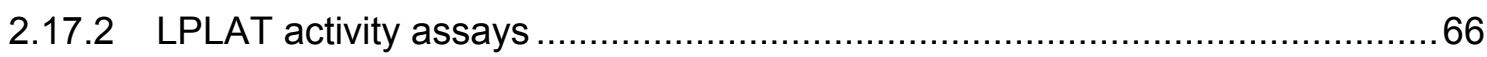

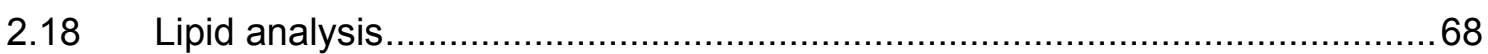

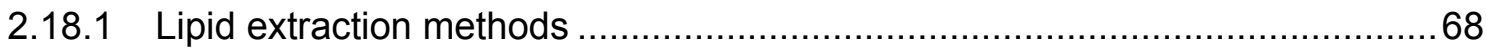

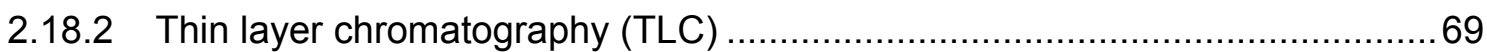

2.18.3 Isolation and derivatization of fatty acids ............................................. 70

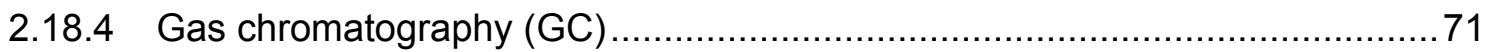

2.18.5 Extraction, derivatization and analysis of acyl-CoAs................................... 71

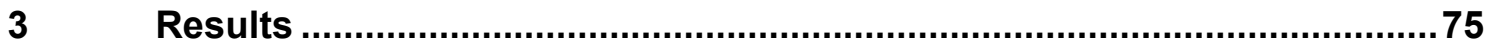

3.1 Characterization of microalgal acyltransferases in yeast .............................75

3.1.1 Characterization of the putative acyl-CoA:lysophosphatidylcholine acyltransferases OILPCATp and OtLPCATp ........................................... 75

3.1.2 In vitro studies of the acyl-CoA:diacylglycerol acyltransferase OtDGAT2Bp .. 79

3.1.3 In vivo and in vitro studies of the putative acyl-CoA:diacylglycerol acyltransferases OtDGAT2Ap and OtDGAT2Cp ................................... 81

3.1.4 Co-expression studies of different microalgal acyltransferase sequences ..... 85

3.2 Characterization of the microalgal desaturase Old4p in yeast ........................90

3.2.1 Investigation of the potential gene donor 0 . Iucimarinus ................................90

3.2.2 Identification of a putative front-end desaturase with $\Delta 4$-specificity ............... 91

3.2.3 Catalytic activity and substrate specificities of the desaturase Old4p .............99

3.2.4 Distribution of Old4p and Egd4p desaturation products in different yeast

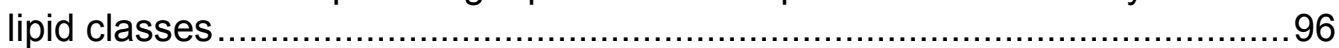

3.2.5 Comparative expression of Old4 and Egd4 and localization tests .................99

3.3 Characterization of acyltransferases, desaturases and elongases in plants 101

3.3.1 Expression of microalgal acyltransferase sequences in $A$. thaliana ............. 101

3.3.2 Complementation experiments in the $A$. thaliana tag 1-1 mutant ................... 106

3.3.3 Combination of different desaturases with an elongase in $A$. thaliana.......... 108

3.3.4 Combination of acyl-CoA- and lipid-dependent desaturases in $A$. thaliana .. 111

3.3.5 Comparison of different host plants for VLCPUFA biosynthesis ................... 113

3.3.6 Combination of microalgal acyltransferases with desaturases and an elongase in $A$. thaliana ....................................................................... 117

3.3.7 Combination of microalgal acyltransferases with desaturases and an elongase in the $A$. thaliana tag 1-1 mutant.................................................. 121

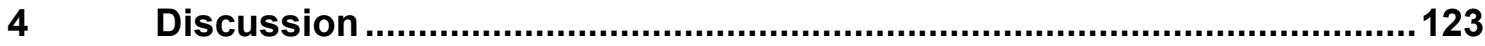

4.1 Characterization of microalgal acyltransferases in yeast .............................. 123

4.1.1 OILPCATp and OtLPCATp do not reveal LPLAT activity in vitro ..................123

4.1.2 OtDGAT2Bp prefers 18:1(n-9)-CoA as substrate in vitro .............................. 124

4.1.3 OtDGAT2Ap features a faint TAG formation ability at low expression temperatures. 
4.1.4 Co-expression of microalgal acyltransferase sequences does not change the enzymatic activity of the encoded proteins

4.2 Characterization of the microalgal desaturase Old4p in yeast....

4.2.1 The genome of $O$. lucimarinus contains a gene coding for a front-end desaturase.

4.2.2 Old4p has a preference for VLCPUFAs and desaturates (n-3)- as well as (n-6)- substrates with $\Delta 4$-specificity

4.2.3 Old4p acts in a lipid-dependent manner.

4.2.4 Old4p accumulates to solid levels in yeast cells and localizes to their endoplasmic reticulum

4.3 Characterization of acyltransferases, desaturases and elongases in plants. 130

4.3.1 OILPCATp increases levels of polyunsaturated fatty acids in the TAG fraction of Arabidopsis seeds

4.3.2 The Arabidopsis tag1-1 mutant cannot be rescued by expression of different DGAT nucleotide sequences

4.3.3 VLCPUFA amounts in transgenic Arabidopsis seeds are not stable throughout consecutive generations

4.3.4 Combination of Msd6 $p$ with lipid-dependent desaturases, PSE1 $p$ and OILPCATp increases EPA levels in transgenic $A$. thaliana seeds

4.3.5 Camelina is a promising oilseed crop for VLCPUFA production .

4.3.6 Combination of microalgal acyltransferases and primary VLCPUFA biosynthesis activities does not lead to higher product yields

4.4 Future perspectives

Bibliography

Appendix 


\section{Acknowledgements}

I want to thank all of those who supported me in any respect during the completion of the project.

To begin with, I would like to thank Prof. Dr. Ivo Feußner for giving me the opportunity to work on this interesting topic as well as for guidance, supervision and constant support during the project.

I thank Prof. Dr. Michael Thumm for being the second referee of my thesis and him as well as Dr. Henning Urlaub for being members of my thesis committee.

I would like to thank the Molecular Biology program committee for approval of the Georg-Christoph-Lichtenberg stipend from the state Lower Saxony, the "Verband der Chemischen Industrie (VCl)" for the "Chemiefonds-Stipendium" and the GGNB for the GGNB Bridging Fund.

I want to thank Dr. Ulf Ståhl for the ALE1-pYES2.1/TOPO construct, Dr. Roswitha Krick for the pUG36 vector and Prof. Dr. Sten Stymne for the yeast W303 H1246 mutant. Furthermore, I thank Dr. Mareike Heilmann, Dr. Martin Wagner, Dr. Ellen Hornung and Silke Schlenczek for providing me with DNA constructs and PCR templates.

Special thanks go to Dr. Mareike Heilmann for advice, motivation and support. I had an unforgettable time and lots of fun while working together with you in the lab.

Furthermore, I am grateful to...

... Prof. Dr. Ingo Heilmann and Dr. Martin Fulda for helpful discussions about the project.

... Dr. Mareike Heilmann, Prof. Dr. Ingo Heilmann, Karen Linnemannstöns, Dr. Alina Mosblech, Dr. Ellen Hornung and Dr. Cornelia Herrfurth for providing many constructive suggestions concerning the thesis.

... Veronika Behnen for our close collaboration during her diploma work and the students Helena Meier as well as Çağatay Alkan for their contributions to the project.

... Dr. Michael Scharnewski and Dr. Gabriel Mora for teaching me how to create a yeast knockout mutant.

... Prof. Dr. Michael Thumm, Dr. Roswitha Krick and Evelyn Welter for providing assistance with the microscope and helpful discussions concerning evaluation of the obtained data. 
... Susanne Mester who looked after my plants and protected them from mildew, aphids and sciarids. Her as well as Pia Meyer, Theres Riemekasten and Birgit Zeike I want to thank for help with sieving seeds.

... Gerd and Christoph Mader for extensive assistance whenever there were problems with computers or technical devices.

... Alina Mosblech, Bettina Gumprecht, Danuta Kaczmarzyk, Dirk Jessen, Franziska Hempel, Gabriel Mora, Jennifer Lerche, Jennifer Popko, Mareike Heilmann, Michael Scharnewski, Simone Kegel, Stephanie Werner and Veronika Behnen. Thanks for a lot of pleasurable time both in and outside the lab.

... Alina Mosblech, Cornelia Herrfurth, Ellen Hornung, Pia Meyer, Sabine Freitag and many others for lots of enjoyable lunch breaks.

... all my former and current colleagues for their helpfulness and the pleasant working atmosphere in our department.

... Dr. Steffen Burkhardt, Ivana Bacakova, Kerstin Grüniger and the GGNB team for organizing many interesting courses and events in the framework of our graduate school.

Last but not least, I would like to express my gratitude to Philipp, my family and my friends for their encouragement and constant support throughout my studies and everyday life. 


\section{Abstract}

Very long-chain polyunsaturated fatty acids (VLCPUFAs) like eicosapentaenoic acid (EPA) or docosahexaenoic acid (DHA) are health-beneficial components in the human diet. However, due to overfishing and pollution of the sea, oily sea fish as the main dietary source for these important fatty acids is decreasingly available. A promising alternative to this shortfall is the production of VLCPUFAs in transgenic oilseed crops. Although this goal has already been reached to some extent, there are still challenges which need to be met. These are the specific accumulation of VLCPUFAs in the neutral lipid fraction of seeds as well as absolute product yields, especially in case of DHA. Those aspects of heterologous VLCPUFA production might be improved by transfer of specific acyltransferases and superior desaturases from suitable VLCPUFA-producing organisms into seed lipid metabolism. Therefore, nucleotide sequences from two Ostreococcus species potentially encoding acyltransferases or a $\Delta 4$-desaturase, respectively, were characterized by expression in yeast. In these studies, none of the analyzed putative acyltransferases revealed specificity for VLCPUFAs. In contrast, the front-end desaturase Old4p from Ostreococcus lucimarinus was found to prefer VLCPUFAs to shorter-chain fatty acids and to desaturate both (n-3)- and (n-6)substrates bound to lipids. In addition to the yeast experiments, acyltransferases were also introduced individually and in combination with VLCPUFA-producing enzymes into Arabidopsis thaliana. Upon separate expression, a putative acyl-coenzyme A:lysophosphatidylcholine acyltransferase (LPCAT) sequence from O. Iucimarinus produced the strongest effects by enhancing levels of polyunsaturated fatty acids at the expense of monounsaturated fatty acids in total seed lipids. However, the combination of acyltransferases with VLCPUFA-producing enzymes did not lead to higher yields in transgenic seeds. Besides the expression studies performed with the microalgal acyltransferase sequences, also endogenous acyltransferase activities of the two different host plants $A$. thaliana and Camelina sativa were investigated by using both species for VLCPUFA-production and comparing product yields. 


\section{List of Figures}

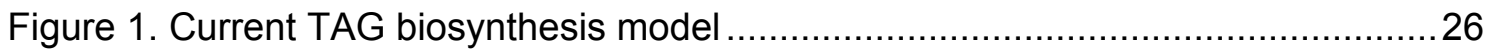

Figure 2. Molecular structures of important VLCPUFAs and their precursors .............. 32

Figure 3. VLCPUFA biosynthesis pathways ........................................................ 33

Figure 4. Protein amounts after over-expression of OtLPCAT-V5 and OILPCAT-V5 ... 76

Figure 5. OtLPCATp and OILPCATp activity in vitro ................................................ 78

Figure 6. OtDGAT2Bp activity in vitro ............................................................... 81

Figure 7. Complementation tests with the BY4741 dga1 $\Delta$ Iro1 $\Delta$ mutant by expression of OtDGAT2A, OtDGAT2B or OtDGAT2C ..........................82

Figure 8 . WS test in yeast strain $\mathrm{H} 1246$ by co-expression of mouse FAR1 with OtDGAT2A, OtDGAT2C or mouse WS ............................................. 83

Figure 9. OtDGAT2Ap and OtDGAT2Cp activity in vitro ....................................... 84

Figure 10. Acyl-CoAs after co-expression of microalgal acyltransferases in BY4741 Iro1 $\Delta$ dga $1 \Delta$ ale $1 \Delta$.

Figure 11. TAG after co-expression of microalgal acyltransferases in BY4741 Iro1 $\Delta$ dga $1 \Delta$ ale $1 \Delta$

Figure 12. PC after co-expression of microalgal acyltransferases in BY4741 Iro1

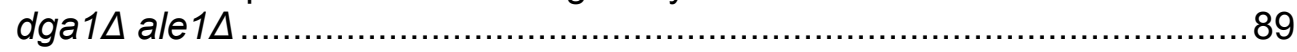

Figure 13. Fatty acid composition of total lipid extracts from O. Iucimarinus .................91

Figure 14. Phylogram of Old4p and selected functionally characterized $\Delta 4-, \Delta 5$ and $\Delta 8$-desaturases.

Figure 15. Partial alignment of the deduced amino acid sequence for Old4p with

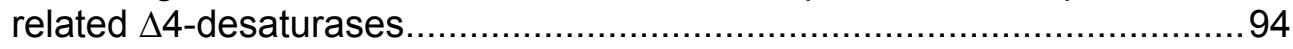

Figure 16. Gas chromatograms after expression of Old4 and Egd4 in yeast ............... 95

Figure 17. Conversion rates of supplied fatty acids by Old4p and Egd4p in yeast .......96

Figure 18. Lipid class distribution of DHA after action of Old4p or Egd4p in yeast.......97

Figure 19. Conversion rates of 22:5(n-3) after co-expression of Old4 with TpLACS

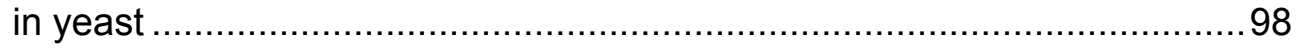

Figure 20. Results after expression of MGDGS and Old4 in yeast............................. 99

Figure 21. Protein amounts after moderate and strong expression of GFP-Old4

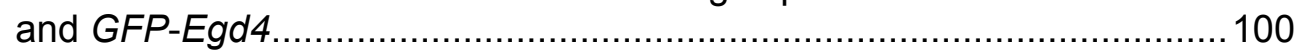

Figure 22. Localization of GFP-Old4p in yeast cells .............................................. 100

Figure 23. TAG content after expression of microalgal acyltransferase sequences in Arabidopsis.

Figure 24. Fatty acid composition after expression of OtDGAT2B and OtPDAT in Arabidopsis seeds

Figure 25. Fatty acid composition after expression of OILPCAT and OtLPCAT in Arabidopsis seeds

Figure 26. PC and TAG fatty acid compositions after expression of OILPCAT in Arabidopsis.

Figure 27. TAG content and fatty acid composition of Arabidopsis wt and tag1-1 seeds

Figure 28. TAG content after expression of different DGAT sequences in Arabidopsis wild type and tag1-1 mutant seeds . 
Figure 29. Fatty acid composition after expression of different DGAT sequences in

Arabidopsis wild type and tag1-1 mutant seeds

Figure 30. (n-3)-PUFA amounts in Arabidopsis seeds after expression of different VLCPUFA constructs.

Figure 31. Fatty acid composition of $A$. thaliana and $C$. sativa wild type seeds

Figure 32. (n-6)- and (n-3)-PUFA contents of transgenic $A$. thaliana and C. sativa T2 seeds

Figure 33. (n-6)- and (n-3)-PUFA contents of transgenic A. thaliana and C. sativa T3 seeds....

Figure 34. TAG content after co-expression of microalgal acyltransferase sequences with Pt3 in Arabidopsis

Figure 35. (n-6)- and (n-3)-PUFA contents of transgenic $A$. thaliana seeds coexpressing Pt3 with OtDGAT2B, OILPCAT or OtPDAT

Figure 36. (n-6)- and (n-3)-PUFA contents of transgenic $A$. thaliana seeds coexpressing $\mathrm{Pt} 3$ with OtLPCAT

Figure 37. TAG content after co-expression of different acyltransferase sequences with $\mathrm{Pt} 3$ in Arabidopsis tag 1-1 seeds.

Figure 38. (n-6)- and (n-3)-PUFA contents of transgenic $A$. thaliana tag1-1 seeds co-expressing Pt3 with AtDGAT1 or OtDGAT2B

\section{List of Tables}

Table 1. Overview about different in vitro assay conditions to test for OtLPCATp

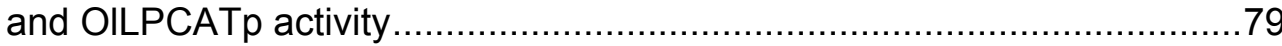

Table 2. Results from the pairwise alignment of Old4p with related proteins................92

Table 3. Overview about (n-3)- and (n-6)-PUFA amounts in Arabidopsis seeds after expression of different VLCPUFA constructs 


\section{Abbreviations}

\begin{tabular}{|c|c|}
\hline ACP & acyl carrier protein \\
\hline ad & up to \\
\hline ALA & a-linolenic acid (18:3(n-3)) \\
\hline ALE1 & $\begin{array}{l}\text { Saccharomyces cerevisiae acyltransferase for lyso- } \\
\text { phosphatidylethanolamine }\end{array}$ \\
\hline ARA & arachidonic acid $(20: 4(n-6))$ \\
\hline ATP & adenosine 5'-triphosphate \\
\hline $\mathrm{bp}$ & base pair(s) \\
\hline CDP & cytidine 5'-diphosphate \\
\hline$C L$ & cardiolipin \\
\hline CoA & coenzyme A \\
\hline СРТ & CDP-choline:diacylglycerol cholinephosphotransferase \\
\hline CsMGDGS & Cucumis sativus monogalactosyldiacylglycerol synthase \\
\hline $\mathrm{Da}$ & Dalton \\
\hline daf & days after flowering \\
\hline DAG & diacylglycerol \\
\hline dap & days after pollination \\
\hline $\mathrm{dd}_{2} \mathrm{O}$ & double distilled water \\
\hline DDT & DAG-DAG transacylase \\
\hline DGA1 & Saccharomyces cerevisiae acyl-CoA:diacylglycerol acyltransferase \\
\hline DGAT & acyl-CoA:diacylglycerol acyltransferase \\
\hline DGDG & digalactosyldiacylglycerol \\
\hline DHA & docosahexaenoic acid $(22: 6(n-3))$ \\
\hline DNA & deoxyribonucleic acid \\
\hline dNTPs & deoxyribonucleotide \\
\hline DPA & docosapentaenoic acid $(22: 5(n-3))$ \\
\hline Egd4 & Euglena gracilis $\Delta 4$-desaturase \\
\hline EPA & eicosapentaenoic acid $(20: 5(n-3))$ \\
\hline ER & endoplasmic reticulum \\
\hline al. & et alii, et aliae; and others \\
\hline$A D$ & fatty acid desaturase \\
\hline
\end{tabular}




\begin{tabular}{|c|c|}
\hline FAME & fatty acid methyl ester \\
\hline FAR1 & fatty acid reductase 1 \\
\hline FAS & fatty acid synthase \\
\hline FID & flame ionization detector \\
\hline$g$ & gramm \\
\hline G3P & glycerol-3-phosphate \\
\hline GC & gas chromatography \\
\hline GPAT & glycerol-3-phosphate acyltransferase \\
\hline HPLC & high-performance liquid chromatography \\
\hline I & liter \\
\hline LA & linoleic acid $(18: 2(n-6))$ \\
\hline LPAAT & lysophosphatidic acid acyltransferase \\
\hline LPCAT & acyl-Co:lyso-phosphatidylcholine acyltransferase \\
\hline LPLAT & acyl-Co:lyso-phospholipid acyltransferase \\
\hline LRO1 & Saccharomyces cerevisiae LCAT-related protein 1 \\
\hline MAG & monoacylglycerol \\
\hline MGAT & acyl-CoA:monoacylglycerol acyltransferase \\
\hline MGDG & monogalactosyldiacylglycerol \\
\hline $\min$ & minute(s) \\
\hline Ms3 & Msd6-PSE1-Msd5-pCAMBIA3300 \\
\hline Msd5 & Mantoniella squamata $\Delta 5$-desaturase \\
\hline Msd6 & Mantoniella squamata $\Delta 6$-desaturase \\
\hline $\mathrm{OD}_{\mathrm{x}}$ & optical density at $\mathrm{x} n \mathrm{~nm}$ \\
\hline Old4 & Ostreococcus lucimarinus $\Delta 4$-desaturase \\
\hline OILPCAT & $\begin{array}{l}\text { Ostreococcus lucimarinus acyl-Co:lysophosphatidylcholine } \\
\text { acyltransferase }\end{array}$ \\
\hline Ot3 & Otd6-PSE1-Otd5-pCAMBIA3300 \\
\hline Otd5 & Ostreococcus tauri $\Delta 5$-desaturase \\
\hline Otd6 & Ostreococcus tauri $\Delta 6$-desaturase \\
\hline OtDGAT & Ostreococcus tauri acyl-CoA:diacylglycerol acyltransferase \\
\hline OtLPCAT & Ostreococcus tauri acyl-Co:lyso-phosphatidylcholine acyltransferase \\
\hline OtPDAT & Ostreococcus tauri phospholipid:diacylglycerol acyltransferase \\
\hline$p$ & protein \\
\hline PA & phosphatidic acid \\
\hline
\end{tabular}




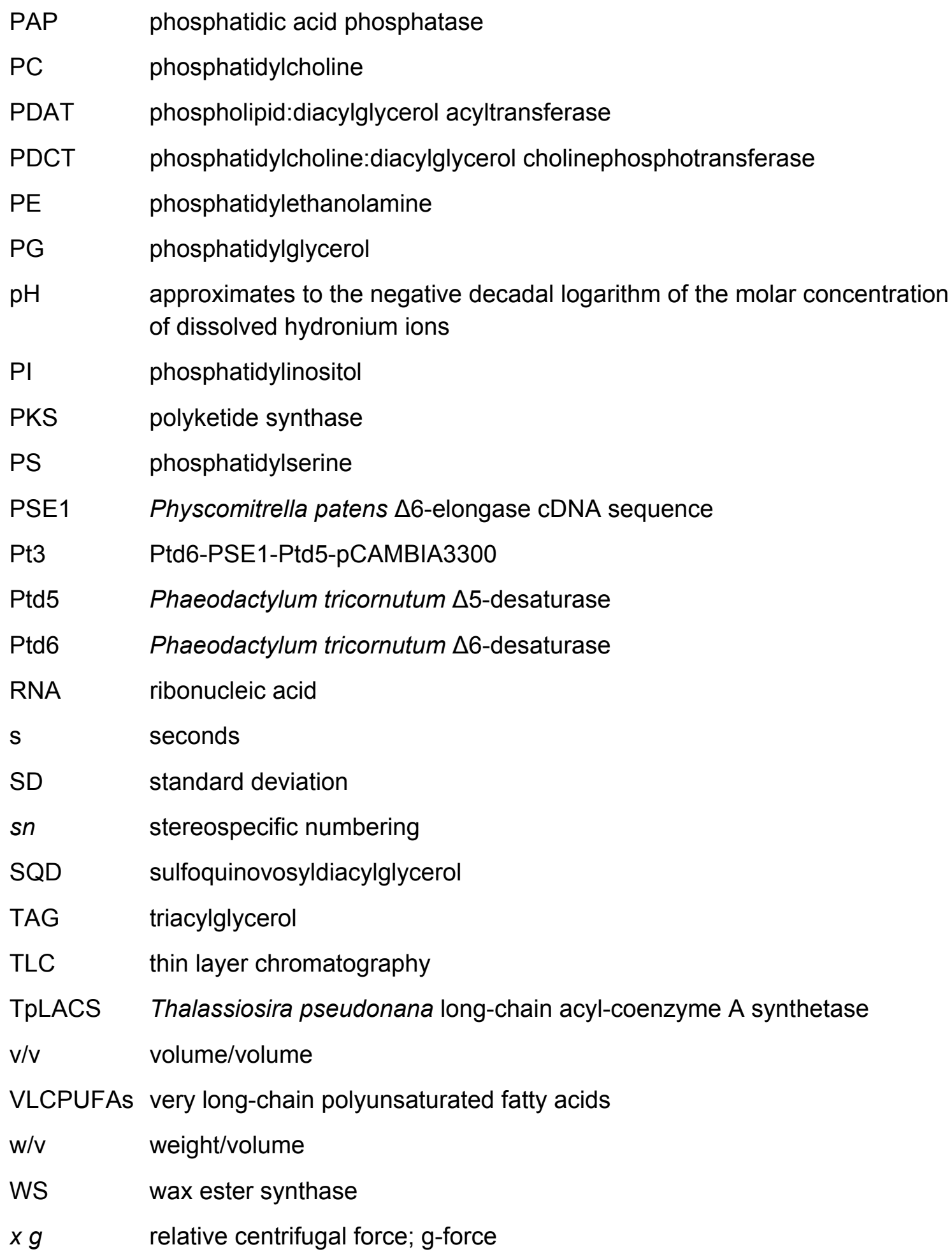




\section{Introduction}

Very long-chain polyunsaturated fatty acids (VLCPUFAs) contain 20-22 carbon atoms and have more than three double bonds. They are essential as structural components for membranes, for example in the human brain (Glomset, 2006), or as precursors for various cellular messengers (Jump, 2002). Especially due to this latter function, VLCPUFAs of the (n-3)-series are known to have health-beneficial effects like protection of the cardiovascular system (Simopoulos, 2002). VLCPUFAs are mainly produced by marine microalgae, which form the base of the aquatic food chain. Therefore, oily sea fish is rich in these fatty acids and currently constitutes the main direct nutritional source for humans, who themselves cannot produce VLCPUFAs de novo. Due to a worldwide decline of fish stocks and increasing pollution of the sea, however, oily sea fish alone is no longer able to cover the global demand for these valuable dietary components (Naylor et al., 2000; Hites et al., 2004). As a possible alternative to this shortfall, transgenic annual oilseed plants producing VLCPUFAs in their seed oil have emerged. In the past years, major advancements have been made towards VLCPUFA production in plants. Nevertheless, some hurdles still need to be overcome to reach satisfying levels of these fatty acids in oilseeds. Several different enzymatic activities for the primary biosynthesis of VLCPUFAs need to be transferred from an adequate gene donor into plants and expressed in a coordinated way in their seeds. Because the products of this primary biosynthesis should end up in the plant seed oil, they need to be incorporated into triacylglycerol (TAG) molecules. This in most of the cases cannot be successfully accomplished by the endogenous plant enzymes, which limits accumulation in planta. The distribution of VLCPUFAs within the different lipid pools of the plant cell thus is a problem, which might be solved by the transfer of acyltransferase activities from organisms producing VLCPUFAs into plants in addition to the primary biosynthetic activities.

The presented thesis deals with the characterization of different microalgal enzymatic activities considered to be able to establish VLCPUFA biosynthesis or to influence VLCPUFA distribution upon transfer into plants. The following introduction gives a brief overview about fatty acid and glycerolipid biosynthesis in plants, which are reviewed in more detail for example by (Ohlrogge and Browse, 1995; Harwood, 1996). Different types of acyltransferases and their role in the process of TAG formation will be presented. Also, the hitherto existing usage of acyltransferases in biotechnological engineering of plants will be explained. Relevance and biosynthetic pathways of VLCPUFAs as well as enzymes involved therein will be covered in the course of this introduction. And last but not least, the state of the art of VLCPUFA synthesis will be described. 


\subsection{Fatty acids and glycerolipids}

Lipids are organic molecules, which are present in every living cell. The class comprises a wide range of substances, which can be fatty acid derivatives like glycerolipids or are, like pigments and secondary compounds, synthesized via pathways independent from fatty acid metabolism. Lipids serve lots of important functions in the cell. Glycerolipids, for example, act as signalling and energy storage molecules or as major components of biological membranes. These function, due to the insolubility of lipid molecules in water, as selective barriers between different compartments and enable a directed exchange of metabolites and energy. Membranes primarily consist of lipid bilayers and therein embedded proteins, their characteristics are thus mainly influenced by the composition of the incorporated lipid species (Buchanan et al., 2000; Berg et al., 2004).

\subsubsection{The molecular structure of fatty acids}

Fatty acids are the building blocks of complex lipids. They are composed of a highly reduced hydrocarbon chain of varying length and a terminal carboxyl group. They contain an even number of carbon atoms, which can vary between eight and 32 . Fatty acids with 16 or 18 carbon atoms are most common in biological systems. Fatty acids can be either saturated or unsaturated. In the latter case, they feature one or several double bond(s) in cis-configuration between the carbon atoms at distinct positions. A prominent example for a monounsaturated fatty acid is oleic acid (18:1(n-9)) possessing 18 carbon atoms and one double bond at the ninth carbon atom proximal to the methyl end of the molecule. Another way to name this fatty acid is $18: 1^{\Delta 9}$. The underlying $\Delta$-nomenclature is indicating the distance between the double bond position and the carboxyl end of the molecule. In case of two or more double bonds in one fatty acid, double bonds are interrupted in nearly all cases by at least one methylene group. Decreasing length and increasing degree of unsaturation of a fatty acid are lowering the melting point of this fatty acid and its derivatives (Buchanan et al., 2000; Berg et al., 2004).

\subsubsection{De novo biosynthesis of fatty acids}

In plants, fatty acids are synthesized in the plastids by action of the fatty acid synthase (FAS) complex. This complex consists of several different proteins that altogether catalyze the formation of fatty acids from acetyl-coenzyme A (CoA) and malonyl-CoA. Fatty acid biosynthesis starts with the formation of malonyl-CoA by adenosine 5'triphosphate (ATP)-dependent carboxylation of acetyl-CoA, catalyzed by the enzyme acetyl-CoA carboxylase. The malonyl moiety is transferred onto acyl carrier protein (ACP), the first component of the FAS complex, and thereupon decarboxylated. In the following condensation reaction, acetyl-CoA is added and three subsequent reactions 
of reduction, dehydration and another reduction result in the formation of acyl-ACP. This acyl-ACP is then further elongated in two-carbon chain units by cyclic repetition of condensation with malonyl-CoA, two reduction steps and a dehydration reaction. The process ends as soon as 16:0-ACP or 18:0-ACP are formed (Ohlrogge and Jaworski, 1997). Some intermediates of fatty acid biosynthesis are barely detectable. From this fact it can be deduced that the interplay between the different enzymatic activities is highly efficient and some kind of substrate channeling occurs during fatty acid synthesis (Buchanan et al., 2000). 16:0-ACP and 18:0-ACP can be used as substrate for desaturation by ACP-dependent desaturases. These soluble enzymes mostly introduce double bonds at the $\Delta 9$-position. One prominent example for this type of enzyme is the stearoyl-ACP desaturase, which catalyzes the formation of oleoyl-ACP (Shanklin et al., 1991; Thompson et al., 1991). The acyl chains can also be directly transferred from ACP onto glycerolipids by distinct acyltransferase activities for the synthesis of "prokaryotic" lipids (Harwood, 1996). Alternatively, the acyl moiety of the acyl-ACP can be cleaved off by thioesterases and is exported to the cytoplasm. There it is, esterified to CoA, used as substrate for the synthesis of "eukaryotic" lipids at the endoplasmic reticulum (ER) (Roughan and Slack, 1982; Somerville and Browse, 1991). As fatty acids mostly do not exist as free molecules in the cell, but rather are bound to glycerolipids, glycerolipid composition and biosynthesis will be explained in the following sections.

\subsubsection{The composition of glycerolipids}

Glycerolipids are important for cell membrane structure as well as energy storage and take an active part in various signalling events. They consist of a glycerol backbone connected with up to three fatty acids via ester bonds. The positions of the fatty acids at the glycerol part of the molecule are given according to the stereospecific numbering (sn) nomenclature as $s n-1, s n-2$ or $s n-3$.

Glycerolipids can be subdivided into different classes. Glycolipids constitute one class and contain a sugar headgroup bound to glycerol instead of the third fatty acid. Monogalactosyldiacylglycerol (MGDG), digalactosyldiacylglycerol (DGDG) or sulfoquinovosyldiacylglycerol (SQD) are members of this group and are predominantly found in plastidial membranes. Phospholipids, as the second class, serve important functions in membranes and in cell signalling. They exhibit a polar phosphate-containing headgroup connected to glycerol. The headgroup can consist either of phosphate alone, the derived lipid is then named phosphatidic acid (PA), or of phosphate connected with alcohols like glycerol, choline, ethanolamine, inositol or serine. The corresponding lipids are named phosphatidylglycerol (PG), phosphatidylcholine (PC), phosphatidylethanolamine (PE), phosphatidylinositol (PI) or phosphatidylserine (PS). Another representative of this group of lipids is the mitochondrial lipid cardiolipin $(C L)$, which is composed of two PG molecules that are interconnected at their glycerol portion. Neutral lipids make up the third class of glycerolipids and do not feature a polar headgroup. Common members are monoacylglycerols (MAGs), diacylglycerols (DAGs) and triacylglycerols (TAGs). They contain one, two or three fatty acids, respectively, 
esterified to the glycerol backbone. DAG acts as central precursor in lipid metabolism and as signalling molecule, whereas TAG is an essential storage compound in the cell (Buchanan et al., 2000; Berg et al., 2004).

\subsubsection{Biosynthesis of glyco- and phospholipids}

Plant membrane lipids can be formed in two different pathways. The "prokaryotic" pathway (Roughan and Slack, 1982; Somerville and Browse, 1991) initially leads to the formation of PA in plastids by sequential activity of acyl-ACP:glycerol-3-phosphate acyltransferase (GPAT) and acyl-ACP:lyso-phosphatidic acid acyltransferase (LPAAT) on glycerol-3-phosphate (G3P). PA can then be further converted into cytidine 5'diphosphate (CDP)-DAG or DAG. These compounds are then used as substrates for formation of PG or galactolipids, respectively (Browse and Somerville, 1991; Ohlrogge and Browse, 1995). Due to the distinct enzyme specificities of the participating acyltransferases, lipids derived from this pathway are enriched in palmitic acid (16:0) at the sn-2 position and in most cases oleic acid at the sn-1 position. Fatty acids associated with complex lipids can be further modified by plastidial desaturation activities (Somerville and Browse, 1991).

The "eukaryotic" pathway basically employs the same acyltransferase activities and also leads at first to the formation of PA and subsequently of CDP-DAG or DAG. But in contrast to the "prokaryotic pathway", the reaction series takes place at the ER and requires acyl-CoAs as substrates. DAG is further converted to PC or PE, CDP-DAG is used for formation of PG, PS or PI. Lipids derived from this pathway are enriched in 18carbon chain fatty acids at the sn-2 position and palmitic acid at the sn-1 position (Ohlrogge and Browse, 1995). Fatty acids bound to PC derived from the "eukaryotic pathway" can be further modified by desaturases which convert oleic acid further into linoleic acid (18:2(n-6), LA) and a-linolenic acid (18:3(n-3), ALA) (Arondel et al., 1992; Okuley et al., 1994).

Both "prokaryotic" and "eukaryotic" pathways do not exist separated from each other, but they are interconnected by a common DAG pool (Browse et al., 1986). Also, lyso$\mathrm{PC}$ and PA are transported between plastid and ER and are used for lipid synthesis at both sites (Roughan and Slack, 1982; Somerville and Browse, 1991). Thus, all glycerolipids in the cell are involved in continuous exchange. Furthermore, phospholipids underlie constant turnover at their sn-2 acyl moiety in the so-called Land's cycle (Lands, 1958) by concerted action of phospholipases, acyl-CoA synthetases and lysophospholipid acyltransferases (LPLATs) (Fulda et al., 1997; Lands, 2000; Shimizu et al., 2006). 


\subsubsection{Biosynthesis of triacylglycerol}

TAG is a major storage compound in plant seeds and represents up to $60 \%$ of the seed dry weight. Studies conducted by Shockey and co-workers could show that specific ER regions are dedicated to TAG synthesis and that the required enzymes are assembling in these subdomains (Shockey et al., 2006). The traditional view of TAG formation (Figure 1) shares some common features with the biosynthesis of phospholipids via the "eukaryotic" pathway (Browse and Somerville, 1991; Ohlrogge and Browse, 1995). PA is built up at the ER from G3P by sequential acyl-CoA-dependent GPAT and LPAAT activity (Stymne, 1987). Then, PA is dephosphorylated by PA phosphatase (PAP) and thereby converted into DAG. Alternatively, DAG can also be produced from MAG by an acyl-CoA:monoacylglycerol acyltransferase (MGAT), as it could be shown for peanut (Arachis hypogaea) cotyledons by Tumaney and co-workers (Tumaney et al., 2001).

The fatty acid composition of DAG can be affected not only by the previously mentioned acyltransferases but also by various remodelling processes. DAG-DAG transacylase (DDT) is an example for direct influence because it transfers acyl chains from one DAG molecule onto another and thereby produces MAG and TAG, as it could be shown for castor bean (Ricinus communis) (Lehner and Kuksis, 1996) and safflower (Carthamus tinctorius) (Stobart et al., 1997). Also, the CDP-choline:diacylglycerol cholinephosphotransferase (CPT) can convert PC into DAG and thereby provides a mechanism for synthesis of polyunsaturated DAG species. Fatty acids in this way can first be desaturated while bound to $P C$ and are then transferred into the DAG pool (Browse and Somerville, 1991; Ohlrogge and Browse, 1995). In a similar manner, phosphatidylcholine:diacylglycerol cholinephosphotransferase (PDCT) transfers the headgroup of PC onto DAG and thus directly changes fatty acid composition in the DAG pool of the cell (Lu et al., 2009a). The remodelling events between DAG and PC currently become more and more important because recent studies could show that most of the newly synthesized fatty acids from the plastid are first transferred onto PC before they reach other glycerolipid species by acyl editing events at both sn-1 and sn-2 positions (Bates et al., 2007; Bates et al., 2009). These editing events can for example be mediated by the action of acyl-CoA:lyso-phosphatidylcholine acyltransferase (LPCAT). This enzyme transfers acyl chains from the sn-2 position of PC onto CoA and vice versa (Stymne and Stobart, 1984). Both of these pools are used as raw material for neutral lipid synthesis.

The last step of TAG biosynthesis is carried out by two different enzymatic activities (Figure 1). Acyl-CoA-dependent TAG formation is performed by acylCoA:diacylglycerol acyltransferase (DGAT) (Ohlrogge and Browse, 1995). This enzyme transfers an acyl chain from the CoA pool onto DAG and thereby produces TAG and free CoA. Phospholipid:diacylglycerol acyltransferase (PDAT), on the other hand, mediates acyl-CoA-independent TAG synthesis by transferring an acyl chain from the PC 
pool onto DAG and in turn releasing TAG and lyso-PC (Dahlqvist et al., 2000). Upon formation at the ER, TAG is then accumulating in phospholipid monolayer-enclosed compartments named oil bodies (Schwarzenbach, 1971; Stobart et al., 1986; Murphy and Vance, 1999). These oil bodies are approximately $1 \mu \mathrm{m}$ in diameter and contain a number of important structural proteins, for example oleosins, at their surface (Kim et al., 2002). They are the storage rooms for newly synthesized TAGs until they are accessed by lipases upon reserve remobilisation.

The seed oils of some plant species are enriched in unusual fatty acids that have special characteristics like particular functional groups. These fatty acids are normally not found in the membrane lipids of the respective species (Millar et al., 2000). Castor bean oil, for example, is rich in ricinoleic acid (12-hydroxy-18:1(n-9)) which accounts for up to $90 \%$ of total fatty acids in the seed oil. It is produced at the ER while bound to the sn-2 position of PC and then transported by highly efficient acyltransferase activities into the TAG fraction (Kroon et al., 2006).

Due to the particular importance of acyltransferases in the process of glycerolipid biosynthesis, the following section is dedicated to the presentation of some important representatives of this group of enzymes. 


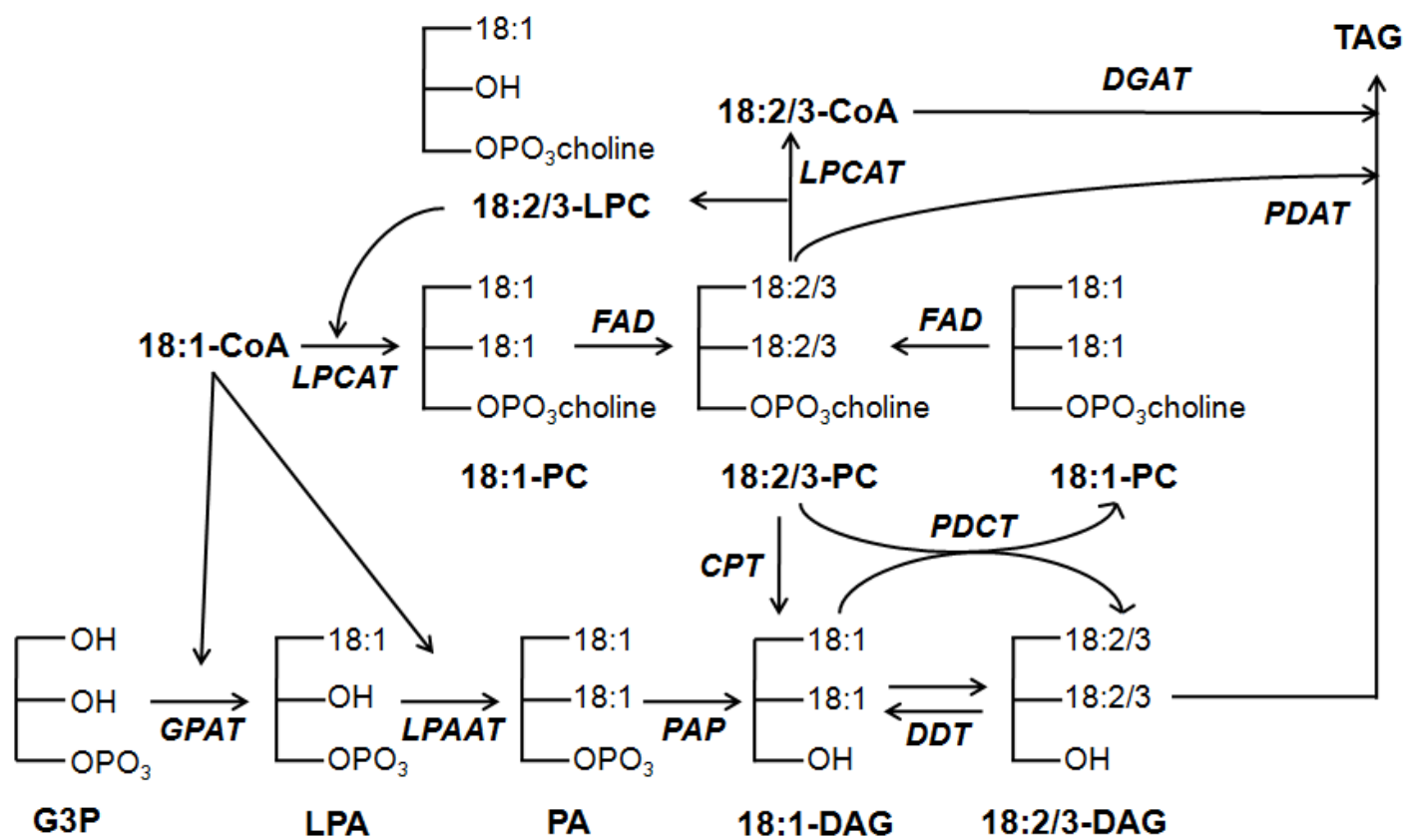

\section{Figure 1. Current TAG biosynthesis model}

Abbreviations, in alphabetical order: CoA, Coenzyme A; CPT, CDP-choline:diacylglycerol cholinephosphotransferase; DAG, diacylglycerol; DDT, diacylglycerol:diacylglycerol acyltransferase; DGAT, acyl-CoA:diacylglycerol acyltransferase; FAD, fatty acid desaturase; G3P, Glycerol-3Phosphate; GPAT, acyl-CoA:glycerol-3-phosphate acyltransferase; LPA, lyso-phosphatidic acid; LPAAT, acyl-CoA:lyso-phosphatidic acid acyltransferase; LPC, lysophosphatidylcholine; LPCAT, acyl-CoA:lyso-phosphatidylcholine acyltransferase; PA, phosphatidic acid; PAP, phosphatidic acid phosphatase; PC, phosphatidylcholine; PDAT, phospholipid:diacylglycerol acyltransferase; PDCT, phosphatidylcholine:diacylglycerol cholinephosphotransferase; TAG, triacylglycerol. Not depicted is the synthesis of DAG from monoacylglycerol (MAG) via acylCoA:monoacylglycerol acyltransferase (MGAT). Explanations are given in the text (section 1.1.5). Figure extended on the basis of (Lu et al., 2009a). 


\subsection{Acyltransferases}

\subsubsection{Acyl-CoA:lyso-phosphatidylcholine acyltransferases (LPCATs)}

LPCATs are important players in the process of acyl editing by mediating the transfer of acyl chains between the sn-2 position of PC and CoA pool (Figure 1). Stymne and Stobart identified LPCAT activity in microsomal preparations of rat lung and safflower cotyledons already in the 1980s (Stymne and Stobart, 1984, 1985). However, the first cloning and biochemical characterization of a cDNA encoding a LPCAT from mouse was published only recently (Chen et al., 2006; Nakanishi et al., 2006). These studies showed that LPCAT activity is essential for the synthesis of di-16:0-PC for lung surfactant. In 2007, several studies documented that the yeast gene YOR175C, also known as acyltransferase for lyso-PE (ALE1), is encoding an acyl-CoA-dependent LPLAT protein capable of acylation of lyso-PC but also of other substrates like lyso-PA, lyso-PE, lyso-PG, lyso-PS and lyso-PI (Benghezal et al., 2007; Chen et al., 2007; Jain et al., 2007; Riekhof et al., 2007; Tamaki et al., 2007). The enzyme was shown to accept substrates with two to 20 carbon atoms and preferred unsaturated acyl chains with 16 to 20 carbon atoms (Tamaki et al., 2007). It was found to be highly enriched in the mitochondria-associated ER membrane (Riekhof et al., 2007) and was classified as member of the membrane-bound O-acyltransferase superfamily (Hofmann, 2000). This superfamily contains several acyltransferases with limited sequence similarity in their putative active site region (Stahl et al., 2008). It exhibits two characteristic amino acids, the first being either histidine or asparagine, the second always being histidine, which is located in the center of a longer hydrophobic stretch. Both amino acids are possibly essential for the catalytic activity (Hofmann, 2000). The first plant LPCATs were identified in 2008 by Ståhl and co-workers in Arabidopsis. Biochemical analyses revealed broad specificity in LPLAT activities for both enzymes, encoded by At1g12640 and At1g63050 (Stahl et al., 2008).

Recently, Dr. M. Wagner identified nucleotide sequences for putative LPCATs in the microalgae Ostreococcus tauri and Ostreococcus lucimarinus. The enzyme from the latter-mentioned species was found to partially complement a yeast mutant deficient in endogenous LPCAT activity (Wagner, 2008).

\subsubsection{Acyl-CoA:diacylglycerol acyltransferases (DGATs)}

DGATs are an essential part of the conventional TAG formation pathway by mediating the transfer of acyl chains from the CoA pool onto the sn-3-position of DAG (Figure 1). In Arabidopsis, the tag1-1 (AS11) mutant derived from an ethyl methanesulfonate mutagenesis screen was identified in 1995. Mutant seeds revealed reduced levels of 18:1 and 20:1 and a concomitant accumulation of ALA. Furthermore, overall seed TAG content was reduced and seed development was delayed. The authors assumed a 
reduced DGAT activity, which leads to higher substrate levels for desaturation (Katavic et al., 1995). In subsequent studies, the corresponding cDNA sequence encoded by the gene At2g19450 and named TAG1, was isolated, expressed in yeast or insect cells and biochemically analyzed by different groups (Hobbs et al., 1999; Routaboul et al., 1999; Zou et al., 1999). Since then, several DGAT1 enzymes have been identified in various plants like tobacco (Nicotiana tabacum), rapeseed (Brassica napus), burning bush (Euonymus alatus), castor bean, soybean (Glycine max), tung tree (Vernicia fordii), nasturtium (Tropaeolum majus) and Echium pitardii (Bouvier-Nave et al., 2000; Nykiforuk et al., 2002; He et al., 2004; Milcamps et al., 2005; Shockey et al., 2006; Wang et al., 2006; Xu et al., 2008; Mañas-Fernández et al., 2009).

In 2001, Lardizabal and co-workers identified two cDNAs encoding putative DGATs from the oleaginous fungus Mortierella ramanniana, expressed them in insect cells and assayed cell membranes for DGAT activity. With these experiments they could verify DGAT activity of the encoded proteins. Their sequences, however, were different from the previously described DGATs and thus were classified as DGAT2 enzymes. Homologues of these enzymes were found to be present in fungi, plants and mammals (Lardizabal et al., 2001). In Saccharomyces cerevisiae, the gene YOR245c, also named DGA1, also encodes a DGAT2-like protein. This was found to be the major player for TAG formation in yeast (Sorger and Daum, 2002). Recently, a DGAT2 protein was also identified in castor bean and its encoding gene was shown to be stronger expressed in seeds than the $R$. communis DGAT1 gene. The authors thus concluded an important role of DGAT2 for seed TAG synthesis (Kroon et al., 2006). Shockey and co-workers achieved similar results in tung tree when they compared expression of DGAT1 and DGAT2 genes in this organism. Furthermore, they found out that both enzymes produced different TAG species and were located in distinct ER regions which were not overlapping (Shockey et al., 2006).

Whereas DGAT1 and DGAT2 enzymes are membrane-bound proteins, a third independent cytosolic DGAT enzyme, designated as DGAT3, could be identified in developing peanut cotyledons by Saha and co-workers (Saha et al., 2006). This soluble protein shares more sequence similarities with bacterial bifunctional wax ester synthase (WS)/DGAT enzymes than with DGAT1 and DGAT2 proteins. These can use both fatty alcohols and DAG as acyl acceptor. The presence of bifunctional WS/DGAT proteins has been reported for the bacterium Acinetobacter (Kalscheuer and Steinbüchel, 2003; Stoveken et al., 2005) as well as for plants (King et al., 2007; Li et al., 2007).

Very recently, the first microalgal DGATs were identified from $O$. tauri and characterized by Dr. M. Wagner. The sequences were named OtDGAT2A and OtDGAT2B according to their structural similarity to other DGAT2 sequences. The protein encoded by OtDGAT2B revealed much higher activity in yeast and accepted saturated as well as mono- and polyunsaturated acyl-CoAs as substrates (Wagner, 2008). 


\subsubsection{Phospholipid:diacylglycerol acyltransferases (PDATs)}

PDATs mediate TAG formation by transfer of acyl chains from the sn-2 position of phospholipids like PE and PC onto the sn-3 position of DAG (Figure 1). Acyl-CoAindependent TAG synthesis in plants was observed for the first time in microsomal preparations from different oil seeds (Stobart et al., 1997; Dahlqvist et al., 1998). The responsible enzymatic activity was initially discovered a few years later in $S$. cerevisiae (Dahlqvist et al., 2000; Oelkers et al., 2000). The yeast gene YNR008w was demonstrated to encode a PDAT protein structurally similar to mammalian lecithin-cholesterol acyltransferases. Therefore, the gene was designated as LCAT-related protein 1 (LRO1). The activity of the respective PDAT enzyme depended on the headgroup of the phospholipid acyl donor, the transferred acyl chain and the acyl chains of the acyl acceptor DAG (Dahlqvist et al., 2000). Complete knock-out of the gene led to a strong decrease in TAG content in yeast, whereas over-expression led to an increase of PDAT activity in microsomal assays (Oelkers et al., 2000). Dahlqvist and co-workers also performed in vitro studies with microsomal preparations from sunflower (Helianthus annuus), castor bean and Crepis palaestina. These showed different substrate preferences for the transferred acyl chains which also reflected the TAG composition of the respective plant species (Dahlqvist et al., 2000). In 2004, the first plant PDAT protein, encoded by the gene At5g13640, was identified in Arabidopsis. Its enzymatic activity was verified by over-expression of the corresponding cDNA in planta and subsequent in vitro tests with microsomal preparations from different plant tissues. AtPDAT1p was shown to use different phospholipids as acyl donors and had a threefold preference for the $s n-2$ position over the $s n-1$ position of phospholipids. Acyl groups containing ten to 22 carbon atoms were transferred but, surprisingly, the highest activity was found for acyl chains containing double bonds, epoxy or hydroxyl groups (Stahl et al., 2004). In a complementary approach, Mhaske and co-workers characterized an Arabidopsis knock-out line for At5g13640 and investigated fatty acid content and composition of seeds in comparison to wild type seeds. They could not find any differences and thus concluded that AtPDAT1p does not play an important role for TAG synthesis in seeds (Mhaske et al., 2005). This idea, however, was disproven by a recent study from Zhang and co-workers. They showed that silencing of AtPDAT1 via RNAi in the tag1-1 background or vice versa silencing AtDGAT1 in the atpdat1 background led to a 70-80 \% decrease in seed oil, furthermore to pollen sterility and disruption of embryo development. From these data, the authors concluded an overlapping effect of AtDGAT1p and AtPDAT1p in seed oil TAG synthesis (Zhang et al., 2009).

In 2008, a microalgal putative PDAT from O. tauri was identified by Dr. M. Wagner. The encoded protein was shown to complement a yeast mutant deficient in endogenous TAG formation upon addition of polyunsaturated fatty acids of the (n-3)- or the (n-6)series with 18 or 20 carbon atoms (Wagner, 2008). 


\subsubsection{Biotechnological engineering of plants using acyltransferases}

The first reports on the over-expression of acyltransferase sequences in seeds in order to change seed lipid metabolism of plants were published in the mid-90s. In these studies, sequences coding for LPAATs from two meadowfoam species (Limnanthes alba alba and Limnanthes douglasii) were expressed in rapeseed. This resulted in altered proportions of $s n-2$ erucic acid in seed oil of the host plant and thus influenced stereochemical composition of the TAG fraction (Lassner et al., 1995; Brough et al., 1996). Later expression experiments with a yeast LPAAT sequence in planta not only changed the composition of TAGs, but also the total seed oil content. The authors of this study constitutively expressed the sequence for the yeast LPAAT sequence SLC1 in Arabidopsis and rapeseed, which resulted in an 8-48 \% increase in seed oil content. Furthermore, proportions and amounts of very long-chain fatty acids in the TAG fraction, especially at the sn-2 position of TAG, were strongly enhanced (Zou et al., 1997).

However, most reports in the past have dealt with the implementation of DGAT activities into Arabidopsis or oilseed crops. Jako and co-workers in 2001 were the first ones, who over-expressed the sequence encoding AtDGAT1p in Arabidopsis seeds. In correlation with the measured expression levels, this positively influenced oil deposition and average seed weight (Jako et al., 2001). In subsequent studies using a similar approach, increases in the oil content could be achieved in soybean, maize (Zea mays) and rapeseed by over-expression of either foreign or endogenous DGAT sequences (Lardizabal et al., 2008; Weselake et al., 2008; Zheng et al., 2008; Taylor et al., 2009). Recently, also the production of the uncommon fatty acid ricinoleic acid in the host plant Arabidopsis could be enhanced by co-expression of castor bean sequences encoding fatty acid hydroxylase and DGAT activity from 17-30 \% compared to the expression of the fatty acid hydroxylase sequence alone (Burgal et al., 2008).

Up to now, there is only one study, which reports on the implementation of a LPLAT activity into the seed metabolism of a host plant. Wu and co-workers established VLCPUFA production in Indian mustard (Brassica juncea) and in this context also introduced a putative LPLAT sequence from Thraustochytrium sp. into plants. Thraustochytrium sp. is rich in docosahexaenoic acid (DHA, 22:6(n-3)) (Lewis et al., 1999). The enzyme was therefore believed to have a preference for VLCPUFAs and might enhance product yields in the host plant. However, it is not clear whether expression of the putative acyltransferase sequence had any effect (Wu et al., 2005). 


\subsection{Very long-chain polyunsaturated fatty acids (VLCPUFAs)}

Important representatives of this group of fatty acids are the (n-6)-fatty acid arachidonic acid (20:4(n-6), ARA) as well as the (n-3)-fatty acids eicosapentaenoic acid (20:5(n-3), EPA) and DHA. All of them are depicted with their respective precursors in Figure 2. The nomenclature of the fatty acids is derived from the position of the first double bond proximal to the methyl end of the molecule. Is this double bond at the sixth position, the respective fatty is ranked among the (n-6)-group. In case the double bond is at the third position, the fatty acid is part of the (n-3)-series. The alternative $\Delta$-nomenclature in Figure 2 indicates the double bond positions counted from the carboxyl end of the molecule.

VLCPUFAs are important membrane constituents and essential for example during fetal development as well as for proper retina and brain function (Carlson et al., 1993; Crawford et al., 1997; Giusto et al., 2000). Furthermore, VLCPUFAs serve important functions as cellular messengers. ARA is the precursor for thromboxanes, leukotrienes and prostaglandins which are activating inflammation processes. EPA and DHA can be converted for example into resolvins, which have anti-inflammatory effects (Hwang, 2000; Funk, 2001; Jump, 2002). The derivatives of (n-3)-VLCPUFAs are known to have health-beneficial effects like protection of the cardiovascular system or reduction of inflammatory processes when taken up in sufficient amounts (Demaison and Moreau, 2002; Simopoulos, 2002; Calder, 2004).

Humans, like all mammals, lack $\Delta 12$ - and $\Delta 15$-desaturases to convert oleic acid into LA and ALA, respectively (Nakamura and Nara, 2003). Therefore, they cannot synthesize these fatty acids de novo, but have to take them up with their nutrition as essential precursors for VLCPUFAs (Goyens et al., 2006). In addition, also the direct dietary supply with VLCPUFAs is crucial because conversion rates from LA and ALA into their respective very long-chain derivatives is rather low in men (Gerster, 1998; Pawlosky et al., 2001; Brenna, 2002; Goyens et al., 2006). At present, the ratio between (n-6)- and (n-3)-fatty acids in the Western diet is much too high. Thus, especially the direct uptake of (n-3)-VLCPUFAs would act towards the undersupply with these fatty acids and would lower the (n-6) over (n-3) ratio (Goyens et al., 2006; Simopoulos, 2006).

Marine microalgae are the main primary producers of VLCPUFAs and constitute the base of the aquatic food chain. That is why currently oily sea fish, like tuna, salmon or herring, is the most important dietary VLCPUFA source for humans. However, due to problems like overfishing (Naylor et al., 2000) and pollution of the sea (Hites et al., 2004), research for sustainable production of VLCPUFAs, for example in oilseed crops, is ongoing and was reviewed recently in (Venegas-Calerón et al., 2010). 
A

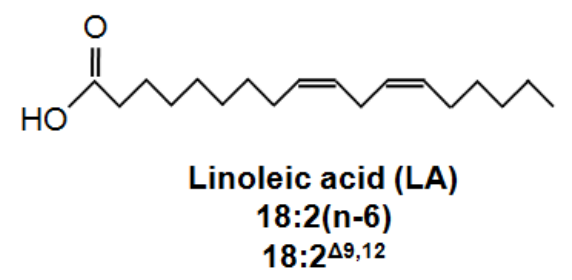

算

Arachidonic acid (ARA)

20:4(n-6)

$20: 4^{\Delta 5,8,11,14}$
B<smiles>C/C=C\C=C/C=C\CCCCCCC(=O)O</smiles><smiles>C/C=C\C=C/C/C=C/C=C/C/C=C\CCCC(=O)O</smiles>

Eicosapentaenoic acid (EPA)

20:5(n-3)

20:5 $55,8,11,14,17$

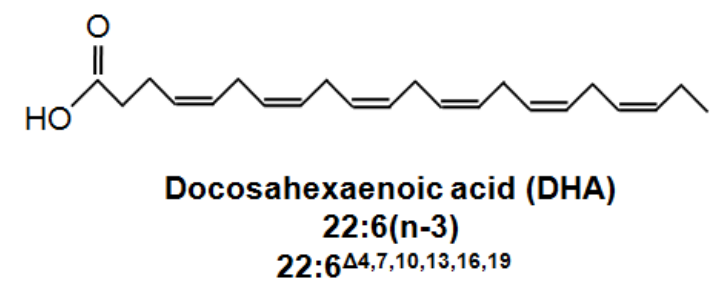

Figure 2. Molecular structures of important VLCPUFAs and their precursors (A) (n-6)-(VLC)PUFAs (B) (n-3)-(VLC)PUFAs

\subsubsection{Biosynthesis of VLCPUFAs}

Synthesis of VLCPUFAs is accomplished via two completely different systems. On the one hand, there is the oxygen-independent polyketide synthase (PKS)-like system found in marine bacteria and eukaryotes (Jenke-Kodama et al., 2005). It produces VLCPUFAs de novo by combined fatty acyl chain elongation, trans-cis isomerisation and enoyl reduction. Acetyl-CoA and malonyl-CoA are used as building blocks for these reactions and the whole system has a modular composition (Metz et al., 2001; Kaulmann and Hertweck, 2002). The synthesis of VLCPUFAs by the PKS-like system releases only trace amounts of intermediates which hints at a highly efficient metabolic flux (Truksa et al., 2009). On the other hand, there is the VLCPUFA biosynthesis system consisting of elongases and desaturases, which is found in marine microalgae as well as in some bacteria, fungi, non-flowering plants like mosses and animals. In Figure 3 , the pathways based on these enzymatic activities are illustrated by using the $\Delta$ nomenclature for fatty acids in order to be able to pursue the subsequent steps of desaturation and elongation. ARA is synthesized via the (n-6)-pathway from LA by sequential $\Delta 6$-desaturation, $\Delta 6$-elongation and $\triangle 5$-desaturation. Starting from ALA, but going through the same enzymatic steps, EPA is produced via the (n-3)-pathway. DHA is then built up by $\Delta 5$-elongation and $\Delta 4$-desaturation of EPA. Both (n-6)- and (n-3)pathways are interconnected by $\Delta 15$ - or $\Delta 17$-desaturases that convert ( $n$-6)-substrates into (n-3)-substrates (Pereira et al., 2004a; Damude et al., 2006). In addition to the (n6)- and (n-3)-pathways, also other variations exist. In the $\triangle 8$-pathway, LA or ALA are 
processed by a $\Delta 9$-elongase and are then further metabolized into ARA and EPA by sequential $\Delta 8$ - and $\Delta 5$-desaturation (Wallis and Browse, 1999; Qi et al., 2002; Sayanova and Napier, 2004). According to (Lang, 2007), there may be also a $\Delta 3-$ pathway, in which $18: 4(n-3)$ is desaturated at the $\Delta 3$-position and then elongated to EPA. However, to date no desaturase with $\Delta 3$-specificity could be identified. Further, the "Sprecher" pathway found exclusively in mammals is a variation to the conventional pathway of DHA synthesis from EPA. It proceeds from docosapentaenoic acid (22:5(n-3), DPA) via $\Delta 7$-elongation and $\Delta 6$-desaturation to tetracosahexaenoic acid $(24: 6(n-3))$, which is then processed via regulated peroxisomal $\beta$-oxidation into DHA (Voss et al., 1991; Sprecher et al., 1995; Sprecher et al., 1999; Sprecher, 2000). Genes encoding desaturases or elongases have been cloned from various organisms and functionally tested in plants (reviewed by (Truksa et al., 2009; Venegas-Calerón et al., 2010)).

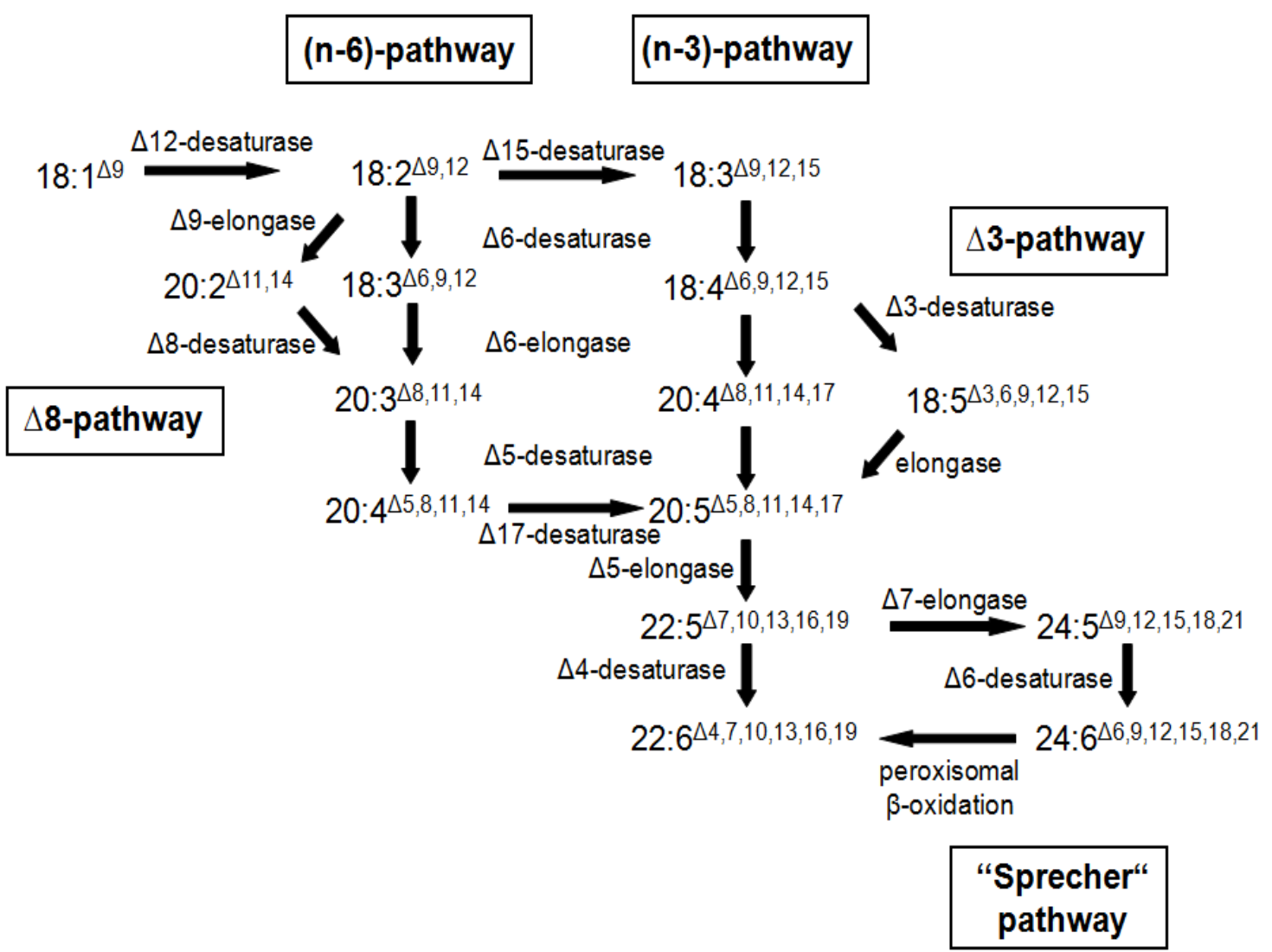

Figure 3. VLCPUFA biosynthesis pathways

(n-6)- and (n-3)-pathway as well as the $\Delta 3$-pathway and "Sprecher" pathway are illustrated. Furthermore, the alternative $\Delta 8$-pathway, which can also start from $18: 3^{\Delta 9,12,15}$ and results in $20: 4^{\Delta 8,11,14,17}$, is depicted. Figure taken from (Hoppe, 2008). 


\subsubsection{Enzymatic activities during VLCPUFA biosynthesis}

\subsubsection{Desaturases}

Fatty acid desaturases are non-heme metalloenzymes. Their active site contains a diiron center (Oshino et al., 1966; Nagai and Bloch, 1968; Jaworski, 1974; Strittmatter et al., 1974) and all of them share similarities regarding the stereochemistry of hydrogen abstraction (Shanklin and Cahoon, 1998; Sperling et al., 2003). All desaturases require oxygen (Bloomfield and Bloch, 1960; Mudd and Stumpf, 1961) and a short electron transport chain for their enzymatic activity (Fulco, 1974). In the plastid, ferredoxin is used as electron carrier for the supply with electrons either from nicotinamide adenine dinucleotide phosphate (Nagai and Bloch, 1966; Schmidt and Heinz, 1990; Wada et al., 1993) photosystem I (Jacobson et al., 1974) for the desaturation reaction. In the ER, cytochrome $b_{5}$ acts as electron carrier and receives electrons from nicotinamide adenine dinucleotide (Spatz and Strittmatter, 1971; Dailey and Strittmatter, 1979; Hackett and Strittmatter, 1984). The cytochrome $b_{5}$ can either exist as separated unit or it is already fused to the desaturase, as it is the case for so-called front-end desaturases (Sperling and Heinz, 2001).

Desaturases can be divided into two classes (Shanklin and Cahoon, 1998; Sperling et al., 2003). One class is constituted by soluble, acyl-ACP-dependent desaturases, which are located in the plastid (Bloomfield and Bloch, 1960) and have been extensively studied in the past (reviewed by (Shanklin and Cahoon, 1998)). The second class of desaturases consists of integral membrane proteins, which are difficult to access by crystallization and therefore have been analyzed by mutagenesis experiments, biochemical topology studies and comparison with related enzymes (Prasad et al., 1980; Stukey et al., 1990; Broun et al., 1998; Diaz et al., 2002; Dyer et al., 2002; Man et al., 2006b). Desaturases of this class, which also includes those desaturases required for VLCPUFA biosynthesis, contain three typical histidine boxes involved in coordination of the diiron center with consensus sequences of $\mathrm{HX}_{(3-4)} \mathrm{H}, \mathrm{HX}_{(2-3)} \mathrm{HH}$ and $H / Q X_{(2-3)} \mathrm{HH}$ (Shanklin and Cahoon, 1998). All of the histidines in this tripartite motif are necessary for catalysis (Shanklin et al., 1994). The histidine boxes are, as well as the amino- and the carboxyl-terminus of the desaturase, oriented towards the cytosolic site. From this fact it can be concluded that desaturation takes place in the cytosol (Shanklin and Cahoon, 1998).

Some of the membrane-bound desaturases involved in VLCPUFA biosynthesis are directly fused to their electron donor cytochrome $b_{5}$, as mentioned above. Aminoterminal fusions can be found in front-end desaturases from algae and mosses, carboxyl-terminal fusions are common for desaturases from fungi and yeast (Stukey et al., 1990; Sayanova et al., 1997; Itoh et al., 1998; Sperling et al., 2003). The HPGG motif forms the core of the heme-binding domain in cytochrome $b_{5}$, is highly conserved and therefore can be used as indicator for the hemeprotein (Lederer, 1994). 
Membrane-bound desaturases, neglecting some bifunctional enzymes (Hastings et al., 2001; Damude et al., 2006; Sayanova et al., 2006; Hoffmann et al., 2007), have distinct regio- and substrate-specificities (Shanklin and Cahoon, 1998). They can be classified into different subtypes concerning their location of double bond formation (Heinz, 1993). Front-end desaturases for example introduce new double bonds always between the carboxyl end of the fatty acid and an already existing double bond (Aitzetmüller and Tsevegsüren, 1994). They can have $\Delta 4-, \Delta 5-, \Delta 6-$ or $\Delta 8$-specificity (Domergue et al., 2005) and contain an $\mathrm{H}$ to $\mathrm{Q}$ substitution in their third histidine box, which is crucial for enzyme activity (Sayanova et al., 1997; Michaelson et al., 1998; Napier et al., 1998; Sayanova et al., 2001).

In contrast to the before-mentioned type, there are also the so-called $\omega$-desaturases, which position the double bond completely new or between an already existing double bond and the methyl end of the molecule. They have $\Delta 12$ - or $\Delta 15$-specificity and share a conserved HECGH motif (first histidine box) and two $\mathrm{HX}_{2} \mathrm{H}_{2}$ motifs (second and third histidine box) (Pereira et al., 2004a).

Most membrane-bound desaturases from the plant kingdom are acting on substrates that are bound to complex lipids like for example PC (Stymne and Stobart, 1986; Jackson et al., 1998; Galle-Le Bastard et al., 2000; Domergue et al., 2003) or MGDG (Norman et al., 1991). In contrast, mammalian desaturases are CoA-dependent (Okayasu et al., 1981; Irazú et al., 1993; Domergue et al., 2003), as are some recently disco-vered microalgal desaturases from $O$. tauri and Mantoniella squamata (Domergue et al., 2005; Hoffmann et al., 2008).

\subsubsection{Elongases}

Elongases are membrane-bound enzyme complexes at the ER, which condense C16 or C18 fatty acids with malonyl-CoA and thereby elongate them by two-carbon chain units. They are composed of four different proteins, which perform the sequential reactions of condensation of the acyl chain with malonyl-CoA ( $\beta$-ketoacyl-CoA synthase, $K C S$ ), reduction ( $\beta$-ketoacyl-CoA reductase, KCR), dehydration (hydroxyacyl-CoA dehydratase, HCD) and again reduction (enoyl-CoA reductases, ECR) (Fehling et al., 1992). The condensing enzyme is believed to be the determinant of substrate specificity and reaction velocity (Venegas-Calerón et al., 2010) and in previous studies, expression of KCS sequences was shown to be able to reconstitute heterologous elongation activity by interaction of the foreign KCS with the endogenous KCR, HCD and ECR activities (Millar and Kunst, 1997; Beaudoin et al., 2000; Parker-Barnes et al., 2000). The introduction of a foreign elongase into a host organism thus always refers to the respective KCS protein (Venegas-Calerón et al., 2010). In contrast to most plant desaturases, elongases act in an acyl-CoA-dependent way (Domergue et al., 2003; Jakobsson et al., 2006). 


\subsubsection{VLCPUFA production in plants}

Currently, oily sea fish and marine microalgae are the main direct nutritional sources of VLCPUFAs for humans. Both of these sources, however, comprise major disadvantages. Fish stocks are declining all over the world and the problem of overfishing will even get worse in the future due to a growing world population (Naylor et al., 2000). Furthermore, residual fish stocks are contaminated with pollutants like methyl mercury and organochlorines, which make them useless for human consumption (Hites et al., 2004). Fish farming has been considered as an adequate solution to this problem in the past, but has led to severe environmental destruction world-wide (Pauly et al., 2002). Also, aquaculture requires more input of VLCPUFAs than it gives rise to (VenegasCalerón et al., 2010). Cultivation of VLCPUFA-producing microalgae, on the other hand, requires large fermenters and is difficult to scale up (Lee, 2001). It is sensitive to changes in the power supply and holds a big environmental footprint (Venegas-Calerón et al., 2010). Taken together with an elaborate oil extraction procedure, the whole process is a very expensive and laborious approach to obtain the valuable products. For these reasons, much research has been conducted on the synthesis of VLCPUFAs in oleaginous organisms like annual oilseed crops during the last two decades. Evident advantages of VLCPUFA production in plants in this connection are the already existing infrastructures for plant cultivation and seed oil extraction with lower costs and efforts in comparison to algae cultivation and microbial oil extraction (Alonso and Maroto, 2000; Galili et al., 2002; Thelen and Ohlrogge, 2002).

Because most oilseed plants naturally synthesize only the VLCPUFA precursor fatty acids LA and ALA, several sequences encoding enzymes for the whole VLCPUFA biosynthetic pathway have to be transferred from suitable gene donors into plants and need to be expressed in a coordinated way in the desired plant tissue. Several studies were directed towards this aim in the previous years. The main findings will be presented in the following section.

Concerning the above-mentioned PKS-like system for VLCPUFA production in marine bacteria, some cloning approaches for enzymes acting in this system exist and recently, in Arabidopsis also small amounts of DHA could be produced (Metz et al., 2006). One major disadvantage of the utilization in plants might be that the PKS-like system produces free fatty acids that would require activation for lipid incorporation in seeds (Metz et al., 2009).

Regarding the alternative system employing concerted desaturation and elongation reactions for heterologous VLCPUFA production in plants, first results were published amongst others by Abbadi and co-workers (Abbadi et al., 2004). Seed-specific expression of sequences encoding a $\Delta 6$-desaturase and a $\Delta 5$-desaturase from the diatom Phaeodactylum tricornutum (Ptd6 and Ptd5, respectively) (Domergue et al., 2002) together with the sequence for the $\Delta 6$-elongase from the moss Physcomitrella patens (PSE1) (Zank et al., 2002) was performed in tobacco high in LA and linseed (Linum usitatissimum) high in ALA. With less than $1 \%$, only very low amounts of ARA and EPA were detectable. However, an accumulation of the $\Delta 6$-desaturation products 
about $25 \%$ was observed in the total lipids. At the same time, very low levels of this first intermediate of the pathway were found in the acyl-CoA pool of transgenic seeds. Taking together these data and the results from previous studies, the authors concluded that the lack of $\Delta 6$-desaturation products in the CoA pool might be due to either direct incorporation of intermediates into the TAG fraction or an insufficient substrate transfer between PC and CoA pool (Domergue et al., 2003; Abbadi et al., 2004).

An alternative approach for VLCPUFA production in plants was chosen by (Qi et al., 2004). In this study, the $\Delta 8$-pathway was established in Arabidopsis by constitutive expression of a $\Delta 9$-elongase sequence from Isochrysis galbana (Qi et al., 2002), a $\Delta 8$ desaturase sequence from Euglena gracilis (Wallis and Browse, 1999) and a $\Delta 5$ desaturase sequence from Mortierella alpina (Knutzon et al., 1998). This approach circumvented one shuttling step between PC and CoA pool and with $7 \%$ ARA and $3 \%$ EPA in leaves led to higher product yields compared to those achieved by Abbadi and co-workers (Qi et al., 2004).

Substantial increases in product levels as well as DHA production in plants could be attained by Kinney et al. in the same year (Kinney et al., 2004). Soybean somatic embryos were transformed with one vector containing sequences from $M$. alpina encoding the enzymatic activities for EPA production and in addition with a second vector containing sequences for a $\Delta 17$-desaturase from Saprolegnia diclina (Pereira et al., 2004a), a $\Delta 5$-elongase sequence from Pavlova salina (Pereira et al., 2004b) and a $\Delta 4$ desaturase sequence from Schizochytrium aggregatum. Up to $3.3 \%$ DHA was found in the total fatty acids and up to $20 \%$ EPA was detectable in seeds from plants deriving from transformation with the EPA construct (Kinney et al., 2004).

In a study conducted by (Wu et al., 2005) in Indian mustard, a series of binary vectors containing three to nine genes all controlled by the same seed-specific promoter was used for the stepwise addition of enzymatic activities to be able to follow the metabolic flux in plants. ARA yields averaged $18 \%$, EPA yields were up to $8 \%$ and DHA yields were about $0.2 \%$ of total fatty acids. Wu and co-workers made observations that were similar to the findings described by Abbadi et al. (2004), but could enhance elongation rates by expression of a second elongase sequence. From these data the authors concluded that substrate availability is not the only limiting factor for elongation efficiency (Wu et al., 2005).

Both last-mentioned studies thus led to higher product yields in soybean and Indian mustard compared to linseed and tobacco. Therefore, it can be concluded that the utilized host plant and its endogenous acyltransferase activities have a great impact on successful VLCPUFA production (Napier, 2007a). This fact was also reinforced by complementary experiments conducted by Abbadi and co-workers. In these studies, the same enzymatic activities used before were introduced into a linseed type rich in LA as well as in rapeseed. ARA or ARA and EPA, respectively, accumulated strongly in transgenic seeds. The accumulation of $\Delta 6$-desaturation products was lower and the elongation rates were higher compared to the data obtained in tobacco and linseed high in ALA (Abbadi et al., 2007). 
In a first approach to enhance VLCPUFA yields in plants by utilization of an exclusively acyl-CoA-dependent pathway, $A$. thaliana was transformed with a construct containing a sequence encoding a bifunctional acyl-CoA-dependent $\Delta 6 / \Delta 5$-desaturase from zebrafish (Danio rerio) (Hastings et al., 2001) and a $\Delta 6$-elongase sequence from Caenorhabditis elegans (Beaudoin et al., 2000). Expression led up to $1.6 \%$ ARA and $3.2 \%$ EPA of total fatty acids. Plants were retransformed with a $\Delta 5$-elongase and a $\Delta 4$ desaturase from $P$. salina, which led up to $0.5 \%$ DHA (Robert et al., 2005). However, the heterologous expression of genes from animals in plants always evokes ethical questions and is not accepted by the general public (Napier, 2007b). Therefore, the utilization of enzymes from the plant kingdom would be favourable. This aim could recently be achieved by (Hoffmann et al., 2008). In this study, sequences encoding acylCoA-dependent desaturases could be identified in the microalgal species M. squamata. They were co-expressed together with PSE1 (Zank et al., 2002) in Arabidopsis under the control of a seed-specific promoter. These experiments could demonstrate the circumvention of the transfer bottleneck after the first desaturation step, because the accumulation of $\Delta 6$-desaturation products could not be observed any more. Due to the low activity of the identified $\Delta 5$-desaturase, however, EPA yields were comparable to the ones obtained with the lipid-dependent desaturases, which had also been used by (Abbadi et al., 2004).

In summary, EPA yields achieved by heterologous expression of genes from diverse gene donor organisms are already quite satisfying. For DHA production, however, still some improvements concerning metabolic flux need to be done (Truksa et al., 2009). This could for example be achieved by the identification of an acyl-CoA-dependent $\Delta 4$ desaturase, that supersedes the requirement of shuttling between PC and CoA pool also in this last part of the (n-3)-pathway. 


\subsection{Aims of the project}

The experiments in the presented thesis were performed to continue preceding studies on the identification and characterization of enzymes directly or indirectly involved in microalgal VLCPUFA biosynthesis.

In studies conducted by Dr. M. Wagner, different sequences putatively encoding acyltransferases were identified in the microalgal species $O$. tauri and O. lucimarinus and characterized in parts (Wagner, 2008). Their substrate specificities as well as their possible interaction should be further investigated by in vitro and in vivo experiments in yeast.

As described in the previous section, Dr. M. Heilmann could identify two exclusively acyl-CoA-dependent desaturases with $\Delta 6$ - and $\Delta 5$-specificity from $M$. squamata and expressed their sequences together with the elongase sequence PSE1 in Arabidopsis. By this approach, she could establish EPA biosynthesis in planta and circumvented the bottleneck between desaturation and elongation described by Abbadi and co-workers (Hoffmann et al., 2008). The next logical step would be the synthesis of DHA by introduction of an additional elongation and a desaturation activity. A few elongases with $\Delta 5$-specificity have already been isolated from microalgae (Meyer et al., 2003; Pereira et al., 2004b). Thus, the aims of this work were to identify an acyl-CoA-dependent desaturase with $\Delta 4$-specificity, to characterize the enzyme by heterologous expression of its encoding sequence in yeast and to check its suitability for utilization in planta.

Last but not least, putative microalgal acyltransferases should be introduced into seed lipid metabolism of wild type and mutant plants to examine their effects in planta. Also, VLCPUFA production in plants should be further investigated by co-expression of different desaturase and elongase sequences at hand. VLCPUFA yields should be compared in consecutive transgenic plant generations possessing various combinations of desaturation and elongation activities and the interplay of desaturases and elongases should be compared in different plant species. Furthermore, desaturase, elongase and acyltransferase sequences should be co-expressed in Arabidopsis to test possible acyltransferase effects on VLCPUFA yields. 


\section{Material and Methods}

Basic molecular biological and biochemical techniques were performed as described in (Ausubel et al., 1993). For all methods sterile pipet tips and reaction tubes were used. All solutions were set up with double distilled water $\left(\mathrm{ddH}_{2} \mathrm{O}\right)$ and sterilized by autoclaving them for $15-20$ minutes $(\min )$ at $120^{\circ} \mathrm{C}$.

\subsection{Technical Equipment}

ABI PRISM 3100 genetic analyzer

Biofuge pico

Centrifuge $5810 \mathrm{R}$

Centrifuge $5415 \mathrm{D}$

Centrifuge $5417 \mathrm{R}$

Chromatogram immersion device III

Clean bench Prettl-Telstar Bio-II-A

6890 series gas chromatograph

6890 gas chromatograph/

5973 mass selective detector system

Gel detection system IDA

1100 series HPLC system

Mastercycler gradient

Mastercycler personal

Mini-PROTEAN 3 system

Mini Trans-Blot cell equipment

Optima L-80K ultracentrifuge

Optimax Typ TR developer

Percival climate chamber

Phenyl-hexyl column

(5 $\mu \mathrm{m}, 150 \mathrm{~mm} \times 2 \mathrm{~mm}$ )

Phospho- and fluorescence-imager

Fuji FLA-3000

TLC heating plate

TLC spray cabinet/sprayer

Ultrospec 1100 pro
Applied Biosystems (Foster, USA)

Heraeus Sepatech (Osterode, Germany)

Eppendorf AG (Hamburg, Germany)

Eppendorf AG (Hamburg, Germany)

Eppendorf AG (Hamburg, Germany)

Camag (Muttenz, Switzerland)

Telstar (Terrass, Spain)

Agilent (Waldbronn, Germany)

Agilent (Waldbronn, Germany)

Raytest (Straubenhardt, Germany)

Agilent (Waldbronn, Germany)

Eppendorf AG (Hamburg, Germany)

Eppendorf AG (Hamburg, Germany)

Biorad (Hercules, CA, USA)

Biorad (Hercules, CA, USA)

Beckman Coulter (Krefeld, Germany)

MS Labware (Wiesloch bei Heidelberg,

Germany)

CLF Plant Climatics (Emersacker, Germany)

Phenomenex (Aschaffenburg, Germany)

Raytest (Straubenhardt, Germany)

Camag (Muttenz, Switzerland)

Camag (Muttenz, Switzerland)

GE Healthcare (Freiburg, Germany) 


\subsection{Chemicals and Standards}

All chemicals, if not mentioned otherwise, were purchased from Sigma (Munich, Germany), Merck (Darmstadt, Germany), Carl Roth \& Co. (Karlsruhe, Germany), Invitrogen (Karlsruhe, Germany) or Duchefa Biochemistry (Haarlem, Netherlands). All solvents of high performance liquid chromatography grade for the analytical methods were purchased from Acros (Geel, Belgium) or Baker (Griesheim, Germany). Fatty acid substrates were ordered from Cayman Chemical (Ann Arbor, MI, USA). Fatty acid standards were from Sigma (Munich, Germany) and acyl-CoA standards from Larodan (Malmö, Sweden). Radiolabeled acyl-CoAs were ordered from American Radiolabeled Chemicals (St. Louis, MO, USA).

\subsection{Enzymes}

\section{Restriction enzymes}

Restriction enzymes Ascl, Apal, BamHI, EcoRl, HindIII, Kpnl, Nhel, Notl, Spel and Xhol were ordered from Fermentas (St. Leon Rot, Germany), restriction enzyme Sbfl from was from New England Biolabs (Ipswich, UK). All of them were used according to the manufacturer's instructions.

\section{Other enzymes}

Advantage PCR Enzyme System

Calf intestine alkaline phosphatase

DNAse I

TaKaRa ExTaq DNA polymerase

Phusion DNA polymerase

T4 DNA ligase

\subsection{Kits and Systems}

ABI Prism BigDye Terminator Cycle Sequencing Ready Reaction Kit v1.1

ECL Western blotting detection reagents and analysis system Gateway LR Clonase II Enzyme Mix NucleoSpin Extract Kit

NucleoSpin Plasmid Kit

RevertAid H Minus First Strand cDNA synthesis kit
Clontech (Mountain View, CA, USA)

Fermentas (St. Leon Rot, Germany)

Fermentas (St. Leon Rot, Germany)

Cambrex BioScience (Potsdam, Germany)

Finnzymes (Espoo, Finland)

Fermentas (St. Leon Rot, Germany)
Applied Biosystems, Foster, USA

GE Healthcare (Freiburg, Germany)

Invitrogen (Karlsruhe, Germany)

Macherey-Nagel (Düren, Germany)

Macherey-Nagel (Düren, Germany)

Fermentas (St. Leon Rot, Germany) 


\subsection{Vectors}

\section{Subcloning vectors}

pGEM-T

$\mathrm{Amp}^{\mathrm{R}}$ (Promega, Madison, WI, USA)

pUC18-Entry2

$\mathrm{Amp}^{\mathrm{R}}$ (provided by Dr. E. Hornung)

pUC18-Entry2 contains a multiple cloning site with inserted $c c d B$ gene for selection of positive transformants and flanking att $L 1$ and att $L 2$ sites for gateway cloning.

\section{Yeast expression vectors}

pESC-TRP

pESC-LEU

PYES2/CT

pUG36
$A m p^{R}$ (Stratagene, La Jolla, CA, USA)

$\mathrm{Amp}^{\mathrm{R}}$ (Stratagene, La Jolla, CA, USA)

$A m p^{R}$ (Invitrogen, Karlsruhe, Germany)

$\mathrm{Amp}^{\mathrm{R}}$ (by courtesy of Dr. R. Krick)

pUG36 was created by Dr. U. Güldener and Prof. Dr. J. H. Hegemann (http://mips.helmholtz-muenchen.de/proj/yeast/info/tools/hegemann/gfp.html).

\section{Plant expression vectors}

pCAMBIA3300

$\operatorname{Kan}^{R}$ (bacteria), glufosinate ${ }^{R}$ (plants)

(provided by Dr. Andreas Hiltbrunner, ETH

Zürich, Switzerland; with modifications

according to (Abbadi et al., 2004))

pCAMBIA33.2cGs

$\mathrm{Kan}^{\mathrm{R}}$ (bacteria), glufosinate ${ }^{\mathrm{R}}$ (plants)

(provided by Dr. E. Hornung)

The pCAMBIA vectors contain the bar gene under the control of the constitutive Cauliflower mosaic virus $35 \mathrm{~S}$ promoter. pCAMBIA3300 contains Sbfl and Ascl restriction sites. pCAMBIA33.2cGs contains the seed-specific LeB4 promoter (Bäumlein et al., $1991 \mathrm{~b})$ behind attR1 and attR2 sites for gateway cloning. 


\subsection{DNA constructs}

\section{Subcloning constructs}

Ptd6-PSE1-Ptd5-pUC19-USP-OCS123

(Sbfl)

Msd6-pUC19-USP-OCS123 (Ascl)

provided by Dr. M. Wagner

Msd6-OILPCAT-pUC19-USP-OCS123

$(A s c l)$

OILPCAT-pUC19-USP-OCS123 (Ascl)

pUC19-USP-OCS123 (Abbadi et al., 2004) with modifications) was used for subcloning of cDNAs that should subsequently be brought into pCAMBIA3300. It contains three different polylinkers between three seed-specific USP promoters (Bäumlein et al., 1991a) and three OCS terminators (Macdonald et al., 1991). This three-way expression cassette can be cut out of the pUC vector by restriction digest either with Ascl or Sbfl.

\section{Yeast expression constructs}

OtDGAT2A-pYES2/CT

OtDGAT2B-pYES2/CT

OtDGAT2C-pYES2/CT

OILPCAT-pYES2/CT

OILPCAT-V5-pYES2/CT

DGA1-pYES2/CT

OtPDAT-pYES2/CT

AtDGAT1-pBT3N

AtDGAT2-pBT3N

OtLPCAT-pESC-TRP

mCherryFAR1-pESC-URA

WS-mCherryFAR1-pESC-URA

ALE1-pYES2.1/TOPO

LacZ-V5-pYES2/CT

provided by Dr. M. Wagner

(Wagner, 2008)

provided by Dr. M. Heilmann

by courtesy of Dr. U. Ståhl

(Stahl et al., 2008)

Invitrogen (Karlsruhe, Germany)

cDNA sequences for OtDGAT2A, OtDGAT2C and OILPCAT were completely codonoptimized for optimal expression in yeast and Arabidopsis. In cDNA sequences for Ot$D G A T 2 B$ and OtPDAT codons 1-25 were optimized. The process of codon-optimization is described in (Wagner, 2008). In case of OtLPCAT, codons 1-14 were optimized. 
FAR1 is a fatty acid reductase sequence from mouse (Cheng and Russell, 2004a), WS is a wax synthase sequence from mouse (Cheng and Russell, 2004b).

\section{Plant expression constructs}

Msd6-PSE1-Msd5-OtELO5-Egd4-

pCAMBIA3300

provided by S. Schlenczek

Ptd6-PSE1-Ptd6-pCAMBIA3300

provided by Dr. M. Heilmann

(Hoffmann et al., 2008)

\subsection{Antibodies}

\section{Primary antibodies}

Anti-V5-mouse

Invitrogen (Karlsruhe, Germany)

Anti-GFP-mouse

Covance (Munich, Germany)

\section{Secondary antibodies}

Anti-mouse-peroxidase

Sigma (Munich, Germany)

\subsection{Software for Sequence Analyses}

For identification of new putative desaturase sequences the databases of the National Center of Biotechnology Information (NCBI) were scanned using the Basic Local Alignment Tool (BLAST) algorithm (Altschul et al., 1997) with already known front-end desaturases as queries (http://www.ncbi.nlm.nih.gov/blast/Blast.cgi). The identified nucleotide sequence was then further analyzed by similarity searches in the NCBI databases using the BLAST program. Hits with experimentally proven substrate specificity were chosen for global pairwise alignment using EMBOSS Pairwise Alignment Algorithms (http://www.ebi.ac.uk/emboss/align/; needle-algorithm; Blosum62 matrix) (Rice et al., 2000). Multiple alignments of DNA or nucleotide sequences were done using the program MultAlin (http://bioinfo.genopole-toulouse.prd.fr/multalin/multalin.html) (Corpet, 1988). Boxshade 3.21 (http://www.ch.embnet.org/software/BOX_form.html) was then used to display the results. For protein localization predictions, the programs WoLF PSORT (http://www.wolfpsort.org) (Horton et al., 2007) was used, for prediction of $\begin{array}{llll}\text { chloroplast } \quad \text { transit } & \text { peptides } & \text { (cTPs), } & \text { ChloroP1.1 }\end{array}$ (http://www.cbs.dtu.dk/services/ChloroP/) (Emanuelsson et al., 1999) was applied. The program ProtScale (http://www.expasy.ch/tools/protscale.html) (Gasteiger et al., 2005) was used to predict the molecular weight of proteins. Phylogenetic trees were generated using the CLUSTALX multiple alignment program (Thompson et al., 1997) and the program Phylip (Felsenstein, 2005). The program Treellustrator was used to display the resulting phylograms (Trooskens et al., 2005). 


\subsection{Organisms}

\subsubsection{Algae}

Ostreococcus lucimarinus (CCMP2972) was obtained from the Provasoli-Guillard National Center for Culture of Marine Phytoplankton (CCMP, West Boothbay Harbor, Maine, USA). Thalassiosira pseudonana (1020-1b) was provided by the culture collection of algae Göttingen (SAG, Göttingen, Germany).

\subsubsection{Bacteria}

Escherichia coli XL1blue

Agrobacterium tumefaciens EHA105

A. tumefaciens C58C1
Genotype: endA1, hsdR17, supE44, thi-1, rec $A 1$, gyrA96, relA1, lac, $\left[F^{\prime}\right.$, proAB, lacl ${ }^{q} Z \Delta 15, \operatorname{Tn} 10\left(\right.$ tet $\left.^{R}\right)$ ]; Stratagene (La Jolla, CA, USA) (Bullock et al., 1987)

Genotype: pTiBo542 $\Delta$ T-DNA Rif ${ }^{R}$ (Hood et al., 1993)

Genotype: pTiBo542 $\Delta$ T-DNA Rif ${ }^{R}$, derivative of the strain EHA101 (Hood et al., 1993; Hellens et al., 2000), transformed with Ptd6PSE1-Ptd5-pCAMBIA2200 (Camp ${ }^{R}$ (bacteria), $\operatorname{Kan}^{\mathrm{R}}$ (plants); (Abbadi et al., 2004))

\subsubsection{Yeast}

S. cerevisiae INVSc1

S. cerevisiae BY4741

S. cerevisiae BY4741 ale1A

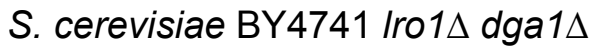

Genotype: his3 $\Delta 1 /$ his $3 \Delta 1$ leu2/leu2 trp1289/trp1-289 ura3-52/ura3-52 (Invitrogen, Karlsruhe, Germany)

Genotype: MATa his $3 \Delta 1$ leu2 $\Delta 0$ met15 $\Delta 0$ ura3 $\triangle O$ (Brachmann et al., 1998)

Genotype: as above; ale1- $\Delta:: k a n M X 4$ (Euroscarf, Frankfurt, Gemany)

Genotype: as above; Iro1- $\Delta:: k a n M X 4$ dga1- $\Delta::$ natMX4 (provided by Dr. M. Wagner)

S. cerevisiae BY4741 Iro1 $\Delta$ dga1 $\Delta$ ale1 $\Delta$ Genotype: as above; Iro1- $\Delta:: k a n M X 4$ dga1- $\Delta:: n a t M X 4$ ale1- $\Delta:: h p h M X 4$ (this work)

S. cerevisiae BY4741 Iro1 $\Delta$ dga1 $\Delta$ tes1 $\Delta$ Genotype: as above; Iro1- $\Delta:$ kanMX4 dga1- $\Delta:: n a t M X 4$ tes $1-\Delta:: h p h M X 4$ (this work) 
S. cerevisiae W303 H1246

Genotype: MATa ade2-1 can1-100 his311,15 leu2-3, 112 trp1-1 ura3-1 are1- $\triangle:: H I S 3$ are2- $\triangle:: L E U 2$ dga1- $\Delta:: K a n M X 4$ Iro1- $\Delta:: T R P 1$ $A D E 2$ (Sandager et al., 2002), by courtesy of Prof. Dr. S. Stymne)

\subsubsection{Plants}

Arabidopsis thaliana L var. Columbia (Col-0)

A. thaliana $L$ var. Columbia (Col-0) tag1-1 mutant (AS11; (Katavic et al., 1995))

A. thaliana $L$ var. Columbia (Col-0) transformed with

Ptd6-PSE1-Ptd5-pCAMBIA3300

Msd6-PSE1-Msd5-pCAMBIA3300

Otd6-PSE1-Otd5-pCAMBIA3300

provided by Dr. M. Heilmann (Hoffmann et al., 2008)

Camelina sativa L.Crantz subsp. Ligena

\subsection{Cultivation conditions}

\subsubsection{Algae}

O. Iucimarinus cultures were grown as batch cultures in $200 \mathrm{ml}$ sterile liquid L1 medium, T. pseudonana cultures were grown as batch cultures in $200 \mathrm{ml}$ sterile liquid $1 / 2$ SWES medium with $1 \%$ silicate. All cultures were cultivated at $20^{\circ} \mathrm{C}, 110 \mu \mathrm{mol}$ photons ${ }^{*} \mathrm{~m}^{-2 *} \mathrm{~s}^{-1}$ and a day length of 14 hours in a Percival climate chamber (CLF Plant Climatics).

L1 medium (Guillard, 1993)

$\begin{array}{ll}75 \mathrm{mg} / \mathrm{l} & \mathrm{NaNO}_{3} \\ 5 \mathrm{mg} / \mathrm{l} & \mathrm{NaH}_{2} \mathrm{PO}_{4}{ }^{*} \mathrm{H}_{2} \mathrm{O} \\ 30 \mathrm{mg} / \mathrm{l} & \mathrm{Na}_{2} \mathrm{SiO}_{3}{ }^{*} \mathrm{H}_{2} \mathrm{O} \\ 1 \mathrm{ml} / \mathrm{l} & \mathrm{L} 1 \text { trace element solution } \\ 0.5 \mathrm{ml} / \mathrm{l} & \mathrm{f} / 2 \text { vitamin solution }\end{array}$

The medium was set up in filtered seawater (Biologische Anstalt Helgoland, Germany) and autoclaved at $120^{\circ} \mathrm{C}$ for $20 \mathrm{~min}$. The vitamin solution was added afterwards, the medium was stored at $4{ }^{\circ} \mathrm{C}$. 


\section{L1 trace element solution}

Stock solution $(g / l)$

Quantity

$\mathrm{Na}_{2}$ EDTA $^{*} 2 \mathrm{H}_{2} \mathrm{O}$

$4.36 \mathrm{~g}$

$\mathrm{FeCl}_{3}{ }^{*} 6 \mathrm{H}_{2} \mathrm{O}$

$3.15 \mathrm{~g}$

$\mathrm{MnCl}_{2}{ }^{*} 4 \mathrm{H}_{2} \mathrm{O}$

178.1

$1 \mathrm{ml}$

$\mathrm{ZnSO}_{4}{ }^{*} 7 \mathrm{H}_{2} \mathrm{O}$

23

$1 \mathrm{ml}$

$\mathrm{CoCl}_{2}{ }^{*} 6 \mathrm{H}_{2} \mathrm{O}$

$1 \mathrm{ml}$

$\mathrm{CuSO}_{4}{ }^{*} 5 \mathrm{H}_{2} \mathrm{O}$

$1 \mathrm{ml}$

$\mathrm{Na}_{2} \mathrm{MoO}_{4}{ }^{*} 2 \mathrm{H}_{2} \mathrm{O}$

19.9

$1 \mathrm{ml}$

1.29

$1 \mathrm{ml}$

2.63

$1 \mathrm{ml}$

1.84

$1 \mathrm{ml}$

1.94

$1 \mathrm{ml}$

The solution was set up in $d_{d d} \mathrm{H}_{2} \mathrm{O}$, autoclaved at $120^{\circ} \mathrm{C}$ for 20 min and stored at $4{ }^{\circ} \mathrm{C}$.

f/2 vitamin solution (Guillard and Ryther, 1962)

$1 \mathrm{mg} / \mathrm{l} \quad$ Cyanocobalamine (vitamin $\mathrm{B}_{12}$ )

$1 \mathrm{mg} / \mathrm{l} \quad$ Biotin (vitamin $\mathrm{H}$ )

$100 \mathrm{mg} / \mathrm{l}$ Thiamine chloride (vitamin $\mathrm{B}_{1}$ )

The solution was set up in $d_{d H_{2}} \mathrm{O}$, filter sterilized and stored at $4{ }^{\circ} \mathrm{C}$.

\section{$1 / 2$ SWES medium with $1 \%$ silicate}

$0.2 \mathrm{~g} / \mathrm{l} \quad \mathrm{KNO}_{3}$

$20 \mathrm{mg} / \mathrm{l} \mathrm{MgSO}_{4}{ }^{*} 7 \mathrm{H}_{2} \mathrm{O}$

$20 \mathrm{mg} / \mathrm{l} \quad \mathrm{KH}_{2} \mathrm{PO}_{4}$

$30 \mathrm{ml} / \mathrm{l} \quad$ Soil extract ${ }^{\#}$

$5 \mathrm{ml} / \mathrm{l} \quad$ Micronutrient solution

$450 \mathrm{ml} / \mathrm{l} \quad \mathrm{ddH}_{2} \mathrm{O}$

$455 \mathrm{ml} / \mathrm{l} \quad$ Filtered seawater (Biological Institute Helgoland, Germany)

$50 \mathrm{ml} / \mathrm{l} \quad$ Saturated solution of $\mathrm{Na}_{2} \mathrm{SiO}_{3}{ }^{*} 9 \mathrm{H}_{2} \mathrm{O}$

\#Soil extract was produced from garden soil. The soil was mixed with water in the ratio 1:2 and autoclaved three times at $120{ }^{\circ} \mathrm{C}$ for $20 \mathrm{~min}$. The supernatant was filtered through a round filter (Whatman $\mathrm{GmbH}$, Dassel, Germany) and the solution was stored at $4{ }^{\circ} \mathrm{C}$. 
The medium was autoclaved at $120^{\circ} \mathrm{C}$ for $20 \mathrm{~min}$ and cooled down. Then sterile filtrated vitamin $B_{12}(5 \mu \mathrm{g} / \mathrm{l})$ was added. The solution was stored at $4{ }^{\circ} \mathrm{C}$.

\section{Micronutrient solution}

I

$\mathrm{ZnSO}_{4}{ }^{*} 7 \mathrm{H}_{2} \mathrm{O}$

$\mathrm{MnSO}_{4}{ }^{*} 4 \mathrm{H}_{2} \mathrm{O}$

$\mathrm{H}_{3} \mathrm{BO}_{3}$

$\mathrm{Co}\left(\mathrm{NO}_{3}\right)_{2}{ }^{*} 6 \mathrm{H}_{2} \mathrm{O}$

$\mathrm{Na}_{2} \mathrm{MoO}_{4}{ }^{*} 2 \mathrm{H}_{2} \mathrm{O}$

$\mathrm{CuSO}_{4} * 5 \mathrm{H}_{2} \mathrm{O}$

$\mathrm{ddH}_{2} \mathrm{O}$

\section{II}

$\mathrm{FeSO}_{4}{ }^{*} 7 \mathrm{H}_{2} \mathrm{O}$

0.7

EDTA

0.8

$\mathrm{ddH}_{2} \mathrm{O}$

\section{Stock solution $(\mathrm{g} / 100 \mathrm{ml})$}

0.1

0.1

0.2

0.02

0.02

0.0005

Both solutions were set up separately in $\mathrm{ddH}_{2} \mathrm{O}$ to avoid precipitations. They were autoclaved at $120^{\circ} \mathrm{C}$ for $20 \mathrm{~min}$, then combined and stored at $4{ }^{\circ} \mathrm{C}$.

\subsubsection{Bacteria}

E. coli XL1blue cells were grown at $37^{\circ} \mathrm{C}$ in liquid Luria and Bertani (LB) medium while shaking at $200 \mathrm{rpm}$ or on solid LB medium. A. tumefaciens cells were grown at $28^{\circ} \mathrm{C}$ in liquid YEB medium while shaking at $200 \mathrm{rpm}$ or on solid YEB medium. For the production of solid medium $1.5 \%$ agar (w/v) (Duchefa Biochemistry, Haarlem, Netherlands) was added to liquid medium. All media were set up in $\mathrm{ddH}_{2} \mathrm{O}$ and autoclaved at $120{ }^{\circ} \mathrm{C}$ for 20 min. For selection purposes different media additives were added.

LB medium (Silhavy et al., 1984)

$\begin{array}{ll}10 \mathrm{~g} / \mathrm{l} & \text { Peptone } \\ 5 \mathrm{~g} / \mathrm{l} & \text { Yeast Extract } \\ 10 \mathrm{~g} / \mathrm{l} & \mathrm{NaCl}\end{array}$




\section{YEB medium}

$\begin{array}{ll}5 \mathrm{~g} / \mathrm{l} & \text { Beef extract } \\ 1 \mathrm{~g} / \mathrm{l} & \text { Peptone } \\ 1 \mathrm{~g} / \mathrm{l} & \text { Yeast Extract } \\ 5 \mathrm{~g} / \mathrm{l} & \text { Sucrose } \\ 5 \mathrm{mM} & \text { MgSO }_{4}{ }^{*} 7 \mathrm{H}_{2} \mathrm{O}\end{array}$

\section{Media additives}

$\begin{array}{cccc} & \text { Solvent } & \text { Stock solution } & \text { End concentration } \\ \text { Carbenicillin } & \text { dd }_{2} \mathrm{O} & 100 \mathrm{mg} / \mathrm{ml} & 100 \mathrm{mg} / \mathrm{l} \\ \text { Kanamycin } & \mathrm{ddH}_{2} \mathrm{O} & 50 \mathrm{mg} / \mathrm{ml} & 25 \mathrm{mg} / \mathrm{l} \\ \text { Rifampicin } & \text { Dimethylsulfoxide (DMSO) } & 50 \mathrm{mg} / \mathrm{ml} & 50 \mathrm{mg} / \mathrm{l} \\ \text { IPTG } & \text { dd }_{2} \mathrm{O} & 0.1 \mathrm{M} & 0.2 \mathrm{mM} \\ \text { X-Gal } & \text { Dimethylformamide (DMF) } & 2 \%(\mathrm{w} / \mathrm{v}) & 0.004 \%(\mathrm{w} / \mathrm{v}) \\ \text { Tetracycline } & \text { Ethanol } & 10 \mathrm{mg} / \mathrm{ml} & 10 \mathrm{mg} / \mathrm{l}\end{array}$

\subsubsection{Yeast}

All S. cerevisiae strains were grown in liquid or on solid YPD or SD medium at temperatures between $16{ }^{\circ} \mathrm{C}$ and $30{ }^{\circ} \mathrm{C}$ as indicated for each experiment. Liquid cultures were shaken at 150-200 rpm. For production of solid medium $2 \%$ (w/v) agar (Duchefa Biochemistry, Haarlem, Netherlands) were added to liquid medium. All media were set up in $\mathrm{ddH}_{2} \mathrm{O}$ and autoclaved at $120^{\circ} \mathrm{C}$ for $15 \mathrm{~min}$.

YPD medium (complete medium)

$10 \mathrm{~g} / \mathrm{l} \quad$ Yeast Extract

$20 \mathrm{~g} / \mathrm{l} \quad$ Peptone

$20 \mathrm{~g} / \mathrm{l} \quad$ Glucose Monohydrate

For selection purposes, hygromycin B was added to a final concentration of $300 \mu \mathrm{g} / \mathrm{ml}$ after autoclaving the medium.

SD medium (selection medium)

$1.7 \mathrm{~g} / \mathrm{l} \quad$ Yeast Nitrogen Base (MP Biomedicals, Heidelberg, Germany) $2.5 \mathrm{~g} / \mathrm{l} \quad\left(\mathrm{NH}_{4}\right)_{2} \mathrm{SO}_{4}$

After autoclaving and before use, $2 \%(\mathrm{w} / \mathrm{v})$ sugar (sterile filtrated glucose, raffinose or galactose) as well as synthetic complete drop-out medium mix to a final concentration of $1 \mathrm{x}$ were added. 


\section{0 x Synthetic complete drop-out medium mix}

$\begin{array}{ll}2 \mathrm{~g} & \text { Adenine hemisulfate } \\ 2 \mathrm{~g} & \text { L-Arginine } \mathrm{HCl} \\ 2 \mathrm{~g} & \text { L-Histidine } \mathrm{HCl} \\ 2 \mathrm{~g} & \text { L-Isoleucine } \\ 4 \mathrm{~g} & \text { L-Leucine } \\ 2 \mathrm{~g} & \text { L-Lysine } \mathrm{HCl} \\ 2 \mathrm{~g} & \text { L-Methionine } \\ 3 \mathrm{~g} & \text { L-Phenylalanine } \\ 2 \mathrm{~g} & \text { L-Serine } \\ 2 \mathrm{~g} & \text { L-Threonine } \\ 3 \mathrm{~g} & \text { L-Tryptophan } \\ 2 \mathrm{~g} & \text { L-Tyrosine } \\ 1.2 \mathrm{~g} & \text { Uracil } \\ 9 \mathrm{~g} & \text { L-Valine }\end{array}$

Substances were mixed, leaving out the respective component for selection, and ground. They were resuspended to a concentration of $100 \mathrm{~g} / \mathrm{l}$ in sterile $\mathrm{ddH}_{2} \mathrm{O}$ and stored at $4{ }^{\circ} \mathrm{C}$.

\subsubsection{Plants}

\subsubsection{Surface sterilization of $A$. thaliana seeds}

Prior to sterile cultivation of $A$. thaliana plants on plates, seeds were sterilized. For a $150 \mathrm{~mm}$ plate, $40 \mathrm{mg}$ of seeds were mixed with $1 \mathrm{ml} 6 \%$ (w/v) NaOCl containing $0.1 \%$ $(\mathrm{v} / \mathrm{v})$ Triton X-100 in sterile tubes and were incubated on a rocking table for $15 \mathrm{~min}$. Supernatants were discarded and seeds were washed four times with $1 \mathrm{ml}$ of sterile $\mathrm{ddH}_{2} \mathrm{O}$. Afterwards, seeds were resuspended in 6-8 $\mathrm{ml} 0.1 \%(\mathrm{w} / \mathrm{v})$ agar.

\subsubsection{Cultivation on plates}

Surface-sterilized seeds were sown onto plates containing 1/2 Murashige Skoog (MS) medium. For selection of transgenic plants, kanamycin was added to a final concentration of $40 \mu \mathrm{g} / \mathrm{ml}$ after autoclaving. For freshly transformed seeds, also cefotaxime was added to a final concentration of $100 \mu \mathrm{g} / \mathrm{ml}$ to prevent agrobacteria from growing on the plates. Plates were wrapped with $3 \mathrm{M}$ micropore tape and then incubated at $23{ }^{\circ} \mathrm{C}$ and under constant illumination of $120 \mu \mathrm{E}$. 


\section{$1 / 2$ MS medium}

$2.2 \mathrm{~g} / \mathrm{l} \quad$ Murashige Skoog powder

$1 \%(w / v)$ Sucrose

$7 \mathrm{~g} / \mathrm{l} \quad$ Microagar

The medium was set up in $\mathrm{ddH}_{2} \mathrm{O}$, adjusted to $\mathrm{pH} 5.9$ with $\mathrm{KOH}$ and then autoclaved for $20 \mathrm{~min}$ at $120^{\circ} \mathrm{C}$.

\subsubsection{Cultivation on soil}

Prior to usage, soil was incubated for 8 hours at $180{ }^{\circ} \mathrm{C}$ to reduce fungal contaminations. A. thaliana seeds were sown non-sterile on soil (Frühstorfer Erde Typ EP Nr. 340, Industrie Erdwerk Archut, Lauterbach-Wallenrod, Germany) and incubated for stratification 2-4 days at $4{ }^{\circ} \mathrm{C}$. Afterwards, plants were either cultivated in the greenhouse at a day length of 16 hours and temperatures between $16-22{ }^{\circ} \mathrm{C}$ or in the climate chamber at a day length of 16 hours $\left(120 \mu \mathrm{mol} / \mathrm{m}^{2} \mathrm{~s}\right)$, air humidity of $60 \%$ and a temperature of $22{ }^{\circ} \mathrm{C}$. C. sativa plants were grown non-sterile on soil (Frühstorfer Erde Typ T 25, Industrie Erdwerk Archut, Lauterbach-Wallenrod, Germany) in the greenhouse at a day length of 16 hours and temperatures between $16-22{ }^{\circ} \mathrm{C}$. The herbicide Basta (Bayer CropScience, Monheim, Germany) containing glufosinate as active ingredient was used for selection of transgenic plants and sprayed one and two weeks after sowing onto the plants.

\subsection{Molecular cloning methods}

\subsubsection{Isolation of RNA from algae cultures or plant material}

Total ribonucleic acid (RNA) was isolated using the protocol described by (Vicient and Delseny, 1999). Prior to usage, solutions for RNA isolation were treated with $0.1 \%$ $(\mathrm{v} / \mathrm{v})$ diethylpyrocarbonate (DEPC) for at least 1 hour at $37^{\circ} \mathrm{C}$ to inactivate RNAses. Then, solutions were autoclaved for $20 \mathrm{~min}$ at $120^{\circ} \mathrm{C}$ to destroy residual DEPC. Algal material was harvested by centrifugation at $4{ }^{\circ} \mathrm{C}$ and 3320 relative centrifugal force $(x \mathrm{~g})$ for $10 \mathrm{~min}$. Pellets were transferred into sterile $2 \mathrm{ml}$-tubes and frozen in liquid nitrogen. Afterwards, about $500 \mu \mathrm{l}$ glass beads and $2 \mathrm{ml}$ cold extraction buffer $(8 \mathrm{M}$ $\mathrm{LiCl}, 2 \%(\mathrm{v} / \mathrm{v}) \beta$-mercaptoethanol) were added. Plant material was ground in liquid nitrogen and $2 \mathrm{ml}$ cold extraction buffer were added. Samples were vortexed and incubated overnight at $4{ }^{\circ} \mathrm{C}$. On the next day, samples were centrifuged for 4 seconds (s) to remove cell debris. Supernatants were placed into fresh $1.5 \mathrm{ml}$-tubes and centrifuged at $18000 \times \mathrm{g}$ for $30 \mathrm{~min}$ at $4{ }^{\circ} \mathrm{C}$. Supernatants were discarded and pellets were washed with cold $70 \%(\mathrm{v} / \mathrm{v})$ ethanol. Afterwards, they were dissolved in $0.5 \mathrm{ml}$ solubilization buffer ( $0.5 \%$ (w/v) sodium dodecyl sulphate (SDS), $100 \mathrm{mM} \mathrm{NaCl}, 25 \mathrm{mM}$ ethylenediaminetetraacetic acid (EDTA), $10 \mathrm{mM}$ trishydroxymethylaminomethane (Tris)- 
$\mathrm{HCl}, \mathrm{pH}$ 7.6). The aqueous phase was extracted once with an equal volume of phenol $(\mathrm{pH} 7.6)$, once in phenol/chloroform/isoamyl alcohol (25:24:1, v/v/v) and once in chloroform/isoamyl alcohol $(24: 1, \mathrm{v} / \mathrm{v})$. The aqueous phase was transferred into fresh $1.5 \mathrm{ml}-$ tubes and 0.1 volume $3 \mathrm{M} \mathrm{NaOAc}$ solution as well as 1.5 volume ethanol were added. Samples were incubated for 2 hours at $-80^{\circ} \mathrm{C}$ and then centrifuged for $30 \mathrm{~min}$ at $4{ }^{\circ} \mathrm{C}$ and maximum speed. Supernatants were discarded and $0.5 \mathrm{ml} 3 \mathrm{M} \mathrm{NaOAc}$ solution was added to the pellets. Samples were vortexed for $1 \mathrm{~min}$ and then centrifuged for 10 min at $4{ }^{\circ} \mathrm{C}$ and maximum speed. Supernatants were discarded again. Pellets were washed with $70 \%(\mathrm{v} / \mathrm{v})$ ethanol, dried at $23{ }^{\circ} \mathrm{C}$ and then dissolved in $20 \mu \mathrm{ldd} \mathrm{H}_{2} \mathrm{O}$.

\subsection{2 cDNA synthesis}

Copy deoxyribonucleic acid (cDNA) can be synthesized from messengerRNA (mRNA) by reverse transcription (Mullis and Faloona, 1987). For this method, isolated template mRNAs as well as RNA-dependent deoxyribonucleic acid (DNA) polymerase, deoxyribonucleotides (dNTPs) and oligo(dT) 18 -primers are required. Prior to the reverse transcription process, the isolated RNA was treated with DNAsel (Fermentas, St. Leon Rot, Germany) according to the manufacturer's instructions to degrade residual genomic DNA in the sample. Afterwards, RevertAid H Minus First Strand cDNA synthesis kit (Fermentas, St. Leon Rot, Germany) was used to prepare cDNA according to the given recommendations in the manual.

\subsubsection{Isolation of genomic DNA from yeast pellets and plant material}

Genomic DNA was isolated with cetyltrimethylammoniumbromid (CTAB) to verify genomic mutations in knockout yeast as well as in mutant plants. Yeast material was centrifuged at $4{ }^{\circ} \mathrm{C}$ and $3320 \times \mathrm{g}$ for $10 \mathrm{~min}$, cell pellets were transferred into $1.5 \mathrm{ml}$-tubes and frozen in liquid nitrogen. Plant tissue was pulverized in $1.5 \mathrm{ml}$-tubes using plastic pestles and liquid nitrogen. $250 \mu \mathrm{l} \mathrm{CTAB}$ solution (2\% (w/v) CTAB, $100 \mathrm{mM}$ Tris- $\mathrm{HCl}$, $\mathrm{pH}$ 8.0, $20 \mathrm{mM}$ EDTA, pH 8.0, $1.4 \mathrm{M} \mathrm{NaCl}$ ) containing $2 \%(\mathrm{v} / \mathrm{v}) \beta$-mercaptoethanol were added to the samples. These were incubated for $30 \mathrm{~min}$ to 1 hour at $65^{\circ} \mathrm{C}$. Afterwards, an equal volume of chloroform/isoamyl alcohol $(24: 1, \mathrm{v} / \mathrm{v})$ was added and samples were mixed well. Tubes were centrifuged at $7500 \times \mathrm{g}$ for $3 \mathrm{~min}$ at $23^{\circ} \mathrm{C}$. $200 \mu \mathrm{l}$ of the upper aqueous phase were transferred into fresh tubes, and 1/10 volume $\mathrm{CTAB} / \mathrm{NaCl}$ solution $\left(10 \%(\mathrm{w} / \mathrm{v}) \mathrm{CTAB}, 0.7 \%(\mathrm{w} / \mathrm{v}) \mathrm{NaCl}\right.$; preheated to $65^{\circ} \mathrm{C}$ ) was added. Samples were mixed well and incubated for $2 \mathrm{~min}$ at $23^{\circ} \mathrm{C}$. Afterwards, an equal volume of isopropanol was added, samples were mixed by inverting the tubes several times and again incubated for $2 \mathrm{~min}$ at $23^{\circ} \mathrm{C}$. Tubes were centrifuged at $20000 \times g$ and $4{ }^{\circ} \mathrm{C}$ for $10 \mathrm{~min}$. Supernatants were discarded and pellets were washed with $100 \mu \mathrm{l} 75 \%(\mathrm{v} / \mathrm{v})$ ethanol. The ethanol was removed, pellets were dried at $23^{\circ} \mathrm{C}$ and then dissolved in $70 \mu \mathrm{lddH_{2 }}$ O. 


\subsubsection{Separation of DNA and RNA in agarose gels}

DNA and RNA fragments of different sizes were separated via electrophoresis in gels prepared from 1-2 \% (w/v) agarose in TAE buffer (40 mM Tris-acetate, $\mathrm{pH}$ 8.3, $2 \mathrm{mM}$ EDTA). Prior to loading RNA samples, gel chamber, sliding carriage and gel comb were incubated for at least $20 \mathrm{~min}$ in $1 \%(\mathrm{w} / \mathrm{v})$ SDS solution to inactivate RNAses. Before loading the samples into the gel pockets they were mixed with loading buffer $(40 \%$ (v/v) glycerol, $100 \mathrm{mM}$ EDTA, pH 8.0, $0.1 \%$ (w/v) SDS, $0.25 \%$ (w/v) bromphenolblue, $0.25 \%(\mathrm{w} / \mathrm{v})$ xylencyanol) in the ratio 10:1. For length and concentration determination of the loaded DNA fragments $2.5 \mu \mathrm{g}$ GeneRuler $1 \mathrm{~kb}$ DNA Ladder (Fermentas, St. Leon Rot, Germany) were loaded into a separate gel pocket. Gels were run in TAE buffer for $30 \mathrm{~min}$ at $13 \mathrm{~V} / \mathrm{cm}$. Afterwards, gels were incubated for $15 \mathrm{~min}$ in an ethidium bromide bath $(2 \mu \mathrm{g} / \mathrm{ml}$ ethidium bromide in TAE buffer), afterwards DNA bands were visualized with the gel detection system IDA (Raytest, Straubenhardt, Germany).

\subsubsection{Polymerase chain reaction (PCR)}

PCR is used to amplify a specific DNA sequence from a given template, like genomic DNA, cDNA or plasmid DNA. Besides the template, also dNTPs, primers binding to the 3'- and 5'-ends of the selected DNA sequence as well as thermostable DNA-dependent DNA polymerase are required for this method. The PCR is separated into the three different phases of denaturation, annealing and extension. During denaturation, the DNA template is split into its two complementary strands. During annealing, primers are binding to their complementary sequences and can then be used during extension as starting points for the polymerase to synthesize the complete complementary strand. This cycle is repeated 25-35 times, resulting in an exponential amplification of the desired sequence in the reaction mixture. The PCR reactions described in this work were performed with Advantage PCR Enzyme System (Clontech, Mountain View, CA, USA), TaKaRa ExTaq DNA polymerase (Cambrex BioScience, Potsdam, Germany) or Phusion DNA polymerase (Finnzymes, Espoo, Finland). For analytical purposes $25 \mu \mathrm{l}$ reactions and for preparative purposes $50 \mu$-reactions were set up according to the manufacturer's recommendations. A typical PCR profile for Phusion DNA polymerase is given below and was used, if not stated otherwise.

$\begin{array}{lll}\text { 1. } & 98^{\circ} \mathrm{C} & 1 \mathrm{~min} \\ \text { 2. } & 98{ }^{\circ} \mathrm{C} & 30 \mathrm{~s} \\ \text { 3. } & \mathrm{T}_{\mathrm{m}}-3^{\circ} \mathrm{C} & 30 \mathrm{~s} \\ \text { 4. } & 72{ }^{\circ} \mathrm{C} & 30 \mathrm{~s} / \mathrm{kb}\end{array}$

Steps 2-4 are repeated for 30 times

5. $72{ }^{\circ} \mathrm{C} \quad 3 \mathrm{~min}$

$\mathrm{T}_{\mathrm{m}}$ is the specific melting temperature of the chosen primers and can be calculated as follows (Suggs et al., 1981):

$\mathrm{T}_{\mathrm{m}}\left[{ }^{\circ} \mathrm{C}\right]=2^{*}($ sum of $\mathrm{A}+\mathrm{T})+4^{*}($ sum of $\mathrm{G}+\mathrm{C})$ 
Primers were ordered from Sigma (Munich, Germany) or Invitrogen (Karlsruhe, Germany), primer sequences of utilized primers are listed in the appendix.

\subsubsection{Restriction digest}

Restriction enzymes cut specifically in palindromic DNA sequences, thereby either leading to sticky or blunt ends that can subsequently be ligated with ends of the same kind and thus are helpful tools for molecular cloning. Plasmid DNA or PCR-derived DNA fragments were treated with one to two restriction enzymes according to the manufacturer's instructions. This method was used to either verify the presence of a DNA fragment after plasmid DNA isolation or to prepare both insert and vector for (sub-)cloning procedures. For the former purpose a $3 \mu \mathrm{l}$-aliquot of isolated plasmid DNA was treated with restriction enzymes and then run on an agarose gel (see 2.11.4) to check for the right fragment size. For the latter purpose both vectors, the donor vector containing the DNA fragment of interest and the acceptor vector for this fragment, were cut with the same combination of restriction enzymes. The restriction digest reaction of the acceptor vector was stopped either by incubation at $85^{\circ} \mathrm{C}$ for $20 \mathrm{~min}$ or by gel-purification (see 2.11.7). In some cases, the acceptor vector was also treated with Calf Intestine Alkaline Phosphatase (Fermentas, St. Leon Rot, Germany) according to manufacturer's instructions to prevent single-cut vectors from religation later on. The insert fragment was gel-purified to remove donor vector and restriction enzymes prior to ligation.

\subsubsection{Isolation of DNA fragments from agarose gels}

DNA fragments were isolated from agarose gels by running them in the gel as described in section 2.11.4. The respective DNA band was then cut out of the gel. Afterwards, NucleoSpin Extract Kit (Macherey-Nagel, Düren, Germany) was used for eluting the DNA according to the manufacturer's protocol.

\subsubsection{Ligation}

Gel-purified PCR fragments were ligated with the TA cloning vector pGEM-T (Promega, Madison, WI, USA) according to the manufacturer's proctocol. When the preceding PCR had been performed with Phusion DNA polymerase (Finnzymes, Espoo, Finland), DNA fragments had to be treated with TaKaRa ExTaq DNA polymerase prior to ligation in order to add a poly-A overhang. In those cases, a mix containing the PCR reaction, $1 \mu \mathrm{l} 100 \mathrm{mM}$ dNTPs and $0.1 \mu \mathrm{l} \mathrm{TaKaRa} \mathrm{ExTaq} \mathrm{DNA} \mathrm{poly-}$ merase was incubated for $10 \mathrm{~min}$ at $72{ }^{\circ} \mathrm{C}$. DNA fragments after restriction digest were ligated with yeast or plant expression vectors, treated as described in section 2.11.6, in the ratio $3: 1$ using 5 units T4 DNA ligase according to the manufacturer's manual (Fermentas, St. Leon Rot, Germany). Ligation reactions were then incubated overnight at $4{ }^{\circ} \mathrm{C}$. 


\subsubsection{Gateway Cloning}

An alternative way to combine different DNA fragments without using restriction enzymes and ligation procedures is the application of clonase enzyme. This enzyme specifically recombines sequences on donor and acceptor plasmids and thereby transfers DNA fragments from one vector to the other. For cloning, Gateway LR Clonase II Enzyme Mix (Invitrogen, Karlsruhe, Germany) was used according to the manufacturer's instructions.

\subsubsection{Transformation of $E$. coli}

By the method of transformation, free DNA can be introduced into an acceptor organism, thereby changing its genetic composition. By culturing transformed bacteria, the transferred DNA construct can for example be amplified for subsequent isolation. Many bacteria have to be treated beforehand with chemicals like calcium chloride to enhance their ability to take up foreign DNA.

\subsubsection{Preparation of chemically competent $E$. coli cells}

Competent cells were prepared according to (Inoue et al., 1990). For preculture $5 \mathrm{ml}$ LB medium containing tetracycline $(10 \mathrm{mg} / \mathrm{l})$ were set up overnight with $E$. coli XL1blue cells at $37^{\circ} \mathrm{C}$ while shaking at $200 \mathrm{rpm}$. On the next day, $400 \mathrm{ml} \mathrm{LB}$ medium with tetracycline were inoculated with the whole preculture and cells were grown at $37^{\circ} \mathrm{C}$ while shaking at $200 \mathrm{rpm}$ until they reached an optical density at $600 \mathrm{~nm}\left(\mathrm{OD}_{600}\right)$ of 0.6 . Bacteria were incubated on ice for $10 \mathrm{~min}$, afterwards centrifuged for $10 \mathrm{~min}$ at $4{ }^{\circ} \mathrm{C}$ and $1000 \times \mathrm{g}$. The medium was completely removed, cells were resuspended in $120 \mathrm{ml}$ transformation buffer (TFB; $10 \mathrm{mM}$ piperazine-1,4-bis(2-ethanesulfonic acid) (Pipes), $15 \mathrm{mM} \mathrm{CaCl}_{2}, 250 \mathrm{mM} \mathrm{KCl}$ and $55 \mathrm{mM} \mathrm{MnCl}_{2}$ ) and incubated on ice for $10 \mathrm{~min}$. Then they were centrifuged at $4{ }^{\circ} \mathrm{C}$ and $1000 \times \mathrm{g}$. Buffer was removed completely, cells were resuspended in $32 \mathrm{ml}$ TFB buffer containing $7 \%(\mathrm{v} / \mathrm{v})$ DMSO and incubated again for $10 \mathrm{~min}$ on ice. Afterwards, they were aliquoted into units of 200-500 $\mu$, frozen in liquid nitrogen and stored at $-80^{\circ} \mathrm{C}$ until use.

\subsubsection{Transformation of competent E. coli cells}

For transformation, competent cells were thawn gently on ice. Then, $100 \mu \mathrm{l}$ of cells were added either to $10 \mu \mathrm{l}$ ligation reaction, $5 \mu \mathrm{l}$ clonase reaction mix or $1 \mu \mathrm{l}$ plasmid DNA and incubated on ice for $20 \mathrm{~min}$. Cells were put to $42{ }^{\circ} \mathrm{C}$ for $35 \mathrm{~s}$ and then immediately placed on ice again. $600 \mu \mathrm{LB}$ medium were added to the cells and they were incubated for one hour at $37^{\circ} \mathrm{C}$ while shaking at $200 \mathrm{rpm}$. Then, the appropriate amount of cells was plated on solid LB medium containing the respective antibiotic as selection marker. Plates were incubated overnight at $37^{\circ} \mathrm{C}$. 


\subsubsection{Isolation of plasmid DNA from E. coli}

For plasmid DNA isolation, positive clones were chosen after transformation and inoculated overnight at $37^{\circ} \mathrm{C}$ while shaking at $200 \mathrm{rpm}$ in $2 \mathrm{ml}$ liquid LB medium containing the respective antibiotic as selection marker. NucleoSpin Plasmid Kit from MachereyNagel (Düren, Germany) was then used according to the manufacturer's instructions.

\subsubsection{Sequencing}

Sequencing is crucial to prove the accuracy of PCR-amplified sequences in plasmids. The method modified from (Sanger et al., 1977) is based on intermixed dideoxynucleotides (ddNTPs) in the PCR reaction which lead to abortion of chain elongation. They are conjugated with base-specific fluorescent dyes, thereby the sequence of the analyzed DNA fragment can be read out. For sequencing, ABI Prism BigDye Terminator (BDT) Cycle Sequencing Ready Reaction Kit v1.1 (Applied Biosystems, Foster, USA) and sequence- as well as vector-specific primers were used. 200-300 ng plasmid DNA as template, $1.5 \mu \mathrm{l}$ BDT Ready Reaction Mix, $1.5 \mu$ half term buffer, $1 \mu \mathrm{l}$ sequencing primer $(0.5 \mu \mathrm{M})$ and $5 \mu \mathrm{l}$ sterile $\mathrm{ddH}_{2} \mathrm{O}$ were mixed in PCR tubes. PCR parameters were initial denaturation for $1 \mathrm{~min}$ at $96{ }^{\circ} \mathrm{C}$ followed by 25 cycles $96{ }^{\circ} \mathrm{C}$ denaturation for $5 \mathrm{~s}, 55^{\circ} \mathrm{C}$ annealing for $15 \mathrm{~s}$ and $60^{\circ} \mathrm{C}$ extension for $4 \mathrm{~min}$. After PCR, reactions were transferred into fresh $1.5 \mathrm{ml}$-tubes and DNA was precipitated by mixing samples with $1 \mu \mathrm{l} 3 \mathrm{M} \mathrm{NaOAc}(\mathrm{pH} 5), 1 \mu \mathrm{l} 125 \mathrm{mM}$ EDTA and $25 \mu \mathrm{l} 96 \%$ (v/v) ethanol. Samples were incubated for $15 \mathrm{~min}$ at $23^{\circ} \mathrm{C}$ and then centrifuged at maximum speed for $15 \mathrm{~min}$ in a microcentrifuge. Supernatants were discarded and DNA pellets were washed with $50 \mu \mathrm{l} 70 \%(\mathrm{v} / \mathrm{v})$ ethanol. Supernatants were discarded and pellets were dried for $1 \mathrm{~min}$ at $90{ }^{\circ} \mathrm{C}$. Then they were resuspended in $15 \mu \mathrm{l}$ formamide and stored at $-20{ }^{\circ} \mathrm{C}$ until analysis. This was performed with ABI PRISM 3100 Genetic Analyser (Applied Biosystems, Foster, USA). Evaluation of the obtained data was done using the program Chromas lite (www.technelysium.com.au) as well as the online softwares MultAlin (http://bioinfo.genopole-toulouse.prd.fr/multalin/multalin.html, according to (Corpet, 1988) and Boxshade 3.21 (http://www.ch.embnet.org/software/BOX_form.html).

\subsection{Isolation and cloning of studied cDNA sequences}

\subsubsection{Cloning of microalgal acyltransferase sequences into yeast expression vectors}

OILPCAT from O. Iucimarinus was ordered as completely codon-optimized version by Dr. M. Wagner. OtLPCAT had been isolated from $O$. tauri and partly codon-optimized by Dr. M. Heilmann. Templates for the conducted PCRs are listed in section 2.6. OILPCAT was amplified using primers OILPCATcoforBamHI and OILPCATcorevApal (all primers are listed in the appendix). OtLPCAT was amplified using primers OtLPCATtcoforBamHI and OtLPCATtcorevNhel. Resulting DNA fragments containing a translation initiation signal were treated with the respective restriction enzymes, gel- 
purified and ligated into pESC-LEU cut with the same combinations of enzymes. OtLPCAT in addition was also cloned into pYES2/CT. For creating a nucleotide sequence without stop codon, primer combination OtLPCATtcoforBamHI and OtLPCATtcorev-StopXbal was used. In order to obtain a PCR-derived sequence with stop codon, primers OtLPCATtcoforBamHI and OtLPCATtcorevNotl were utilized. Resulting DNA fragments containing a translation initiation signal were treated with the respective restriction enzymes, gel-purified and ligated into pYES2/CT cut with the same combinations of enzymes. Positive clones were sequenced and then used for transformation of yeast.

To generate GFP-fusion constructs, appropriate restriction sites were added by PCR using primers OILPCATcoforHindIII and OILPCATcorevXhol or OtLPCATtcoforSpel and OtLPCATtcorevHindIII, respectively. The resulting PCR products were gel-purified, ligated into pUG36 cut with the same combinations of enzymes and sequenced prior to transformation into yeast.

OtDGAT2A and OtDGAT2C were ordered as completely codon-optimized versions by Dr. M. Wagner. Templates for the conducted PCRs are listed in section 2.6. Ot$D G A T 2 A$ and OtDGAT2C were amplified using primers OtDGAT2AcoforEcoRI and OtDGAT2AcorevNotl or OtDGAT2CcoforEcoRI and OtDGAT2CcorevNotl, respectively. Resulting DNA fragments containing a translation initiation signal were treated with the corresponding restriction enzymes, gel-purified and ligated into mCherryFAR1-pESCURA (provided by Dr. M. Heilmann) cut with the same combination of enzymes. Positive clones were sequenced and then used for transformation of yeast.

\subsubsection{Isolation, codon-optimization and cloning of Old4 from 0 . lucimarinus into different yeast expression vectors}

Old4 was isolated from genomic DNA (provided by Dr. M. Heilmann) using primers Old4for, Old4hppgfor, Old4mfor, Old4hppgrev, Old4mrev and Old4rev. The front and middle fragments were amplified by PCR using the Advantage PCR Enzyme System and primer combinations Old4for-Old4hppgrev and Old4hppgfor-Old4mrev, respectively. PCR parameters were initial denaturation for $3 \mathrm{~min}$ at $95{ }^{\circ} \mathrm{C}$ followed by 25 cycles $95^{\circ} \mathrm{C}$ denaturation for $30 \mathrm{~s}, 70{ }^{\circ} \mathrm{C}$ annealing for $30 \mathrm{~s}$ and $72{ }^{\circ} \mathrm{C}$ extension for $90 \mathrm{~s}$. In the end, a final $72{ }^{\circ} \mathrm{C}$ extension phase of 5 min was added. The rear fragment of the gene was amplified with Phusion DNA polymerase. The primers used were Old4mfor and Old4rev. PCR parameters were initial denaturation for 3 min at $98^{\circ} \mathrm{C}$ followed by 25 cycles $98^{\circ} \mathrm{C}$ denaturation for $30 \mathrm{~s}, 60^{\circ} \mathrm{C}$ annealing for $30 \mathrm{~s}$ and $72{ }^{\circ} \mathrm{C}$ extension for $70 \mathrm{~s}$. In the end, a final $72{ }^{\circ} \mathrm{C}$ extension phase of 5 min was added. Afterwards, an overlap extension PCR with Phusion DNA polymerase using primers Old4for and Old4rev and the three different fragments as template was performed. PCR parameters were initial denaturation for 2 min at $98^{\circ} \mathrm{C}$ followed by 10 cycles $98^{\circ} \mathrm{C}$ denaturation for $15 \mathrm{~s}, 68^{\circ} \mathrm{C}$ annealing for $30 \mathrm{~s}$ and $72{ }^{\circ} \mathrm{C}$ extension for $60 \mathrm{~s}$. Then, 20 cycles of $98^{\circ} \mathrm{C}$ denaturation for $15 \mathrm{~s}, 70{ }^{\circ} \mathrm{C}$ annealing for $30 \mathrm{~s}$ and $72{ }^{\circ} \mathrm{C}$ extension for $60 \mathrm{~s}$ followed. In the end a final $72{ }^{\circ} \mathrm{C}$ extension phase of $7 \mathrm{~min}$ was added. 
The resulting DNA fragment was gel-purified and cloned into pGEM-T for sequencing. Phusion DNA polymerase was used for the following PCR reactions using a positive clone as template. Codon-optimization of the first 20 codons for the expression in yeast was performed using primers Old4tcofor and Old4rev. In a second reaction, restriction sites and an ACATA nucleotide sequence as translation initiation signal in front of the start codon (Donahue and Cigan, 1990) were introduced into the sequence using primers Old4tcoforEcoRI and Old4revNotl. PCR parameters were initial denaturation for $1 \mathrm{~min}$ at $98{ }^{\circ} \mathrm{C}$ followed by 25 cycles $98{ }^{\circ} \mathrm{C}$ denaturation for $30 \mathrm{~s}, 50-60{ }^{\circ} \mathrm{C}$ annealing for $1 \mathrm{~min}$ and $72{ }^{\circ} \mathrm{C}$ extension for $70 \mathrm{~s}$. In the end, a final $72{ }^{\circ} \mathrm{C}$ extension phase of 5 min was added. All PCR reactions of this type were performed in the Mastercycler gradient Eppendorf AG (Hamburg, Germany). The resulting PCR product was cloned into the EcoRI and Notl sites of the vector pESC-TRP under the control of the inducible promoter GAL10. The resulting construct was sequenced before transformation into yeast. To generate a GFP-fusion construct, the prepared pESC-TRP construct was used as template for amplification and addition of appropriate restriction sites by PCR using primers Old4tcoforEcoRl and Old4revXhol. The resulting PCR product was gelpurified, ligated into the EcoRl and Xhol sites of pUG36 and sequenced prior to transformation into yeast.

\subsubsection{Cloning of Egd4 from E. gracilis into different yeast expression vectors}

The construct Ms $\Delta 6$-PSE1-Ms $\Delta 5-O t E L O 5-E g d 4-p C A M B I A 3300$ was provided by $\mathrm{S}$. Schlenczek. The open reading frame of Egd4 was amplified using primers Egd4forBamHI and Egd4revXhol in a "touch-down" PCR using Phusion DNA polymerase. PCR parameters were initial denaturation for $2 \mathrm{~min}$ at $98{ }^{\circ} \mathrm{C}$ followed by 20 cycles $98{ }^{\circ} \mathrm{C}$ denaturation for $20 \mathrm{~s}, 72{ }^{\circ} \mathrm{C}$ annealing for $30 \mathrm{~s}$ and $72{ }^{\circ} \mathrm{C}$ extension for $70 \mathrm{~s}$. During each cycle, the annealing temperature was lowered $0.1{ }^{\circ} \mathrm{C} / \mathrm{s}$. Then, 20 cycles $98{ }^{\circ} \mathrm{C}$ denaturation for $20 \mathrm{~s}, 70{ }^{\circ} \mathrm{C}$ annealing for $30 \mathrm{~s}$ and $72{ }^{\circ} \mathrm{C}$ extension for $70 \mathrm{~s}$ followed. During each cycle, the annealing temperature was lowered $0.3^{\circ} \mathrm{C} / \mathrm{s}$. The resulting DNA fragment was sublconed into pGEM-T. A positive clone was subjected to sequencing and then used for restriction digest with $\mathrm{BamHI}$ and Xhol. The resulting fragment was cloned into the $\mathrm{BamHI}$ and $\mathrm{Xhol}$ sites of $\mathrm{pESC}$-TRP. To generate a GFP. fusion construct, the prepared pESC-TRP construct was used as template for amplification and addition of appropriate restriction sites by PCR using primers Egd4forEcoRI and Egd4revXhol. The resulting PCR product was cloned into the EcoRI and Xhol sites of pUG36 and sequenced prior to transformation into yeast. 


\subsubsection{Isolation and cloning of TpLACS from $T$. pseudonana into a yeast expression vector}

The long-chain acyl-CoA synthetase nucleotide sequence from $T$. pseudonana (Tonon et al., 2005a) was isolated via PCR from cDNA prepared from total RNA using primers TpLACSfor and TpLACSrev. The resulting PCR product was used as template in a second $\mathrm{PCR}$ reaction to add restriction sites and a translation initiation signal with primers TpLACSforApal and TpLACSrevNhel. The resulting DNA fragment was subcloned into pGEM-T. A positive clone was subjected to sequencing and then used for restriction digest with Apal and Nhel. The resulting fragment was cloned into the Apal and Nhel sites of $\mathrm{pESC}$-LEU.

\subsubsection{Isolation and cloning of CsMGDGS from Cucumis sativus into a yeast expression vector}

The monogalactosyldiacylglycerol synthase nucleotide sequence from $C$. sativus (Shimojima et al., 1997) was amplified using a cDNA bank prepared from four days old etiolated cucumber seedlings (provided by Dr. E. Hornung) as template. The sequence was amplified without its chloroplastidial targeting signal utilizing primers CsMGDGSforBamHI and CsMGDGSrevNhel in a "touch-down" PCR as described in section 2.12.3. The resulting PCR product containing a translation initiation signal was cloned into the BamHI and Nhel sites of pESC-LEU.

\subsubsection{Cloning of microalgal and plant acyltransferase sequences into plant expression vectors}

OtDGAT2B, OILPCAT and OtPDAT were isolated by Dr. M. Wagner from O. tauri and $O$. lucimarinus, respectively. OtLPCAT was isolated from 0 . tauri and AtDGAT1 as well as AtDGAT2 were isolated from $A$. thaliana by $\mathrm{Dr}$. M. Heilmann. Templates for the conducted PCRs are listed in section 2.6. OtDGAT2B was amplified using primers OtDGAT2BtcoforBamHI and OtDGAT2BtcorevNotI. OILPCAT was amplified using primers OILPCATcoforBamHI and OILPCATcorevNotI. OtPDAT was amplified using primers OtPDATtcoforEcoRI and OtPDATtcorevNotI. OtLPCAT was amplified using primers OtLPCATtcoforBamHI and OtLPCATtcorevNotI. AtDGAT1 was amplified using primers AtDGAT1forKpnl and AtDGAT1revXhol. AtDGAT2 was amplified from the template using primers AtDGAT2forEcoRI and AtDGAT2revXhol. For all DNA fragments, "touchdown" PCRs like described in section 2.12.3 were performed. Afterwards, they were treated with the respective restriction enzymes and ligated into the pUC18-Entry2 cut with the same combinations of enzymes. Positive clones were sequenced and subjected to clonase reaction (see section 2.11.9) with pCAMBIA33.2cGs. Clonase reaction mixtures were used for $E$. coli transformation. 


\subsubsection{Cloning of Msd6 and OILPCAT into Ptd6-PSE1-Ptd5-pCAMBIA3300}

Ptd6-PSE1-Ptd5 and Msd6 or Msd6-OILPCAT or OILPCAT, respectively, were inserted sequentially into pCAMBIA3300 by first digesting the destination vector with Sbfl and ligating it with the expression cassette cut out from Ptd6-PSE1-Ptd5-pUC19-USPOCS1 with Sbfl. A positive clone was then cleaved with Ascl and ligated with the expression cassettes cut out from Msd6-pUC19-USP-OCS123 or Msd6-OILPCATpUC19-USP-OCS123 or OILPCAT-pUC19-USP-OCS123 with Ascl. Positive clones were used for transformation of $A$. tumefaciens.

\subsection{Genetic engineering methods}

\subsubsection{Transformation of $A$. tumefaciens}

A. tumefaciens cells were transformed with plasmid DNA isolated from $E$. coli in order to use the positive transformants for subsequent $A$. tumefaciens-mediated transformation of $A$. thaliana or $C$. sativa plants.

\subsubsection{Preparation of chemically competent $A$. tumefaciens cells}

For preparation of chemically competent cells, the method developed by (Höfgen and Willmitzer, 1988) was applied. For preculture, $2 \mathrm{ml}$ YEB medium containing rifampicin (50 mg/l) were set up overnight with $A$. tumefaciens cells at $28{ }^{\circ} \mathrm{C}$ while shaking at $200 \mathrm{rpm}$. On the next day, $50 \mathrm{ml}$ YEB medium were inoculated with the whole preculture and cells were grown at $28{ }^{\circ} \mathrm{C}$ while shaking at $200 \mathrm{rpm}$ for 4 hours until they reached an $\mathrm{OD}_{600}$ of 0.5 . Bacteria were centrifuged at $4{ }^{\circ} \mathrm{C}$ and $2300 \times \mathrm{g}$ for $5 \mathrm{~min}$. The medium was completely removed and cells were resuspended in $10 \mathrm{ml} 0.15 \mathrm{M}$ $\mathrm{NaCl}$ solution. Then they were centrifuged again, the buffer was completely removed and cells were resuspended in $1 \mathrm{ml}$ ice cold $75 \mathrm{mM} \mathrm{CaCl}$ solution. Afterwards, $200 \mu \mathrm{l}-$ aliquots were taken, frozen in liquid nitrogen and stored at $-80^{\circ} \mathrm{C}$ until use.

\subsubsection{Transformation of competent $A$. tumefaciens cells}

For transformation, $200 \mu$ l-aliquots with competent cells were thawn at $37{ }^{\circ} \mathrm{C}$. Then, $3 \mu \mathrm{g}$ plasmid DNA were added and cells were incubated at least for $30 \mathrm{~min}$ on ice. Once in a while they were mixed gently. Afterwards, the mix was frozen for $2 \mathrm{~min}$ at $-80{ }^{\circ} \mathrm{C}$ and immediately thawn again at $37^{\circ} \mathrm{C}$. $800 \mu$ YEB medium were added to the cells and they were incubated for $1-4$ hours at $28{ }^{\circ} \mathrm{C}$ while shaking at $200 \mathrm{rpm}$. Then, cells were sedimented, plated on solid YEB medium containing the respective antibiotic as selection marker and incubated for 2 days at $28^{\circ} \mathrm{C}$. 


\subsubsection{Transformation of S. cerevisiae}

S. cerevisiae cells were transformed according to (Ito et al., 1983) with plasmid DNA isolated from $E$. coli or with linear fragments produced by PCR. Plasmid DNA was transformed in order to use the positive yeast transformants for subsequent biochemical analysis of the encoded enzymes. Selection was based on the auxotrophy of the utilized yeast strains for several amino acids. The auxotrophic marker was combined with the cDNA of interest on a yeast vector. Alternatively, linear fragments were transformed to obtain knockout mutants. Then, selection was based on the acquired resistance of the knockout yeast towards hygromycin B as described in section 2.13.3.

As preculture, $2 \mathrm{ml}$ YPD medium were inoculated overnight with the respective yeast strain and shaken at $30^{\circ} \mathrm{C}$ and $200 \mathrm{rpm}$. On the next day, $5 \mathrm{ml}$ YPD medium per transformation reaction were inoculated with the appropriate amount of preculture to have a starting $\mathrm{OD}_{600}$ of 0.2 . Cells were grown at $30{ }^{\circ} \mathrm{C}$ while shaking at $200 \mathrm{rpm}$ until their $\mathrm{OD}_{600}$ reached 0.8 . Cells were centrifuged at $1600 \times \mathrm{g}$ for $3 \mathrm{~min}$ at $23^{\circ} \mathrm{C}$, the supernatant was discarded and cells were washed once with $5 \mathrm{ml}$ sterile TE buffer $(10 \mathrm{mM}$ Tris-HCl, $1 \mathrm{mM}$ EDTA, pH 8). Cells were resuspended in $100 \mu \mathrm{l} 0.1 \mathrm{M}$ lithium acetate in TE buffer and incubated for $10 \mathrm{~min}$ at $23{ }^{\circ} \mathrm{C}$. The suspension was mixed with either $5 \mu \mathrm{l}$ plasmid DNA or $50 \mu \mathrm{l}$ purified PCR product combined with $10-25 \mu \mathrm{l}$ denatured single stranded herring sperm carrier DNA and $700 \mu$ transformation mix (40\% (w/v) polyethylene glycol (PEG) 4000, $0.1 \mathrm{M}$ lithium acetate, $10 \mathrm{mM}$ Tris-HCl, $1 \mathrm{mM}$ EDTA, $\mathrm{pH}$ 8). Suspensions were incubated for $30 \mathrm{~min}$ at $30{ }^{\circ} \mathrm{C}$ while shaking at $200 \mathrm{rpm}$. Afterwards, they were shifted to $42^{\circ} \mathrm{C}$ for $15 \mathrm{~min}$ and centrifuged at $4500 \times \mathrm{g}$ for one minute. Supernatants were removed, cells transformed with plasmid DNA were washed in $500 \mu \mathrm{l} \mathrm{TE}$ buffer and then resuspended in $50 \mu \mathrm{l} \mathrm{TE}$ buffer. Afterwards, they were plated on solid SD medium containing $2 \%(\mathrm{w} / \mathrm{v})$ glucose and the respective $50 \mathrm{x}$ synthetic complete drop-out medium mix for selection of the transformants. In case of transformation with linear DNA for gene deletion, cells were resuspended in $1 \mathrm{ml}$ YPD medium and incubated for another $2-3$ hours at $30{ }^{\circ} \mathrm{C}$ while shaking at $200 \mathrm{rpm}$. Then, cells were sedimented and plated on solid YPD medium containing hygromycin $B$ for selection purposes.

\subsubsection{PCR-based gene deletion in S. cerevisiae}

In order to obtain different yeast knockout strains, PCR-based gene deletion according to (Wach et al., 1994; Guldener et al., 1996; Goldstein and McCusker, 1999) was applied. This technique is based on the fact that homologous recombination with linear DNA fragments occurs very easily in yeast. Linear DNA fragments for knockout creation were prepared by fusing a hygromycin B phosphotransferase gene containing hphMX4-resistance cassette with flanking 3'- and 5'-sequences of the gene that had to be removed in a PCR reaction. As template for the PCR, plasmid DNA containing the resistance cassette was used, and for every gene specific primers were designed (pro- 
vided by Dr. Martin Fulda and Dr. Michael Scharnewski). The PCR product was purified using NucleoSpin Extract Kit (Macherey-Nagel, Düren, Germany) and transformed into yeast (see 2.13.2). During transformation, the gene of interest was then replaced by homologous recombination with the introduced PCR product. Positive transformants were selected on YPD medium containing hygromycin B. Genomic DNA was isolated from these clones (see 2.11.3) and used as template for control PCRs checking for successful knockout of the gene of interest. For these PCRs, gene specific as well as resistance cassette specific primers were used.

\subsubsection{Transformation of plants}

Flourishing $A$. thaliana and $C$. sativa plants were transformed by the flower-dipping method developed by (Clough and Bent, 1998) using $A$. tumefaciens EHA105 cells as mediating vector. Transgenic T1 plants were then selected by kanamycin and/or Basta in order to obtain T2 transgenic seeds for biochemical analyses or sowing to gain T3 seeds.

\subsubsection{Transformation of $A$. thaliana}

Transformed $A$. tumefaciens cells were cultivated in $400 \mathrm{ml}$ YEB medium containing the required selection antibiotics at $28{ }^{\circ} \mathrm{C}$ while shaking at $200 \mathrm{rpm}$ until they reached an $\mathrm{OD}_{600}$ of 2. Afterwards, cells were centrifuged at $4{ }^{\circ} \mathrm{C}$ and $2000 \times \mathrm{g}$ for $10 \mathrm{~min}$ and resuspended in $200 \mathrm{ml} 5 \%$ (w/v) sucrose-solution. Suspensions were incubated for 20 min on ice, afterwards $100 \mu \mathrm{l} 0.05 \%$ (v/v) Silwet L-77 (OSI Specialties, Danbury, CT, USA) were added to reduce the surface tension of the flowers. The influorescences of Arabidopsis plants were dipped for approximately $5 \mathrm{~s}$ into the bacterial solution. After dipping, plants were kept for at least 4 hours out of direct light and under a plastic cover in order to assure high air humidity, before they were put back to the greenhouse.

\subsubsection{Transformation of C. sativa}

The method applied was similar to the one established by (Lu and Kang, 2008) and included vacuum infiltration. Bacterial cells were prepared as described above (see 2.13.4.1). After incubation on ice, the beaker with the cell suspension was placed in an exsiccator. Up to six Camelina-plants were positioned around the beaker, their influorescences were dipped into the bacterial solution. The exsiccator was closed and a vacuum of $-40 \mathrm{kPa}$ was generated. It was held for $5 \mathrm{~min}$, then air was allowed to stream into the exsiccator in a slow and controlled way. After dipping, plants were also kept for at least 4 hours out of direct light and under a plastic cover, before they were put back to the greenhouse. 


\subsection{Heterologous expression of cDNAs in S. cerevisiae}

For functional characterization of enzymes, the respective cDNAs were expressed in different yeast strains that were auxotrophic for several amino acids. This feature was used for selection of transgenic cells containing the desired cDNA sequence combined with an auxotrophic marker on a yeast vector. In vectors containing the GAL1 and/or the GAL10 promoter like pESC- and pYES-vectors, expression was induced by galactose. Expression using the vector pUG36 was induced by absence of methionine.

As precultures, $2-10 \mathrm{ml} \mathrm{SD}$ medium containing $2 \%(\mathrm{w} / \mathrm{v})$ raffinose and the respective synthetic dropout mix for selection were inoculated with transformed yeast colonies. Cultures were grown overnight at $30^{\circ} \mathrm{C}$ while shaking at $200 \mathrm{rpm}$. On the next day $10-$ $100 \mathrm{ml} \mathrm{SD}$ medium containing $2 \%$ (w/v) galactose for expression induction and the respective amino acid dropout mix were inoculated with the appropriate amount of preculture to have a starting $\mathrm{OD}_{600}$ of 0.3 . In case substrate specificity of enzymes should be investigated, also $1 \%$ (v/v) Igepal CA 630 (Fluka, Steinheim, Deutschland) and $200 \mu \mathrm{M}$ of free fatty acid were added. Igepal is a detergent and permeabilizes cell membranes to enhance the uptake of very long chain polyunsaturated and thus unpolar fatty acids. For protein expression tests, cultures were grown for 20-48 hours at $23^{\circ} \mathrm{C}$ while shaking at $150 \mathrm{rpm}$. In case of methionine-dependent expression, cultures were cultivated either in presence of $0.3 \mathrm{mM}$ methionine (moderate expression) or in complete absence of methionine (over-expression). For protein activity tests with or without substrates, cultures were grown for 24-96 hours at temperatures between 16$30{ }^{\circ} \mathrm{C}$ while shaking at $150-200 \mathrm{rpm}$, as indicated for each experiment. Afterwards, $\mathrm{OD}_{600}$ of the cultures was determined and cells were harvested by centrifugation for $5 \mathrm{~min}$ at $1500 \times \mathrm{g}$. Supernatants were discarded and cells were washed twice with $5 \mathrm{ml}$ sterile $\mathrm{ddH}_{2} \mathrm{O}$. The resulting cell pellet was then used for protein or lipid analyses.

\subsection{Microscopic procedures}

Yeast cells expressing GFP-Old4-pUG36 were grown in $2 \mathrm{ml}$-cultures at $30{ }^{\circ} \mathrm{C}$ to stationary phase while shaking at $200 \mathrm{rpm}$. For staining of nuclei, cells were incubated for 15 min with the dye Hoechst 33342 trihydrochloride (Molecular Probes, Invitrogen, Karlsruhe, Germany). Fluorescence and light microscopy was done using a Zeiss Axioscope 2 microscope and an Axiocam digital camera. Pictures are pseudo-colorized. 


\subsection{Protein expression analysis}

\subsubsection{Protein sample preparation}

Yeast cultures for recombinant protein expression tests were grown as described above. The sample preparation was then performed according to pYES2/CT manufacturer's recommendations (Invitrogen, Carlsbad, CA, USA). Cell pellets from $20 \mathrm{ml}-$ cultures were resuspended in $500 \mu \mathrm{l}$ sterile $\mathrm{ddH}_{2} \mathrm{O}$ and transferred to sterile $2 \mathrm{ml}$-tubes. Samples were centrifuged for $30 \mathrm{~s}$ at maximum speed, supernatants were removed and pellets were either stored at $-80^{\circ} \mathrm{C}$ or directly used to prepare cell lysates. For this purpose, cells were resuspended in $500 \mu$ of breaking buffer $(50 \mathrm{mM}$ sodium phosphate, $\mathrm{pH} 7.4,1 \mathrm{mM}$ EDTA, $5 \%$ ( $/ \mathrm{v}$ ) glycerol, $1 \mathrm{mM}$ phenylmethanesulphonylfluoride (PMSF)) and centrifuged at $1500 \times \mathrm{g}$ for $5 \mathrm{~min}$ at $4{ }^{\circ} \mathrm{C}$. Supernatants were removed and cells were resuspended in $200 \mu \mathrm{l}$ breaking buffer. An equal volume of glass beads (1.7-2 mm; Carl Roth \& Co., Karlsruhe, Germany) was added and samples were vortexed eight times for $30 \mathrm{~s}$, each time followed by $30 \mathrm{~s}$ on ice. Then they were centrifuged for $10 \mathrm{~min}$ at maximum speed in a microcentrifuge, supernatants were transferred to fresh tubes and the lysates were assayed for protein concentration using Bradford solution $(0.007 \%$ (w/v) Coomassie Brilliant Blue G-250, $4.8 \%$ (v/v) ethanol, $8.5 \%(\mathrm{v} / \mathrm{v})$ phosphoric acid) and bovine serum albumin (BSA) as a standard according to (Bradford, 1976). The dye is binding the proteins in the sample and thereby changes its colour which can be measured with a spectrophotometer. For determination of protein concentration $2 \mathrm{ml}$ Bradford reagent were mixed with $2 \mu \mathrm{l}$ of sample and $98 \mu \mathrm{l}$ $\mathrm{ddH}_{2} \mathrm{O}$. The mixture was incubated for $5 \mathrm{~min}$ at $23^{\circ} \mathrm{C}$ and then the absorption was measured at $\lambda=595 \mathrm{~nm}$.

\subsubsection{SDS-Polyacrylamide Gel Electrophoresis (PAGE)}

SDS-PAGE was performed to separate proteins of different molecular weights on the basis of (Laemmli, 1970) using Mini-PROTEAN 3 System (Biorad, Hercules, CA, USA), a $5 \%$ stacking gel and a $12 \%$ separation gel according to manufacturer's instructions. For protein size prediction, $5 \mu \mathrm{l}$ of Prestained Protein Molecular Weight Marker (Fermentas, St. Leon Rot, Germany) were loaded. 5 x SDS-PAGE sample buffer (0.225 M Tris- $\mathrm{HCl}, \mathrm{pH}$ 6.8, 50 \% (v/v) glycerol, $5 \%$ (w/v) SDS, $0.05 \%(\mathrm{w} / \mathrm{v})$ bromphenolblue, $0.25 \mathrm{M}$ dithiothreitol) was added to a final concentration of $1 \mathrm{x}$ in order to introduce an excess of negative charge into the proteins contained in the samples. Then, samples were boiled for $5 \mathrm{~min}$ to denature the proteins. $20 \mu \mathrm{g}$ of lysate were then used for SDSPAGE electrophoresis. Protein gels were run at $200 \mathrm{~V}$ in running buffer (25 mM Tris$\mathrm{HCl}, \mathrm{pH}$ 8.9, $0.2 \mathrm{M}$ glycine, $0.1 \%(\mathrm{w} / \mathrm{v}) \mathrm{SDS}$ ). After the run, gels were either stained with Coomassie Brilliant Blue G-250 or used for Western Blotting. 


\section{Stacking gel (5\%)}

$59 \%(\mathrm{v} / \mathrm{v}) \quad \mathrm{ddH}_{2} \mathrm{O}$

$23 \%(\mathrm{v} / \mathrm{v}) \quad$ Gel buffer (3 M Tris-HCl, $\mathrm{pH} 8.45,0.3 \%$ (w/v) SDS)

$18 \%(\mathrm{v} / \mathrm{v}) \quad$ Acrylamide solution (30\%, containing $0.8 \%$ bisacrylamide)

$0.4 \%(\mathrm{v} / \mathrm{v}) \quad$ Tetramethylethylenediamine (TEMED)

$0.04 \%(\mathrm{w} / \mathrm{v}) \quad$ Ammonium persulfate (APS)

\section{Separation gel (12\%)}

$40 \%(\mathrm{v} / \mathrm{v}) \quad$ Acrylamide solution (30\%, containing $0.8 \%$ bisacrylamide)

$33 \%(\mathrm{v} / \mathrm{v}) \quad$ Gel buffer (3 M Tris- $\mathrm{HCl}, \mathrm{pH} 8.45,0.3 \%$ (w/v) SDS)

$27 \%(v / v) \quad \mathrm{ddH}_{2} \mathrm{O}$

$0.1 \%(\mathrm{v} / \mathrm{v}) \quad$ TEMED

$0.05 \%(w / v) \quad$ APS

\subsubsection{Coomassie staining}

For visualization of proteins, gels were stained for $30 \mathrm{~min}$ in Coomassie staining solution (40\% (v/v) methanol, $10 \%(\mathrm{v} / \mathrm{v})$ acetic acid, $0.25 \%(\mathrm{w} / \mathrm{v})$ Coomassie Brilliant Blue G-250) on the basis of (Meyer and Lamberts, 1965). Acetic acid fixes the proteins in the gel, the dye binds basic and aromatic amino acids and thereby unspecifically stains all proteins in the gel. Unbound dye was removed by shaking gels in $\mathrm{ddH}_{2} \mathrm{O}$ for several hours.

\subsubsection{Western Blot analysis and immunodetection}

For immunodetection of epitope-tagged proteins, these were transferred from the SDSPAGE gel to nitrocellulose membrane (Macherey\&Nagel, Düren, Germany) using Mini Trans-Blot cell equipment (Bio-Rad, Hercules, CA, USA). Transfer was performed for $90 \mathrm{~min}$ at $60 \mathrm{~V}$ in the presence of prechilled transfer buffer $(0.05 \mathrm{M}$ Tris- $\mathrm{HCl}, 0.04 \mathrm{M}$ glycine, $20 \%(\mathrm{v} / \mathrm{v})$ methanol, $0.4 \%(\mathrm{w} / \mathrm{v}) \mathrm{SDS})$. Following transfer, the nitrocellulose membrane was washed in TBST buffer $(20 \mathrm{mM}$ Tris- $\mathrm{HCl}, \mathrm{pH} 7.5,50 \mathrm{mM} \mathrm{NaCl}, 0.1 \%$ $(\mathrm{v} / \mathrm{v})$ Tween 20) and incubated with blocking solution (5\% (w/v) BSA in TBST buffer) overnight at $4{ }^{\circ} \mathrm{C}$ while shaking on a rocking table. This procedure was performed to minimize unspecific binding of the antibodies. On the next day, the membrane was washed three times for 15 min while shaking in TBST buffer to remove the blocking solution. In order to detect recombinant proteins, the membrane was incubated for 216 hours at $23^{\circ} \mathrm{C}$ with the primary antibody diluted according to the manufacturer's instructions in TBST buffer containing $5 \%$ (w/v) BSA on the rocking table. After washing the membrane three times with TBST buffer for $15 \mathrm{~min}$ the secondary antibody di- 
luted in TBST buffer according to the manufacturer's instructions was added and incubated for 1 hour at $23{ }^{\circ} \mathrm{C}$ while shaking. The membrane was washed three times for 15 min in TBST buffer and the recombinant proteins were visualized using ECL Western blotting detection reagents and analysis system (GE Healthcare, Freiburg, Germany) according to manufacturer's instructions with high performance chemiluminescence film (GE Healthcare, Freiburg, Germany) and Optimax Typ TR developer (MS Labware, Wiesloch bei Heidelberg, Germany).

\subsection{Protein Activity Assays}

\subsubsection{DGAT and MGAT activity assay}

DGAT and MGAT activity assays were performed on the basis of (Czabany et al., 2008). Cell pellets of $50 \mathrm{ml}$ expression cultures were resuspended in $1 \mathrm{ml}$ DGAT assay buffer (150 mM Tris- $\mathrm{HCl}, \mathrm{pH} 7.0 ; 15 \mathrm{mM} \mathrm{KCl} ; 15 \mathrm{mM} \mathrm{MgCl}_{2}$ ) and transferred to $2 \mathrm{ml}$ tubes. Samples were centrifuged for $1 \mathrm{~min}$ at $4{ }^{\circ} \mathrm{C}$ and maximum speed in a microcentrifuge. Supernatants were discarded and pellets were resuspended in $400 \mu$ LGAT assay buffer. An equal amount of glass beads was added and samples were vortexed eight times for $30 \mathrm{~s}$, each time followed by $30 \mathrm{~s}$ on ice. Then, they were centrifuged for 5 min at $4{ }^{\circ} \mathrm{C}$ and $1000 \times \mathrm{g}$, supernatants were transferred to fresh tubes and the lysates were assayed for protein concentration using Bradford solution as described in section 2.16.1.

$200 \mu \mathrm{g}$ of yeast cell homogenate were used per assay in a final volume of $100 \mu \mathrm{l}$ DGAT assay buffer also containing $6 \mathrm{nmol}\left[1-{ }^{14} \mathrm{C}\right]$-acyl-CoA (80 $\left.000 \mathrm{dpm}\right)$ and $10 \mu \mathrm{l}$ DAG/MAG-CHAPS solution (0.5 mM diacylglycerol or monoacylglycerol and $10 \mathrm{mM} 3-$ [(3-cholamidopropyl)dimethylammonio]-1-propanesulfonate (CHAPS) in DGAT assay buffer; The DAG/MAG-CHAPS solution was prepared beforehand by drying the diacylglycerol or monoacylglycerol moiety under streaming nitrogen, then adding CHAPS and DGAT assay buffer und treating this mixture for $15 \mathrm{~min}$ with ultrasound. The solution could be stored at $-20{ }^{\circ} \mathrm{C}$, prior to usage it was shaken strongly and again treated for $10 \mathrm{~min}$ with ultrasound). Samples were incubated at $30{ }^{\circ} \mathrm{C}$. At time points indicated for each experiment, reactions were stopped by addition of $100 \mu \mathrm{l} 0.9 \%(\mathrm{w} / \mathrm{v}) \mathrm{NaCl}$ solution and $300 \mu \mathrm{l}$ chloroform. Lipids were extracted by shaking for $3 \mathrm{~min}$ and samples were centrifuged for $5 \mathrm{~min}$ at $11000 \times \mathrm{g}$ to separate phases. The organic phase was transferred to a fresh tube and samples were reextracted with $300 \mu$ chloroform. Organic phases were combined, dried under streaming nitrogen and resuspended in $20 \mu \mathrm{l}$ chloroform/methanol $(1: 1, \mathrm{v} / \mathrm{v})$.

\subsubsection{LPLAT activity assays}

LPLAT activity assays were performed on the basis of (Stahl et al., 2008; Stalberg et al., 2009). Cell pellets of $50 \mathrm{ml}$ expression cultures were washed with $0.15 \mathrm{M} \mathrm{NaCl}$ solution, then resuspended in $1 \mathrm{ml}$ ice cold LPLAT sample buffer $(20 \mathrm{mM}$ Tris- $\mathrm{HCl}$, 
$\mathrm{pH} 7.6 ; 1 \mathrm{mM}$ EDTA) and transferred to $2 \mathrm{ml}$-tubes. Samples were centrifuged for $1 \mathrm{~min}$ at $4{ }^{\circ} \mathrm{C}$ and maximum speed in a microcentrifuge. Supernatants were discarded and pellets were resuspended in $400 \mu \mathrm{L}$ LPLAT sample buffer. An equal amount of glass beads was added and samples were vortexed eight times for $30 \mathrm{~s}$, each time followed by $30 \mathrm{~s}$ on ice. Then they were centrifuged for $5 \mathrm{~min}$ at $4{ }^{\circ} \mathrm{C}$ and $1000 \times \mathrm{g}$, supernatants were transferred to fresh tubes and lysates were assayed for protein concentration using Bradford solution as described in section 2.16.1.

For testing the forward reaction, $100 \mu \mathrm{g}$ of yeast cell homogenate were used per assay in a final volume of $100 \mu \mathrm{L}$ LPLAT assay buffer $(25 \mathrm{mM}$ Tris- $\mathrm{HCl}, \mathrm{pH} 7.6 ; 0.2 \mathrm{M} \mathrm{KCl})$ also containing $0.2 \mathrm{mM}$ lyso-phospholipid $(0.2 \mathrm{M}$ stock solutions were dissolved in ethanol/ $/ \mathrm{ddH}_{2} \mathrm{O}(1: 1, \mathrm{v} / \mathrm{v})$ and heated to $60{ }^{\circ} \mathrm{C}$ prior to usage) and $0.1 \mathrm{mM}\left[1-{ }^{14} \mathrm{C}\right]$-acylCoA (80 $000 \mathrm{dpm})$. In some reactions, total lipid extracts from $100 \mathrm{ml}$-cultures of $O$. tauri or O. lucimarinus were utilized as putative acyl acceptor instead of lysophospholipids. Samples were incubated at $23^{\circ} \mathrm{C}$ or $30^{\circ} \mathrm{C}$ for $5-60 \mathrm{~min}$, reactions were stopped by addition of $400 \mu \mathrm{l}$ methanol/chloroform/acetic acid (50:50:1, v/v/v) and $40 \mu \mathrm{l}$ $\mathrm{dd}_{2} \mathrm{O}$. Lipids were extracted by shaking for $3 \mathrm{~min}$ and samples were centrifuged for $5 \mathrm{~min}$ at $11000 \times \mathrm{g}$ to separate phases. The organic phase was transferred to fresh tubes and samples were reextracted with $200 \mu$ l chloroform. Organic phases were combined, dried under streaming nitrogen and resuspended in $20 \mu \mathrm{l}$ chloroform.

For assaying the LPLAT reverse reaction, $0.1 \mathrm{mM}$ di-18:2(n-6)-PC containing 80000 dpm di-[1- $\left.{ }^{14} \mathrm{C}\right]-18: 1(\mathrm{n}-9)-\mathrm{PC}$ and $80000 \mathrm{dpm}$ sn-1-16:0-sn-2-[1- $\left.{ }^{14} \mathrm{C}\right]-18: 2(\mathrm{n}-6)-\mathrm{PC}$ were mixed in $100 \mu \mathrm{L}$ LPLAT assay buffer with $2 \mathrm{mM}$ CoA, $0.2 \mathrm{mM}$ 18:1(n-9)-CoA, $10 \mathrm{mg} / \mathrm{ml}$ BSA and $50 \mu \mathrm{g}$ cell homogenate. Samples were incubated at $30^{\circ} \mathrm{C}$ for 1 hour and reactions were stopped with $100 \mu$ lethanol/acetic acid $(1: 1, \mathrm{v} / \mathrm{v})$.

For testing thioesterase activity, $100 \mu \mathrm{g}$ of yeast cell homogenate were used per assay in a final volume of $100 \mu \mathrm{L}$ LPLAT assay buffer containing $0.1 \mathrm{mM}\left[1-{ }^{14} \mathrm{C}\right]$-acyl-CoA (80 $000 \mathrm{dpm}$ ) and $10 \mathrm{mg} / \mathrm{ml} \mathrm{BSA}$. Samples were incubated at $30{ }^{\circ} \mathrm{C}$ for $20 \mathrm{~min}$, reactions were stopped by addition of $400 \mu \mathrm{l}$ methanol/chloroform/acetic acid (50:50:1,

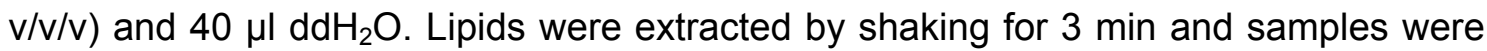
centrifuged for $5 \mathrm{~min}$ at $11000 \times \mathrm{g}$ to separate phases. The organic phase was transferred to fresh tubes and samples were reextracted with $200 \mu \mathrm{l}$ chloroform. Organic phases were combined, dried under streaming nitrogen and resuspended in $20 \mu \mathrm{l}$ chloroform. 


\subsection{Lipid analysis}

\subsubsection{Lipid extraction methods}

\subsubsection{Lipid extraction from algae pellets}

$200 \mathrm{ml}$-algae cultures were harvested by centrifugation at $3220 \times \mathrm{g}$ for $15 \mathrm{~min}$. Supernatants were discarded and $10 \mu \mathrm{g}$ tri-pentadecanoin were added to the algae pellets as internal standard. Lipids were extracted from pellets by vigorous vortexing in $5 \mathrm{ml}$ chloroform/methanol $(1: 2, \mathrm{v} / \mathrm{v})$ containing $3 \%(\mathrm{v} / \mathrm{v})$ acetic acid with $2 \mathrm{ml}$ glass beads and incubating the samples overnight at $4{ }^{\circ} \mathrm{C}$ (method according to (Folch et al., 1957)). The resulting lipid extracts were filtered through cotton wool. The algae pellets were reextracted with $5 \mathrm{ml}$ chloroform/methanol $(2: 1, \mathrm{v} / \mathrm{v})$ containing $3 \%(\mathrm{v} / \mathrm{v})$ acetic acid and again incubated overnight at $4{ }^{\circ} \mathrm{C}$. The resulting second lipid extracts were also filtered through cotton wool and combined with the first lipid extracts. Remaining lipids within the algae pellets were extracted by incubation for $20 \mathrm{~min}$ at $23{ }^{\circ} \mathrm{C}$ in $2 \mathrm{ml}$ hexane. Again, the resulting supernatants were filtered through cotton wool and combined with first and second lipid extracts. After drying samples under streaming nitrogen, samples were subjected to fatty acid methyl ester (FAME) derivatization.

\subsubsection{Lipid extraction from yeast pellets}

For fast lipid extraction from yeast cultures, $10 \mu \mathrm{g}$ tri-heptadecanoin, di-heptadecanoylPC, di-heptadecanoyl-PE and di-heptadecanoyl-PS, respectively, were added to cell pellets from $100 \mathrm{ml}$-expression cultures. Lipids were extracted from pellets by consecutive vigorous vortexing in $3 \mathrm{ml}$ methanol and $6 \mathrm{ml}$ chloroform/methanol $(1: 1, \mathrm{v} / \mathrm{v})$ with $2 \mathrm{ml}$ glass beads for $20 \mathrm{~min}$. Samples were centrifuged at $1500 \times g$ for $5 \mathrm{~min}$, lipid extracts were filtered through cotton wool. Pellets were vortexed with $3 \mathrm{ml}$ hexane/diethyl ether/formic acid (65:35:1, v/v/v) and samples were incubated for $20 \mathrm{~min}$ at $23{ }^{\circ} \mathrm{C}$. Resulting lipid extracts were combined with the previous ones. After drying samples under streaming nitrogen, remaining lipids were dissolved in $50 \mu$ l chloroform.

In an alternative procedure that was used for lipid extraction from yeast, cell pellets of $20 \mathrm{ml}$-expression cultures were extracted by vigorous vortexing in $5 \mathrm{ml}$ chloroform/methanol $(1: 2, \mathrm{v} / \mathrm{v})$ with $2 \mathrm{ml}$ glass beads for 4 hours at $4{ }^{\circ} \mathrm{C}$. Samples were centrifuged for $5 \mathrm{~min}$ at $1500 \times \mathrm{g}$ and $4{ }^{\circ} \mathrm{C}$. Supernatants were transferred to fresh tubes. Cell pellets were reextracted with chloroform/methanol $(2: 1, \mathrm{v} / \mathrm{v})$ overnight at $4{ }^{\circ} \mathrm{C}$ while shaking. Samples were centrifuged again as described before and supernatants were combined with the first lipid extracts. After drying samples under streaming nitrogen, remaining lipids were dissolved in $50 \mu$ chloroform.

Another method used was the one developed by (Ejsing et al., 2009). Cell pellets from $10 \mathrm{ml}$-expression cultures were resuspended in $1 \mathrm{ml} 150 \mathrm{mM} \mathrm{NH} \mathrm{NCO}_{3} .10 \mu \mathrm{g}$ tri- 
decapentanoin and di-heptadecanoyl-PC were added and samples were vigorously vortexed. $5 \mathrm{ml}$ chloroform/methanol $(17: 1, \mathrm{v} / \mathrm{v})$ were added and samples were shaken for 2 hours at $4{ }^{\circ} \mathrm{C}$. Phases were separated by centrifugation at $4{ }^{\circ} \mathrm{C}$ and $1500 \times \mathrm{g}$ for $5 \mathrm{~min}$. Organic phases were transferred to fresh glass tubes and samples were reextracted with $5 \mathrm{ml}$ chloroform/methanol $(17: 1, \mathrm{v} / \mathrm{v})$ for another hour. Organic phases were combined and dried under streaming nitrogen.

\subsubsection{A. thaliana seeds}

$20 \mathrm{mg} \mathrm{A}$. thaliana seeds were homogenized in methanol/chloroform/acetic acid (50:50:1, v/v/v). $20 \mu \mathrm{g}$ tri-decapentanoin and di-heptadecanoyl-PC, respectively, were added as internal standards. Samples were incubated for 1 hour, then they were centrifuged for $5 \mathrm{~min}$ at $1500 \times \mathrm{g}$ and supernatants were transferred to fresh tubes. Pellets were reextracted with $1 \mathrm{ml}$ hexane and incubated for $20 \mathrm{~min}$ at $23{ }^{\circ} \mathrm{C}$. Samples were centrifuged as described before, both lipid extracts were combined and evaporated under streaming nitrogen. Remaining lipids were resuspended in $50 \mu \mathrm{l}$ chloroform.

\subsubsection{Thin layer chromatography (TLC)}

TLC was performed in $20 \times 20 \times 10 \mathrm{~cm}$ vertical glass chambers with $100 \mathrm{ml}$ developing solvent and silica gel thin layer plates with the fineness 60 (Kieselgel 60, $20 \times 20 \mathrm{~cm}$; Merck, Darmstadt, Germany).

\subsubsection{TLC of radioactive lipid extracts}

$5 \mu \mathrm{l}$ of total lipid extracts from DGAT and MGAT activity assays were applied onto a thin layer plate and separated by a two step TLC using standard lipids for identification of different lipid classes by co-migration. First, phospholipids were separated using acetic acid methyl ester/isopropanol/chloroform/methanol/0.25\% (w/v) $\mathrm{KCl}$ $(25: 25: 28: 10: 7, \mathrm{v} / \mathrm{v} / \mathrm{v} / \mathrm{v} / \mathrm{v})$. After the solvent had reached the center, the plate was taken out of the tank and dried. Then, the plate was put into a second chamber filled with hexane/diethyl ether/ acetic acid (80:20:1, v/v/v) as developing solvent in order to separate neutral lipids.

$5 \mu \mathrm{l}$ of total lipid extracts from LPLAT activity assays were applied onto a thin layer plate and separated by TLC using standard lipids for identification by co-migration. As developing solvent, chloroform/methanol/acetic acid/dd $\mathrm{H}_{2} \mathrm{O}(85: 15: 10: 3.5$, v/v/v/v) was used.

For testing the reverse reaction in LPLAT activity assays, $20 \mu \mathrm{l}$ per sample were directly put on a thin layer plate, dried and developed in butanol/acetic acid/ $/ \mathrm{ddH}_{2} \mathrm{O}$ $(5: 2: 3, \mathrm{v} / \mathrm{v} / \mathrm{v})$. Radiolabelled lipids were visualized using a phosphor storage screen and a phosphoimager (Fuji FLA-3000, Raytest, Straubenhardt, Germany). 


\subsubsection{TLC of non-radioactive lipid extracts}

Lipid extracts were applied onto thin layer plates and separated by different solvents. Neutral lipids were separated using hexane/diethyl ether/acetic acid (80:20:1, v/v/v), phospholipids were separated using chloroform/methanol/acetic acid (65:25:8, v/v/v). Phospholipids were separated from monogalactosyldiacylglycerol using acetic acid methyl ester/isopropanol/chloroform/methanol/0.25\% (w/v) $\mathrm{KCl} \quad(25: 25: 25: 10: 9$, $\mathrm{v} / \mathrm{v} / \mathrm{v} / \mathrm{v} / \mathrm{v})$. Standard lipids were applied to identify the different lipid classes by comigration.

For analytical purposes, thin layer plates were incubated in $\mathrm{CuSO}_{4}$ solution $(10 \mathrm{~g}$ $\mathrm{CuSO}_{4} \times 5 \mathrm{H}_{2} \mathrm{O}, 92 \mathrm{ml} \mathrm{H}_{2} \mathrm{O}, 8 \mathrm{ml} \mathrm{H}_{3} \mathrm{PO}_{4}$ ) using the Chromatogram Immersion Device III (Camag, Muttenz, Switzerland). Afterwards, plates were heated on a TLC Heating Plate (Camag, Muttenz, Switzerland) to $170{ }^{\circ} \mathrm{C}$ for visualization of lipids.

For preparative purposes, thin layer plates were sprayed with $0.2 \%(w / v)$ 8-anilino-1naphthalene-sulphonic acid (ANS) using the TLC Spray Cabinet/Sprayer (Camag, Muttenz, Switzerland). Under UV light $(\lambda=254$ and $365 \mathrm{~nm}$ ) lipids were visualized and could be marked in order to scrape the desired fractions from the plate for derivatization of fatty acids.

\subsubsection{Isolation and derivatization of fatty acids}

\subsubsection{Derivatization of fatty acids from yeast cell sediments}

Total fatty acids (unbound and esterified to lipids) from cell pellets of 5-10 ml yeast expression cultures were derivatized into their respective FAMEs via acidic hydrolysis (Miquel and Browse, 1992). 2 ml FAME solution (2.75 \% (v/v) $\mathrm{H}_{2} \mathrm{SO}_{4}(95-97 \%$ ) and $2 \%(\mathrm{v} / \mathrm{v})$ dimethoxy propane in methanol) were added to the washed yeast cell pellets. Samples were vortexed and incubated for 1 hour at $80^{\circ} \mathrm{C}$. Afterwards, $200 \mu \mathrm{l} \mathrm{M} \mathrm{NaCl}$ solution and $2 \mathrm{ml}$ hexane were added. Samples were vortexed and then centrifuged at $1500 \times g$ for $5 \mathrm{~min}$. The upper phase was transferred to new glass tubes and dried under streaming nitrogen. The remaining FAMEs were then dissolved in 10-50 $\mu \mathrm{l}$ acetonitrile and transferred to gas chromatography (GC) vials for subsequent analysis.

\subsubsection{Derivatization of fatty acids from pooled seeds}

Total fatty acids (unbound and esterified to lipids) from $3 \mathrm{mg} A$. thaliana seeds were also derivatized into their respective FAMEs based on the procedure of acidic hydrolysis (Miquel and Browse, 1992). 20-40 $\mu \mathrm{g}$ tri-decapentanoin and $2 \mathrm{ml}$ FAME-toluol solution $\left(2.75 \%(\mathrm{v} / \mathrm{v}) \mathrm{H}_{2} \mathrm{SO}_{4}(95-97 \%)\right.$ and $2 \%(\mathrm{v} / \mathrm{v})$ dimethoxy propane in methanol/toluol $(2: 1, v / v))$ were added to the seeds, samples were incubated and extracted as described above (2.18.3.1). After evaporation of the solvent, methyl esters were dissolved in $200 \mu \mathrm{l}$ acetonitrile for GC measurement. 
2.18.3.3 Derivatization of fatty acids from lipid extracts or isolated lipid fractions

Fatty acids esterified to lipids were derivatized into their respective FAMEs by transesterification (Hornung et al., 2002). $333 \mu \mathrm{l}$ toluol/methanol (1:2, v/v) and $167 \mu \mathrm{l} 0.5 \mathrm{M}$ $\mathrm{NaOCH}_{3}$ were added to the samples, which were incubated for $20 \mathrm{~min}$ at $23^{\circ} \mathrm{C}$. Then, $100 \mu \mathrm{l} 5 \mathrm{M} \mathrm{NaCl}$ solution were added and methyl esters were extracted with $2 \mathrm{ml}$ hexane. Samples were centrifuged at $1500 \times g$ for $5 \mathrm{~min}$. The upper phase was transferred to fresh glass tubes and dried under streaming nitrogen. Remaining methyl esters were then dissolved in 10-50 $\mu$ lacetonitrile for GC analysis.

\subsubsection{Derivatization of fatty acids from single $A$. thaliana or $C$. sativa seeds}

Single seeds were treated with trimethylsulfoniumhydroxide (TMSH) according to (Müller et al., 1990) in order to obtain FAMEs from unbound fatty acids as well as from fatty acids esterified to lipids. Single seeds were ground in 5-20 $\mu \mathrm{TMSH}$ in a GC vial. In case of $C$. sativa seeds, $10 \mu \mathrm{l}$ of the resulting supernatant was transferred to a fresh vial to avoid plugging of the GC injection needle. Transesterification occurred during incubation at $23^{\circ} \mathrm{C}$. At the same time, the TMSH evaporated and the methyl esters could be dissolved in 10-20 $\mu$ l acetonitrile for GC measurement.

\subsubsection{Gas chromatography (GC)}

FAMEs were analysed via a gas chromatograph coupled to a flame ionisation detector (FID) (6890 series GC system; Agilent, Waldbronn, Germany) using a capillary DB-23 column (30 m x $0.25 \mathrm{~mm}, 0.25 \mu \mathrm{m}$ coating thickness, J\&W Scientific, Agilent, Waldbronn, Germany) according to (Hornung et al., 2002). Helium was used as carrier gas with a flow of $1 \mathrm{ml} / \mathrm{min}$. The temperature gradient was $150{ }^{\circ} \mathrm{C}$ for $1 \mathrm{~min}, 150-200{ }^{\circ} \mathrm{C}$ at $4 \mathrm{~K} / \mathrm{min}, 200-250{ }^{\circ} \mathrm{C}$ at $5 \mathrm{~K} / \mathrm{min}$ and $250{ }^{\circ} \mathrm{C}$ for $6 \mathrm{~min}$. As retention time standards, FAME-Mix (C4-C24; Sigma, München) as well as Menhaden oil (Sigma, München) derivatized into FAMEs were injected before samples were run. Injection volumes depended on the concentration of FAMEs in the sample. Data were processed using ChemStation Rev. A09.03 (Agilent, Waldbronn, Germany).

FID signals, which could not be identified by GC were further analysed by their mass spectra using a 6890 gas chromatograph/5973 mass selective detector system (Agilent, Waldbronn). GC conditions were the same as for GC analysis. As MS conditions, an electron energy of $70 \mathrm{eV}$, an ion source temperature of $230{ }^{\circ} \mathrm{C}$ and a temperature of $260{ }^{\circ} \mathrm{C}$ for the transfer line were used. Resulting spectra were compared with the Lipid Library of the Scottish Crop Science research Institute (http://www.lipidlibrary.co.uk/index.html) to identify unknown substances.

\subsubsection{Extraction, derivatization and analysis of acyl-CoAs}

For analysis of acyl-CoAs from yeast pellets, the method developed by (Larson and Graham, 2001) was used. During this procedure, isolated acyl-CoA esters are converted into etheno-derivates which are then separated by reversed-phase high per- 
formance liquid chromatography (HPLC) and can be detected by a fluorescence detector. Extraction of acyl-CoAs was performed on the basis of (Rosendal and Knudsen, 1992). To cell pellets from $10 \mathrm{ml}$-yeast cultures, $0.25 \mathrm{nmol}$ heptadecanoyl-CoA as internal standard, $800 \mu \mathrm{ldd} \mathrm{H}_{2} \mathrm{O}, 3 \mathrm{ml}$ chloroform/methanol $(2: 1, \mathrm{v} / \mathrm{v})$ and $2 \mathrm{ml}$ glass beads were added. Samples were vigorously vortexed at $4{ }^{\circ} \mathrm{C}$ for $20 \mathrm{~min}$. $1 \mathrm{ml}$ chloroform and $1 \mathrm{ml} \mathrm{ddH_{2 }} \mathrm{O}$ were added and samples were vortexed for $30 \mathrm{~s}$. Then they were centrifuged for $10 \mathrm{~min}$ at $3320 \times \mathrm{g}$ and $4{ }^{\circ} \mathrm{C}$. Upper and lower phase were discarded, the interphase was dried under streaming nitrogen. $400 \mu \mathrm{l}$ extraction buffer $(2 \mathrm{ml}$ isopropanol, $2 \mathrm{ml} 50 \mathrm{mM} \mathrm{KH}_{2} \mathrm{PO}_{4}, 50 \mu \mathrm{l}$ acetic acid, $80 \mu \mathrm{l} 50 \mathrm{mg} / \mathrm{ml}$ BSA (fatty acid free, Sigma, Munich)), $10 \mu \mathrm{l}$ saturated $\left(\mathrm{NH}_{4}\right)_{2} \mathrm{SO}_{4}$ and $1200 \mu \mathrm{l}$ methanol/chloroform $(2: 1, \mathrm{v} / \mathrm{v})$ were added to the samples. These were vortexed on a shaker at $4{ }^{\circ} \mathrm{C}$ for $20 \mathrm{~min}$. Samples were then incubated for $20 \mathrm{~min}$ at $23{ }^{\circ} \mathrm{C}$ and afterwards centrifuged at $3320 \times \mathrm{g}$ for $5 \mathrm{~min}$. Supernatants were transferred to $1.5 \mathrm{ml}$-tubes and dried under streaming nitrogen. Samples were either frozen at $-80{ }^{\circ} \mathrm{C}$ or directly derivatized by addition of $300 \mu \mathrm{l}$ derivatization solution $(50 \%(\mathrm{v} / \mathrm{v})$ chloroacetaldehyde solution $(7.9 \mathrm{M})$ and $0.5 \%(\mathrm{w} / \mathrm{v}) \mathrm{SDS}$ in $\mathrm{ddH}_{2} \mathrm{O}$, adjusted to $\mathrm{pH} 4$ with $0.15 \mathrm{M}$ citrate buffer) and incubation for $20 \mathrm{~min}$ at $85^{\circ} \mathrm{C}$. Samples could either be measured directly or stored at $-20^{\circ} \mathrm{C}$ until analysis was performed.

Etheno-derivates were separated at 300 bar by using the 1100 Series HPLC system (Agilent, Waldbronn, Germany), a LUNA column $(150 \times 2 \mathrm{~mm}$, coated with $5 \mu \mathrm{m}$ phenyl-hexyl silicium particles; Phenomenex, Aschaffenburg, Germany) as main column, a phenyl propyl guard $(4 \times 2 \mathrm{~mm}$ ) as precolumn (Phenomenex, Aschaffenburg, Germany) and a fluorescence detector $(\lambda($ excitation $)=230 \mathrm{~nm}, \lambda($ emission $)=420 \mathrm{~nm})$. 10-20 $\mu \mathrm{l}$ of each sample were injected for every measurement. Standard acyl-CoAs were also derivatized and used for identification of peaks in the samples. The gradient for separation and elution of the different etheno-derivates is given below.

$\begin{array}{cccccc}\text { Time (min) } & \mathbf{A}(\%) & \mathbf{B}(\%) & \mathbf{C ~ ( \% )} & \mathbf{D}(\%) & \text { Flow rate (ml/min) } \\ 0 & 90 & 10 & 0 & 0 & 0.4 \\ 5 & 20 & 80 & 0 & 0 & 0.4 \\ 5.1 & 20 & 0 & 80 & 0 & 0.4 \\ 7 & 0 & 0 & 97 & 3 & 0.2 \\ 10 & 0 & 0 & 95 & 5 & 0.2 \\ 10.1 & 0 & 0 & 95 & 5 & 0.2 \\ 50 & 0 & 0 & 55 & 45 & 0.2 \\ 51.1 & 0 & 0 & 0 & 100 & 0.4 \\ 52 & 0 & 0 & 0 & 100 & 0.4 \\ 62 & 0 & 0 & 0 & 100 & 0.4 \\ 62.1 & 90 & 10 & 0 & 0 & 0.4 \\ 65 & 90 & 10 & 0 & 0 & 0.4\end{array}$


eluent $A$ : $\quad \mathrm{H}_{2} \mathrm{O} /$ acetic acid $(100: 1, \mathrm{v} / \mathrm{v})$

eluent B: $\quad$ acetonitrile/ $\mathrm{H}_{2} \mathrm{O} /$ acetic acid $(90: 9: 1, \mathrm{v} / \mathrm{v} / \mathrm{v})$

eluent C: $\quad \mathrm{H}_{2} \mathrm{O} /$ triethylamine $(100: 0.25, \mathrm{v} / \mathrm{v})$

eluent D: $\quad$ acetonitrile/ $\mathrm{H}_{2} \mathrm{O}(90: 10, \mathrm{v} / \mathrm{v})$ 


\section{Results}

Marine microalgae are primary producers of VLCPUFAs and therefore potential gene donors for genes encoding enzymes active in VLCPUFA biosynthesis or distribution. Microalgal acyltransferases for example might be useful to improve the substrate feed for the biosynthetic enzymatic activities or to mediate transfer of the end products into a desired lipid fraction. Desaturases or elongases, on the other hand, are directly required for the synthesis of VLCPUFAs. In previous investigations, the closely related microalgal species $O$. tauri, $O$. Iucimarinus and $M$. squamata turned out to be useful gene donors for both acyltransferases and desaturases (Domergue et al., 2005; Hoffmann et al., 2008; Wagner, 2008).

\subsection{Characterization of microalgal acyltransferases in yeast}

The prasinophyte O. tauri was shown to accumulate high amounts of DHA in its TAG fraction. Because of this finding, the organism was believed to be a valuable model to analyze mechanisms of VLCPUFA distribution into its neutral lipids (Wagner et al., 2010). Therefore, nucleotide sequences encoding different acyltransferases putatively involved in this mechanism were isolated by Dr. Martin Wagner. Due to its close relationship to O. tauri, also O. lucimarinus was used as gene donor for a potential acyltransferase (Wagner, 2008). The identified sequences were already partly analyzed during the beforehand mentioned work. However, experiments were still missing for the complete characterization and were thus conducted in the presented work.

\subsubsection{Characterization of the putative acyl-CoA:Iysophosphatidylcholine acyltransferases OILPCATp and OtLPCATp}

OtLPCAT and OILPCAT were identified by searching through the genome of O. tauri and O. lucimarinus with ALE1, a yeast LPCAT (Benghezal et al., 2007; Chen et al., 2007; Jain et al., 2007; Riekhof et al., 2007; Tamaki et al., 2007). Over-expression of the completely codon-optimized OILPCAT in yeast led to solid protein amounts detectable in Western Blot and was able to partially complement the yeast mutant strain BY4741 ale1 $\triangle$ deficient in intrinsic LPCAT activity (Wagner, 2008). OtLPCAT was isolated and partly codon-optimized by Dr. Mareike Heilmann, but was not characterized so far. Therefore, it was checked first whether over-expression of OtLPCAT led to detectable protein levels in yeast. For this purpose, both sequences directly fused to a V5-epitope sequence at their 3'-end in pYES2/CT were expressed under the control of GAL1 promoter in the yeast strain BY4741 ale1A. As positive control, the $ß-$ galactosidase sequence $L a c Z$ fused to a V5-epitope sequence in pYES2/CT and as negative control, empty pYES2/CT vector were transformed into the same strain. Ex- 
pression cultures were grown for two days at $23{ }^{\circ} \mathrm{C}$. Afterwards, cultures were harvested and protein lysates were analyzed by Western Blot for V5-epitope. As can be seen in Figure $4 \mathrm{~A}$, the positive control showed a band at $120 \mathrm{kDa}$, which represents the expected size of the ß-galactosidase protein with carboxyl-terminal V5-epitope. In contrast, protein lysates from both OtLPCAT- and OILPCAT-expressing cultures did not exhibit bands at the expected protein sizes of $58 \mathrm{kDa}$ and $50 \mathrm{kDa}$, respectively. The observed band pattern was not different from the one deriving from the negative control culture lysates and thus seemed to result from unspecific binding of the antibody. OILPCAT and OtLPCAT sequences were also cloned into pUG36 in order to obtain amino-terminal GFP-tagged proteins. Western Blot experiments with cell lysates expressing these constructs also led to negative results (data not shown). In addition, yeast cells expressing GFP-OILPCAT or GFP-OtLPCAT were observed under the microscope, but neither of the fusion proteins could be detected (data not shown).

A

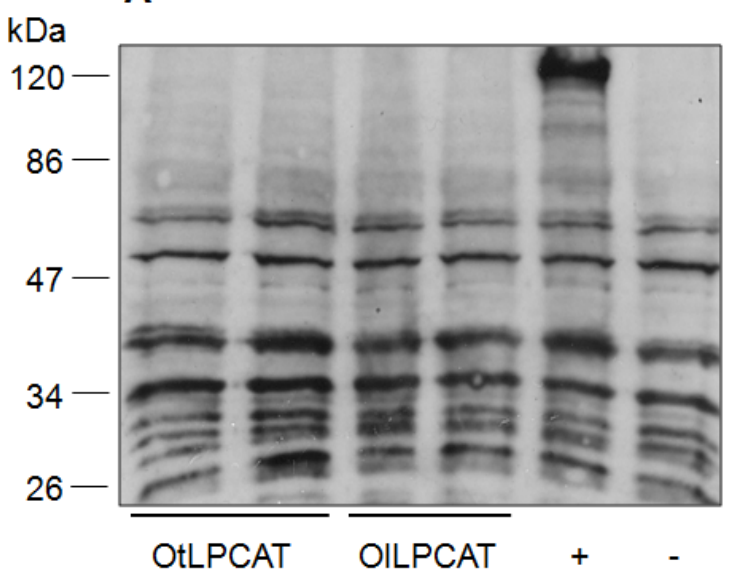

B

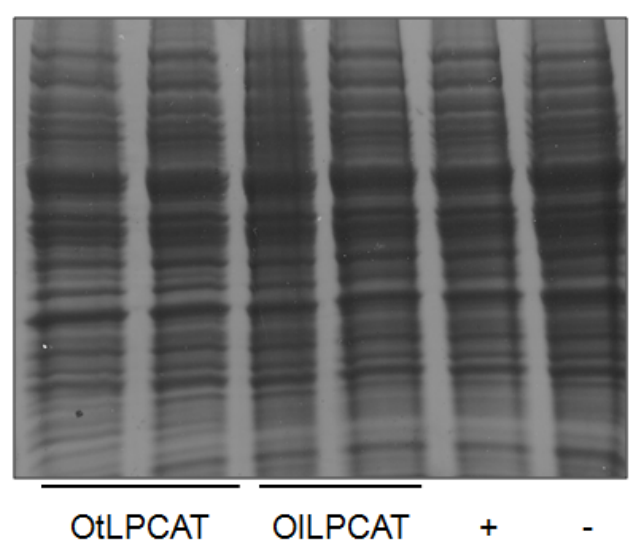

Figure 4. Protein amounts after over-expression of OtLPCAT-V5 and OILPCAT-V5 OtLPCAT-V5-pYES2/CT, OILPCAT-V5-pYES2/CT, LacZ-V5-pYES2/CT (+; positive control) or pYES2/CT (-; negative control) were expressed for two days at $23{ }^{\circ} \mathrm{C}$ in BY4741 ale1 1 . Cells were lysed and total lysates were analyzed. (A) Western Blot and (B) corresponding Coomassie gel as loading control. OtLPCAT-V $5 p$ was expected to be $58 \mathrm{kDa}$, OILPCAT-V5p was expected to be $50 \mathrm{kDa}$ and LacZ-V5p $120 \mathrm{kDa}$ in size. The experiment was performed once with two independent clones. 
Nevertheless, in previous experiments OILPCAT expression was found to partially complement the yeast mutant strain BY4741 ale1D (Wagner, 2008). Also, expression of sequences lacking the epitope sequence might result in higher protein levels in yeast compared to the V5-epitope fusions of OtLPCATp and OILPCATp. Therefore, in vitro LPLAT assays were performed with cell homogenates from cultures expressing the microalgal sequences without epitope to elucidate the catalyzed reaction and substrate specificities of the potential acyltransferases. The completely codon-optimized sequence of OILPCAT and the partly codon-optimized sequence of OtLPCAT were expressed under the control of GAL1 promoter in pYES2/CT in the yeast strain BY4741 ale1 $\triangle$. As negative control, pYES2/CT was expressed in the mutant strain. As positive controls, the construct ALE1-pYES2.1/TOPO (by courtesy of Dr. U. Ståhl) in the mutant strain and pYES2/CT in BY4741 wild type cells were expressed. Expression cultures were grown either for one day at $30{ }^{\circ} \mathrm{C}$, for two days at $23{ }^{\circ} \mathrm{C}$ or for three days at $16{ }^{\circ} \mathrm{C}$. Afterwards, yeast cells were harvested and used for homogenate preparation. Resulting homogenates were tested with different lyso-phospholipids as acyl acceptors and various radiolabeled acyl-CoAs as acyl donors in the LPLAT activity assay. The result of an exemplary experiment is depicted in Figure 5. Whereas both positive controls exhibited considerable amounts of radiolabeled and thus newly formed PC, expression of neither OtLPCAT nor OILPCAT could complement the PC-deficient phenotype of BY4741 ale1 $\Delta$ in vitro. Varying expression and assay temperatures as well as different acyl donor and acceptor combinations did not result in detectable activity of the putative microalgal LPCATs. In Table 1, an overview about different combinations of acyl acceptors and acyl donors is given. Also, lipid extracts from 0 . tauri or 0 . lucimarinus were tested in some experiments as possible acyl acceptors instead of defined lyso-phospholipids, but no differences could be observed between lysates from cultures expressing OtLPCAT or OILPCAT in comparison to those from cultures expressing empty vector (data not shown).

Also, the reverse LPLAT reaction of cell homogenates was assayed by using radiolabeled PC and CoA under excess of 18:1(n-9)-CoA. Furthermore, thioesterase activity was examined by incubation of different radiolabeled acyl-CoAs together with cell homogenates. In both assays, no catalytic activity for OtLPCATp and OILPCATp could be shown (data not shown).

Summing up these results, both OILPCAT and OtLPCAT did not reveal detectable protein levels upon expression in yeast when fused to the V5-epitope sequence and for both putative acyltransferases neither LPLAT nor thioesterase activity could be shown in vitro when the native proteins without the V5-epitope were used. 


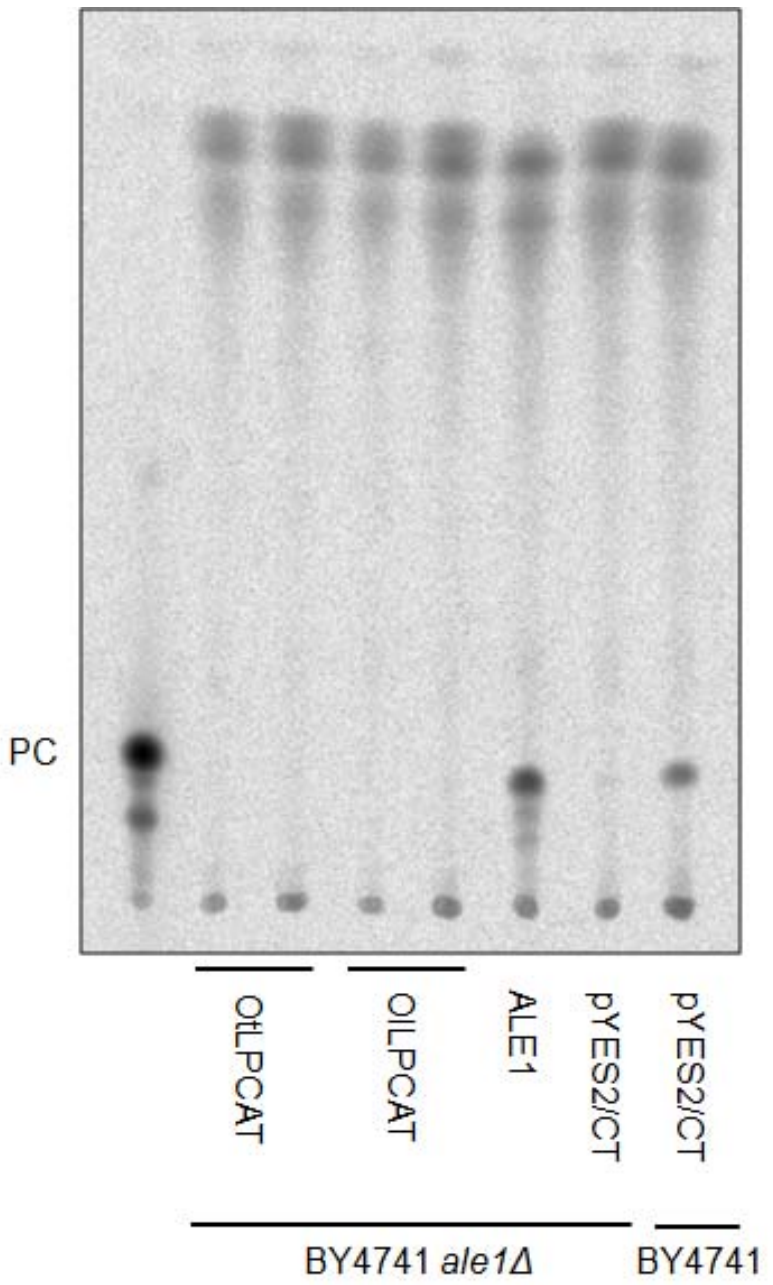

Figure 5. OtLPCATp and OILPCATp activity in vitro

pYES2/CT (negative control), OtLPCAT-pYES2/CT, OILPCAT-pYES2/CT or ALE1pYES2.1/TOPO (positive control) were expressed for one day at $30^{\circ} \mathrm{C}$ in the yeast mutant BY4741 ale1 $\Delta$ or in the wild type strain BY4741 as indicated. Cell homogenates were prepared and incubated with $\left[1-{ }^{14} \mathrm{C}\right]-18: 3(\mathrm{n}-3)-\mathrm{CoA}$ and lyso-16:0-PC. Reactions were incubated at $30{ }^{\circ} \mathrm{C}$ and stopped after five min. PC, phosphatidylcholine. The experiment was performed once with two independent clones for OtLPCATp and OILPCATp. 
Table 1. Overview about different in vitro assay conditions to test for OtLPCATp and OILPCATp activity

OtLPCAT-pYES2/CT, OILPCAT-pYES2/CT and positive as well as negative controls were expressed for one day at $30^{\circ} \mathrm{C}$ or for two days at $23^{\circ} \mathrm{C}$ or for three days at $16{ }^{\circ} \mathrm{C}$ in the yeast mutant BY4741 ale $1 \Delta$ or in the wild type strain BY4741. Cell homogenates were prepared and incubated with $\left[1-{ }^{14} \mathrm{C}\right]$-labeled CoAs and different lyso-phospholipids as indicated. Reactions were incubated at $30^{\circ} \mathrm{C}$ and stopped after five min or incubated at $23{ }^{\circ} \mathrm{C}$ for one hour. PC, phosphatidylcholine; PE, phosphatidylethanolamine; PG, phosphatidylglycerol; PA, phosphatidic acid; LE, total lipid extract. Numbers of independent experiments for each putative LPCAT are indicated (multiple clones or multiple expressions of the same clone).

\begin{tabular}{|lcccc|}
\hline & 18:1(n-9)-CoA & 18:3(n-3)-CoA & 18:3(n-6)-CoA & 20:4(n-6)-CoA \\
\hline 16:0-lyso-PC & 7 & 8 & & \\
\hline 16:0-lyso-PE & 2 & & & \\
\hline 16:0-lyso-PG & 2 & & & \\
\hline 18:0-lyso-PA & 2 & & 2 & 2 \\
\hline O. tauri LE & 2 & 2 & 2 & 2 \\
\hline O. lucimarinus LE & 2 & 2 & & \\
\hline
\end{tabular}

\subsubsection{In vitro studies of the acyl-CoA:diacylglycerol acyltransferase OtDGAT2Bp}

OtDGAT2B was identified by searching through the genome of O. tauri with DGAT2 nucleotide sequences from the fungus $M$. ramanniana (Lardizabal et al., 2001). Expression of the partly codon-optimized OtDGAT2B led to detectable protein levels in yeast and was found to complement the yeast mutant BY4741 dga1 $\Delta$ Iro1 $\Delta$ lacking the only DGAT and PDAT enzymes in yeast and thus being devoid of TAG. Furthermore, in vivo yeast expression experiments showed that the enzyme does not differentiate neither between (n-3)- and (n-6)-substrates nor between acyl-CoAs with 18 or 20 carbon atoms (Wagner et al., 2010). The remaining question was whether in vitro assays would confirm the previously in vivo obtained results concerning substrate specificity.

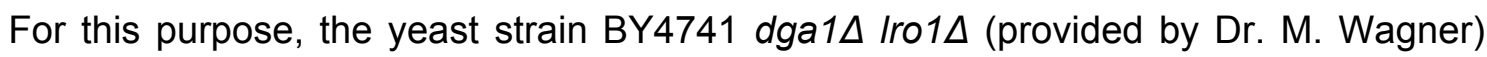
was used to additionally knockout the yeast gene for acyl-CoA thioesterase TES1 (Jones et al., 1999; Ntamack et al., 2009) by PCR-mediated gene deletion. This was done because TES1p might have interfered with the in vitro assay by cleavage of the externally provided acyl-CoA substrates. After successful gene deletion, pYES2/CTconstructs containing either the partly codon-optimized OtDGAT2B or, as positive control, the yeast DGAT cDNA sequence DGA1 (Oelkers et al., 2002; Sorger and Daum, 2002) under control of the GAL1 promoter (provided by Dr. M. Wagner) were trans-

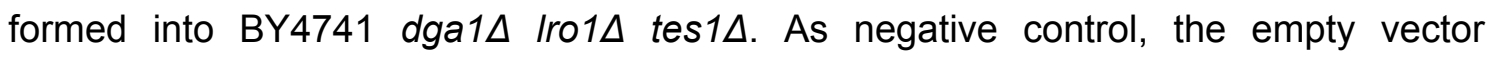
pYES2/CT was transformed into the same strain. Expression cultures were grown for one day at $30^{\circ} \mathrm{C}$. Afterwards, yeast cells were harvested and used for homogenate 
preparation. Resulting homogenates were tested with different DAGs as potential acyl acceptors and various radiolabeled acyl-CoAs as potential acyl donors in a DGAT activity assay. Results of two exemplary experiments are depicted in Figure 6. Figure $6 \mathrm{~A}$ shows TAG formation after utilization of di-18:1(n-9)-DAG as acyl acceptor together with $\left[1-{ }^{14} \mathrm{C}\right]-18: 1(\mathrm{n}-9)-\mathrm{CoA}$ as acyl donor, whereas Figure $6 \mathrm{~B}$ shows results after utilization of di-18:2(n-6)-DAG together with $\left[1-{ }^{14} \mathrm{C}\right]-18: 1(n-9)-C o A$. Both experiments led to a similar outcome. TAG formation was strongest after expression of endogenous $D G A 1$ in yeast. Even when the reaction was stopped immediately after addition of all components (0 min), TAG formation was observable for the positive control. In contrast, homogenates of OtDGAT2B-expressing cultures produced TAG in visible amounts no more than one hour after initiation of the experiment. In addition to $\left[1-{ }^{14} \mathrm{C}\right]-18: 1(n-9)$ CoA also $\left[1-{ }^{14} C\right]-18: 3(n-3)-C o A,\left[1-{ }^{14} C\right]-18: 3(n-6)-C o A$ as well as $\left[1-{ }^{14} C\right]-20: 4(n-6)-C o A$ were tested as putative acyl donors. Comparable TAG formation could be observed for homogenates from $D G A 1$-expressing cultures with all tested substrates. For homogenates from OtDGAT2B-expressing cultures, no differences in comparison to the negative control homogenates could be observed (data not shown).

In conclusion, OtDGAT2Bp in vitro preferred 18:1(n-9)-CoA to polyunsaturated fatty acyl-CoAs with 18 or 20 carbon atoms as acyl donor but did not differentiate between di-18:1(n-9)-DAG and di-18:2(n-6)-DAG as acyl acceptor. 
A

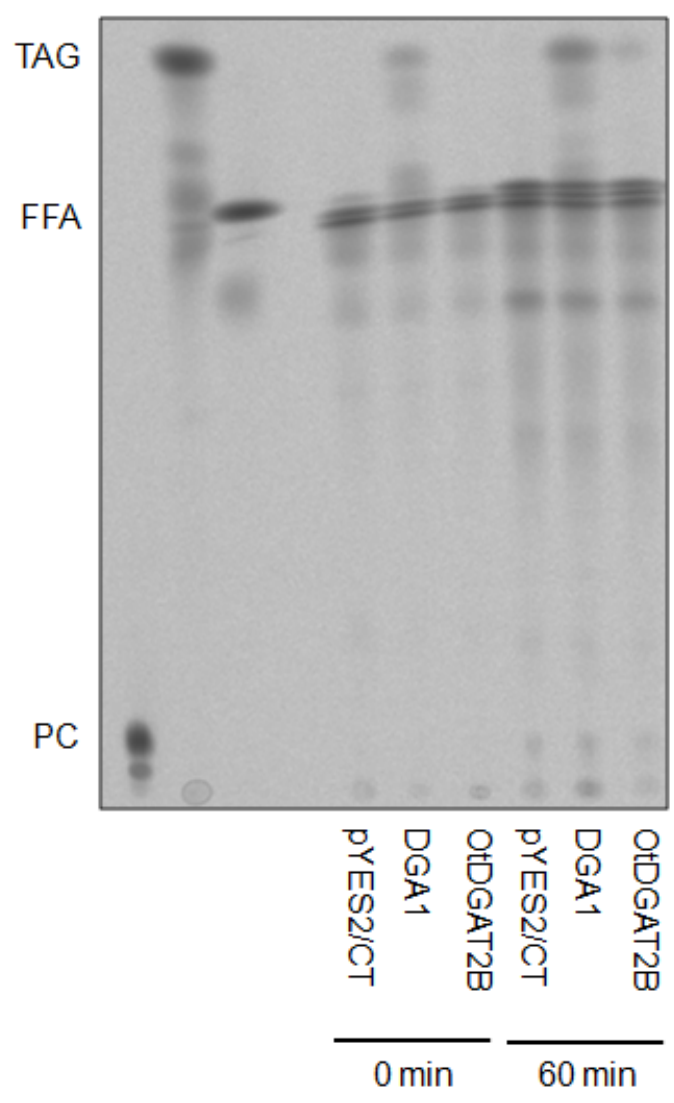

B

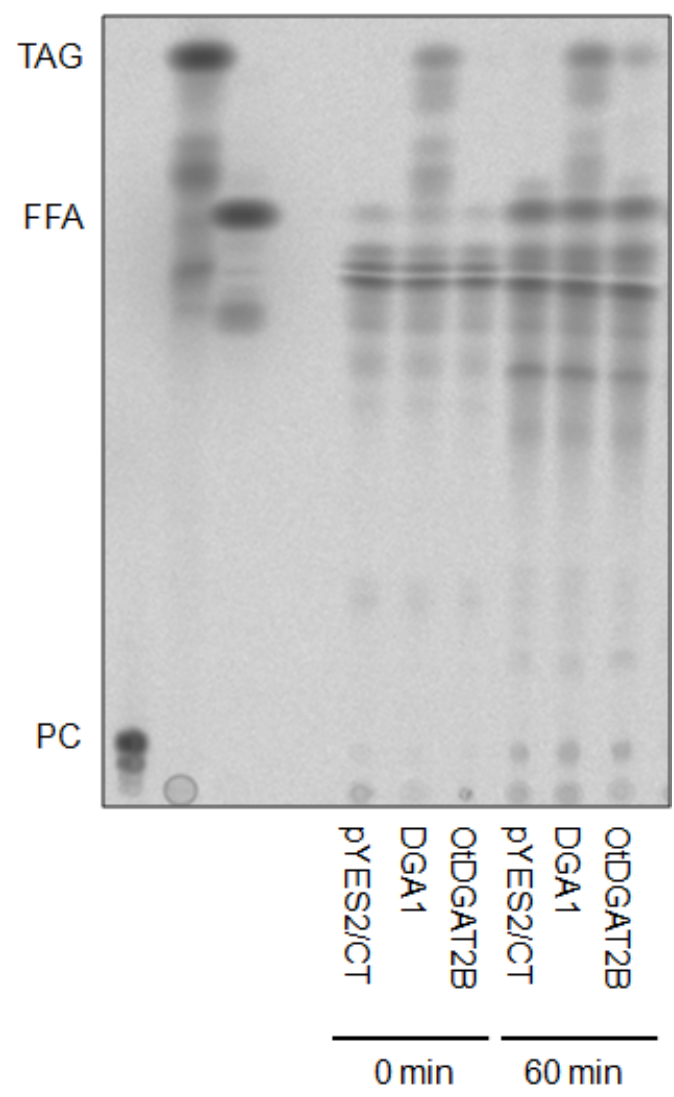

Figure 6. OtDGAT2Bp activity in vitro

pYES2/CT (negative control), DGA1-pYES2/CT (positive control) or OtDGAT2B-pYES2/CT were expressed for one day at $30{ }^{\circ} \mathrm{C}$ in the yeast mutant BY4741 dga1 $\Delta$ Iro1 $\Delta$ tes $1 \Delta$. Cell homogenates were prepared and incubated with $\left[1{ }^{14} \mathrm{C}\right]-18: 1(\mathrm{n}-9)-\mathrm{CoA}$ as acyl donor and $(\mathrm{A}) \mathrm{di}-$ 18:1(n-9)-DAG or (B) di-18:2(n-6)-DAG as acyl acceptors. Reactions were stopped at time points indicated. PC, phosphatidylcholine; TAG, triacylglycerol; FFA, free fatty acids. Presented is a representative result of $(\mathrm{A})$ two or $(\mathrm{B})$ one experiment(s).

\subsubsection{In vivo and in vitro studies of the putative acyl-CoA:diacylglycerol acyltransferases OtDGAT2Ap and OtDGAT2Cp}

OtDGAT2A and OtDGAT2C were identified in the same screen that also led to the identification of OtDGAT2B. Both sequences had to be completely codon-optimized to obtain detectable levels of protein after over-expression in yeast. In previous experiments OtDGAT2Ap and OtDGAT2Cp were not found to complement the TAG deficient yeast mutant BY4741 dga1 Iro1 $\Delta$ upon expression (Wagner, 2008). Because marine microalgae are adapted to life in the ocean, their enzymes might have different temperature optima compared for example to the corresponding yeast enzymes. Therefore, it the impact of expression temperature was tested. pYES2/CT-constructs containing either the completely codon-optimized nucleotide sequences of OtDGAT2A or Ot$D G A T 2 C$ or, as positive control, the partly codon-optimized sequence of OtDGAT2B under control of the GAL1 promoter (provided by Dr. M. Wagner) were transformed into 
BY4741 dga1 Iro1 $\Delta$. As negative control, the empty vector pYES2/CT was transformed into the same strain. Expression cultures were grown for 29 hours at $30{ }^{\circ} \mathrm{C}$ or for 95 hours at $23^{\circ} \mathrm{C}$ or $16{ }^{\circ} \mathrm{C}$. 24 OD units of each culture were harvested, total lipids were extracted and subsequently separated by TLC. The results of the performed experiment are depicted in Figure 7. Cultures expressing the empty vector pYES2/CT did not show a spot corresponding to TAG. OtDGAT2B-expressing cultures produced comparably high TAG amounts under all tested expression temperatures confirming the DGAT activity of OtDGAT2Bp. In contrast, cultures expressing OtDGAT2A produced very low amounts of TAG only at $23^{\circ} \mathrm{C}$ or even at $16{ }^{\circ} \mathrm{C}$. Cultures expressing OtDGAT2C did not produce TAGs under any of the given conditions.

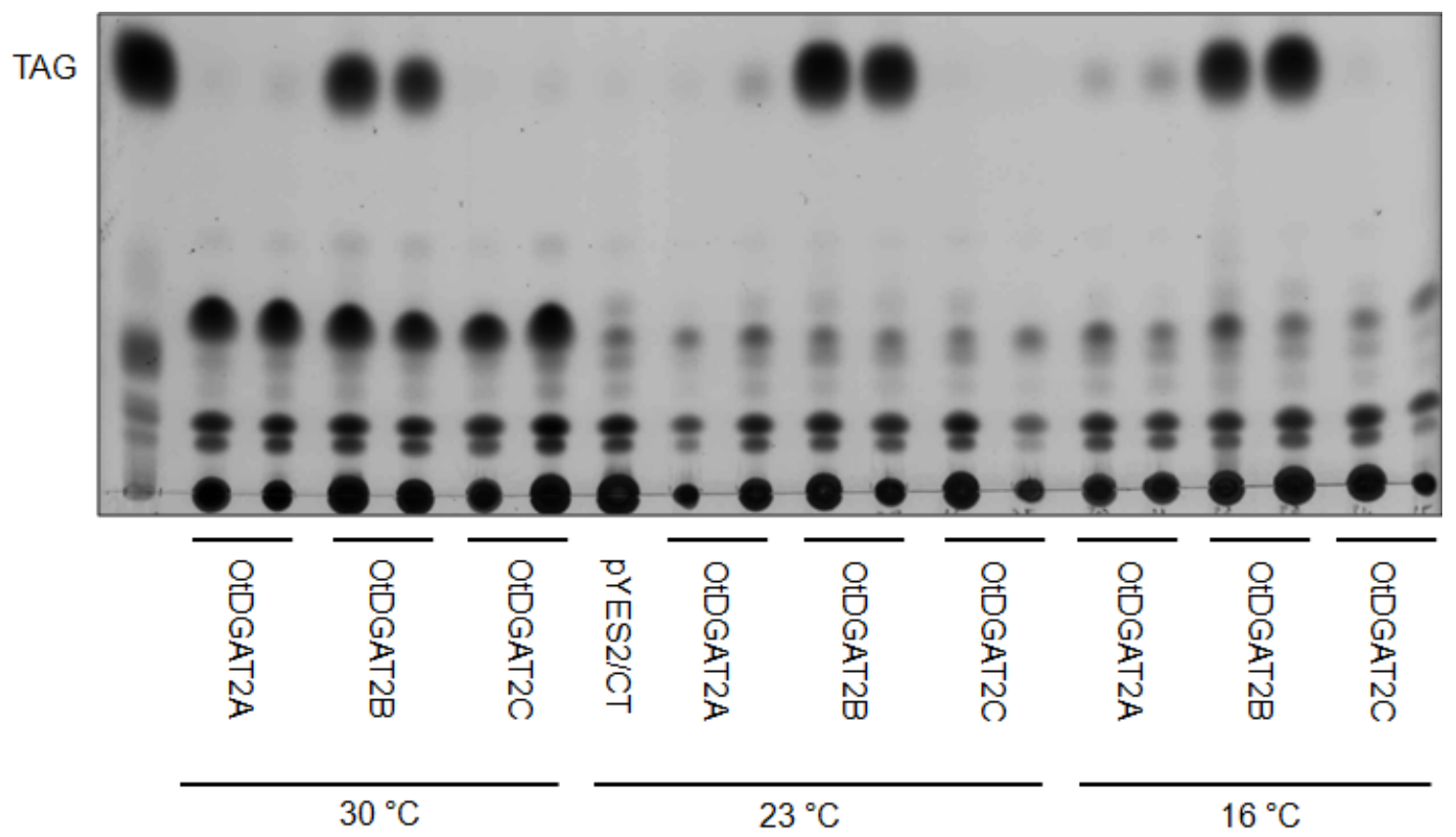

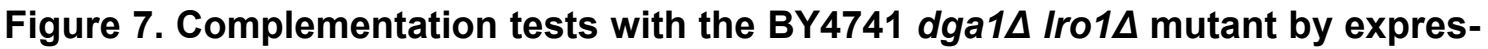
sion of OtDGAT2A, OtDGAT2B or OtDGAT2C

pYES2/CT (negative control), OtDGAT2A-pYES2/CT, OtDGAT2B-pYES2/CT (positive control) or OtDGAT2C-pYES2/CT were expressed at three different temperatures as indicated. Cultures were grown either for 29 hours at $30^{\circ} \mathrm{C}$ or for 95 hours at $23^{\circ} \mathrm{C}$ or $16{ }^{\circ} \mathrm{C}$, respectively. $24 \mathrm{OD}$ units were harvested, total lipids were extracted and separated by TLC. TAG, triacylglycerol. The experiment was performed once with two independent clones.

Bifunctional enzymes possessing DGAT and WS activity could be identified in bacteria (Kalscheuer and Steinbüchel, 2003) and plants (King et al., 2007; Li et al., 2007). In order to test whether OtDGAT2Ap or OtDGAT2Cp possess WS activity, their codonoptimized sequences were cloned into mCherryFAR1-pESC-URA (provided by Dr. M. Heilmann) downstream of the GAL10 promoter. The mouse fatty acid reductase (FAR1p) has been shown to reduce fatty acids bound to CoA into fatty alcohols (Cheng and Russell, 2004a). Mouse WS (provided by Dr. M. Heilmann) was used in the following experiment as positive control (Cheng and Russell, 2004b). Constructs harbouring $m$ CherryFAR1 alone as negative control or in combination with mouse WS or Ot- 
DGAT2A or OtDGAT2C, respectively, were transformed into the H1246 strain (by courtesy of Prof. Dr. S. Stymne) deficient in the TAG-producing enzymes DGA1p and LRO1p (Oelkers et al., 2000) and also lacking acyl-CoA:sterol acyltransferases ARE1p and ARE2p (Yang et al., 1996; Yu et al., 1996; Zweytick et al., 2000; Valachovič et al., 2001). This strain was used because of its lack of TAG and steryl esters (Sandager et al., 2002) and thus does not interfere with the assay. Expression cultures were either grown for 46 hours at $30{ }^{\circ} \mathrm{C}$ or $23{ }^{\circ} \mathrm{C}$ or for 116 hours at $16{ }^{\circ} \mathrm{C} .34 \mathrm{OD}$ units of each culture were harvested, total lipids were extracted and subsequently separated by TLC. The results of the performed experiment are depicted in Figure 8. Whereas the positive control shows wax ester accumulation for all expression conditions, those cultures coexpressing OtDGAT2A or OtDGAT2C together with FAR1 did not reveal any wax esters in their total lipids.

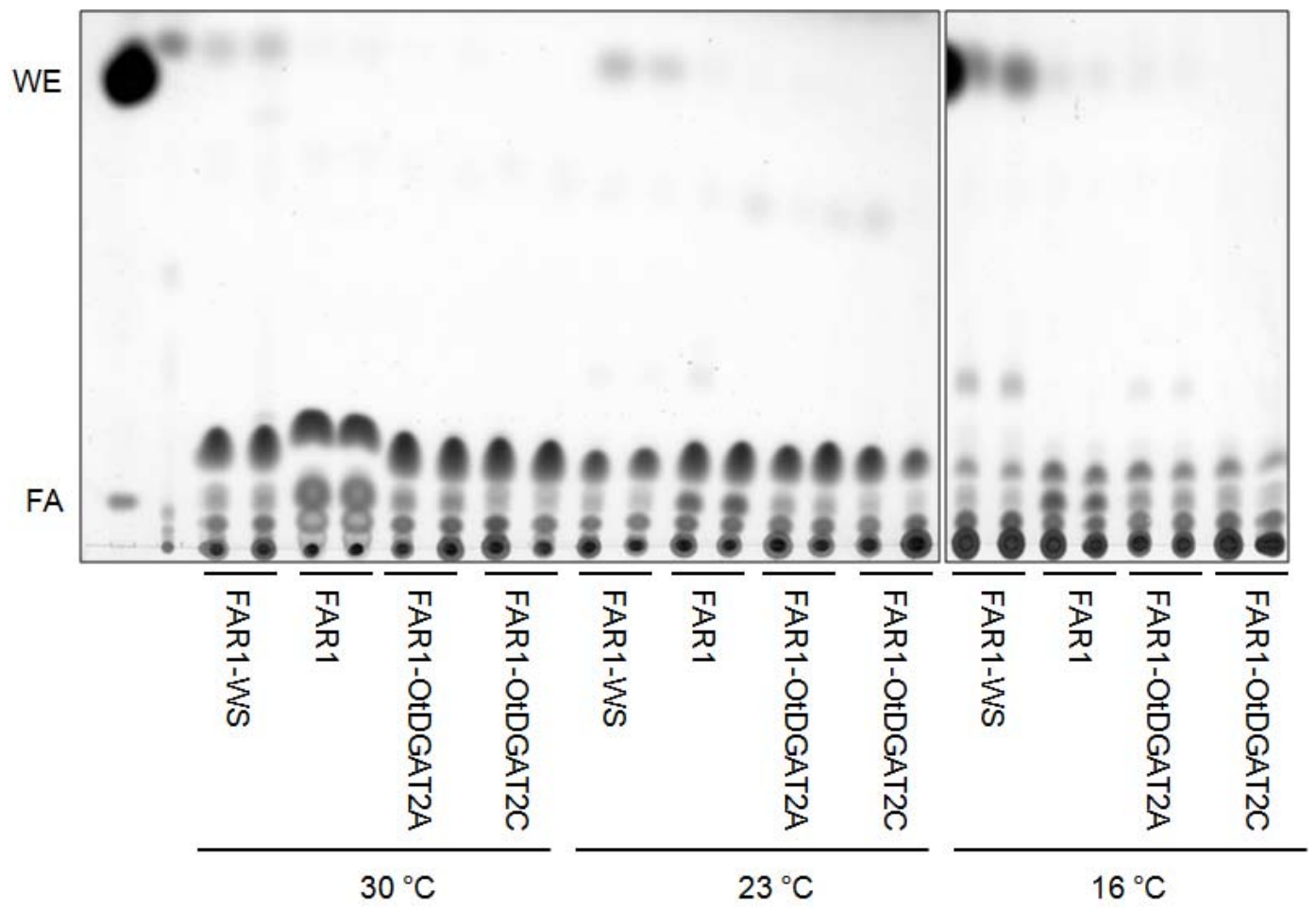

Figure 8. WS test in yeast strain $\mathrm{H} 1246$ by co-expression of mouse FAR1 with OtDGAT2A, OtDGAT2C or mouse WS

mCherryFAR1-pESC-URA (negative control), mCherryFAR1-OtDGAT2A-pESC-URA, mCherryFAR1-OtDGAT2C-pESC-URA or mCherryFAR1-WS-pESC-URA (positive control) were expressed at three different temperatures as indicated. Cultures were grown for 46 hours at $30^{\circ} \mathrm{C}$ or $23{ }^{\circ} \mathrm{C}$ or for 116 hours at $16{ }^{\circ} \mathrm{C} .34 \mathrm{OD}$ units of each culture were harvested, total lipids were extracted and subsequently separated by TLC. WE, wax esters; FA, fatty alcohols. The experiment was performed once with two independent clones. 
In order to test whether OtDGAT2Ap and OtDGAT2Cp revealed enzymatic activity in vitro, experiments similar to the assays performed for OtDGAT2Bp were conducted. pYES2/CT-constructs containing either codon-optimized OtDGAT2A, OtDGAT2C or, as positive control, the sequence of $D G A 1$ under control of the GAL1 promoter were trans-

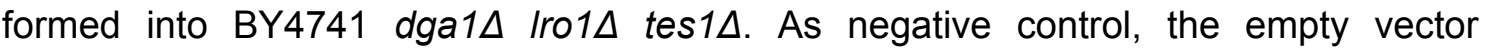
pYES2/CT was transformed into the same strain. Expression cultures were grown and homogenates were prepared as described above. The assay was performed with di18:1(n-9)-DAG as acyl acceptor and $\left[1-{ }^{14} \mathrm{C}\right]-18: 1(\mathrm{n}-9)-\mathrm{CoA}$ or $\left[1-{ }^{14} \mathrm{C}\right]-20: 4(\mathrm{n}-6)-\mathrm{CoA}$ as acyl donor. Results of the experiment are depicted in Figure 9. For homogenates from cultures expressing DGA1, TAG formation independent of the acyl donor could be observed. In contrast, homogenates from cultures expressing OtDGAT2A and OtDGAT2C did not show any DGAT activity in the assay.

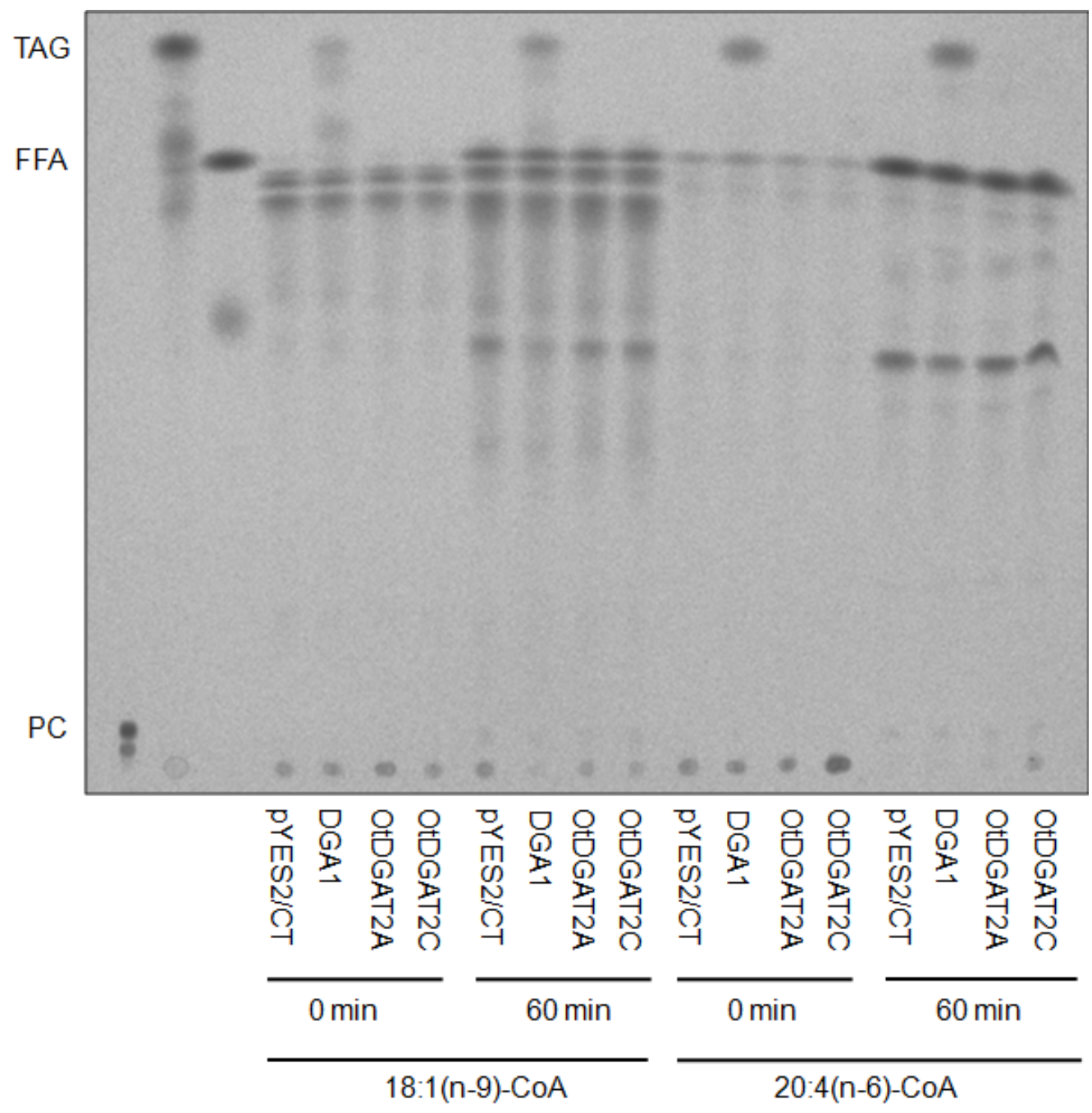

Figure 9. OtDGAT2Ap and OtDGAT2Cp activity in vitro

pYES2/CT (negative control), DGA1-pYES2/CT (positive control), OtDGAT2A-pYES2/CT or OtDGAT2C-pYES2/CT were expressed for one day at $30{ }^{\circ} \mathrm{C}$ in the yeast mutant BY4741 dga1 $\Delta$ Iro1 $\Delta$ tes $1 \Delta$. Cell homogenates were prepared and incubated with $\left[1-{ }^{14} \mathrm{C}\right]-18: 1(\mathrm{n}-9)-\mathrm{CoA}$ or $[1-$ $\left.{ }^{14} \mathrm{C}\right]-20: 4(\mathrm{n}-6)-\mathrm{CoA}$ and di-18:1(n-9)-DAG. Reactions were stopped at time points indicated. PC, 
phosphatidylcholine; TAG, triacylglycerol; FFA, free fatty acids. Presented is the result of one experiment.

Because OtDGAT2Ap and OtDGAT2Cp share sequence similarities not only with plant and animal DGATs, but also with animal MGATs (Wagner, 2008), it was tested as well whether the microalgal proteins exhibit MGAT activity in vitro. For this purpose, the assay was performed as described above, but under utilization of 18:1(n-9)-MAG instead of di-18:1(n-9)-DAG as acyl acceptor. As putative acyl donors, again $\left[1-{ }^{14} \mathrm{C}\right]-$ 18:1(n-9)-CoA and $\left[1-{ }^{14} \mathrm{C}\right]-20: 4(n-6)-C o A$ were used. For both enzymes, however, MGAT activity was not detectable (data not shown).

In summary, OtDGAT2A expression was able to complement a TAG-deficient yeast mutant under low expression temperatures in vivo. For OtDGAT2Cp, TAG complementation was not observable for any of the tested temperatures. Co-expression studies with both nucleotide sequences did not reveal WS activity and assays with radiolabeled substrates did not show DGAT or MGAT activity of the microalgal enzymes in vitro.

\subsubsection{Co-expression studies of different microalgal acyltransferase sequences}

The microalgal enzymes were further investigated by co-expressing different combinations in yeast to find out whether the acyltransferases possibly interact with each other or even are only active within a complex of different acyltransferases. This was tested by analyzing total amounts and fatty acid composition of different lipid classes and by comparison of the obtained data with those from cultures expressing one acyltransferase individually. Co-localization could be shown for example for the desaturase SCD1p and the acyltransferase DGAT2p from mouse (Man et al., 2006a). LPCAT in turn might potentially interact with both DGAT and PDAT enzymes because it mediates the transfer between acyl-CoA pool on the one hand and PC pool on the other hand. Therefore, combinations of LPCATp with PDATp or DGATp were chosen for the following experiments. For co-expression with OtLPCAT and OILPCAT, the sequence encoding the most active DGAT protein from $O$. tauri, OtDGAT2B, was chosen. In addition, OtPDAT from $O$. tauri was utilized. The nucleotide sequence was isolated by $\mathrm{Dr}$. $\mathrm{M}$. Wagner after searching through the genome of $O$. tauri with known PDAT sequences from yeast and Arabidopsis. Expression of a partly codon-optimized version of OtPDAT resulted in solid protein amounts in yeast. The enzyme was shown to complement the BY4741 Iro1 $\Delta$ dga1 $\Delta$ mutant only after addition of (n-3)- and (n-6)-polyunsaturated fatty acids with 18 or 20 carbon atoms (Wagner, 2008).

The yeast mutant BY4741 Iro1 $\Delta$ dga1 $\Delta$ ale1 $\Delta$ was created to avoid interference of the endogenous yeast acyltransferases with those of microalgal origin. The completely codon-optimized OILPCAT and the partly codon-optimized OtLPCAT were cloned into pESC-LEU downstream of the GAL1 promoter. Subsequently, pESC-LEU, OILPCATpESC-LEU or OtLPCAT-pESC-LEU were co-transformed with OtDGAT2B-pYES2/CT, OtPDAT-pYES2/CT or pYES2/CT into the newly created yeast mutant strain. As wild type control, BY4741 was co-transformed with pESC-LEU and pYES2/CT. Expression 
cultures were grown for two days at $23^{\circ} \mathrm{C}$ and subsequently harvested. Half of each sample was used for acyl-CoA extraction and measurement whereas the other half was utilized for total lipid extraction and separation of TAG from PC. Internal standards were used for quantification. Results of the performed experiment are depicted in Figure 10, Figure 11 and Figure 12. Acyl-CoA amounts were higher in the mutant compared to the wild type control, but this effect was not statistically significant (Figure 10 A). In all mutant expression cultures, 16:1(n-9)-fractions were elevated two to three times and percentages of 18:0 and 18:1(n-9) were lowered in comparison to wild type (Figure $10 \mathrm{~B}$ ). TAGs were detectable only in those mutant cultures expressing OtDGAT2B (Figure $11 \mathrm{~A}$ ), but the fatty acid composition of the TAG fraction from complemented mutant yeast cultures did not differ from wild type (Figure $11 \mathrm{~B}$ ). In case of OtPDAT expression, TAG levels were not significantly higher than in the negative control, the yeast mutant strain co-expressing pESC-LEU and pYES2/CT (Figure $11 \mathrm{~A}$ ). Low TAG levels in these cases did not lead to reliable data concerning fatty acid composition because of detection limitations in GC measurements. PC amounts were higher in the mutant yeast strain but this effect again was not statistically significant, as can be seen in Figure 12 A. Fatty acid composition of PC was nearly the same in all tested expression cultures (Figure $12 \mathrm{~B}$ ).

Generally it should be noted, that strong differences in total amounts as well as in fatty acid composition could be observed already between cultures co-expressing the same combination of acyltransferase nucleotide sequences. Therefore, it might be problematic to come to a conclusion based on the presented data. 
A

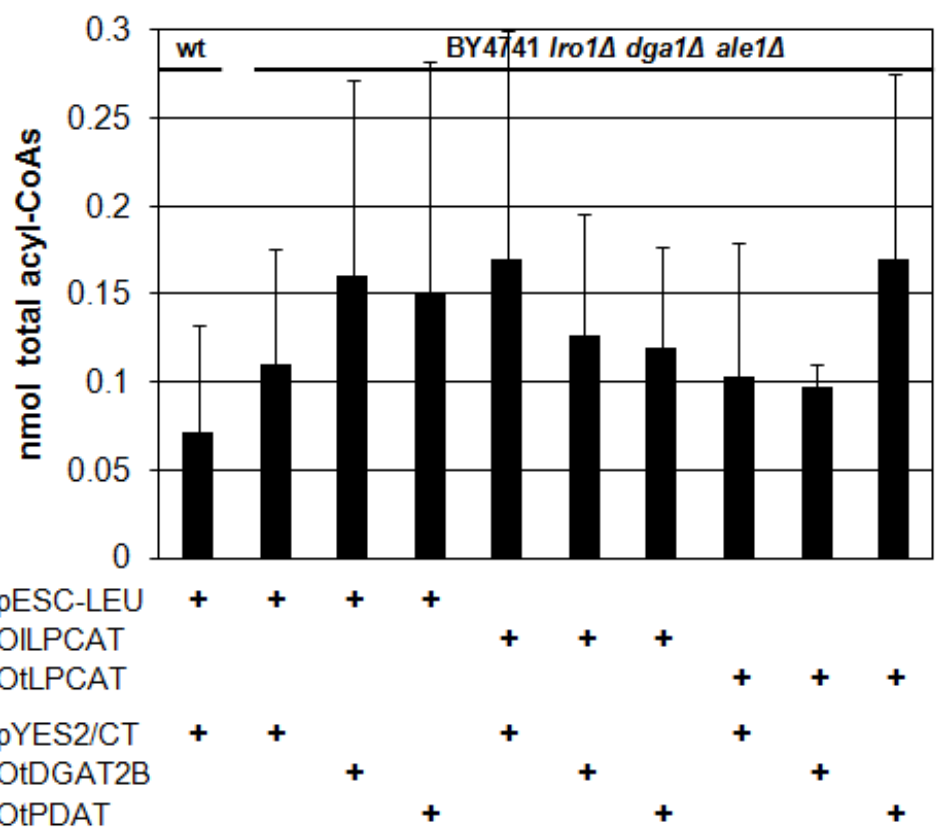

B

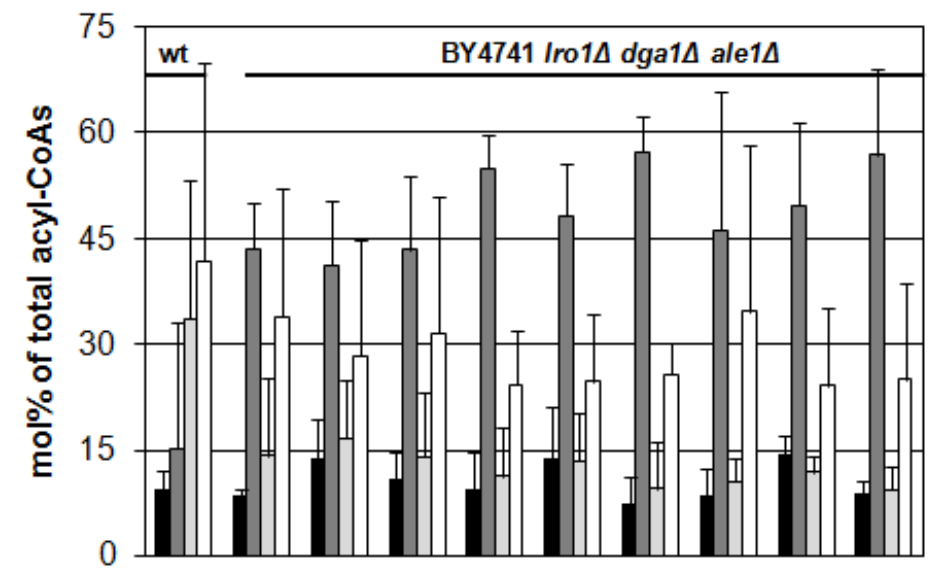

Figure 10. Acyl-CoAs after co-expression of microalgal acyltransferases in

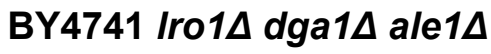

pESC-LEU, OILPCAT-pESC-LEU or OtLPCAT-pESC-LEU were co-expressed with pYES2/CT, OtDGAT2B-pYES2/CT or OtPDAT-pYES2/CT as indicated between the diagrams for two days at $23{ }^{\circ} \mathrm{C}$ in the yeast mutant strain or in BY4741 (wt). Yeast cells were harvested, acyl-CoAs were extracted and measured. (A) total amounts and (B) mol\% of different acyl chains. AcylCoA, acyl-Coenzyme A. Bars represent the mean + standard deviation (SD) of three to four independent clones. 
A

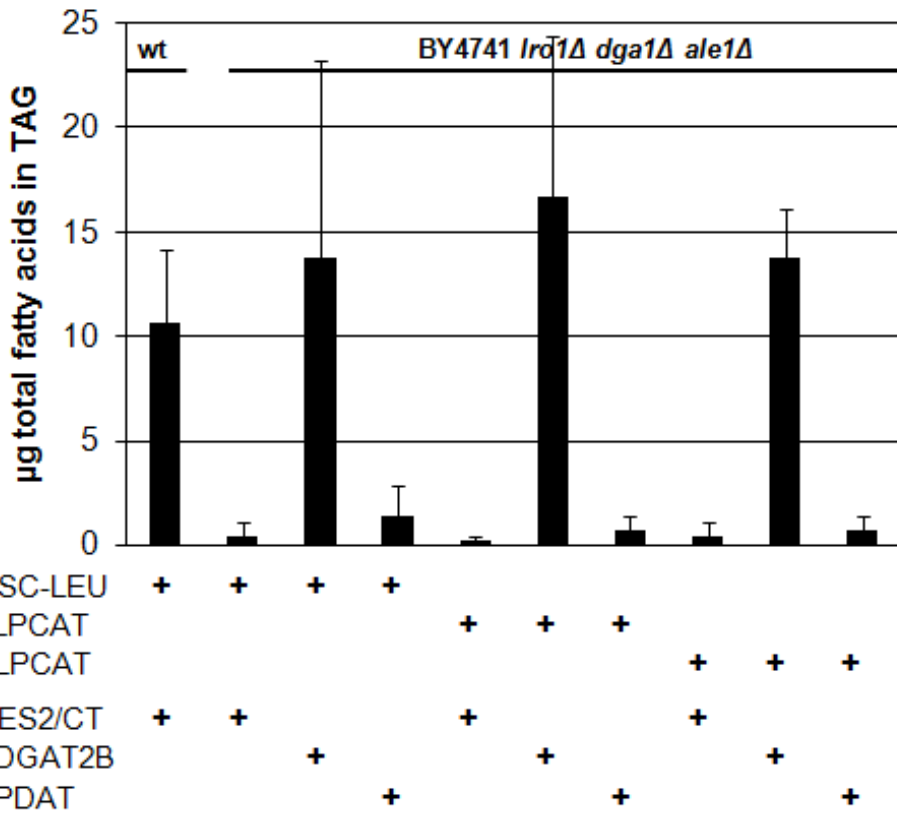

B

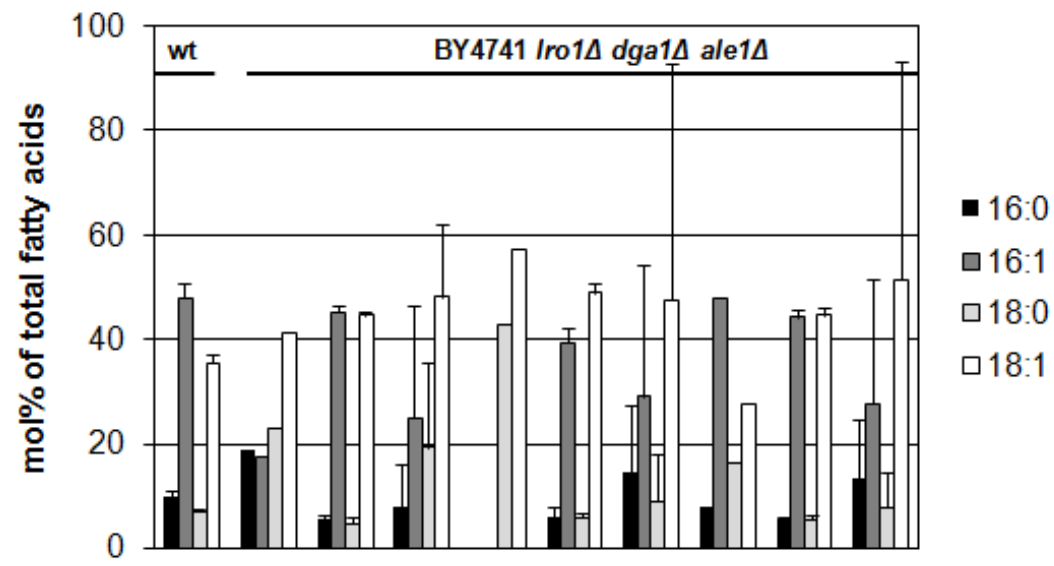

Figure 11. TAG after co-expression of microalgal acyltransferases in BY4741

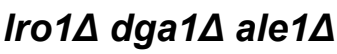

pESC-LEU, OILPCAT-pESC-LEU or OtLPCAT-pESC-LEU were co-expressed with pYES2/CT, OtDGAT2B-pYES2/CT or OtPDAT-pYES2/CT as indicated between the diagrams for two days at $23{ }^{\circ} \mathrm{C}$ in the yeast mutant strain or in BY4741 (wt). Yeast cells were harvested, total lipids were extracted and TAGs were analyzed. (A) total amounts and (B) mol\% of different acyl chains. TAG, triacylglycerol. Bars represent the mean $+S D$ of three to four independent clones. 
A

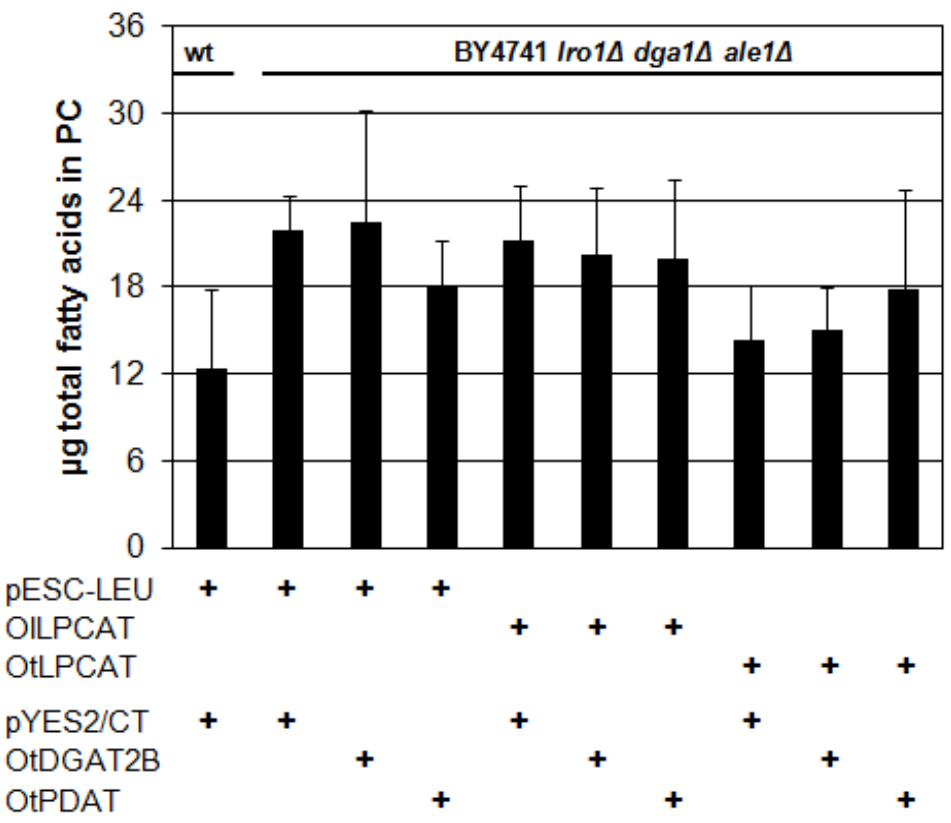

B

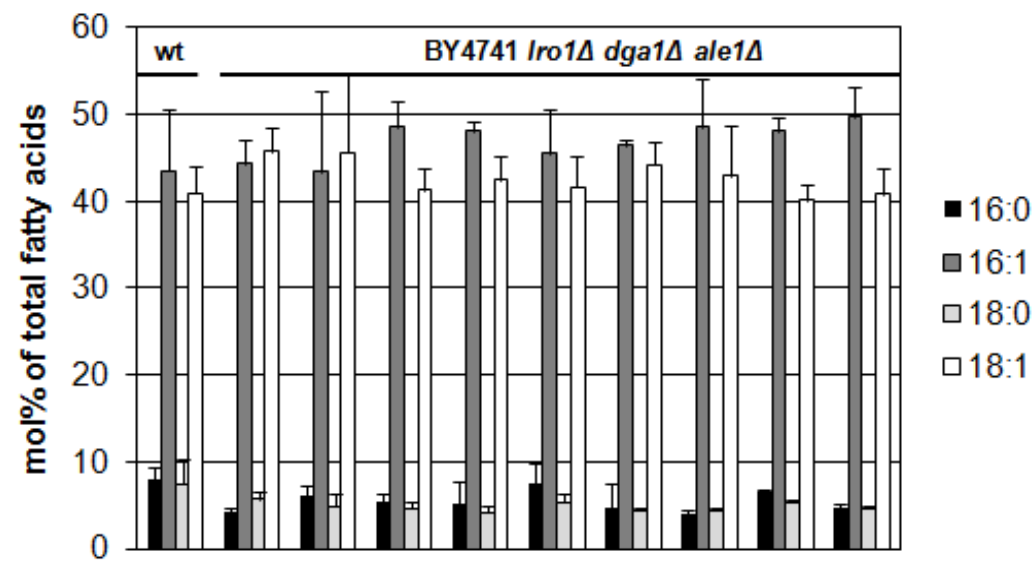

Figure 12. PC after co-expression of microalgal acyltransferases in BY4741 Iro1A

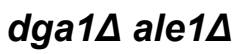

pESC-LEU, OILPCAT-pESC-LEU or OtLPCAT-pESC-LEU were co-expressed with pYES2/CT, OtDGAT2B-pYES2/CT or OtPDAT-pYES2/CT as indicated between the diagrams for two days at $23{ }^{\circ} \mathrm{C}$ in the yeast mutant strain or in BY4741 (wt). Yeast cells were harvested, total lipids were extracted and PC was analyzed. (A) total amounts and (B) mol\% of different acyl chains. PC, phosphatidylcholine. Bars represent the mean + SD of three to four independent clones. 


\subsection{Characterization of the microalgal desaturase Old4p in yeast}

In previous studies, acyl-CoA-dependent front-end desaturases with $\Delta 6$ - and $\Delta 5$ specificity were identified from O. tauri and M. squamata (Domergue et al., 2005; Hoffmann et al., 2008). Their corresponding cDNA sequences were tested in planta and led, upon co-expression with the elongase sequence PSE1 (Zank et al., 2002), to EPA production in Arabidopsis seeds. In order to establish exclusively CoA-dependent DHA synthesis, the additional implementation of an acyl-CoA-dependent desaturase with $\Delta 4$-specificity as well as an elongase with $\Delta 5$-specificity would be required. Because $\Delta 5$-elongases always act in an acyl-CoA-dependent way (Domergue et al., 2003) and have already been isolated from a few microalgae (Meyer et al., 2004; Pereira et al., $2004 \mathrm{~b}$ ), the focus was put on the identification of a $\Delta 4$-desaturase. O. lucimarinus in this respect appeared as a promising gene donor because it is closely related to $O$. tauri and M. squamata and therefore might also possess acyl-CoA-dependent desaturases.

\subsubsection{Investigation of the potential gene donor 0 . lucimarinus}

In order to test whether $O$. lucimarinus would be a useful candidate for isolation of a $\Delta 4$-desaturase encoding cDNA, the fatty acid composition of total lipids from 0 . lucimarinus cultures was examined first. Fatty acid profile analysis revealed sound amounts of 16:4(n-3) and DHA (Figure 13). 16:4(n-3) accounted for $14 \%$ of total fatty acids, DHA amounts were with $3 \%$ less pronounced. Another remarkable observation was the predominant accumulation of VLCPUFAs from the (n-3)-series that was also observable for M. squamata and $O$. tauri (Hoffmann, 2008). The fatty acid profile of $O$. lucimarinus thus showed several precursors of DHA which indicates that VLCPUFA synthesis in this organism occurs via sequential desaturation and elongation, but not via a polyketide synthase-like system which is characterized by releasing only trace amounts of intermediates (Truksa et al., 2009). Taken together, these results suggested $O$. Iucimarinus as suitable gene donor for a $\Delta 4$-desaturase sequence. 


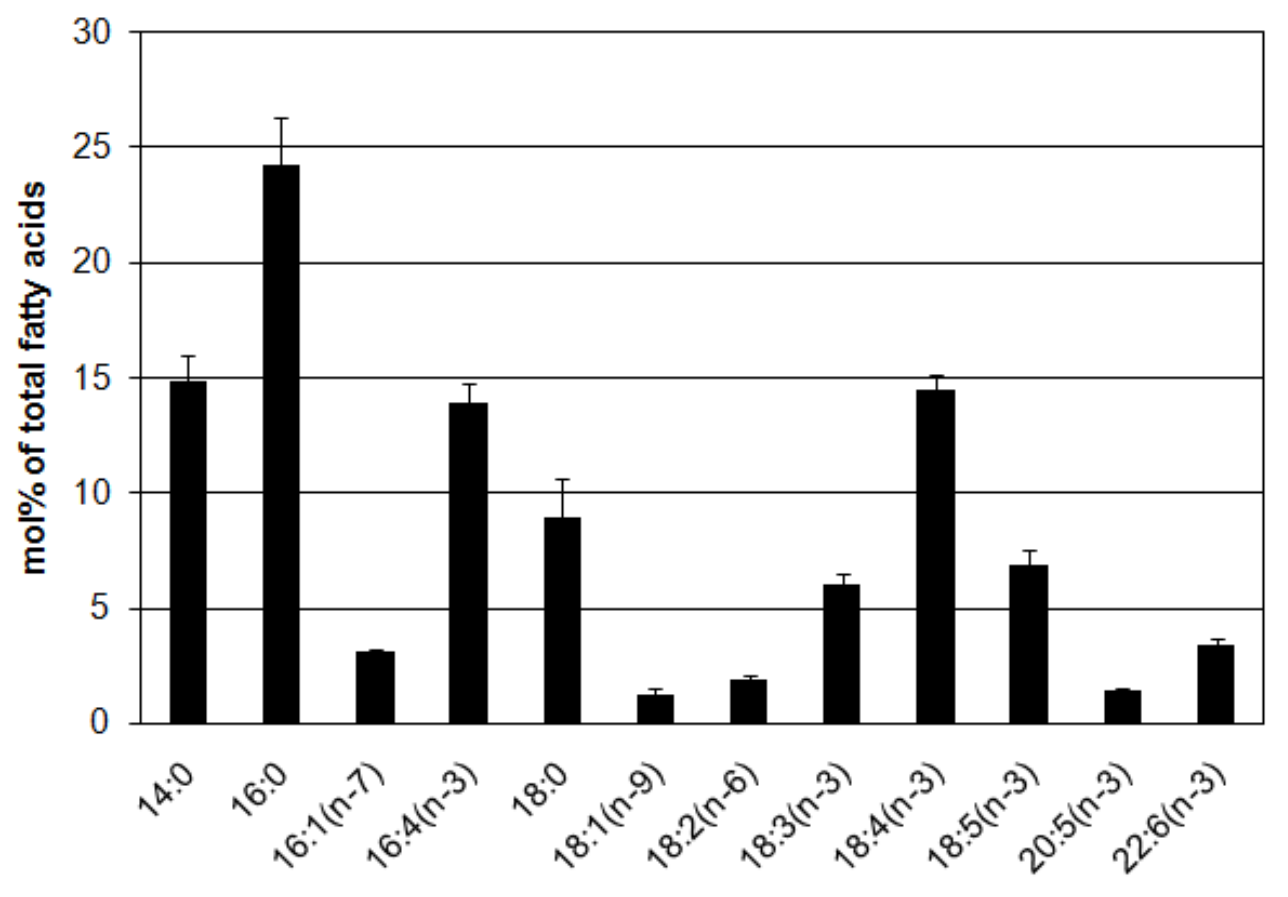

Figure 13. Fatty acid composition of total lipid extracts from 0 . Iucimarinus

Cells were harvested from liquid cultures and total lipids were extracted. Fatty acids were derivatized into their respective methyl esters. These were analyzed and identified via GC-FID and GC-MS. Bars represent the mean + SD of five independent samples.

\subsubsection{Identification of a putative front-end desaturase with $\Delta 4$-specificity}

The microalgal genome, which had been sequenced recently by (Palenik et al., 2007), was scanned via BLAST search with already known front-end desaturases as queries for new putative desaturase sequences (Altschul et al., 1997). The identified nucleotide sequence (GenBank accession number XM_001415706) encoded a polypeptide of 459 amino acids (GenBank accession number XP_001415743) which was then further analyzed by similarity searches in the NCBI database using the BLAST program. Hits with experimentally proven substrate specificity were chosen for global pairwise alignment with the putative protein sequence Old4p. Old4p showed highest identity to two $\Delta 4$ desaturases from Thraustochytrium sp. (Qiu et al., 2001; Liu et al., 2007) with identity values of $28 \%$ and $28.2 \%$, respectively, in pairwise alignment (Table 2). Also, $\Delta 4-$ desaturases from E. gracilis (Meyer et al., 2003) and T. pseudonana (Tonon et al., $2005 \mathrm{~b}$ ) shared high identity values of $25.8 \%$ and $25.3 \%$, respectively, with Old4p. As can be seen from Table 2 , also several desaturases with $\Delta 5$ - or $\Delta 8$-specificity were found to be similar to Old4p. 
Table 2. Results from the pairwise alignment of Old4p with related proteins

Sequences are arranged according to their appearance in the BLAST search.

\begin{tabular}{|c|c|c|c|}
\hline Related protein & $\begin{array}{c}\text { Accession } \\
\text { number }\end{array}$ & $\begin{array}{c}\text { Identity } \\
(\%)\end{array}$ & $\begin{array}{c}\text { Similarity } \\
(\%)\end{array}$ \\
\hline $\begin{array}{l}\text { Thraustochytrium sp. } \Delta 4 \text {-desaturase } \\
\text { (Tsd4_1p) }\end{array}$ & AAM09688 & 28.2 & 42.9 \\
\hline $\begin{array}{l}\text { Thraustochytrium sp. } \Delta 4 \text {-desaturase } \\
\text { (Tsd4_2p) }\end{array}$ & AAZ43257 & 28 & 42.7 \\
\hline $\begin{array}{l}\text { Euglena gracilis } \Delta 4 \text {-desaturase } \\
\text { (Egd4p) }\end{array}$ & AAQ19605 & 25.8 & 38.5 \\
\hline $\begin{array}{l}\text { Thalassiosira pseudonana } \Delta 4 \text {-desaturase } \\
(\mathrm{Tpd} 4 \mathrm{p})\end{array}$ & AAX14506 & 25.3 & 39.9 \\
\hline $\begin{array}{l}\text { Oblongichytrium sp. } \Delta 5 \text {-desaturase } \\
\text { (Osd5p) }\end{array}$ & BAG71007 & 26.7 & 40.3 \\
\hline $\begin{array}{l}\text { Marchantia polymorpha } \Delta 5 \text {-desaturase } \\
\text { (Mpd5p) }\end{array}$ & AAT85663 & 25.7 & 42.9 \\
\hline $\begin{array}{l}\text { Mortierella alpina } \Delta 5 \text {-desaturase } \\
\text { (Mad5_1p) }\end{array}$ & BAD95486 & 26.8 & 40.2 \\
\hline $\begin{array}{l}\text { Mortierella alpina } \Delta 5 \text {-desaturase } \\
(\text { Mad5_2p) }\end{array}$ & AAC72755 & 26.6 & 40.1 \\
\hline $\begin{array}{l}\text { Thalassiosira pseudonana } \Delta 8 \text {-desaturase } \\
\text { (Tpd8p) }\end{array}$ & AX14502 & 25.6 & 42.3 \\
\hline $\begin{array}{l}\text { Mantoniella squamata } \Delta 5 \text {-desaturase } \\
\text { (Msd5p) }\end{array}$ & CAQ30478 & 26.8 & 41.6 \\
\hline $\begin{array}{l}\text { Phaeodactylum tricornutum } \Delta 5 \text {-desaturase } \\
\text { (Ptd5p) }\end{array}$ & AAL92562 & 27.7 & 41.8 \\
\hline $\begin{array}{l}\text { Pavlova lutheri } \Delta 4 \text {-desaturase } \\
\text { (Pld4p) }\end{array}$ & AAQ98793 & 25.7 & 39.9 \\
\hline
\end{tabular}


A phylogenetic tree of the chosen sequences was generated using the program Phylip (Figure 14). A multiple alignment was created with the $\Delta 4$-desaturases found via BLAST search using the online softwares MultAlin and Boxshade 3.21. In Figure 15, the multiple alignment of Old4p with its related sequences is depicted in extracts. Old $4 p$ has three histidine boxes that are necessary for coordination of two iron atoms as redox unit for the catalytical center of the desaturase (Shanklin et al., 1994; Shanklin and Cahoon, 1998). The histidine boxes (marked with continuous lines) have the sequences $\mathrm{HX}_{3} \mathrm{H}, \mathrm{HX}_{3} \mathrm{H}_{2}$ and $\mathrm{QX}{ }_{2} \mathrm{H}_{2}$. Hence, the last box shows an $\mathrm{H}$ to $\mathrm{Q}$ substitution typical for front-end desaturases (Sperling and Heinz, 2001). In addition to that, it contains an aminoterminal HPGG-motif (marked with a dashed line) which indicates a fused cytochrome $b_{5}$ domain also characteristic for microsomal desaturases (Sperling et al., 1995; Mitchell and Martin, 1997; Napier et al., 1997).

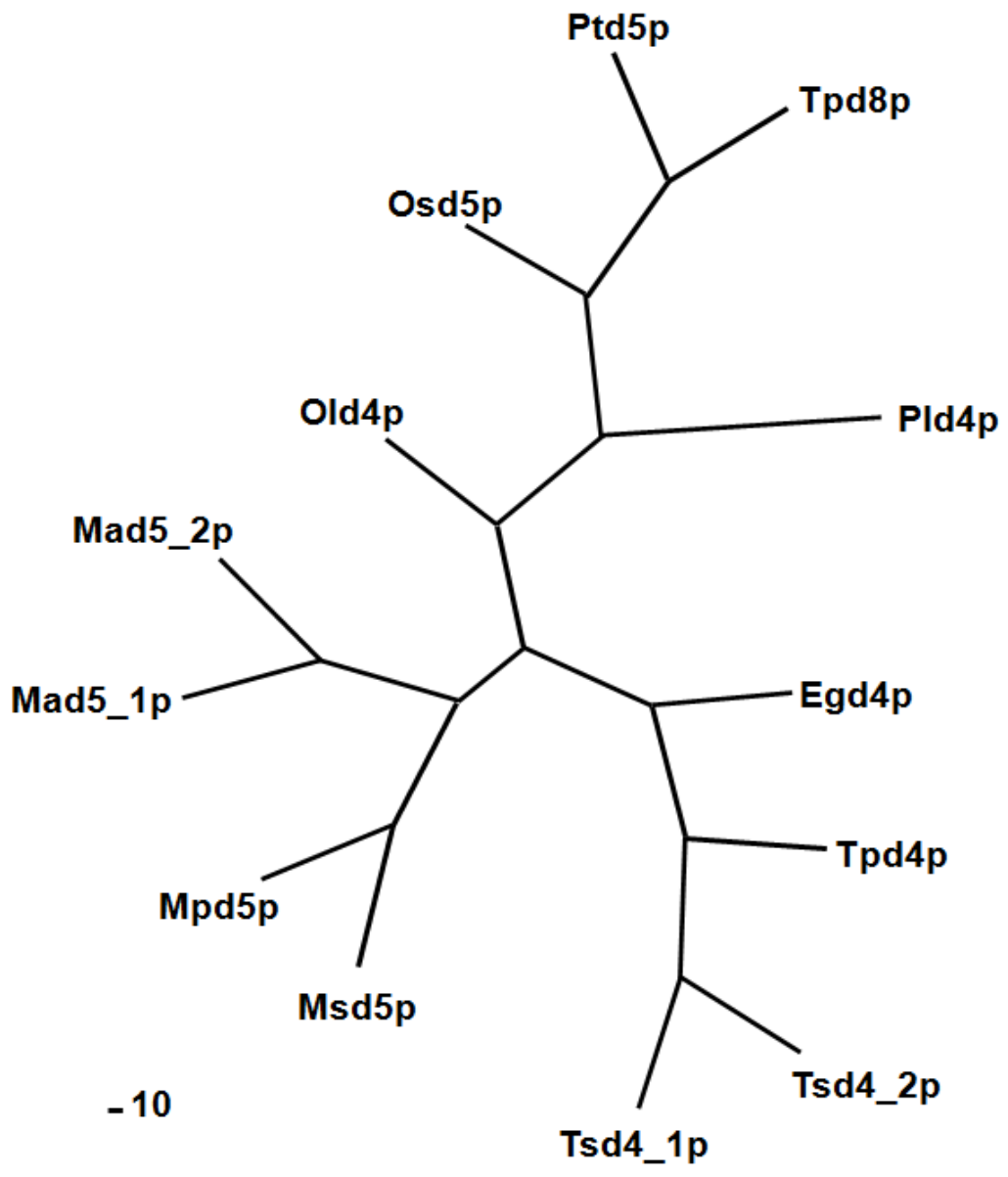

Figure 14. Phylogram of Old4p and selected functionally characterized $\Delta 4-, \Delta 5-$ and $\Delta 8$-desaturases

The phylogram was calculated based on aligned amino acid sequences using the CLUSTALX multiple alignment and the Phylip program. Details concerning GenBank accession numbers and organisms are given in Table 2. 


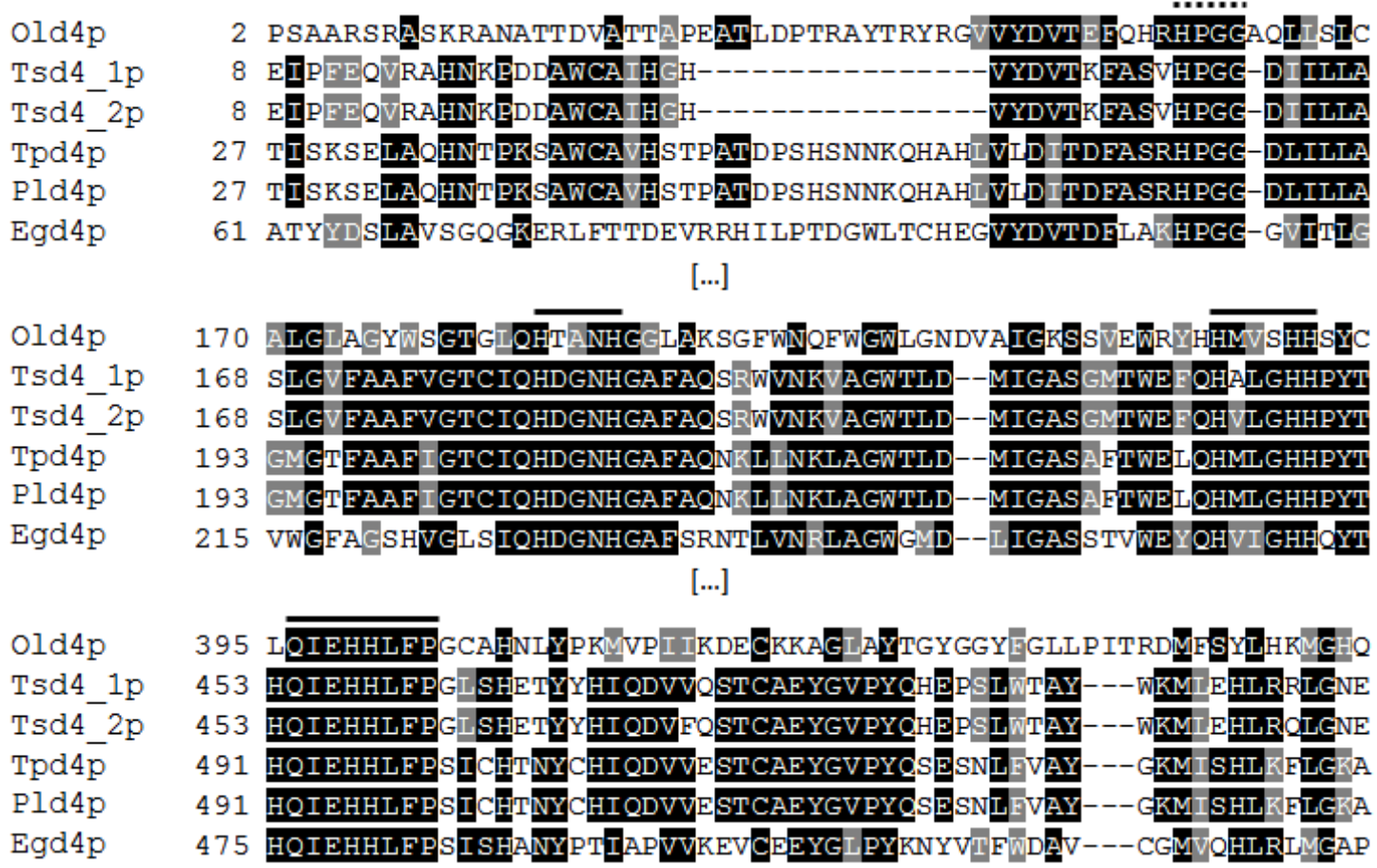

Figure 15. Partial alignment of the deduced amino acid sequence for Old4p with related $\Delta$ 4-desaturases

Identical residues are shaded in black, conserved residues are shaded in grey. The three histidine boxes are marked with a continuous line. The conserved amino acid residues of the cytochrome $b_{5}$ domain are marked with a dashed line. Details concerning GenBank accession numbers and organisms are given in Table 2.

\subsubsection{Catalytic activity and substrate specificities of the desaturase Old4p}

In order to investigate the enzymatic activity of Old4p, the full-length nucleotide sequence of Old4 was isolated from genomic DNA (provided by Dr. M. Heilmann), partly codon-optimized and cloned into the yeast expression vector pESC-TRP downstream of the galactose-inducible promoter GAL10. The resulting construct was transformed into the yeast strain INVSc1. As negative control, empty pESC-TRP vector was transformed into yeast. As positive control, the $\Delta 4$-desaturase nucleotide sequence Egd4 from E. gracilis (Meyer et al., 2003) was isolated (provided by S. Schlenczek), cloned into pESC-TRP and transformed into INVSc1. Yeast is approved as suitable expression host for desaturase substrate specificity tests (Michaelson et al., 1998; Domergue et al., 2002; Whitney et al., 2003). Although expression in yeast might not always resemble cofactor and lipid composition of the gene donor organism (Heilmann et al., 2004), it might still allow conclusions for enzyme function. Because yeast contains only a limited number of endogenous fatty acids, namely 16:0, 16:1(n-7), 18:0, 18:1(n-9) and 18:1(n-7), substrate specificity of hypothetical desaturases was tested by addition of different free fatty acids as substrates to yeast cultures. Expression cultures were grown for three days at $23{ }^{\circ} \mathrm{C}$. Afterwards, cultured cells were harvested, their fatty acids were derivatized into the respective FAMEs and analyzed by GC. Desaturation activity of Old4p was detected only in presence of $22: 4(n-6), 22: 5(n-3)$ and $16: 3(n-3)$. In 
Figure 16, an exemplary FAME profile of INVSc1 supplemented with 22:5(n-3) and expressing either the empty vector control, Old4 or Egd4 is depicted. Whereas in the control solely the supplemented substrate is observable, cultures expressing Old4 or Egd4 produce the desaturation product $22: 6(n-3)$. Product identity was verified by GCFID via coelution with standard fatty acids as well as by GC-MS.

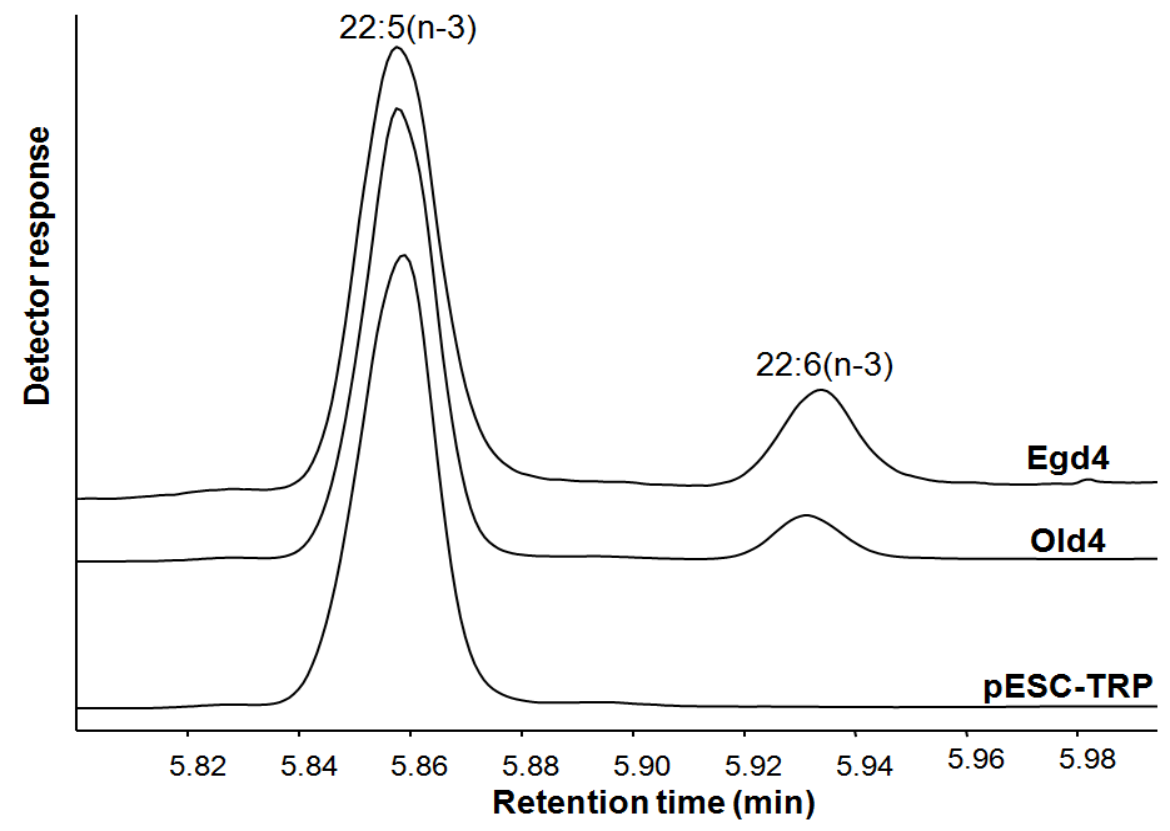

Figure 16. Gas chromatograms after expression of Old4 and Egd4 in yeast

FAMEs were prepared from INVSc1 cultures supplemented with $200 \mu \mathrm{M} 22: 5(\mathrm{n}-3)$ after expression of pESC-TRP (negative control), Old4-pESC-TRP (Old4) or Egd4-pESC-TRP (Egd4; positive control) for three days at $23^{\circ} \mathrm{C}$. Product identity was verified by GC-FID and by GC-MS. Representative results of three independent experiments are shown.

In Figure 17, desaturation efficiencies of cultures expressing Old4 or Egd4 are compared. In these experiments, the preferred substrate of Old4p with a desaturation activity of about $10 \%$ was $22: 5(n-3)$ followed by $22: 4(n-6)$ with an efficiency of about $4 \%$. $16: 3(n-3)$ was desaturated only very poorly with an efficiency of about $1 \%$. Egd $4 p$ showed the strongest activity of $15 \%$ on 16:3(n-3) and desaturated $22: 5(n-3)$ and $22: 4(n-6)$ with efficiencies of $12 \%$ and $4 \%$, respectively. It should be stated that the original version of Old4 upon expression in yeast did not lead to any desaturation products. The reason for this might be abortion of mRNA translation in yeast due to the lack of appropriate tRNAs. However, also expression of a completely codon-optimized synthetic nucleotide sequence encoding the same polypeptide as Old4 did not further enhance desaturation activity in yeast compared to the partly codon-optimized version. This finding shows that translation efficiency did not seem to be the only limiting factor for the microalgal desaturase activity in yeast.

In a nutshell, Old4p desaturated both (n-3)- and (n-6)-substrates. The enzyme furthermore preferred VLCPUFAs to a shorter-chain substrate. 


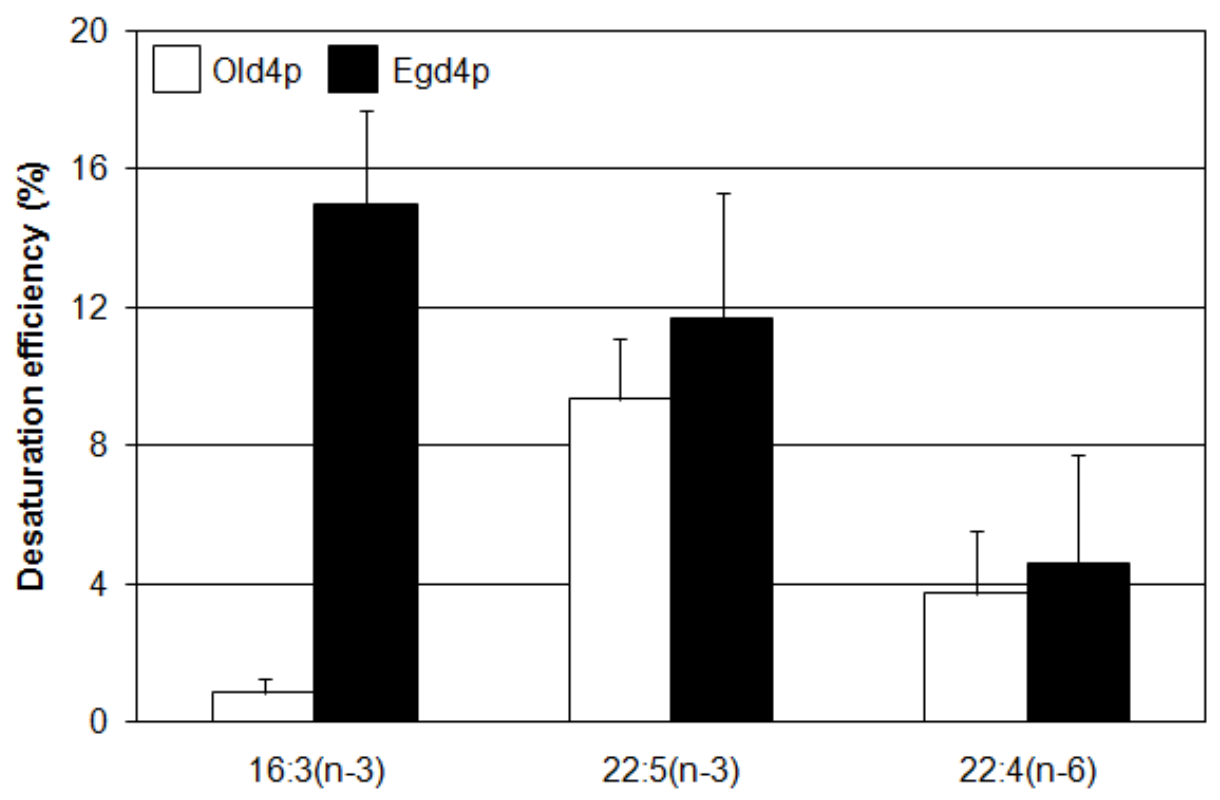

Figure 17. Conversion rates of supplied fatty acids by Old4p and Egd4p in yeast Old4-pESC-TRP or Egd4-pESC-TRP were co-expressed with pESC-LEU for three days at $23^{\circ} \mathrm{C}$ in INVSc1 cells supplemented with $200 \mu \mathrm{M}$ of the respective fatty acid. FAMEs from yeast cells were prepared and analyzed by GC-FID and GC-MS. Desaturation efficiency was calculated as $100^{*}$ product/(substrate + product). Bars represent the mean + SD of three independent experiments.

\subsubsection{Distribution of Old4p and Egd4p desaturation products in different yeast lipid classes}

From the distribution of desaturation product between the different lipid classes it can be deduced whether the tested desaturase acts in an acyl-CoA-dependent or in a lipiddependent manner (Domergue et al., 2003; Hoffmann et al., 2008). Therefore, the fatty acid composition of the different lipid classes from yeast cultures expressing Old4 or Egd4 was analyzed (Figure 18). In both cases, more than $50 \%$ of the desaturation product was present in the PC fraction of the cells. About $40 \%$ of product was found in the TAG fraction, and only trace amounts of DHA could be identified in the PE or PS and $\mathrm{PI}$ fractions. The latter two fractions were inseparable by the utilized fractionation method. Hence, they are depicted in a combined way. These results are similar to desaturation product distributions found for the lipid-dependent desaturases Ptd5p and Ptd6p (Domergue et al., 2003; Hoffmann et al., 2008). 


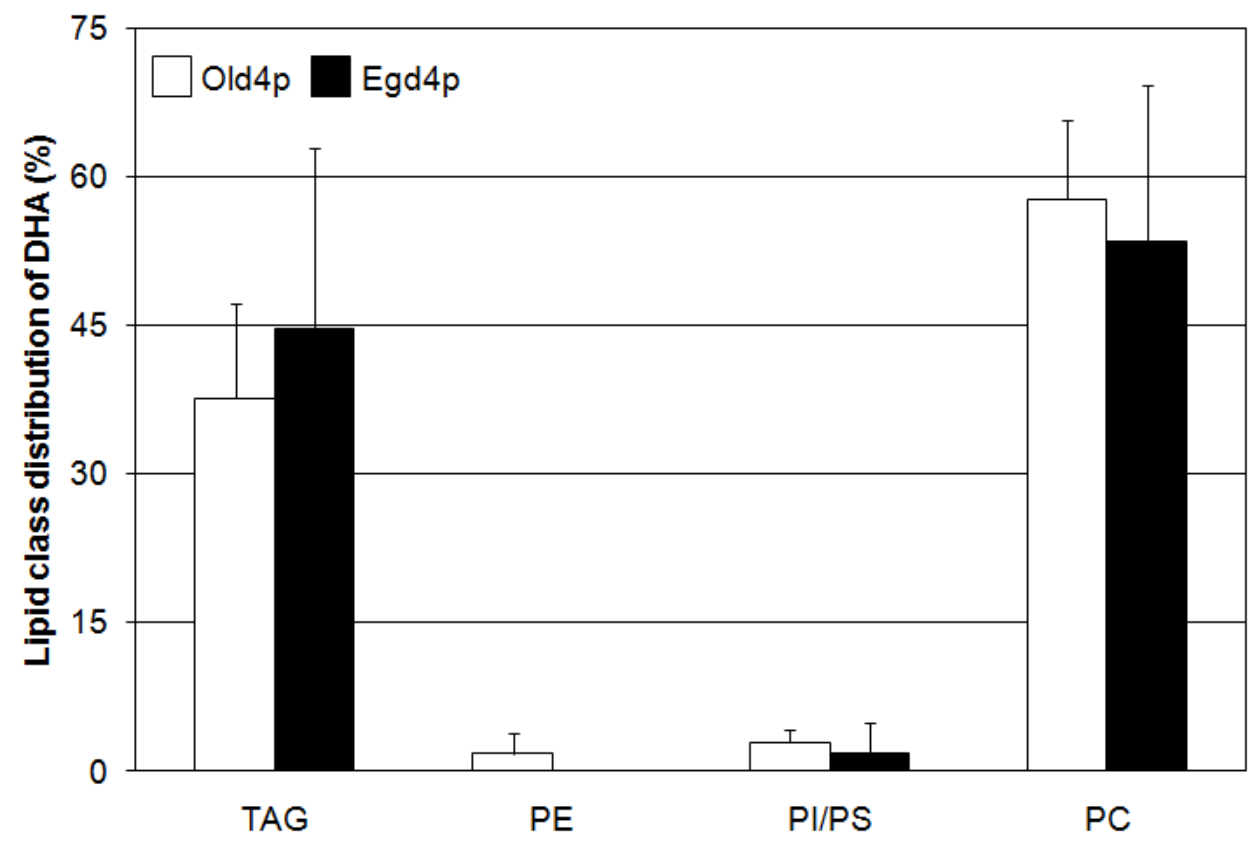

Figure 18. Lipid class distribution of DHA after action of Old $4 p$ or Egd4p in yeast Old4-pESC-TRP or Egd4-pESC-TRP were expressed for three days at $23^{\circ} \mathrm{C}$ in INVSc1 cells supplemented with $200 \mu \mathrm{M}$ 22:5(n-3). Total lipids were extracted from yeast cultures and separated by TLC. Then they were derivatized into their respective FAMEs and analyzed by GC. TAG, triacylglycerol; PE, phosphatidylethanolamine; PI, phosphatidylinositol; PS, phosphatidylserine; PC, phosphatidylcholine. Bars represent the mean + SD of three independent experiments.

Nevertheless, O. lucimarinus is a close relative of $O$. tauri and $M$. squamata, which both have been shown to contain acyl-CoA-dependent desaturases with $\Delta 5$ - and $\Delta 6$ specificity (Domergue et al., 2005; Hoffmann et al., 2008). To check whether the low desaturation activity of Old4p was caused by insufficient substrate amounts in the form of acyl-CoAs, co-expression experiments were performed with a long-chain acyl-CoA synthetase from T. pseudonana (TpLACSp) under addition of 22:5(n-3). TpLACS has been characterized by heterologous expression in yeast by (Tonon et al., 2005a). In these studies, the enzyme was shown to convert externally supplied VLCPUFAs into the respective CoA esters with DHA as the preferred substrate (Tonon et al., 2005a). Thus, the rationale behind this co-expression experiment was to enhance 22:5(n-3)CoA levels in yeast cells in order to test whether Old4p exhibits increased activity under these conditions. However, co-expression of Old4 with TpLACS did not result in a higher desaturation efficiency of Old4p, as can be seen in Figure 19. Whereas the desaturation efficiency upon co-expression of Old4 with pESC-LEU was $16 \%$ in the experiment performed, co-expression with TpLACS led to a lower conversion rate of about $9 \%$. It could also be observed that the total amount of 22:5(n-3) in TpLACSexpressing cultures was not significantly higher compared to cultures expressing the empty vector. 


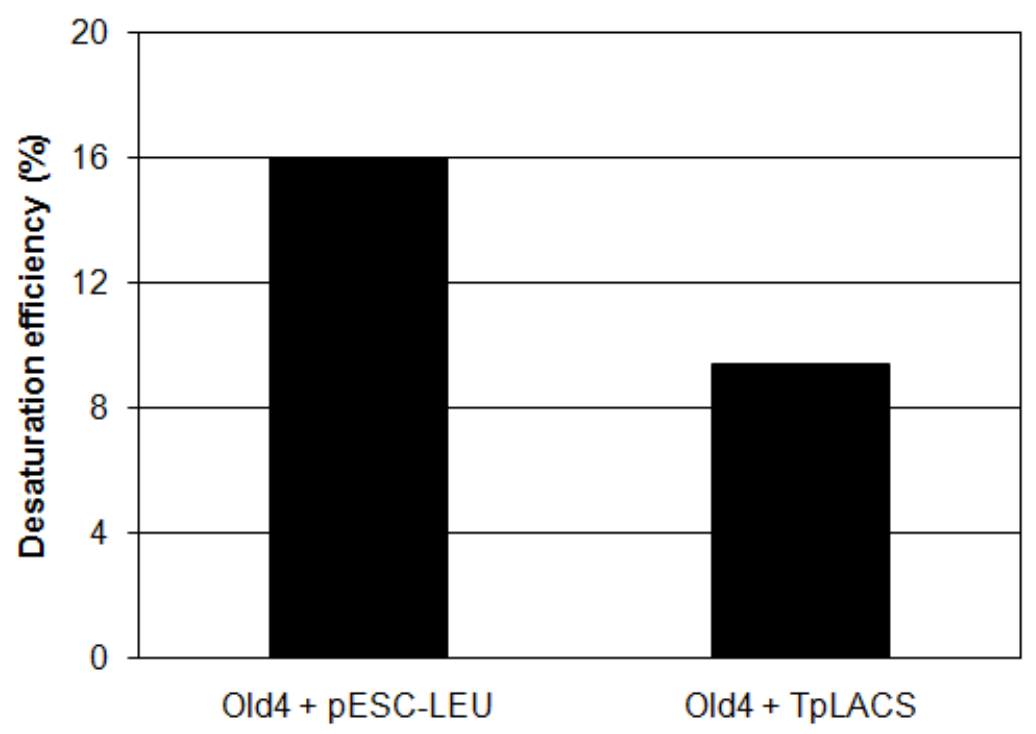

Figure 19. Conversion rates of 22:5(n-3) after co-expression of Old4 with TpLACS in yeast

Old4-pESC-TRP was co-expressed with pESC-LEU or TpLACS-pESC-LEU for three days at $23^{\circ} \mathrm{C}$ in INVSc1 cells supplemented with $200 \mu \mathrm{M}$ 22:5(n-3). FAMEs from yeast cells were prepared and analyzed by GC-FID. Desaturation efficiency was calculated as $100^{*}$ product/(substrate + product). Bars represent the result of one experiment.

Some chloroplastidic desaturases have been shown to act specifically on acyl chains that are linked to galactolipids like MGDG (Norman et al., 1991; Heilmann et al., 2004; Gao et al., 2009). This lipid is the most abundant chloroplastidic lipid and highly enriched in 16:3(n-3) as well as in 18:3(n-3) (Vijayan and Browse, 2002). Because yeast does not produce galactolipids endogenously, missing substrate in this form might be a reason for low desaturation rates of Old $4 p$ as well. To test this hypothesis, Old $4 p$ was introduced together with the MGDG synthase from C. sativus (CsMGDGS; (Shimojima et al., 1997)) into yeast. CsMGDGS has been successfully used in co-expression studies with chloroplastidic desaturases from $A$. thaliana in yeast before and led to a switch in the double bond positioning of the tested desaturases (Heilmann et al., 2004). Also in these experiments, MGDG was produced in yeast cultures expressing CsMGDGS, as can be seen in Figure 20 A. However, supplemented 22:5(n-3) was not incorporated into galactolipids (data not shown), most probably due to substrate restrictions of the cucumber enzyme. When 16:3(n-3) was supplemented in cultures expressing CsMGDGS and Old4, the fatty acid was subsequently found in the galactolipid fraction. In these cultures, nevertheless, no desaturation product was detectable. In cultures coexpressing Old4 with pESC-LEU, desaturation rates with $1.4 \%$ were comparable to those that were observed for expression of Old4 alone (Figure $20 \mathrm{~B}$ and Figure 17).

In conclusion, Old4p desaturated substrates in a lipid-dependent manner and did not reveal a preference for MGDG-bound acyl chains. 

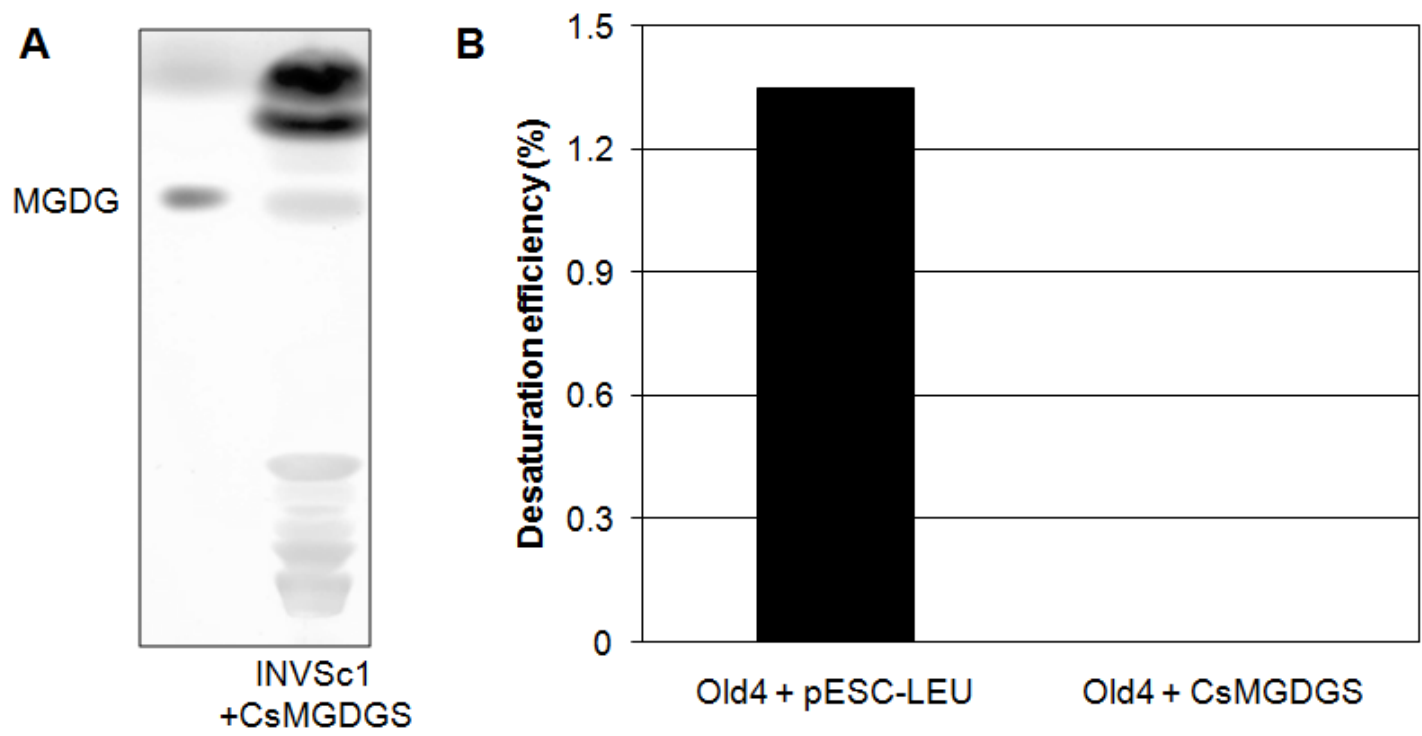

Figure 20. Results after expression of MGDGS and Old4 in yeast

(A) TLC plate after expression of CsMGDGS for three days at $23{ }^{\circ} \mathrm{C}$ in INVSc1, subsequent lipid extraction and separation. (B) Conversion rates of 16:3(n-3) after co-expression of Old4 with CsMGDGS. Old4-pESC-TRP was co-expressed with pESC-LEU or CsMGDGS-pESC-LEU for three days at $23^{\circ} \mathrm{C}$ in INVSc1 cells supplemented with $200 \mu \mathrm{M}$ 16:3(n-3). FAMEs from yeast cells were prepared and analyzed by GC-FID. Desaturation efficiency was calculated as 100* product/(substrate + product). MGDG, monogalactosyldiacylglycerol. Bars represent the mean of two independent experiments.

\subsubsection{Comparative expression of Old4 and Egd4 and localization tests}

To find out whether the rather weak desaturation activity of Old4p was due to low protein levels in yeast, Western Blot studies were performed to compare protein amounts accumulating after expression of Old4 and Egd4, respectively. For these studies, both desaturase cDNAs were cloned into the pUG36 vector (by courtesy of Dr. R. Krick) in order to obtain aminoterminal GFP-tagged fusion proteins. pUG36 contains a negatively regulated methionine-dependent promoter that drives expression in absence of methionine. Yeast cultures expressing the constructs were grown for two days at $23^{\circ} \mathrm{C}$ and under different methionine concentrations in order to test different expression levels. Afterwards, cultures were harvested and protein lysates were produced. These were then analyzed by Western Blot under usage of a GFP-specific antibody. Figure 21 shows similar levels of both desaturase fusion proteins after moderate expression (+ $0.3 \mathrm{mM}$ Met) as well as after over-expression (- Met). To check whether mislocalization of the microalgal desaturase Old4p causes the low enzyme activity, yeast cells harbouring the GFP-fusion construct were examined under the microscope. Old4p did not form aggregates in yeast cells and seemed to localize to the ER, as can be seen in Figure 21. This reflected the expected localization of a typical microsomal front-end desaturase.

Taken together, these results suggested that neither low protein amounts nor enzyme mislocalization were diminishing factors for desaturase activity of Old4p. 
A

\begin{tabular}{|c|c|c|c|c|c|c|}
\hline \multirow[b]{2}{*}{ kDa } & \multicolumn{3}{|c|}{$+0.3 \mathrm{mM}$ Met } & \multicolumn{3}{|c|}{ - Met } \\
\hline & Old4p & Egd4p & - & Old4p & Egd4p & - \\
\hline 86 & -4 & + & & $\longrightarrow$ & $\longrightarrow$ & \\
\hline
\end{tabular}

B

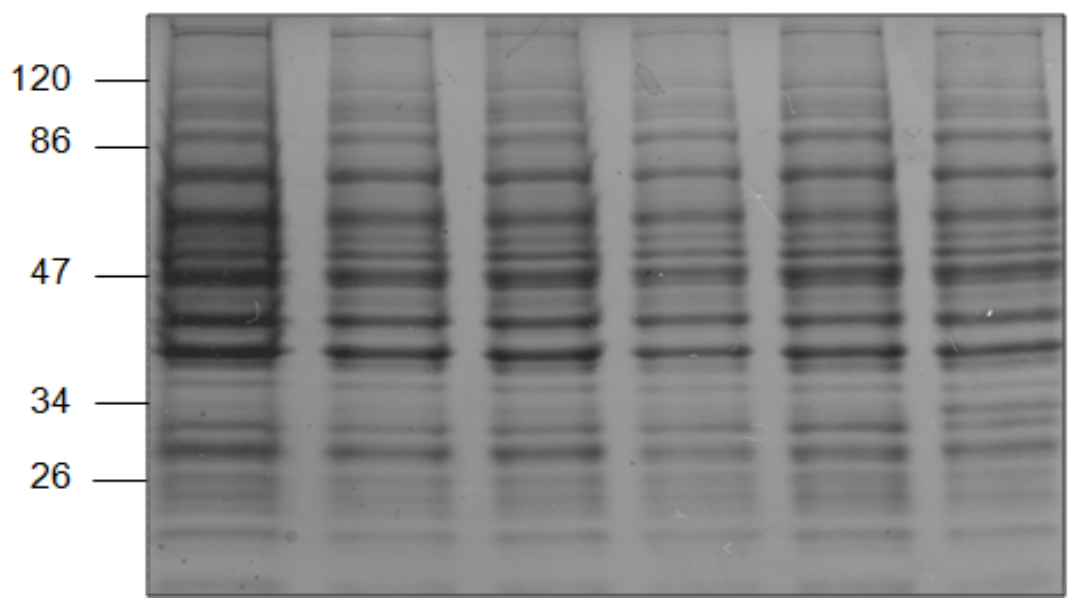

Figure 21. Protein amounts after moderate and strong expression of GFP-Old4

\section{and GFP-Egd4}

GFP-Old4-pUG36, GFP-Egd4-pUG36 or pUG36 (-; negative control) were expressed for two days at $23^{\circ} \mathrm{C}$ in INVSc1 either in presence of $0.3 \mathrm{mM}$ methionine (+ $0.3 \mathrm{mM}$ Met; moderate expression) or in complete absence of methionine (- Met; strong expression). Cells were lysed and total lysates were analyzed. (A) Western Blot and (B) corresponding Coomassie gel as loading control. GFP-Old4p is $78 \mathrm{kDa}$, GFP-Egd4p is $87 \mathrm{kDa}$ in size. The experiment was repeated two times with similar results.

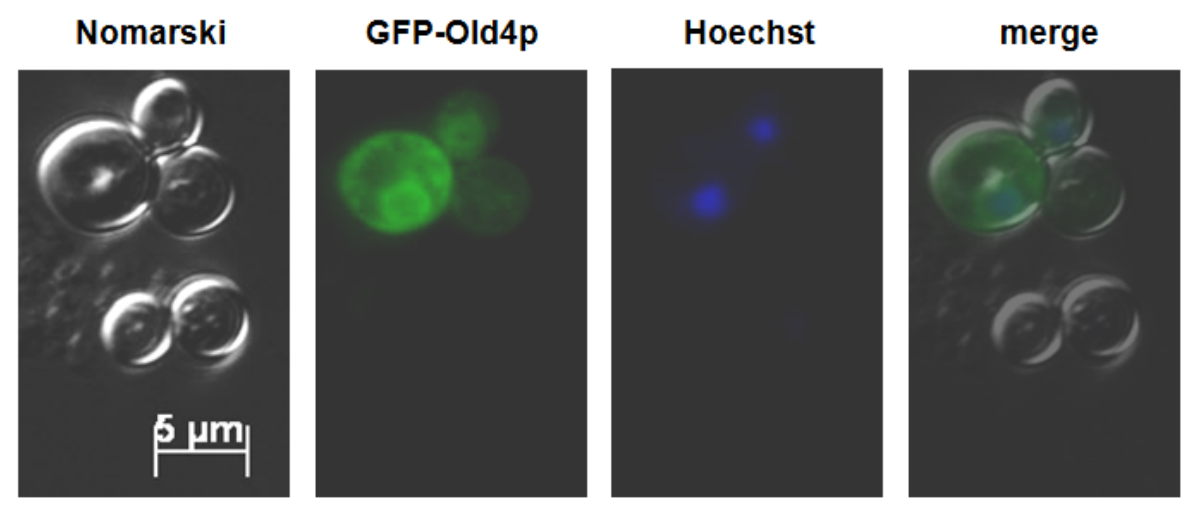

Figure 22. Localization of GFP-Old4p in yeast cells

INVSc1 cells expressing GFP-Old4-pUG36 were grown to stationary phase at $30^{\circ} \mathrm{C}$. Cell nuclei were stained and cells were examined under the microscope. Presented is a representative result of two independent experiments. 


\subsection{Characterization of acyltransferases, desaturases and elongases in plants}

After establishing VLCPUFA production in plants, this process was investigated thoroughly and was extended by further enzymatic activities. This implicated the analysis of microalgal acyltransferases in planta as well as the new combination of already identified and characterized desaturases, elongases and acyltransferases, the transformation of the corresponding sequences into different plant species and subsequent analysis of transgenic seeds, sometimes also throughout consecutive generations.

\subsubsection{Expression of microalgal acyltransferase sequences in $A$. thaliana}

The previously analyzed microalgal acyltransferase sequences were expressed in $A$. thaliana in order to find out which effect they have on the seed lipid metabolism. Therefore, the partly or completely codon-optimized sequences of OtDGAT2B, OILPCAT, OtLPCAT and OtPDAT were cloned behind the seed-specific LeB4 promoter into pCAMBIA33.2cGs and subsequently transformed into $A$. thaliana plants. Alongside with the mentioned constructs, the empty vector was transformed as negative control into Arabidopsis. T1 seeds were harvested and Basta-selected. The derived T2 seeds then were analyzed with respect to their TAG content as well as to their fatty acid composition. Results from TAG content determinations are depicted in Figure 23. No significant changes could be observed upon expression of different acyltransferase sequences in seeds in comparison to the seeds expressing empty vector control.

Results from the determination of fatty acid composition of transgenic Arabidopsis seeds expressing different acyltransferase sequences are depicted in Figure 24 and Figure 25. Expression of OtDGAT2B did not change the fatty acid composition, as can be seen in Figure $24 \mathrm{~A}$. Expression of OtPDAT led to minor changes, enhancing $20: 1(n-9), 20: 2$ and $22: 1$ about $2.2 \%, 0.3 \%$ and $0.6 \%$, respectively. At the same time, levels of $18: 0$ and $18: 1(n-7)$ were decreasing about $0.3 \%$ and $0.7 \%$ in comparison to those seeds expressing the empty vector control (Figure $24 \mathrm{~B}$ ). In seeds expressing OILPCAT, the strongest effects were observed (Figure $25 \mathrm{~A}$ ). 18:0 and 18:1(n-9) amounts were decreasing about $0.3 \%$ or $3.7 \%$, respectively. Concomitantly, 18:2(n-6) and $18: 3(n-3)$ levels were increasing about $1.1 \%$ or $2.8 \%$ in comparison to the control. Further changes were observable for levels of 16:0 (increase of 0.8\%), 16:1 (increase of $0.11 \%$ ), 20:1(n-9) (decrease of $1.5 \%$ ) and 20:2 (increase of $0.4 \%$ ). Expression of OtLPCAT also led to a small increase in $18: 3(n-3)$ of $1 \%$. At the same time, $20: 1(n-7)$ amounts were $0.4 \%$ larger and 20:0 amounts were decreasing about $0.2 \%$ (Figure $25 \mathrm{~B}$ ). 

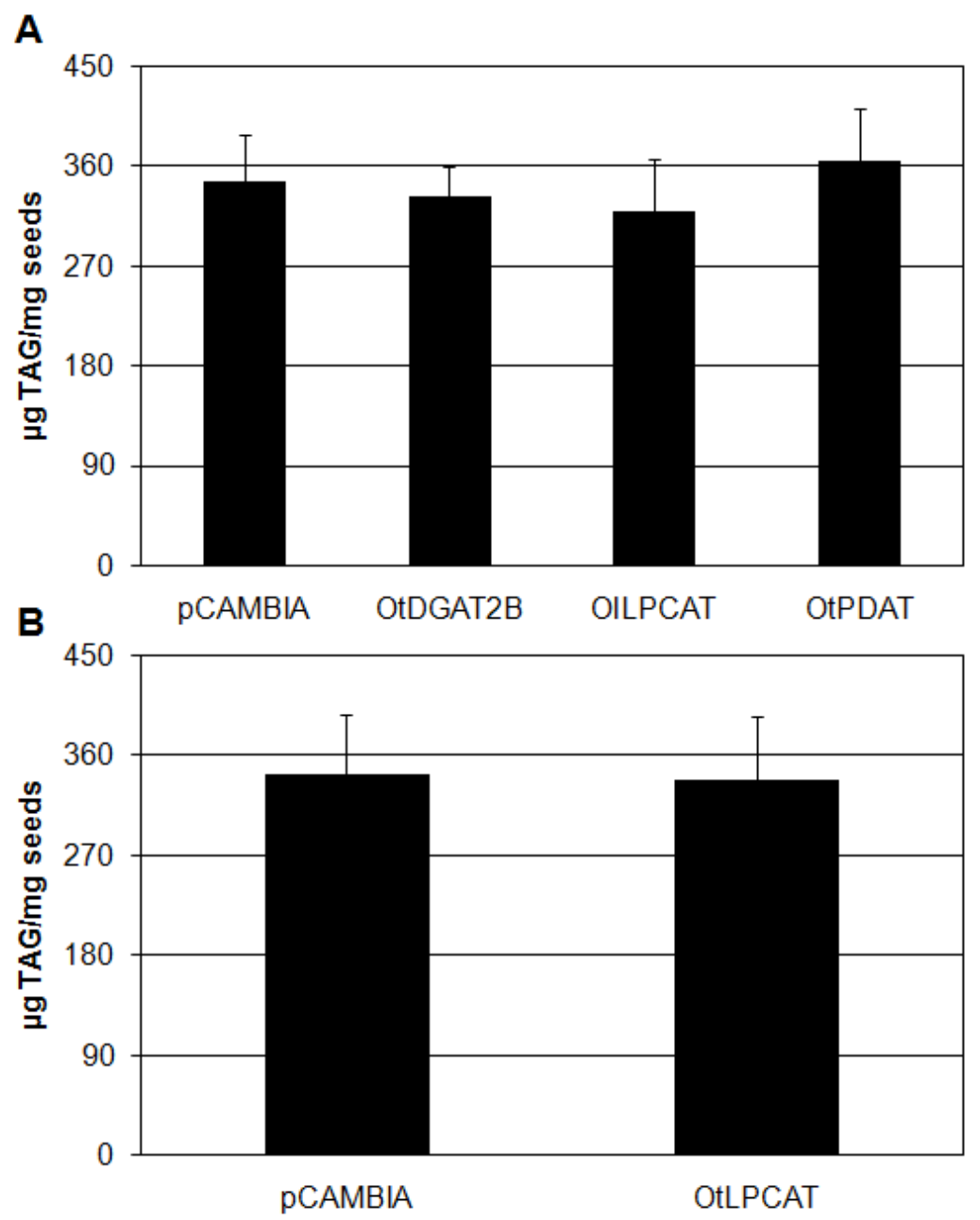

Figure 23. TAG content after expression of microalgal acyltransferase sequences in Arabidopsis

(A) TAG content of T2 seeds expressing pCAMBIA33.2cGs (negative control), OtDGAT2BpCAMBIA33.2cGs, OILPCAT-pCAMBIA33.2cGs or OtPDAT-pCAMBIA33.2cGs. (B) TAG content of seeds expressing pCAMBIA33.2cGs (negative control) or OtLPCAT-pCAMBIA33.2cGs after pool analysis of $3 \mathrm{mg}$ seeds per plant line. TAG, triacylglycerol. Bars represent mean values $+S D$ of ten to twenty independent plant lines. 
A

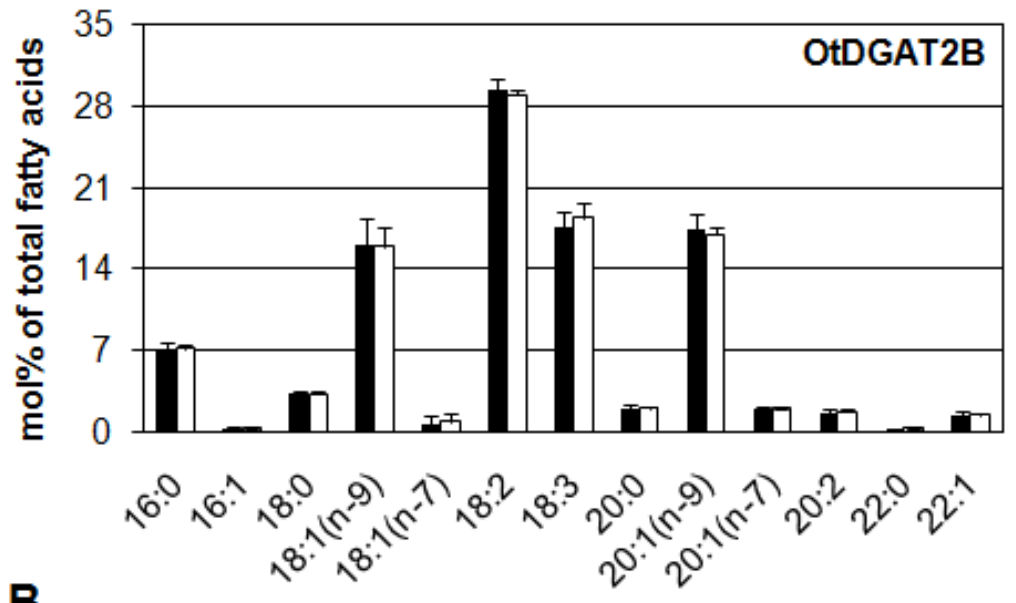

B

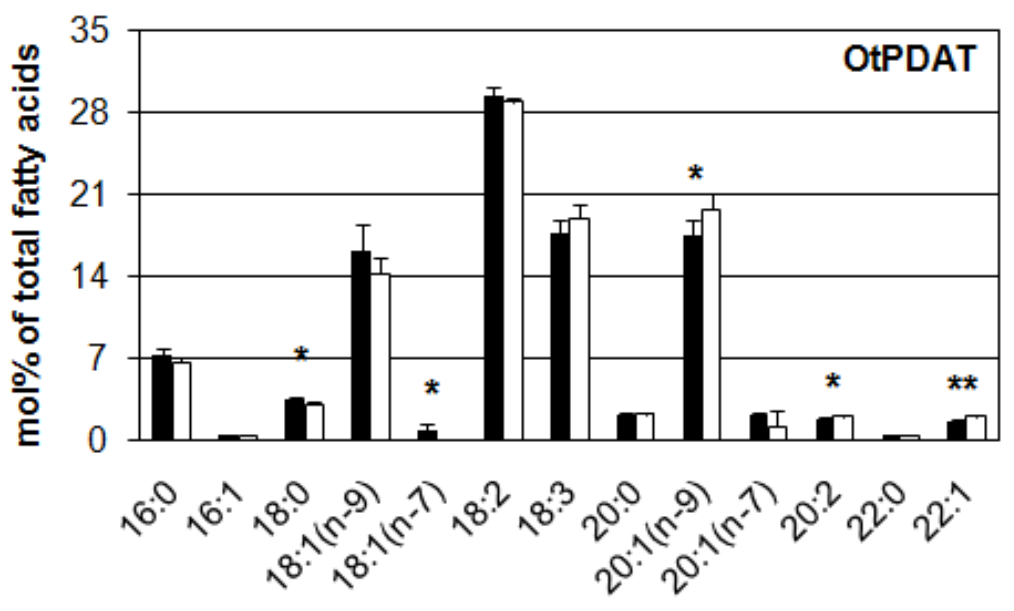

$\square$ pCAMBIA $\square$ acyltransferase

Figure 24. Fatty acid composition after expression of OtDGAT2B and OtPDAT in Arabidopsis seeds

Fatty acid composition of total lipids after pool analysis of $3 \mathrm{mg}$ T2 seeds expressing pCAMBIA33.2cGs (negative control) compared to (A) OtDGAT2B-pCAMBIA33.2cGs or (B) OtPDATpCAMBIA33.2cGs. Bars represent mean values + SD of ten independent plant lines. ${ }^{*}, p<0.01$ and ${ }^{* *}, p<0.001$ determined with Student's $t$-test. 


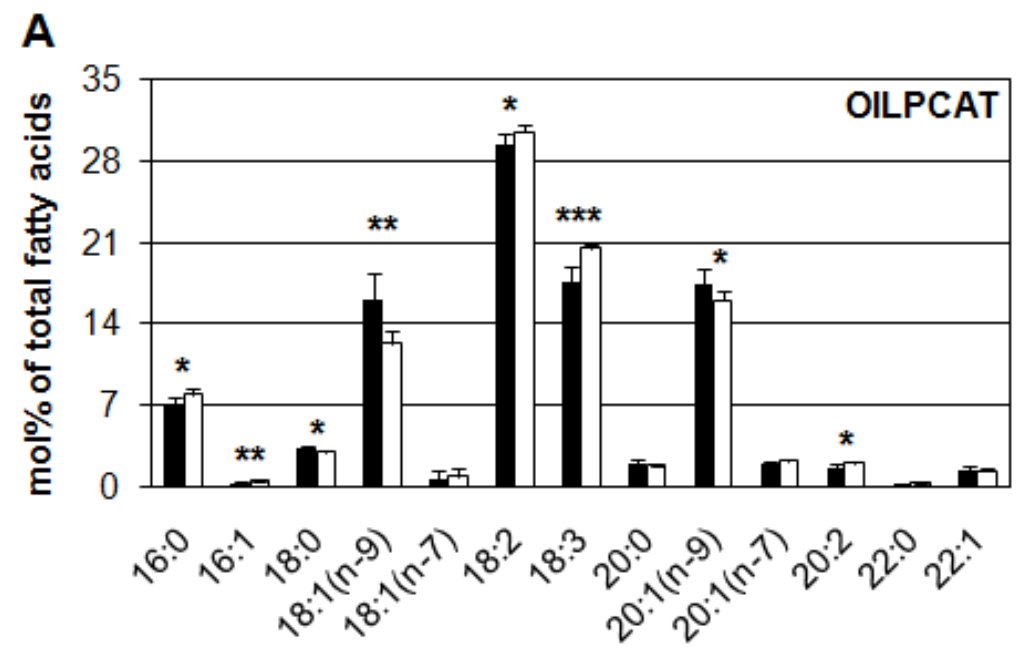

B

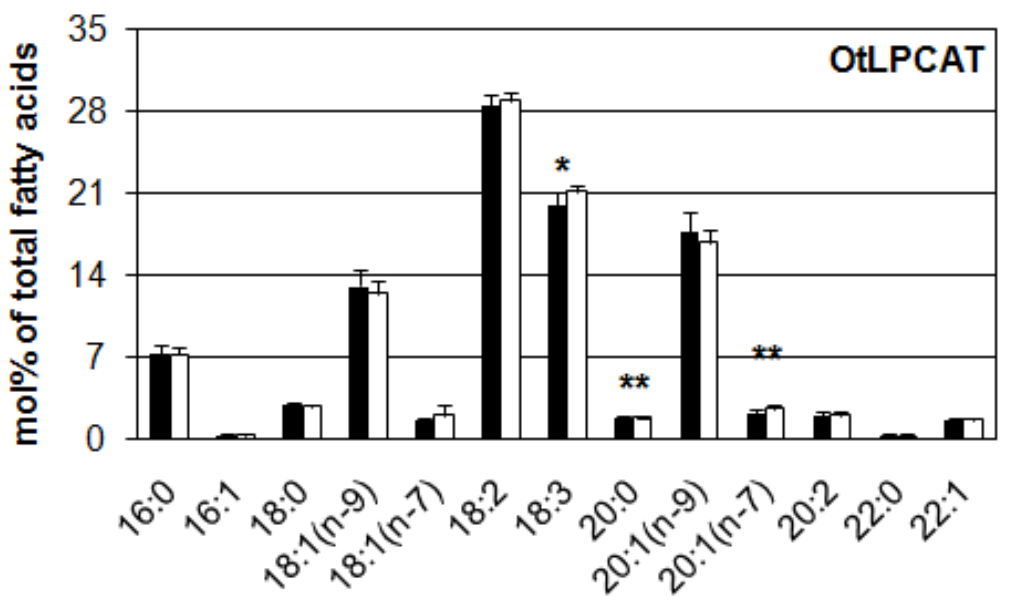

pCAMBIA $\square$ acyltransferase

Figure 25. Fatty acid composition after expression of OILPCAT and OtLPCAT in Arabidopsis seeds

Fatty acid composition of total lipids after pool analysis of $3 \mathrm{mg}$ T2 seeds expressing pCAMBIA33.2cGs (negative control) compared to (A) OILPCAT-pCAMBIA33.2cGs or (B) OtLPCATpCAMBIA33.2cGs. Bars represent mean values + SD of ten to twenty independent plant lines. ${ }^{*}, p<0.01$ and ${ }^{* *}, p<0.001$ and ${ }^{* * *}, p<0.0001$ determined with Student's $t$-test.

Because OILPCAT expression seemed to increase 18:3(n-3) amounts at the expense of 18:1(n-9) in total lipids, it was tested which lipid class is affected in transgenic seeds. For this purpose, total lipids were extracted and the most prominent lipid fractions in seeds, the TAG and the PC fraction, were analyzed. Fatty acid composition of both fractions was compared with those from control plants expressing empty vector. Results from these analyses are depicted in Figure 26. No differences in the fatty acid composition of PC between control seeds and OILPCAT-expressing seeds could be observed (Figure $26 \mathrm{~A}$ ). In the TAG fraction, however, an increase in 18:2(n-6) amounts of $1.3 \%$ and in $18: 3(n-3)$ amounts of $2 \%$ was detectable. At the same time, 
18:0 und 20:0 levels were decreasing about $0.5 \%$ and $0.4 \%$, respectively (Figure 26 B). Because seeds expressing empty vector showed a high standard deviation in 18:1(n-9)-levels, no significant difference could be observed in comparison to seeds expressing OILPCAT.

In summary, expression of OILPCAT showed the strongest effects in Arabidopsis seeds by decreasing 18:1(n-9) levels and increasing amounts of polyunsaturated 18carbon chain fatty acids. These changes could be pinpointed to the TAG fraction of seeds.

A

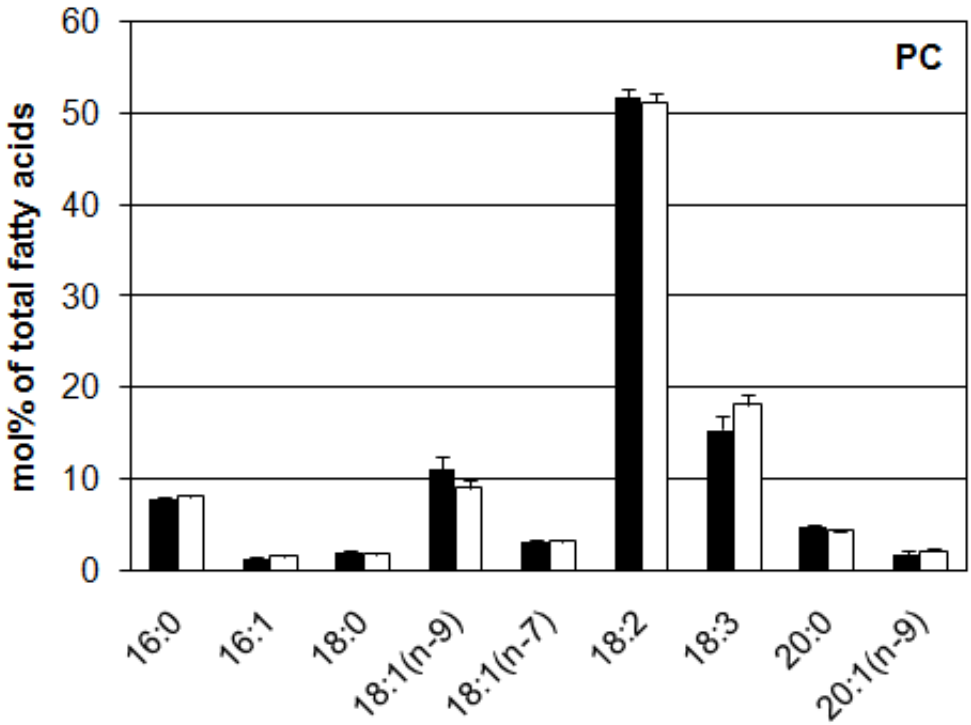

- $\mathrm{pCAMBIA}$

口OILPCAT

B

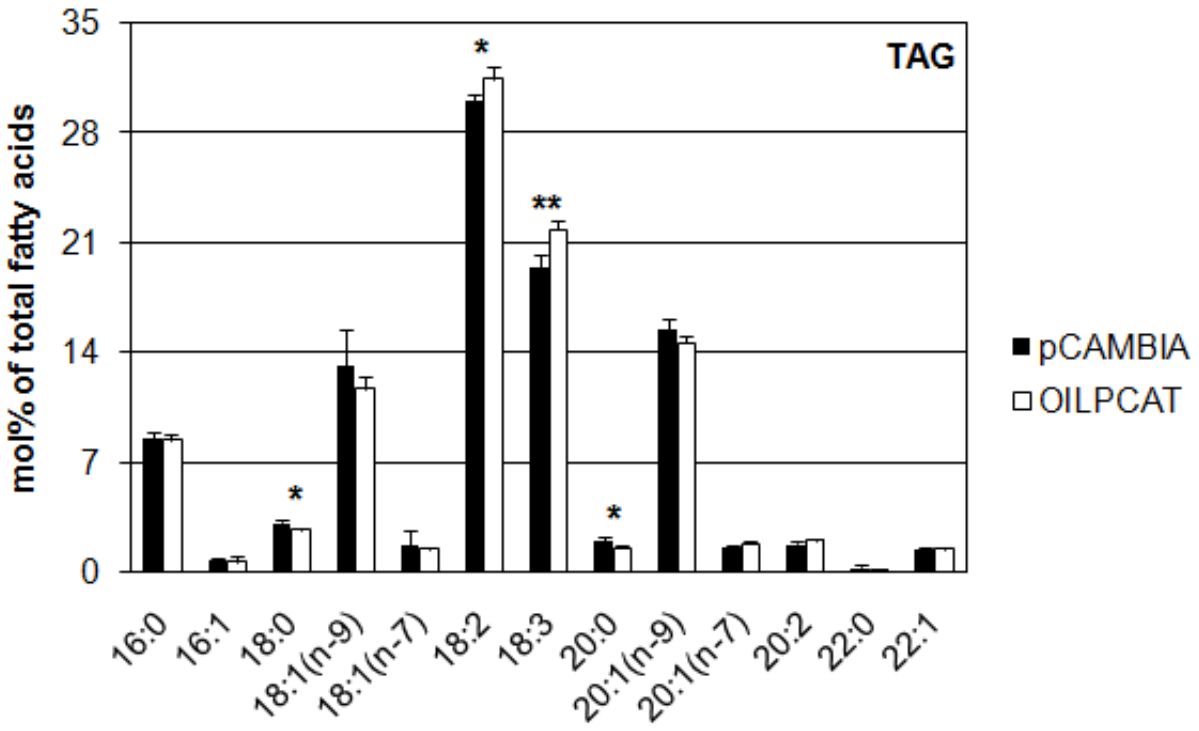

Figure 26. PC and TAG fatty acid compositions after expression of OILPCAT in Arabidopsis

Fatty acid composition of (A) PC and (B) TAG from $3 \mathrm{mg}$ T2 seeds expressing pCAMBIA33.2cGs (negative control) compared to OILPCAT-pCAMBIA33.2cGs was determined by total lipid extraction, separation and GC analysis of different lipid fractions. PC, phosphatidylcholine; TAG, triacylglycerol. Bars represent mean values + SD of five independent plant lines. ${ }^{*}, p<0.01 ;{ }^{* *}, p<0.001$ determined with Student's $t$-test. 


\subsubsection{Complementation experiments in the $A$. thaliana tag1-1 mutant}

The Arabidopsis tag1-1 mutant, also known as AS11 mutant, has been identified in an ethane methyl sulfonate screen by Katavic and co-workers in 1995. It revealed much higer levels of ALA (18:3(n-3)) and diminished levels of oleic acid (18:1(n-9)) as well as eicosenoic acid (20:1(n-9)) in mature seeds (Katavic et al., 1995). In further studies, mutant seeds were shown to also have a strongly reduced TAG content in comparison to wild type seeds (Zou et al., 1999). The mutated gene was identified to encode a DGAT1p and was supposed to be a major factor influencing TAG content and composition in Arabidopsis seeds (Katavic et al., 1995; Routaboul et al., 1999; Zou et al., 1999).

Mutant seeds were analyzed side-by-side with wild type seeds in order to check whether effects that are described in literature could be reproduced under our experimental conditions. For this purpose, mutant and wild type plants were cultivated in the climate chamber and their total seed lipids were analyzed with respect to their TAG content and their fatty acid composition. Results of these measurements are depicted in Figure 27. Overall TAG content in mutant seeds was with $250 \mu \mathrm{g} / \mathrm{mg}$ seeds about $100 \mu \mathrm{g}$ lower than TAG content of wild type seeds, as can be seen in Figure $27 \mathrm{~A}$. Fatty acid composition of mutant seeds was also altered in comparison to wild type seeds with higher levels of 18:3(n-3) and lower levels of 18:1 and 20:1 (Figure 27 B). Taken together, these results confirmed the data obtained by other groups.

Next, it was tested whether expression of the microalgal OtDGAT2B is able to rescue the observed phenotype. Thus, the construct OtDGAT2B-pCAMBIA33.2cGs carrying the acyltransferase sequence downstream of the seed-specific LeB4-promoter was transformed into tag1-1 mutant plants. As positive controls, AtDGAT1 and AtDGAT2 from Arabidopsis were brought into the same expression vector and were also transformed into the mutant. As negative control, empty vector was transformed into tag 1-1 plants. As further control, wild type Arabidopsis plants transformed with empty vector were used. T1 plants were cultivated in the greenhouse, Basta-selected and their T2 seeds were used for analyses of TAG content and fatty acid composition of total seed lipids. Results from TAG analyses are illustrated in Figure 28. Surprisingly, TAG amounts were not significantly different in transgenic wild type and mutant lines containing empty vector or expressing different DGAT sequences. Overall, TAG amounts were with about $250 \mu \mathrm{g}$ TAG/mg seeds as low as in the tag1-1 mutant seeds analyzed before (Figure 27 and Figure 28). 

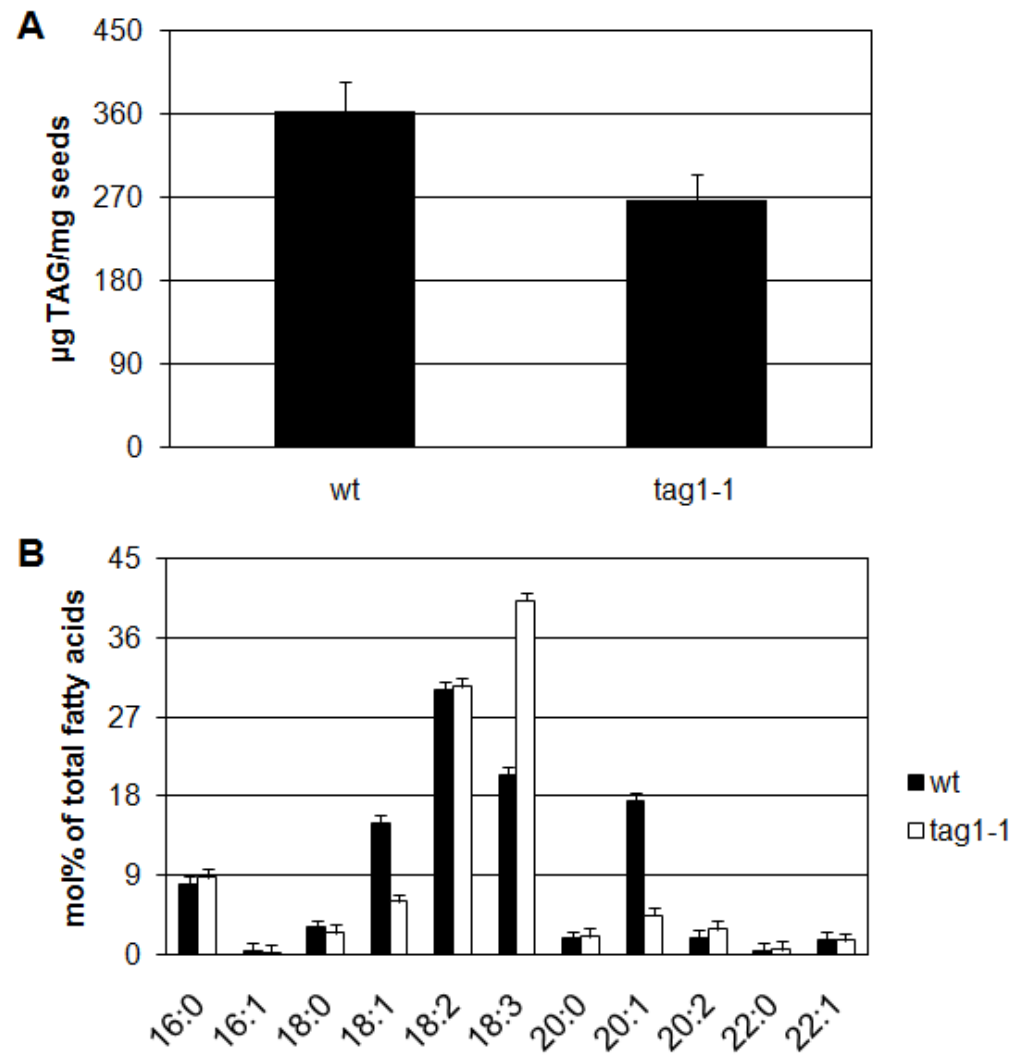

Figure 27. TAG content and fatty acid composition of Arabidopsis wt and tag1-1 seeds

(A) TAG content and (B) fatty acid profile of Col-0 (wt) and tag1-1 mutant seeds from pool analysis of $3 \mathrm{mg}$ seeds per plant. TAG, triacylglycerol. Bars represent mean $+\mathrm{SD}$ of ten different plants.

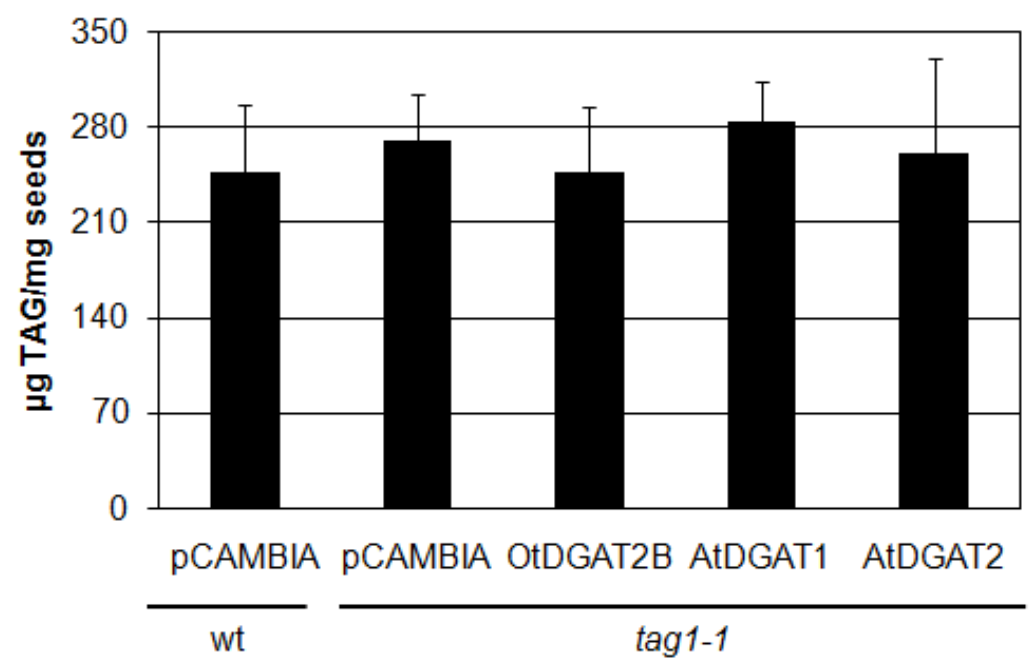

Figure 28. TAG content after expression of different DGAT sequences in Arabidopsis wild type and tag 1-1 mutant seeds

TAG content of $3 \mathrm{mg}$ Col-0 (wt) or mutant T2 seeds expressing pCAMBIA33.2cGs, OtDGAT2BpCAMBIA33.2cGs, AtDGAT1-pCAMBIA33.2cGs or AtDGAT2-pCAMBIA33.2cGs from pool analysis. TAG, triacylglycerol. Bars represent mean values + SD of ten different plants. 
Results from the analyses concerning fatty acid composition of total seed lipids are depicted in Figure 29. In these measurements, the previously observed strong effects of the DGAT1-mutation could be confirmed. 18:3(n-3) levels were increased in tag1-1 mutant seeds, whereas 18:1(n-9) and 20:1(n-9) levels were reduced in comparison to Col-0 wild type seeds. However, no significant differences between expression of empty vector, OtDGAT2B, AtDGAT1 or AtDGAT2 could be observed. Thus, it was not possible to rescue the mutant phenotype with the utilized DGAT constructs.

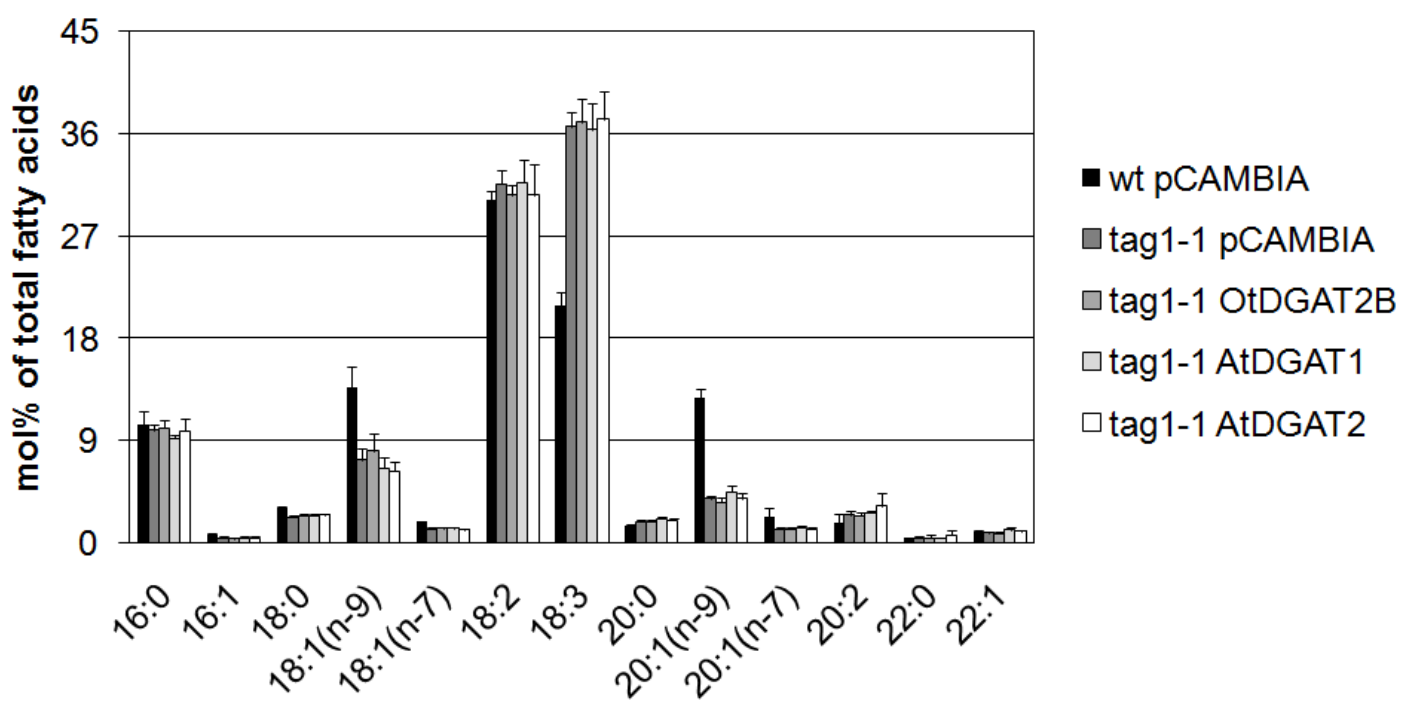

Figure 29. Fatty acid composition after expression of different DGAT sequences in Arabidopsis wild type and tag1-1 mutant seeds

Fatty acid composition of total lipids of $3 \mathrm{mg}$ wild type or mutant T2 seeds expressing pCAMBIA33.2cGs, OtDGAT2B-pCAMBIA33.2cGs, AtDGAT1-pCAMBIA33.2cGs or AtDGAT2pCAMBIA33.2cGs. Bars represent mean values + SD of ten independent plant lines.

\subsubsection{Combination of different desaturases with an elongase in $A$. thaliana}

During previous studies, Dr. M. Heilmann isolated acyl-CoA-dependent desaturases from $O$. tauri $(\Delta 5$-desaturase $\operatorname{Otd} 5 p)$ and $M$. squamata $(\Delta 5$ - and $\Delta 6$-desaturases Msd5p and Msd6p, respectively). Their corresponding cDNA sequences were coexpressed under the control of the unspecific seed protein (USP) promoter with the previously identified $\Delta 6$-desaturase sequence from 0 . tauri (Otd6, (Domergue et al., 2005)) and the moss elongase sequence PSE1 (Zank et al., 2002) in the constructs Otd6-PSE1-Otd5-pCAMBIA3300 (Ot3) and Msd6-PSE1-Msd5-pCAMBIA3300 (Ms3) in A. thaliana to produce EPA in transgenic seeds. As control, the construct Ptd6-PSE1Ptd5-pCAMBIA3300 (Pt3) containing, besides PSE1, the lipid-dependent $P$. tricornutum $\Delta 6$ - and $\Delta 5$-desaturase sequences Ptd6 and Ptd5 described by (Abbadi et al., 2004) under control of the USP promoter, was also transformed into Arabidopsis. EPA amounts after expression of Ms3 and Pt3 were found to be about $0.03 \%$, EPA 
amounts after expression of Ot3 were about $0.02 \%$ of total fatty acids in the analyzed T2 seeds (Hoffmann et al., 2008). It was tested how the amounts of newly formed fatty acids alter in the consecutive seed generation. Therefore, transgenic T2 seeds from 22-30 Basta-selected T1 plants, representing independent transformation events for each expression construct, were analyzed. Two to four plant lines for each expression construct containing the highest amounts of (n-3)-PUFAs (sum from percentages of 18:4(n-3), 20:4(n-3) and 20:5(n-3) in total fatty acids) in their T2 seeds were then chosen for analysis in the T3 generation. Results of the screen are shown in Table 3. The percentage of (n-3)-PUFAs in T2 seeds expressing Ms3 was on average at $1.72 \%$ showing a standard deviation of $1.27 \%$. Analysis of T3 seeds from different Ms3 T2 plant lines led to inconsistent results. Half of the examined lines showed higher amounts of (n-3)-PUFAs of $2.46 \%$ and $2.51 \%$, respectively, in the next generation. The other T2 plant lines contained lower mean percentages of (n-3)-PUFAs of $1.12 \%$ and $0.73 \%$ in their T3 seeds. Also, the determined standard deviations were not declining. A similar trend could be observed also in case of (n-3)-PUFAs after Pt3 and Ot3 expression. The only exception in this respect constituted the (n-6)-PUFA percentages after expression of $\mathrm{Pt} 3$ which were on average at $3.53 \%$ for T2 seeds, but at 5.15 $\%$ and $7.27 \%$ for T3 seeds. Furthermore, determined standard deviations were quite high in these analyses. In conclusion, no uniform development of PUFA amounts in the course of different generations could be observed. 
Table 3. Overview about (n-3)- and (n-6)-PUFA amounts in Arabidopsis seeds after expression of different VLCPUFA constructs

Pool analysis for $3 \mathrm{mg}$ seeds per plant was performed. Ms3, Msd6-PSE1-Msd5-pCAMBIA3300; Pt3, Ptd6-PSE1-Ptd5-pCAMBIA3300; Ot3, Otd6-PSE1-Otd5-pCAMBIA3300. N indicates the number of tested plants. (n-3)-PUFA, sum from percentages of 18:4(n-3), 20:4(n-3) and $20: 5(n-3)$ in total fatty acids; (n-6)-PUFA, sum from percentages of 18:3(n-6), 20:3(n-6) and $20: 4(n-6)$ in total fatty acids. Mean values \pm SD for percentages of newly formed fatty acids from total fatty acids are given.

\begin{tabular}{|ccccc|}
\hline construct & seed generation & $\mathbf{N}$ & (n-3)-PUFA (\%) & (n-6)-PUFA (\%) \\
\hline Ms3 & T2 & 22 & $1.72 \pm 1.27$ & $0.51 \pm 0.60$ \\
\hline Ot3 & T2 & 28 & $1.94 \pm 0.59$ & $1.05 \pm 0.67$ \\
\hline Pt3 & T2 & 30 & $1.88 \pm 1.07$ & $3.53 \pm 4.05$ \\
\hline Ms3 & T3 & 30 & $2.46 \pm 2.42$ & $0.62 \pm 0.74$ \\
\hline Ms3 & T3 & 29 & $2.51 \pm 1.68$ & $0.26 \pm 0.38$ \\
\hline Ms3 & T3 & 28 & $1.12 \pm 0.50$ & $0.01 \pm 0.05$ \\
\hline Ms3 & T3 & 29 & $0.73 \pm 0.33$ & $0.02 \pm 0.10$ \\
\hline Ot3 & T3 & 29 & $1.18 \pm 0.43$ & $0.80 \pm 0.21$ \\
\hline Ot3 & T3 & 31 & $2.16 \pm 0.40$ & $1.56 \pm 0.46$ \\
\hline Ot3 & T3 & 30 & $1.63 \pm 1.07$ & $1.15 \pm 0.95$ \\
\hline Pt3 & T3 & 29 & $1.29 \pm 0.28$ & $5.15 \pm 1.09$ \\
\hline Pt3 & T3 & 30 & $2.09 \pm 1.15$ & $7.27 \pm 5.70$ \\
\hline
\end{tabular}




\subsubsection{Combination of acyl-CoA- and lipid-dependent desaturases in A. thaliana}

The combination of lipid-dependent desaturases with an acyl-CoA-dependent elongase has been shown to lead to the accumulation of intermediates after the first desaturation step in linseed and tobacco. A possible reason for this is the insufficient transfer of substrates between PC pool and acyl-CoA pool (Abbadi et al., 2004). In subsequent studies, the utilization of acyl-CoA-dependent desaturases was shown to enhance substrate flux through the metabolic pathway of VLCPUFA production in plants. The only remaining problem was the low activity of the identified acyl-CoA-dependent desaturases with $\Delta 5$-specificity (Hoffmann et al., 2008). The following experiment was performed to investigate, whether the combination of the acyl-CoA-dependent desaturase Msd6 $p$ with the lipid-dependent desaturases Ptd6p and Ptd5p together with elongase PSE1p leads to higher EPA levels in seeds compared to the previously tested combinations. Thus, Msd6 was cloned under the control of the seed-specific USP promoter into Ptd6-PSE1-Ptd5-pCAMBIA3300 (Pt3) and the resulting construct was transformed into Arabidopsis. T2 seeds of 13 Basta-selected independent T1 plant lines were screened for presence of newly produced fatty acids of the VLCPUFA pathways. Unfortunately, none of the tested lines revealed VLCPUFAs (data not shown). In a second approach, also the OILPCATp from $O$. lucimarinus was combined with the lipiddependent desaturase and PSE1p under the control of the USP promoter to check whether the putative acyltransferase is able to transfer intermediates of the pathway between the different acyl pools in seeds and thereby circumvents the described bottleneck. OILPCAT hence was cloned into $\mathrm{Pt} 3$ and the resulting construct was transformed into $A$. thaliana. T2 seeds of 30 independent T1 plant lines were screened, but identified VLCPUFA amounts were much lower compared to Pt3 plants (data not shown). In a third approach, Pt3 was combined with both Msd6 and OILPCAT in order to find out whether co-expression leads to higher VLCPUFA yields in transgenic Arabidopsis seeds. Thus, both sequences were cloned into Pt3 and the resulting construct was transformed into plants. Results of the co-expression compared to beforehand detected (n-3)-PUFA levels (Hoffmann et al., 2008) are depicted in Figure 30. The first intermediate of the pathway, 18:4(n-3), was accumulating with $2 \%$ at much higher levels in seeds expressing OILPCAT-Msd6-Pt3 compared to seeds expressing Pt3 $(0.7 \%)$ or Ms3 (0.1\%). 20:4(n-3) amounts were with $0.9-1 \%$ comparable in seeds expressing OILPCAT-Msd6-Pt3 or Ms3. However, EPA yields after expression of OILPCAT-Msd6-Pt3 with $0.3 \%$ were much higher than after expression of Pt3 or Ms3, that both led to EPA amounts of $0.05 \%$. Generally, one should keep in mind that plants were cultivated at different times and product levels might be influenced by several other factors apart from the utilized enzymatic activities. 
In conclusion, co-expression of OILPCAT with Msd6, Ptd6, PSE1 and Ptd5 led to much higher EPA levels in Arabidopsis seeds compared to the constructs tested in previous studies.

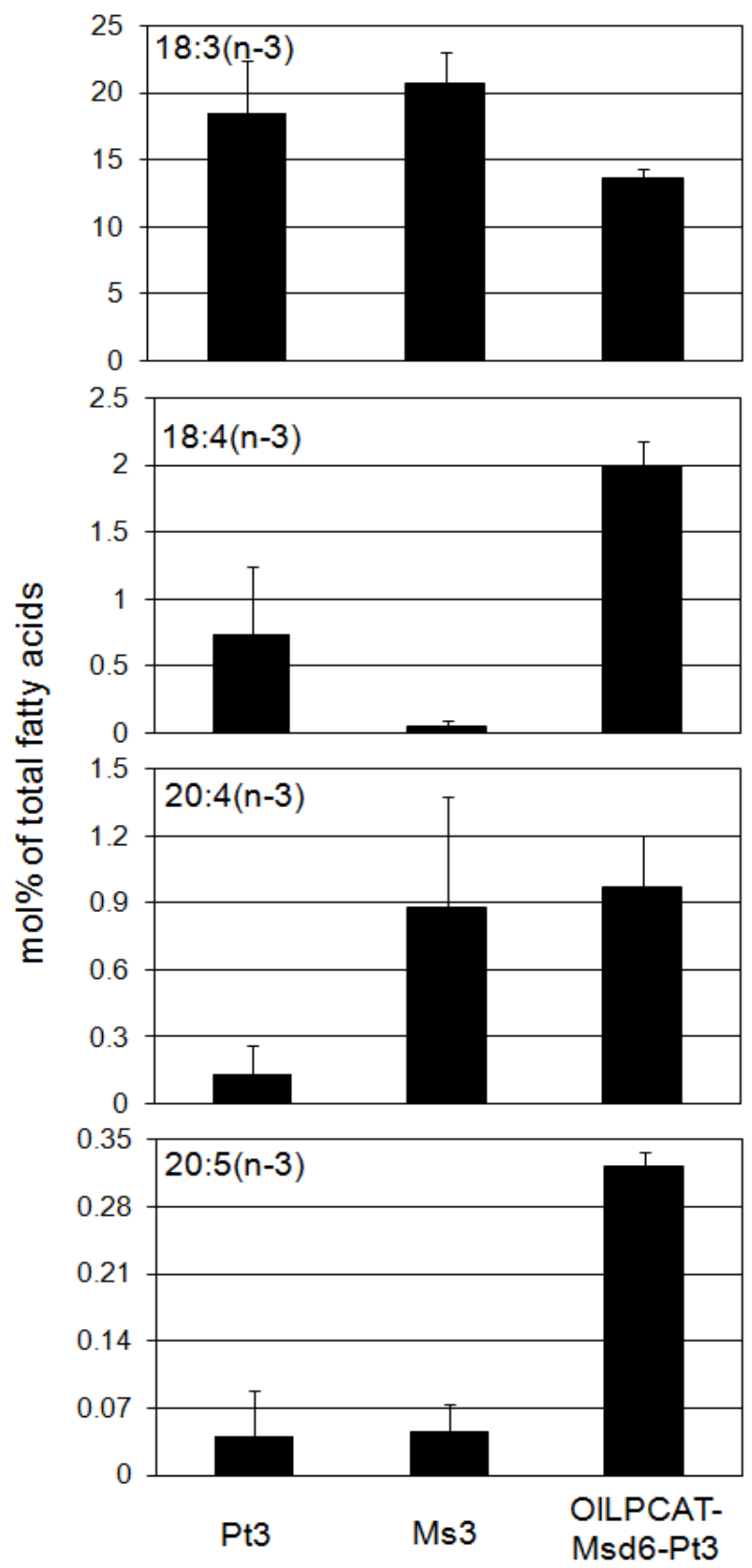

Figure 30. (n-3)-PUFA amounts in Arabidopsis seeds after expression of different

\section{VLCPUFA constructs}

Pt3, Ptd6-PSE1-Ptd5-pCAMBIA3300; Ms3, Msd6-PSE1-Msd5-pCAMBIA3300. Pool analysis of $3 \mathrm{mg}$ seeds per plant line was performed. Values from Pt3 and Ms3 plants are taken from (Hoffmann et al., 2008). Bars represent mean + SD of three independent plant lines. 


\subsubsection{Comparison of different host plants for VLCPUFA biosynthesis}

For VLCPUFA production, various plant species like for example tobacco, linseed, soy, oilseed rape, Arabidopsis, Indian or Ethiopian mustard have already been utilized as host organisms (Abbadi et al., 2004; Qi et al., 2004; Robert et al., 2005; Wu et al., 2005; Hoffmann et al., 2008; Cheng et al., 2010). From these studies it could be learned that the choice of the host plants can have a great influence on product yields (Cheng et al., 2010). So far, our VLCPUFA constructs were tested solely in Arabidopsis. In order to figure out whether product yields can be increased by utilization of another, more suitable host plant, comparative studies between transgenic Camelina sativa and $A$. thaliana plants were performed.

C. sativa is an ancient oilseed crop. It is a member of the Brassiceae family and has been grown extensively in the nineteenth century (Knorzer, 1978). For unknown reasons, cultivation of Camelina diminished after the Second World War. However, in recent times this plant species has come back into focus of research due to its healthpromoting fatty acid composition (Lu and Kang, 2008). In Figure 31, fatty acid profiles of $A$. thaliana and $C$. sativa wild type seeds are displayed. Camelina seeds are very rich in 18:3(n-3), resulting in a high (n-3) over (n-6) ratio. Arabidopsis seeds in contrast have a fatty acid composition that is typical for popular oilseed crops like oilseed rape or soybean. Their most prominent fatty acid is the (n-6)-fatty acid 18:2(n-6). That is why their $(n-3)$ over $(n-6)$ ratio is rather low.

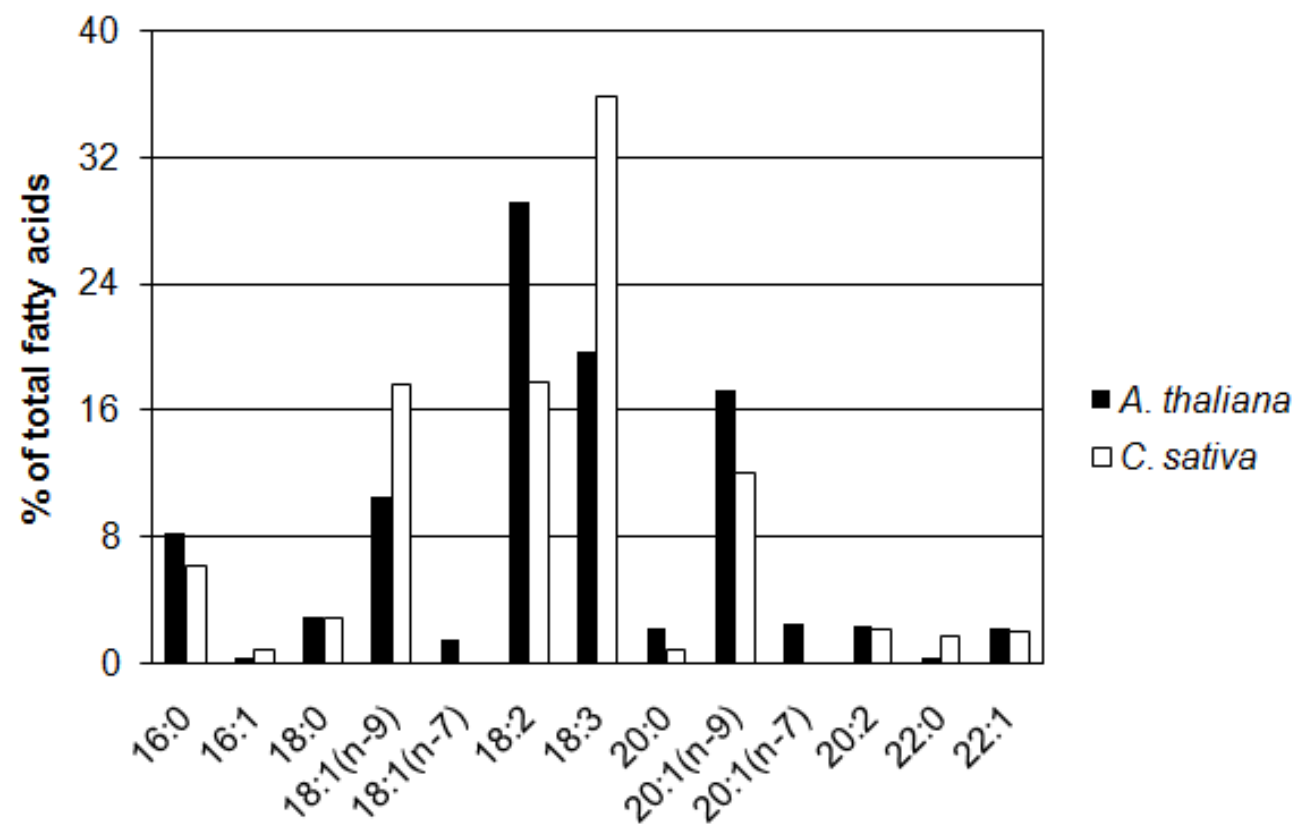

Figure 31. Fatty acid composition of $\boldsymbol{A}$. thaliana and $\boldsymbol{C}$. sativa wild type seeds

For $C$. sativa, single wild type seeds were analyzed, for $A$. thaliana, seed pool analysis of $3 \mathrm{mg}$ seeds per plant was performed. Bars represent mean values from two independent samples. 
Based on these observations, $C$. sativa was regarded as suitable production system for (n-3)-PUFAs because of its high initial substrate amounts. Therefore, flowering Camelina plants were transformed with Ptd6-PSE1-Ptd5-pCAMBIA3300 (Pt3) or the empty vector control based on the method developed by (Lu and Kang, 2008). T1 seeds were harvested and Basta-selected. Work concerning Camelina plants was done in cooperation with V. Behnen. T2 seeds were analyzed subsequently by single seed analysis and results were compared to Pt3-transformed T2 Arabidopsis seeds. T2 seeds were sown again. After a second selection round, T3 seeds were analyzed and also compared to transgenic Arabidopsis T3 seeds. Results of these comparative studies are illustrated in Figure 32 and Figure 33. As can be seen in Figure 32 A and B, Arabidopsis seeds in the T2 generation generally showed higher amounts of newly formed fatty acids of both (n-3)- and (n-6)-pathways compared to Camelina seeds. Solely, 20:4(n-6) amounts with $1.25 \%$ were higher in Camelina seeds of the T2 generation. In contrast, amounts of newly formed (VLC)PUFAs were much lower in the analyzed T3 Arabidopsis seeds and rising vice versa in T3 Camelina seeds (Figure 33 $A$ and $B$ ). Amounts of both ( $n-6)$ - and ( $n-3)$-fatty acids in this generation were higher than in Arabidopsis seeds, reaching 20:4(n-6) yields of $4.2 \%$ and EPA yields of $0.4 \%$ of total fatty acids, respectively, in Camelina. It should be noted that transgenic Camelina T3 seeds looked shriveled, and their 18:3(n-3) amounts were about $18 \%$ lower than in the analyzed wild type and transgenic T2 seeds (Figure $33 \mathrm{~B}$, Figure 31 and Figure 32 B). 18:2(n-6) levels were comparable in all transgenic and wild type Camelina seeds (Figure $32 \mathrm{~A}$, Figure $33 \mathrm{~A}$ and Figure 31). The precursor fatty acid 18:1(n-9) with 9-12\% was present at lower levels in transgenic Camelina seeds compared to wild type seeds (Figure 31).

In summary, expression of Pt3 in the two different host organisms Arabidopsis and Camelina led to different product amounts, also revealing differences between the analyzed consecutive generations. 
A
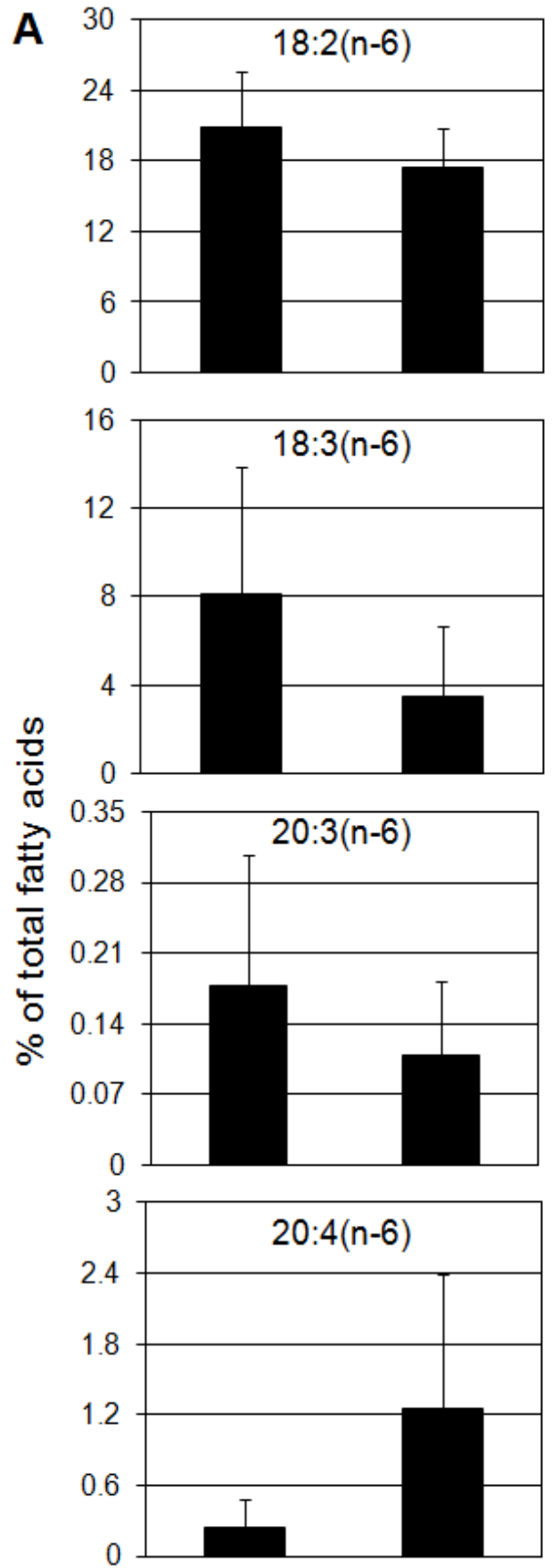

A. thaliana C. sativa
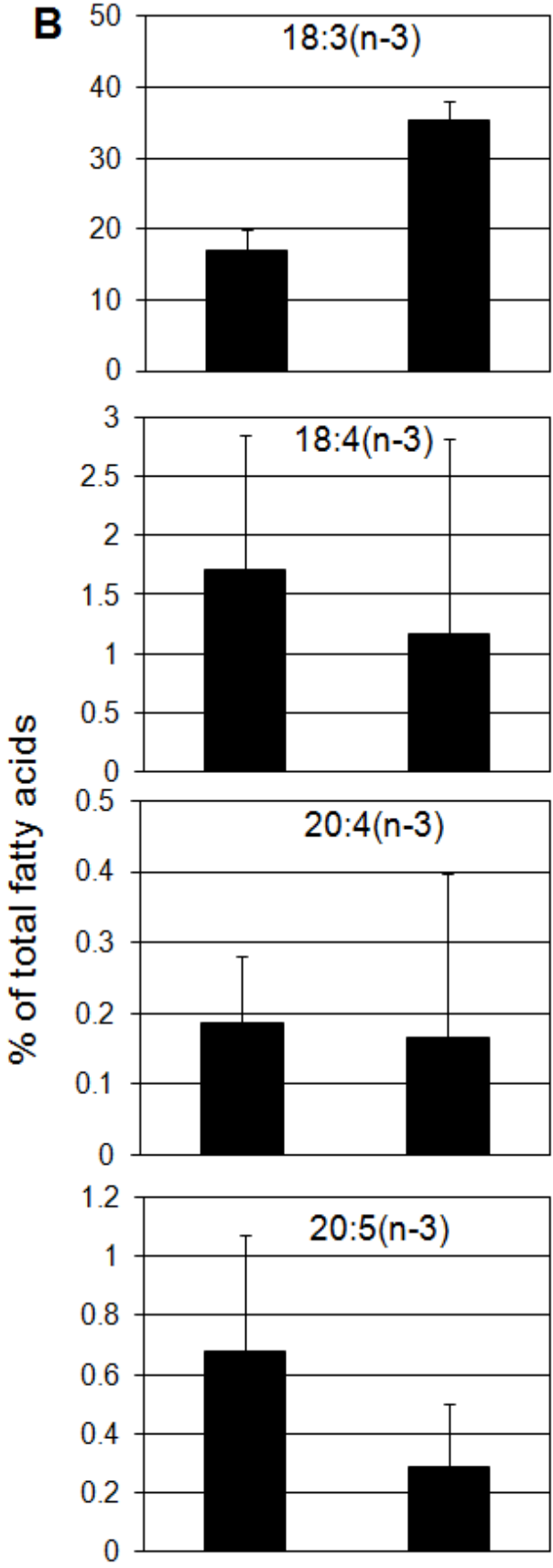

A. thaliana C. sativa

Figure 32. (n-6)- and (n-3)-PUFA contents of transgenic A. thaliana and C. sativa T2 seeds

Plants were transformed with Ptd6-PSE1-Ptd5-pCAMBIA3300, subsequently transgenic $A$. thaliana and $C$. sativa T2 seeds were analyzed by single seed analysis. (A) (n-6)-PUFA values and (B) (n-3)-PUFA values. Bars represent mean + SD from ten independent samples. Transformation of Camelina plants was performed in cooperation with V. Behnen. 

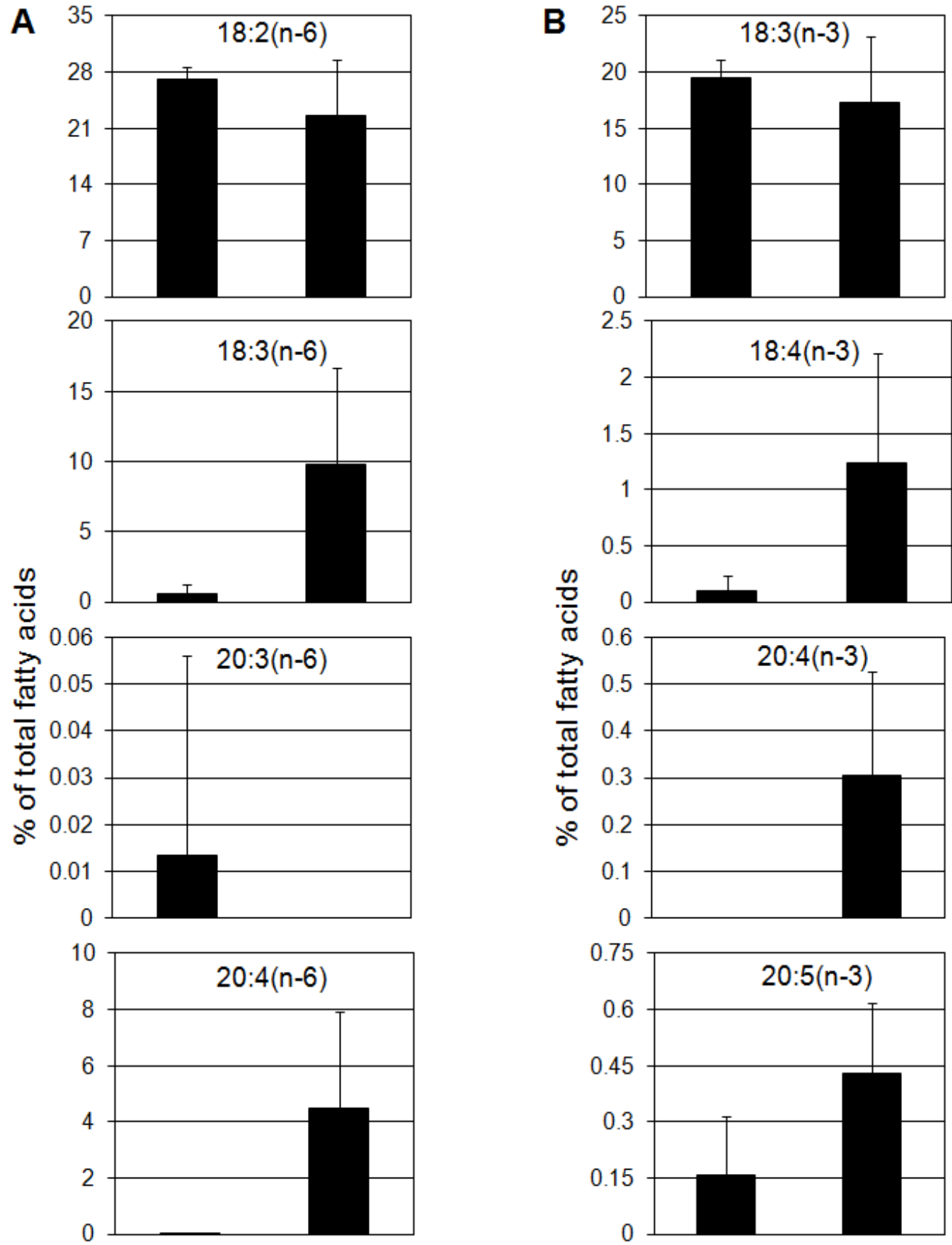

A. thaliana C. sativa

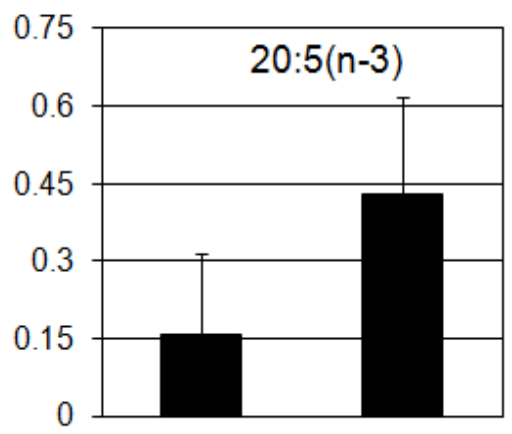

A. thaliana C. sativa

Figure 33. (n-6)- and (n-3)-PUFA contents of transgenic $A$. thaliana and $C$. sativa T3 seeds

Plants were transformed with Ptd6-PSE1-Ptd5-pCAMBIA3300, subsequently transgenic $A$. thaliana and $C$. sativa T3 seeds were analyzed by single seed analysis. (A) (n-6)-PUFA values and (B) (n-3)-PUFA values. Bars represent mean + SD from ten independent samples. Transformation of Camelina plants was performed in cooperation with V. Behnen. 


\subsubsection{Combination of microalgal acyltransferases with desaturases and an elongase in A. thaliana}

After the separated analysis of transgenic seeds expressing acyltransferase sequences or sequences encoding VLCPUFA-producing enzymes, the next step was to investigate the interplay between desaturases, elongases and acyltransferases upon combination in planta. Therefore, Ptd6-PSE1-Ptd5-pCAMBIA2200 (Pt3, (Abbadi et al., 2004)) was transformed in one step alongside with the different acyltransferasepCAMBIA33.2cGs constructs into Arabidopsis plants. T1 seeds were first selected on 1/2 MS medium containing kanamycin to screen for Pt3-positive plants. Subsequently, surviving seedlings were put on soil and were sprayed with Basta to select for acyltransferase-expressing plants. Surviving plants after this second selection were then cultivated and their T2 seeds were used for analysis of TAG content and VLCPUFA yields. Due to two independently transformed constructs and thus two necessary consecutive selection rounds, only few plants survived and were available for analysis. Results from the TAG analyses are shown in Figure 34. No significant differences could be observed in the TAG levels between those seeds co-expressing the empty vector with Pt3 and those expressing OtDGAT2B, OtPDAT, OILPCAT or OtLPCAT together with Pt3. Mean VLCPUFA yields in plant lines co-expressing Pt3 with different acyltransferase sequences are depicted in Figure 35 and Figure 36 . In general, both (n-6)- and (n-3)-VLCPUFA levels were similar in all analyzed plant lines and standard deviations were quite high. Expression of the empty vector together with Pt3 resulted in almost all cases to slightly higher VLCPUFA amounts compared to the co-expression with acyltransferase sequences. In conclusion, co-expression with different acyltransferase sequences did not improve the yield of VLCPUFA production by Pt3 expression. 


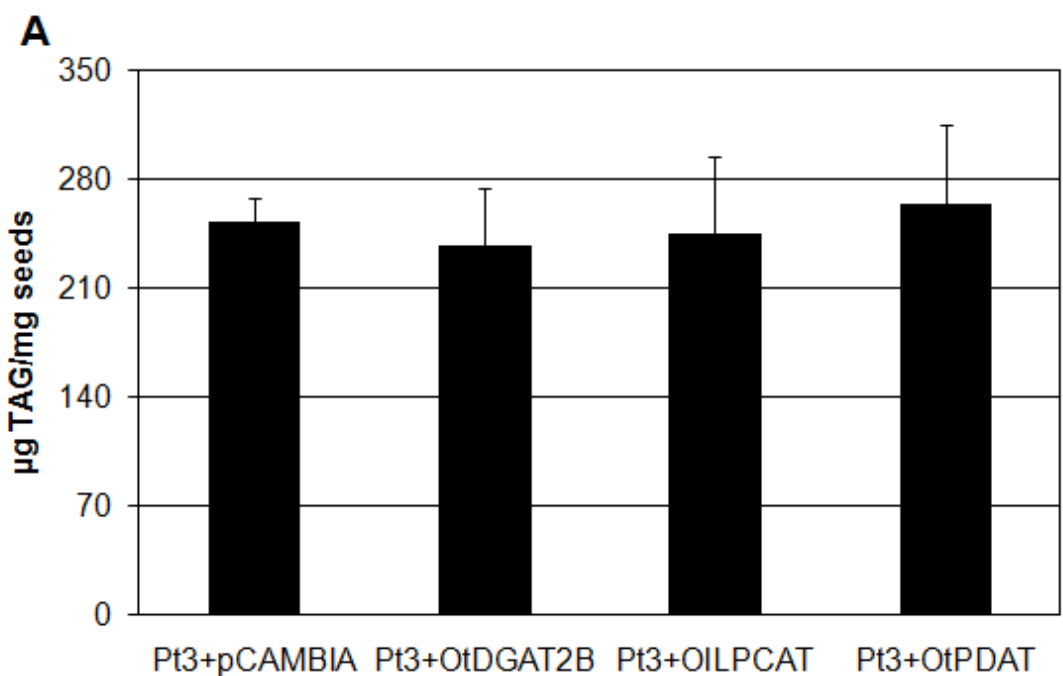

B

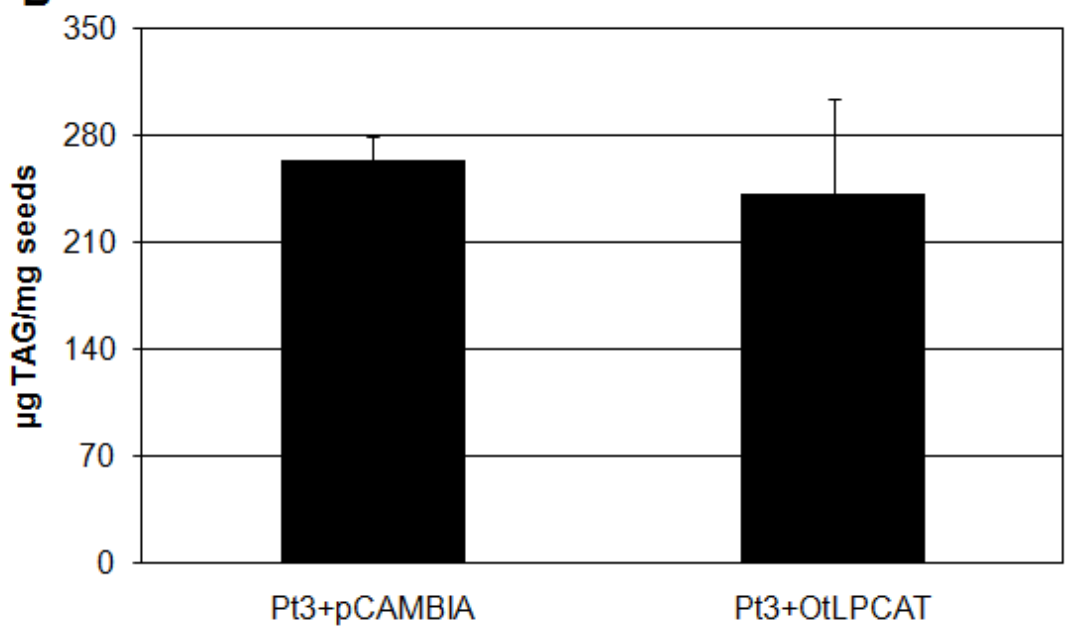

Figure 34. TAG content after co-expression of microalgal acyltransferase sequences with $\mathrm{Pt} 3$ in Arabidopsis

(A) TAG content of $3 \mathrm{mg}$ T2 seeds co-expressing Ptd6-PSE1-Ptd5-pCAMBIA2200 (Pt3) with pCAMBIA33.2cGs, OtDGAT2B-pCAMBIA33.2cGs, OILPCAT-pCAMBIA33.2cGs or OtPDATpCAMBIA33.2cGs. (B) TAG content of $3 \mathrm{mg}$ T2 seeds co-expressing Pt3 with pCAMBIA33.2cGs or OtLPCAT-pCAMBIA33.2cGs. TAG, triacylglycerol. Bars represent mean values $+\mathrm{SD}$ of three to eleven independent plant lines. 
A

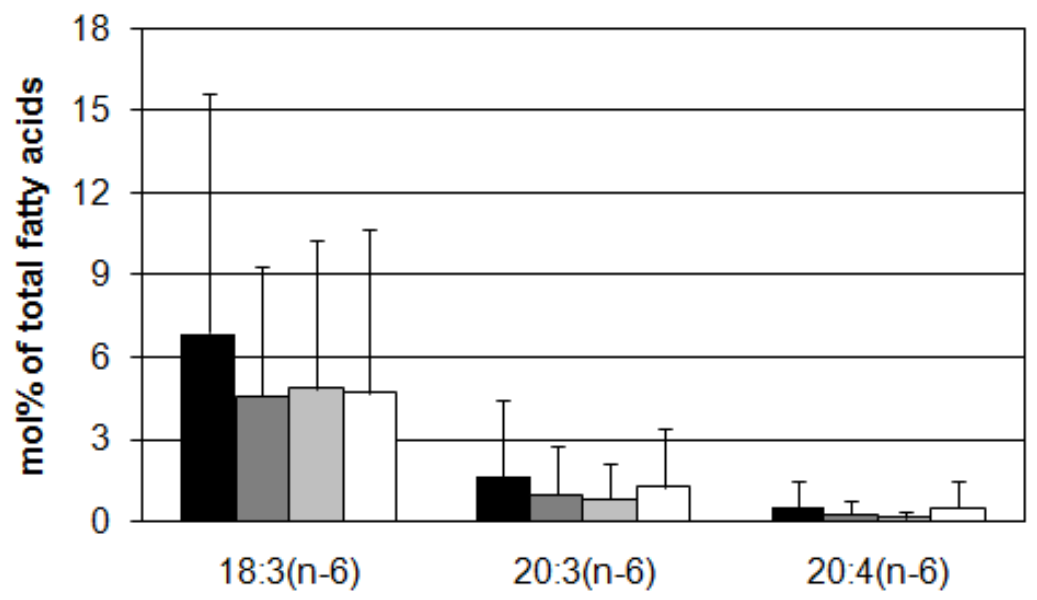

B

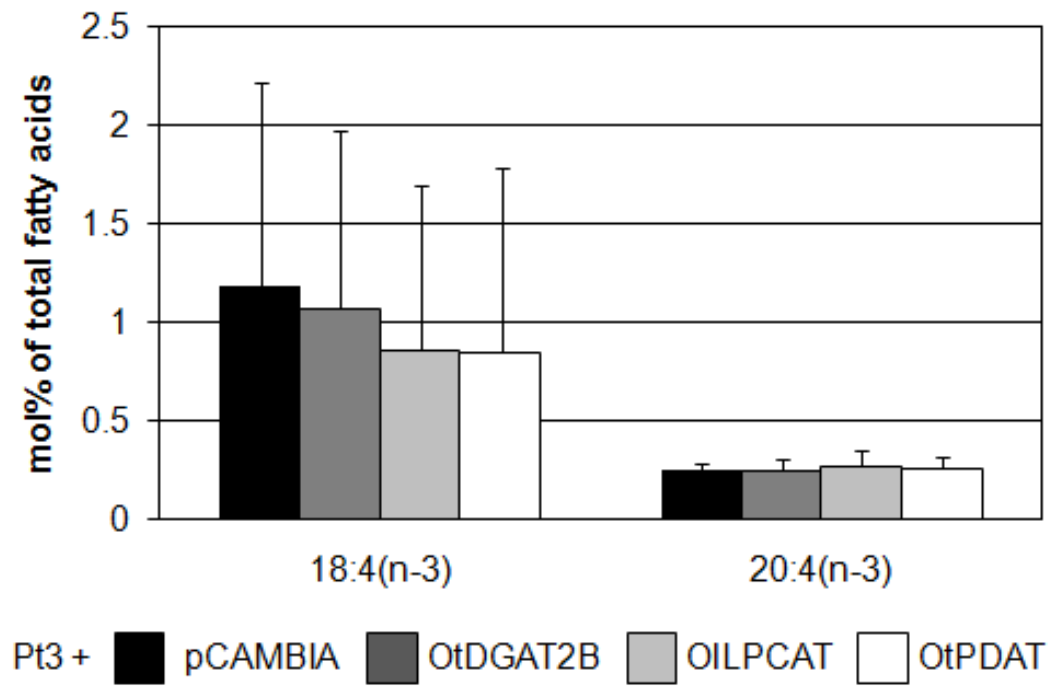

Figure 35. (n-6)- and (n-3)-PUFA contents of transgenic $A$. thaliana seeds coexpressing Pt3 with OtDGAT2B, OILPCAT or OtPDAT

Plants were co-transformed with Ptd6-PSE1-Ptd5-pCAMBIA2200 (Pt3) and pCAMBIA33.2cGs, OtDGAT2B-pCAMBIA33.2cGs, OILPCAT-pCAMBIA33.2cGs or OtPDAT-pCAMBIA33.2cGs. $3 \mathrm{mg}$ T2 seeds were analyzed by pool analysis. (A) (n-6)-PUFA values and (B) (n-3)-PUFA values. Bars represent mean + SD from three to eleven independent plant lines. 
A

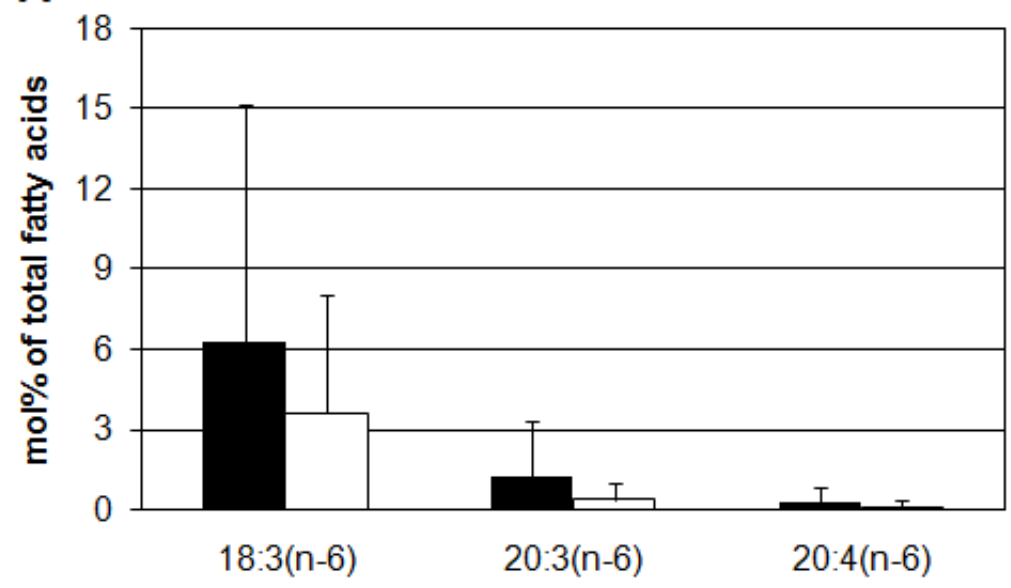

B

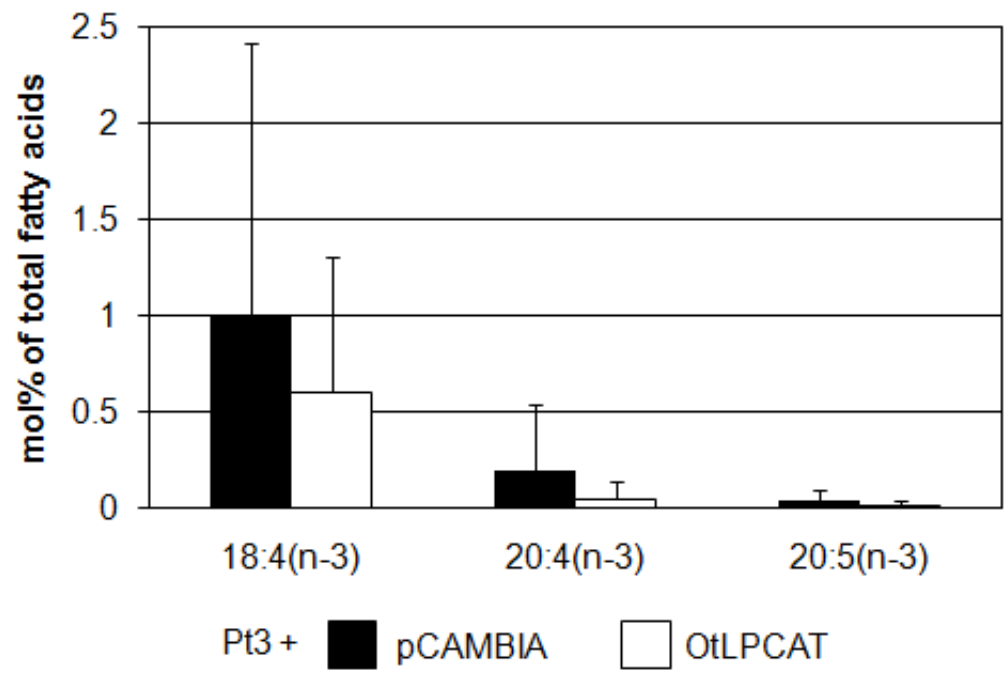

Figure 36. (n-6)- and (n-3)-PUFA contents of transgenic $A$. thaliana seeds coexpressing Pt3 with OtLPCAT

Plants were co-transformed with Ptd6-PSE1-Ptd5-pCAMBIA2200 (Pt3) and pCAMBIA33.2cGs or OtLPCAT-pCAMBIA33.2cGs. $3 \mathrm{mg}$ T2 seeds were analyzed by pool analysis. (A) (n-6)-PUFA values and (B) (n-3)-PUFA values. Bars represent mean + SD from three to eleven independent plant lines. 


\subsubsection{Combination of microalgal acyltransferases with desaturases and an elongase in the $A$. thaliana tag1-1 mutant}

It was also tested whether VLCPUFA levels are higher in the tag1-1 mutant upon expression of Ptd6-PSE1-Ptd5-pCAMBIA2200 (Pt3, (Abbadi et al., 2004)) and which effects co-expression of plant and microalgal DGAT sequences might have. Therefore, Pt3 was co-transformed with the different DGAT-pCAMBIA33.2cGs constructs containing either AtDGAT1 or OtDGAT2B into tag1-1 plants. T1 seeds were selected for presence of both constructs. Surviving plants were then cultivated in the greenhouse and their T2 seeds were used for analysis of TAG content and VLCPUFA yields. Results from the TAG analyses are shown in Figure 37. The overall TAG levels again were with 200-250 $\mathrm{mg}$ TAG/mg seeds as low as those from the non-transformed tag1-1 seeds (Figure 27), but no differences could be observed between the different transgenic lines. Results from the analyses of VLCPUFA yields are illustrated in Figure 38. In the transgenic tag1-1 mutant seeds much lower levels of (n-6)-PUFAs could be detected compared to the beforehand analyzed transgenic wild type seeds (Figure 36). In those mutant seeds expressing empty vector together with $\mathrm{Pt} 3,20: 4(\mathrm{n}-6)$ was not observable at all (Figure $38 \mathrm{~A}$ ). In seeds co-expressing AtDGAT1 or OtDGAT2B with Pt3, at least low 20:4(n-6) levels of about $0.2 \mathrm{~mol} \%$ of total fatty acids were found. Surprisingly, also (n-3)-PUFA values were lower in comparison to wild type seeds, although (n-3)substrate levels should be higher in the mutant seeds. For seeds co-expressing empty vector with Pt3, only 18:4(n-3) was detectable, but no 20:4(n-3) or EPA. However, it should be noted that the number of analyzed independent plant lines was rather low due to the double selection process and standard deviations were quite high.

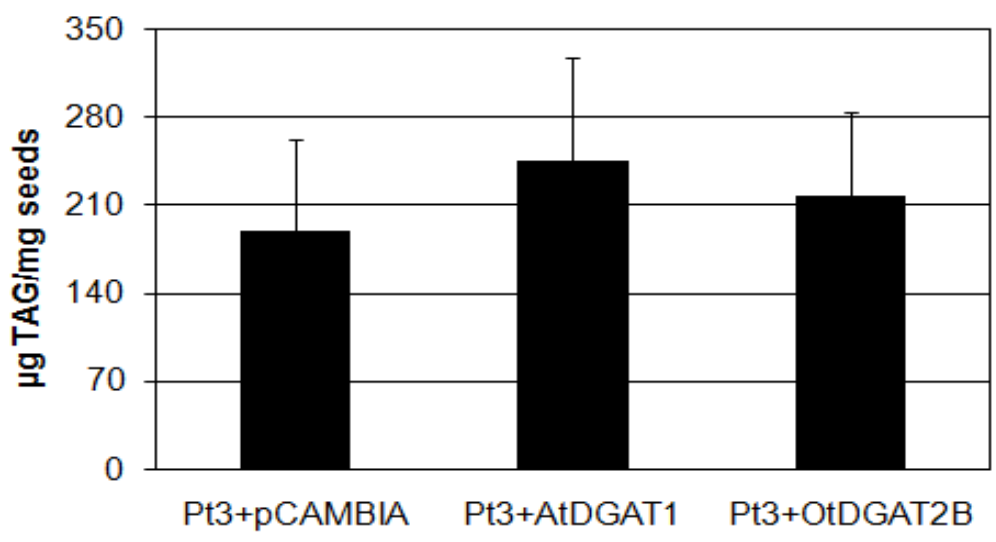

Figure 37. TAG content after co-expression of different acyltransferase sequences with Pt3 in Arabidopsis tag1-1 seeds

TAG content from pool analysis of $3 \mathrm{mg}$ T2 seeds co-expressing Ptd6-PSE1-Ptd5pCAMBIA2200 (Pt3) with pCAMBIA33.2cGs, AtDGAT1-pCAMBIA33.2cGs or OtDGAT2BpCAMBIA33.2cGs. TAG, triacylglycerol. Bars represent mean values + SD of four to six independent plant lines. 
A

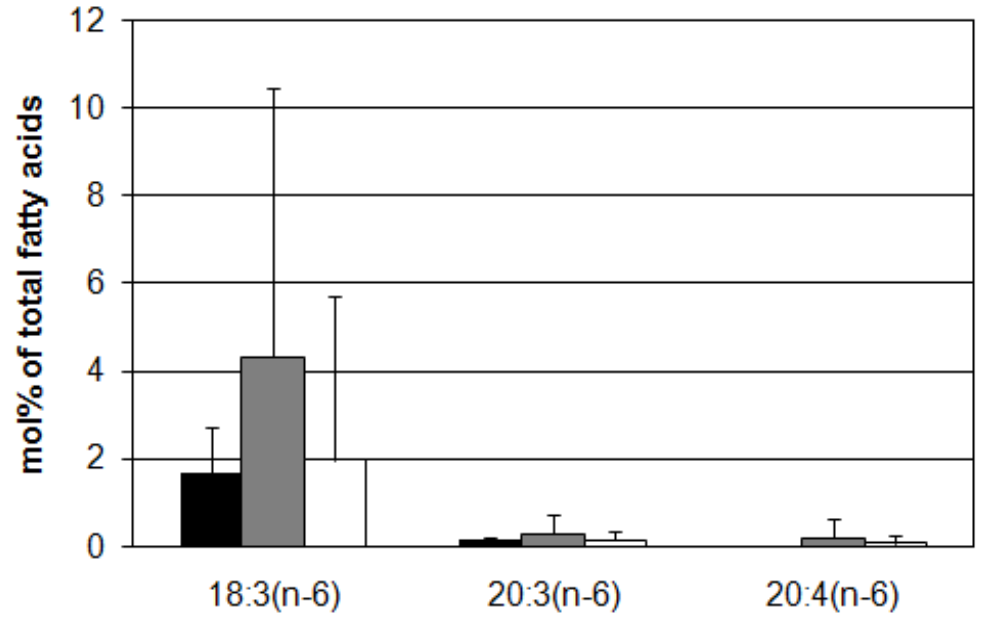

B

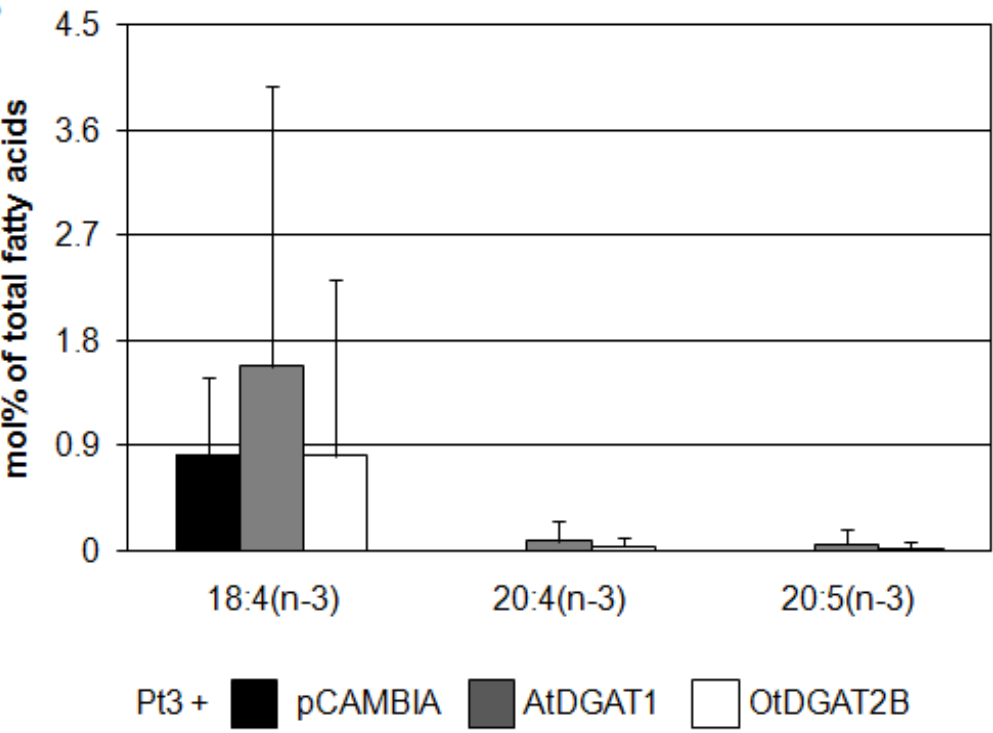

Figure 38. (n-6)- and (n-3)-PUFA contents of transgenic $A$. thaliana tag1-1 seeds co-expressing $\mathrm{Pt} 3$ with AtDGAT1 or OtDGAT2B

Plants were co-transformed with Ptd6-PSE1-Ptd5-pCAMBIA2200 (Pt3) and pCAMBIA33.2cGs, AtDGAT1-pCAMBIA33.2cGs or OtDGAT2B-pCAMBIA33.2cGs. $3 \mathrm{mg}$ T2 seeds were analyzed by pool analysis. (A) (n-6)- PUFA values and (B) (n-3)-PUFA values. Bars represent mean + SD from four to six independent plant lines. 


\section{Discussion}

\subsection{Characterization of microalgal acyltransferases in yeast}

Several nucleotide sequences encoding enzymes putatively involved in VLCPUFA distribution between different lipid fractions were isolated from $O$. lucimarinus and $O$. tauri. Most of the corresponding proteins featured acyltransferase activity upon expression in yeast (Wagner, 2008). The aim of this work was to further investigate their substrate specificities by heterologous expression studies in yeast and to find out whether they are useful candidates to change the fatty acid composition of seed oil.

\subsubsection{OILPCATp and OtLPCATp do not reveal LPLAT activity in vitro}

In a previous study, expression of OILPCAT from O. lucimarinus complemented a yeast mutant deficient in intrinsic LPCAT activity (Wagner, 2008) and was thus a promising candidate for further experiments regarding its substrate specificity. The closely related nucleotide sequence OtLPCAT was isolated recently by Dr. M. Heilmann from $O$. tauri and was also chosen for further investigations. Yeast has been successfully used as heterologous expression host already for the characterization of two LPLAT enzymes from Arabidopsis. Upon expression in yeast, both plant proteins revealed broad LPLAT activity in vitro (Stahl et al., 2008). When the microalgal sequences OILPCAT and OtLPCAT were expressed as V5-tagged versions in yeast, however, proteins with the expected sizes were not detectable in Western Blot (Figure 4). This result was surprising, as in previous studies expression of V5-OILPCAT fusion constructs in the same yeast strain led to sound protein amounts (Wagner, 2008). Expression conditions, protocol and buffers for homogenate preparation as well as amounts of loaded protein were the same as described before (Wagner, 2008), solely a different proteinase inhibitor was used. One possible explanation is that the expressed protein was not stable in yeast. But as in the positive control of the experiment high levels of complete LacZ-V5p and no significant levels of degradation products were detectable (Figure 4), complete protein degradation for OILPCAT and OtLPCAT fusion proteins during homogenate preparation can be ruled out. Another possibility to explain the failure of expression tests, at least for OtLPCAT, is that protein synthesis terminated cotranslationally due to codon usage incompatibilities. Because only the first 14 codons of the sequence were adapted to expression in yeast, inefficient translation of the OtLPCAT mRNA might be a reason for missing protein. In order to rule out that the carboxylterminal tag led to protein degradation, also lysates from cells expressing aminoterminal GFP-tagged versions of OILPCAT and OtLPCAT were assayed by Western Blot and by microscopy. These experiments also did not lead to detectable protein levels after expression of both putative LPCAT nucleotide sequences in yeast. 
Non-detectable protein can be caused by sensitivity issues connected to the used detection method, still expression of OILPCAT and OtLPCAT might have led to functional protein in yeast. Therefore, different LPLAT and thioesterase assays were performed with lysates from expression cultures to investigate the enzymatic activity of OILPCATp and OtLPCATp. The experimental approach to solving the problem of low expression was the usage of untagged nucleotide sequences which might lead to higher protein levels in yeast. Unfortunately, neither thioesterase nor LPLAT activity was detectable in vitro in extracts prepared from cells expressing non-tagged variants of either enzyme, although various combinations of possible acyl donors and acceptors were tested (Table 1). The positive controls were functional, as can be seen from Figure 5 , thus the assay conditions were adequate at least for the yeast LPCAT ALE1p. The latter enzyme has been found to mediate broad LPLAT activity in vitro (Benghezal et al., 2007; Chen et al., 2007; Jain et al., 2007; Riekhof et al., 2007; Tamaki et al., 2007). This was observed also in the presented experiments (Figure 5; Table 1). For in vitro activity of microalgal putative LPCAT proteins, if they were present in the lysates at all, buffer conditions or reaction temperatures might not have been optimal. Maybe also the homogenate preparation procedure influenced their enzymatic activity in a negative manner. Because protein presence in the yeast lysates could not be verified, further optimization attempts of the protocol were not conducted. Due to the described problems, no predictions concerning the effects of expression in planta could be made.

\subsubsection{OtDGAT2Bp prefers 18:1(n-9)-CoA as substrate in vitro}

It has previously been shown that expression of OtDGAT2B complemented a TAGdeficient yeast mutant strain and the encoded protein in vivo accepted polyunsaturated 18- and 20-carbon chain substrates of both the (n-3)- and the (n-6)-series (Wagner, 2008). To verify this rather broad substrate specificity of the microalgal enzyme, the ability of cell homogenates expressing OtDGAT2B, DGA1 or empty vector for TAG formation was tested with various radiolabeled putative acyl donors and two different acyl acceptors. These experiments revealed that in vitro di-18:1(n-9)-DAG as well as di-18:2(n-6)-DAG were accepted by OtDGAT2Bp as acyl acceptors when $\left[1-{ }^{14} \mathrm{C}\right]-$ 18:1(n-9)-CoA was used as acyl donor (Figure 6). In contrast to the results obtained from the in vivo experiments, however, polyunsaturated acyl-CoAs were not used by OtDGAT2Bp, but only by the yeast enzyme DGA1p. For the latter enzyme, also the observed TAG formation activity in vitro was much stronger compared to the microalgal DGAT protein. This could be caused by higher protein amounts upon expression in yeast which was detected before (Wagner, 2008). Furthermore, DGA1p accepted all tested acyl-CoAs with similar preference which reflects also the in vivo obtained results for the protein. As 18:1(n-9)-CoA is an endogenous yeast acyl-CoA, it was not possible to compare the incorporation rate into the TAG fraction of yeast cells expressing OtDGAT2B for 18:1(n-9)-CoA, as it has been done for the polyunsaturated acyl-CoAs with 18 or 20 carbon atoms (Wagner, 2008). Thus, 18:1(n-9)-CoA possibly could also be the preferred substrate for TAG formation by OtDGAT2Bp in vivo. Maybe, in vitro reaction conditions were not optimal for OtDGAT2Bp and led to a strongly reduced 
DGAT activity compared to the situation in vivo. This could explain why no polyunsaturated 18- or 20-carbon chain substrate but only 18:1(n-9)-CoA was accepted by the protein. Shockey and co-workers could show that the tung tree DGAT2 protein also features a distinct substrate preference which strongly influences oil composition of seeds (Shockey et al., 2006). If the OtDGAT2Bp substrate specificity reflected the situation in $O$. tauri, this acyltransferase would not be responsible for the distribution of VLCPUFAs into the microalgal TAG fraction (Wagner et al., 2010). Based on the yeast expression experiments and assuming the catalytic activity of OtDGAT2Bp in planta reflects that in yeast, the data obtained so far do not suggest that the enzyme will substantially contribute to VLCPUFA formation in plants.

\subsubsection{OtDGAT2Ap features a faint TAG formation ability at low expression temperatures}

Previous studies showed that expression of OtDGAT2A and OtDGAT2C in yeast did not lead to TAG formation in a TAG-deficient yeast mutant. Also, feeding experiments with polyunsaturated fatty acids did not enhance DGAT activity of the encoded proteins (Wagner, 2008). Upon lowering the expression temperatures, however, TAG formation could be promoted in case of OtDGAT2Ap (Figure 7). This could be due to elevated protein stability or activity under temperatures which resemble normal growth conditions of the microalgal gene donor organism. Nevertheless, TAG formation observed upon OtDGAT2A expression was not very strong. Following DGAT experiments with homogenates from yeast cells expressing the corresponding sequence did not lead to detectable activity in vitro (Figure 9). This might be caused by growing yeast expression cultures at $30{ }^{\circ} \mathrm{C}$ or suboptimal assay conditions like too high reaction temperatures or inadequate buffers. Furthermore, DGAT activity of OtDGAT2Ap might have been below the assay detection limit, as it was also barely detectable in vivo. This could be also the case for OtDGAT2Cp, whose DGAT activity was neither observable in vivo nor in vitro. Further experiments investigating possible MGAT or WS activities of OtDGAT2Ap or OtDGAT2Cp led to negative results (Figure 8 ). This does not rule out possible roles of both enzymes in $O$. tauri because it maybe only reflects the disability of yeast as an expression host which might not provide the necessary reaction environment for the microalgal enzymes. Also, mislocalization or insufficient posttranslational modifications of the proteins can be a problem for proper functioning of the enzymes in yeast. In some studies investigating DGAT2 activities, insect cells were used for expression of the corresponding cDNAs (Lardizabal et al., 2001). These might possibly be more suitable and could be tested also for expression of microalgal DGAT2 sequences. Thus, in $O$. tauri both proteins could still be important for distribution of VLCPUFAs like DHA into the neutral lipid fraction (Wagner et al., 2010). It should be noted that TAG formation in algae is depending strongly on growth stages and environmental conditions (Bigogno et al., 2002; Khozin-Goldberg et al., 2002) which might influence protein activity or stability indirectly. However, because solid enzymatic 
activity upon heterologous expression in yeast could not be shown so far, both putative DGAT sequences were not considered for plant expression experiments.

\subsubsection{Co-expression of microalgal acyltransferase sequences does not change the enzymatic activity of the encoded proteins}

Man and co-workers could show that co-expression of the stearoyl-desaturase SCD1p and DGAT2 protein from mouse in HeLa cells led to enhanced TAG formation in vivo due to the interaction of both enzymes (Man et al., 2006a). However, co-expression studies with different acyltransferases in yeast have not been performed up to now. In order to test whether the acyltransferases from Ostreococcus display or change their enzymatic activity in yeast upon presence of other acyltransferases from the same organism, it was attempted to co-express OtLPCAT or OILPCAT with OtDGAT2B or OtPDAT. Their effects on acyl-CoA, PC and TAG pool of the yeast expression cultures were investigated subsequently. For the experiments, a yeast mutant lacking endogenous LPCAT, DGAT and PDAT activity was created. This mutant was found to have a different acyl-CoA composition compared to wild type yeast (Figure $10 \mathrm{~B}$ ). Enhanced levels of $16: 1(n-7)$ and lowered amounts of $18: 0$ and $18: 1(n-9)$ in the CoA pool of the yeast mutant cultures might permit conclusions concerning the residual yeast enzymatic activities which influence CoA pool composition like LPLATs or acyl-CoA synthetases. Differences in the total amounts of acyl-CoAs, however, were not significant (Figure $10 \mathrm{~A}$ ). Also the changes in total amounts of PC were not significant and fatty acid composition was the same in the PC fraction of wild type and mutant cells (Figure 12). TAG content was strongly diminished in mutant cells (Figure 11), resulting from the missing DGAT and PDAT activities in the deletion strain.

Co-expression of different combinations of microalgal acyltransferase sequences did not lead to statistically significant differences in the total acyl-CoA amounts and CoA pool composition (Figure 10). Solely, expression of OtDGAT2B led to TAG formation in mutant yeast cultures, independently from the co-expressed sequence (Figure $11 \mathrm{~A}$ ). The fatty acid composition of the neutral lipid fraction, nevertheless, was not changed significantly by the activity of OtDGAT2Bp in comparison to wild type yeast (Figure $11 \mathrm{~B}$ ). The missing TAG formation upon expression of OtPDAT confirmed the results obtained by Dr. Martin Wagner, as also in his experiments TAG formation could not be observed without supplementation of polyunsaturated fatty acids (Wagner, 2008). This enzyme seems to feature a preference for unusual fatty acids, similar to the PDAT1 enzyme from Arabidopsis (Stahl et al., 2004).

Especially concerning total amounts and composition of acyl-CoAs and TAG fraction, great differences could be observed between the independent clones expressing the same combination of acyltransferase sequences which led to high standard deviations. Different expression levels might be the reason for the strong variations between the clones. Due to the previously described expression problems concerning putative microalgal LPCAT sequences, it also cannot be ruled out that OILPCATp or OtLPCATp were not present at all in the yeast expression cultures. Therefore, it cannot be de- 
duced from the presented experiments whether the tested microalgal acyltransferases interact with each other or not in yeast.

\subsection{Characterization of the microalgal desaturase Old4p in yeast}

By seed-specific co-expression of microalgal nucleotide sequences encoding CoAdependent desaturases with $\Delta 6$ - and $\Delta 5$-specificity together with the elongase sequence PSE1 (Zank et al., 2002), for the first time exclusively CoA-dependent EPA production with enzymes originating from the plant kingdom could be established in Arabidopsis (Hoffmann et al., 2008). In order to produce DHA in a CoA-dependent way, the aim of this work was to identify a $\Delta 4$-desaturase with a preference for acyl-CoA substrates. Until now, for this last part of the (n-3)-pathway, three naturally CoAdependent, $\Delta 5$-elongases from microalgal origin have been characterized (Meyer et al., 2004; Pereira et al., 2004b). Also, seven desaturases with $\Delta 4$-specificity were found in microalgae during the past years (Qiu et al., 2001; Meyer et al., 2003; Tonon et al., 2003; Pereira et al., 2004b; Tonon et al., 2005b; Zhou et al., 2007; Liu et al., 2007), but none of them so far has been shown to prefer CoA-bound substrates.

\subsubsection{The genome of $O$. lucimarinus contains a gene coding for a front- end desaturase}

The prasinophyte $O$. lucimarinus was chosen as gene donor, because it is closely related to $O$. tauri and $M$. squamata which both contain CoA-dependent desaturases (Domergue et al., 2005; Hoffmann et al., 2008). Furthermore, the fatty acid composition of $O$. lucimarinus exhibited moderate amounts of the $\Delta 4$-desaturation products DHA and 16:4(n-3) (Figure 13). Although DHA amounts were much lower than in O. tauri (Wagner et al., 2010), they were similar to those found in E. gracilis (Korn, 1964), a microalgal species from which a desaturase with $\Delta 4$-specificity has already been identified a few years ago (Meyer et al., 2003).

The protein sequence of the putative desaturase from $O$. Iucimarinus was found to contain three histidine boxes with an $\mathrm{H}$ to $\mathrm{Q}$ substitution in the last box and an aminoterminally fused cytochrome $b_{5}$ domain (Figure 15). These are typical characteristics of front-end desaturases (Shanklin et al., 1994; Sperling and Heinz, 2001; Domergue et al., 2002; Napier et al., 2003).

\subsubsection{Old4p has a preference for VLCPUFAs and desaturates (n-3)- as well as ( $n-6)$ - substrates with $\Delta 4$-specificity}

When Old4 was expressed in yeast, the encoded protein was found to introduce double bonds at the $\Delta 4$-position and revealed conversion rates of $1 \%$ for $16: 3(n-3), 10 \%$ for $22: 5(n-3)$ and $4 \%$ for 22:4(n-6) as substrates (Figure 17). From these results it was deduced that Old4p prefers VLCPUFAs to shorter-chain substrates and fatty acids of 
the (n-3)-series are favored. The preference for VLCPUFAs is not reflected by the fatty acid profile of 0 . lucimarinus which clearly contains more 16:4(n-3) than DHA. The preference for (n-3)-substrates, in contrast, would correlate with the fact that only (n-3)VLCPUFAs can be found in the total fatty acids of the prasinophyte (Figure 13). To be able to classify the obtained conversion rates, also the previously characterized desaturase sequence Egd4 (Meyer et al., 2003) was expressed in yeast cultures supplemented with the same substrates as those cultures expressing Old4. The observed conversion rates in the yeast strain INVSc1 (15\% for $16: 3(n-3), 12 \%$ for $22: 5(n-3)$ and $4 \%$ for 22:4(n-6) (Figure 17)) were higher and differed substantially from those obtained by Meyer and co-workers. They performed expression studies in the yeast strain 334 (Hovland et al., 1989) and determined conversion rates of $21 \%$ for $16: 3(n-3)$, about $30 \%$ for 22:5(n-3) and $29 \%$ for 22:4(n-6) (Meyer et al., 2003). In contrast to the results in the yeast strain INVSc1, no clear difference between desaturation of (n-3)and ( $n-6)$-substrates was detectable. This example indicates that desaturation rates and substrate preference of a heterologously expressed desaturase sequence do not only depend on the encoded enzyme but also on the expression host. Even though such a situation cannot be conclusively ruled out for the experiments performed here, the data indicate that Old4p might be a suitable candidate enzyme to be further tested in transgenic plants.

\subsubsection{Old4p acts in a lipid-dependent manner}

Because the desaturation product DHA was predominantly present in the PC fraction of yeast cultures expressing Old4 or Egd4 (Figure 18), a lipid-dependent desaturation mechanism may be assumed for both tested desaturases. Acyl-CoA-dependent desaturation, like it has been shown for Otd6p, Msd6p and Msd5p (Domergue et al., 2005; Hoffmann et al., 2008), may have led to an equal distribution of DHA between the lipid classes because the CoA pool constitutes the base for synthesis of all different glycerolipids. For Egd4p, Meyer and co-workers found an accumulation of desaturation pro-ducts at the sn-2 position of PC and thus proposed a positional specificity for lipidbound substrates already in their previous studies (Meyer et al., 2003). For Old4p, nevertheless, a lipid-dependency may be surprising due to the close evolutionary relationship of $O$. Iucimarinus to $O$. tauri. Therefore, additional co-expression studies were performed to further investigate the substrate specificity of the desaturase. Endogenous CoA-substrate levels should be enhanced in yeast by co-expression of a nucleotide sequence from $T$. pseudonana which encodes a LACS protein specialized on VLCPUFAs (Tonon et al., 2005a). If Old4p acted in a CoA-dependent manner, additional expression of the LACS sequence possibly would have boosted desaturation rates. However, co-expression of TpLACS with Old4 in INVSc1 resulted in lower desaturation rates compared to expression of Old4 alone (Figure 19). A possible reason for this could be the accumulation of DPA in the neutral lipid fraction. In their studies, Tonon and co-workers observed that the VLCPUFA content in TAGs of yeast cultures expressing TpLACS was enhanced six times compared to control cultures (Tonon et al., 2005a). This in turn would limit DPA levels in PC, the desaturation substrate for 
lipid-dependent desaturases (Domergue et al., 2003). Nonetheless, Tonon and coworkers only investigated the TAG fraction, but not the phospholipid fractions for presence of VLCPUFAs. In the present study, the amounts of DPA were tested in total lipids and were not found to be enhanced upon expression of TpLACS. However, because expression tests were not performed for TpLACS, it is not clear whether and in which amounts the encoded protein was present in yeast.

In order to test whether glycolipids are the actual substrates of Old4p, co-expression of the corresponding nucleotide sequence was performed with a cucumber MGDG synthase nucleotide sequence (Shimojima et al., 1997) which had been successfully used in co-expression studies with chloroplastidic desaturases from $A$. thaliana in yeast before (Heilmann et al., 2004). Heterologous expression of this sequence led to the production of MGDG in yeast (Figure $20 \mathrm{~A}$ ), but incorporation of externally supplied fatty acid into MGDG was only possible for the substrate 16:3(n-3). Co-expression of Old4 and CsMGDGS, nevertheless, led to a complete lack of desaturation activity in yeast (Figure $20 \mathrm{~B}$ ). As 16:3(n-3) is one of the most prominent fatty acids in plastidial glycolipids (Browse and Somerville, 1991), it might be favored as substrate by the MGDG synthase. Upon expression of CsMGDGS in yeast, this substrate preference could also be the reason for the preferred transfer of 16:3(n-3) into the newly established glycolipid fraction by the MGDG synthase. As Old4p seems to be a PC-dependent desaturase, the potential $\Delta 4$-desaturation substrate in this case would be out of range for the enzyme.

Taken together, co-expression studies suggested that missing substrate in shape of acyl-CoAs or galactolipids were not the limiting factors for desaturation in yeast and supported the assumed substrate preference of Old4p for PC-bound acyl chains. Thus, it could either be that $O$. Iucimarinus and $O$. tauri contain desaturases with different preferences, although they are closely related to each other. Another possibility would be the co-existence of lipid- and acyl-CoA-dependent desaturases in one organism which has not been shown yet. This could be explained for example by gene duplication and subsequent mutations changing the substrate specificities of the corresponding enzymes. Alternatively, the endosymbiotic uptake of bacteria possessing desaturases with other substrate specificities than those of microalgal enzymes in the course of evolution and the subsequent gene transfer into the nucleus could explain this phenomenon.

\subsubsection{Old4p accumulates to solid levels in yeast cells and localizes to their endoplasmic reticulum}

Because desaturation rates in yeast were rather low, protein accumulation upon moderate and strong expression of GFP-Old4 or GFP-Egd4 was examined by Western Blot studies. These revealed comparable and solid amounts of both fusion proteins (Figure 21). The addition of an amino-terminal epitope, nevertheless, is known to enhance steady-state amounts of plant desaturases upon expression in yeast (O'Quin et 
al., 2009). If this is also the case for microalgal desaturases, low protein amounts could still be a problem for untagged versions of Old4p and Egd4p.

Microscopic localization studies were performed to check whether mislocalization or protein aggregation was responsible for the low desaturase activity of Old4p. The GFPtagged version of the desaturase did not form visible aggregates in yeast cells and seemed to localize to the ER (Figure 22). This would be also the expected localization of a microsomal front-end desaturase. But although the localization at first sight appears to be correct, this still does not rule out the possibility of missing microdomain structures in the yeast ER that are required for proper desaturase function. Also other cases of microalgal desaturases with low activity have been reported (Hoffmann et al., 2008; Lu et al., 2009b; Iskandarov et al., 2010). For all of these enzymes, inappropriate posttranslational modifications, wrong substrate compartmentalization and missing cofactors could be possible reasons for low activity.

Taking together the preceding results, the identified $\Delta 4$-desaturase from $O$. lucimarinus was found to act in a lipid-dependent way on (n-3)- as well as (n-6)-substrates with a preference for VLCPUFAs in yeast. However, due to the poor EPA yields obtained so far in Arabidopsis, substrate availability for DHA-producing enzymes in those plants would be rather low. Therefore, the enzyme was not further analyzed in planta.

\subsection{Characterization of acyltransferases, desaturases and elongases in plants}

In previous studies, VLCPUFA production was established in plants but resulted in rather low product yields (Hoffmann et al., 2008). These should be improved by the investigation of additional enzymatic activities required for VLCPUFA distribution between different lipid pools and by testing different combinations of the already known enzymes for primary biosynthesis. Also, different plant species were used for VLCPUFA production to analyze the endogenous effects in the host plant seeds.

\subsubsection{OILPCATp increases levels of polyunsaturated fatty acids in the TAG fraction of Arabidopsis seeds}

After yeast experiments concerning substrate specificities of different acyltransferases, OtDGAT2Bp, OtPDATp, OILPCATp and OtLPCATp were implemented into plant seed lipid metabolism to find out whether they are able to change TAG levels or composition. Thus, the corresponding cDNA sequences were expressed individually under the control of the LeB4 promoter in Arabidopsis seeds. Regarding TAG content, no differences could be observed between transgenic seeds expressing acyltransferase sequences and seeds expressing empty vector (Figure 23). OtDGAT2B expression did also not change the fatty acid composition of total seed lipids (Figure $24 \mathrm{~A}$ ). In view of the obtained in vivo results from yeast which showed no difference between polyunsaturated 18- and 20-carbon fatty acids as substrate for OtDGAT2Bp (Wagner, 2008), strong 
changes in the fatty acid composition of seeds were also not expected in planta upon OtDGAT2B expression. But concerning TAG content, over-expression studies with other fungal or plant DGAT sequences in several plant species including Arabidopsis led to enhanced oil deposition (Jako et al., 2001; Lardizabal et al., 2008; Weselake et al., 2008; Zheng et al., 2008; Taylor et al., 2009). In these studies, seed-specific promoters other than the $L e B 4$ promoter were used which might be a possible reason why expression of OtDGAT2B did not result in a similar effect as it has been observed for the other DGAT sequences.

In previous yeast studies, OtPDATp in vivo showed TAG forming activity only upon supplementation with polyunsaturated 18 - and 20 -carbon chain fatty acids. From these results it was deduced that the enzyme might enhance levels of polyunsaturated fatty acids in the TAG pool also in plant seeds (Wagner, 2008). This assumption could not be confirmed. OtPDAT expression mostly led to small, but significant changes in saturated and monounsaturated fatty acid levels below $1 \%$ in comparison to control seeds, only 20:1(n-9) levels were increased about $2.2 \%$ (Figure 24 B). Also, OtLPCAT expression led to small but significant changes in fatty acid composition (Figure $25 \mathrm{~B}$ ). For both OtPDAT and OtLPCAT expression, more independent T2 plant lines should be examined to find out whether the observed changes are consistent.

A possible reason for the completely missing or at most marginal changes observed upon OtDGAT2B, OtPDAT and OtLPCAT expression could be the fact that they were only expressed as partly codon-optimized versions. This could lead to low protein translation rates and subsequently little observable protein activity. As it is assumed that distinct ER regions are dedicated to TAG formation (Shockey et al., 2006; Cahoon et al., 2007), also mislocalization of the microalgal proteins in planta could be the reason for low activity. Furthermore, missing posttranslational modifications, cofactors or interacting enzymes that are required for proper enzymatic function are a possible explanation. Faint effects could also be caused by the resilience of the host plant metabolism. In case of minor metabolic changes it has been shown that the plant is able to restore the initial seed lipid composition by endogenous feedback mechanisms (Capell and Christou, 2004).

Seeds expressing the completely codon-optimized version of OILPCAT exhibited the strongest effects observed in this experimental setup. They revealed increased levels of the polyunsaturated 18 carbon-chain fatty acids $18: 2(n-6)$ and $18: 3(n-3)$ and a concomitant decrease in 18:1(n-9), the precursor fatty acid of $18: 2(n-6)$ and $18: 3(n-3)$. A similar observation could be made for the 20 carbon-chain fatty acids $20: 1(n-9)$ and 20:2(n-6). Polyunsaturated fatty acid levels were increased and monoenoic fatty acid amounts were decreased in comparison to the control seeds (Figure $25 \mathrm{~A}$ ). LPCAT proteins are believed to mediate the transfer of acyl groups between PC and acyl-CoA pool in both directions. However, Stymne and Stobart showed in safflower cotyledons that the transfer from acyl-CoA pool onto the sn-2 position of lyso-PC is preferred to the back reaction (Stymne and Stobart, 1984). If the same holds true for the putative microalgal LPCAT protein, it would possibly enhance the levels of monounsaturated fatty 
acids at the sn-2 position of PC and thereby increase substrate levels for ER-located desaturases. In turn, these could introduce double bonds into the PC-bound acyl substrates and thus produce polyunsaturated fatty acids. Because the changes in fatty acid compositions were localized in the TAG portion of seeds (Figure 26), however, the produced polyunsaturated fatty acids would need to be transported by other enzymatic activities into the TAG fraction. TAG formation in this case could be accomplished directly via an endogenous Arabidopsis PDAT activity which produces TAG by transferring the acyl chains from the sn-2 position of PC onto the sn-3 position of DAG (Figure 1). Alternatively, CPT or PDCT activities could produce DAG molecules enriched in polyunsaturated fatty acids. These DAG molecules in turn would be used for TAG biosynthesis either by a DGAT or a PDAT activity (Figure 1).

In previous studies, OILPCAT was co-expressed together with Ptd6 and PSE1 in yeast and was found to mediate the transfer of acyl groups also between PC and acyl-CoA pool, by definition the backwards reaction (Wagner, 2008). If in planta this direction would also be preferred by OILPCATp, the enzyme could possibly remove polyunsaturated fatty acids from the $P C$ pool in the seed and enhance their levels in the acyl-CoA pool. The CoA-bound desaturation products could then be incorporated into TAG by an endogenous plant DGAT activity (Figure 1). From the obtained results, it cannot be concluded which direction of acyl transfer the putative LPCATp from O. lucimarinus prefers, because both could in principle be explained with the current TAG biosynthesis model. Nevertheless, the composition of the PC fraction was not affected significantly by expression of OILPCAT (Figure 26). If the polyunsaturated fatty acids are derived from PC, an inverse effect compared to the TAG fraction composition would be expected, resulting in higher levels of monounsaturated fatty acids and lower levels of polyunsaturated fatty acids in this lipid fraction. This effect could again be explained by the resilience of the plant system which leads to generally higher desaturation rates to compensate for the shortfall of unsaturated fatty acids in the membrane lipids. This pronounced adaptation to unforeseen events can also be observed for example as adaptation to lower temperatures in plants. As lowered temperatures lead to more rigid membranes, membrane lipids are desaturated in response to this stimulus to restore membrane fluidity (Ohlrogge and Browse, 1995). A similar effect could be assumed in case of a foreign enzyme activity changing the lipid composition of membrane lipids. It should also be kept in mind that the observed fatty acid composition of total lipids as well as PC and TAG fraction is the final product of all involved endogenous enzymes and the newly introduced enzymatic activity. It would thus be interesting to compare the fatty acid composition of different lipid pools at different time points of seed maturation to find out, which further effects are caused by the foreign enzyme and how the plant responds to it. During these earlier stages, also acyl-CoA composition could be determined to investigate the effects of heterologous OILPCAT expression also in this lipid pool. In summary, the increase of polyunsaturated fatty acids in the seed lipids appeared promising to also test whether co-expression with VLCPUFA-producing enzymes leads to higher yields than the expression of desaturases and elongases alone. 


\subsubsection{The Arabidopsis tag1-1 mutant cannot be rescued by expression of different DGAT nucleotide sequences}

The $A$. thaliana tag 1-1 mutant features strongly reduced TAG content and higher levels of 18:3(n-3) at the expense of 18:1(n-9) and 20:1(n-9). The affected gene has been found to encode the AtDGAT1 protein (Katavic et al., 1995; Routaboul et al., 1999; Zou et al., 1999). The effects concerning overall TAG reduction in comparison to wild type seeds observed by other groups were reproducible when plants were cultivated in the climate chamber (Figure $27 \mathrm{~A}$ ), but not in the greenhouse (Figure 28). Generally, TAG content in wild type seeds was about $100 \mu \mathrm{g} / \mathrm{mg}$ seeds higher upon cultivation in the climate chamber in comparison to the greenhouse. TAG amounts in mutant seeds, however, were equal under the different growth conditions. The difference between both cultivation methods are the more consistent temperature and illumination conditions in the climate chamber, whereas plants are more exposed to seasonal factors in the greenhouse. In case of wild type seeds, the latter growth conditions obviously led to lower TAG accumulation. In case of mutant seeds which already exhibit lowered TAG levels, cultivation conditions did not seem to further influence this factor. Concerning fatty acid composition of wild type and mutant seeds, in contrast, no difference could be observed between climate chamber and greenhouse cultivation. Mutant seeds in both experimental setups revealed the typical flux from the monounsaturated fatty acids $18: 1(n-9)$ and $20: 1(n-9)$ towards the polyunsaturated fatty acid 18:3(n-3) (Figure 27 B and Figure 29). Hence, the fatty acid composition of seed lipids seems to be more independent from the cultivation conditions than the total lipid content.

Surprisingly, expression of the different DGAT nucleotide sequences from Arabidopsis or $O$. tauri under the control of the seed-specific $L e B 4$ promoter did not rescue the mutant phenotype concerning fatty acid composition (Figure 29). At least for the AtDGAT1 sequence, this was expected because complementation also has been shown in previous studies using the seed-specific napin promoter (Jako et al., 2001). There are several possible reasons why the complementation of the mutant was not successful. First, the utilized LeB4 promoter could be weaker and active in different phases than it was anticipated. The promoter has been isolated from Field Bean (Vicia faba) and was shown to drive expression in both Arabidopsis endosperm and embryo (Bäumlein et al., 1991b). Nevertheless, there is no information about promoter activity during different stages of embryo development available in Arabidopsis. Similar studies have been published only for linseed. They showed that the LeB4 promoter is active from 1140 days after flowering (daf) (Drexler et al., 2003). Whether promoter activity is similar in Arabidopsis is not clear. Seed maturation in Arabidopsis leads to the accumulation of storage compounds and acquisition of dormancy and desiccation tolerance (Goldberg et al., 1994). It can be divided into three stages: The first stage lasts from 7-10 days after pollination (dap) and is important for embryo growth and starch accumulation. In the second stage (11-16 dap), starch is degraded again and instead storage proteins as well as fatty acids are synthesized and deposited in the seed which leads to an increase in seed dry weight. During the third phase (17-20 dap), storage compound bio- 
synthesis stops and seeds become quiescent (Baud et al., 2008). For lipid accumulation, thus the second phase is most important. If the promoter activity was the same in Arabidopsis as in linseed, it would match well with this second phase. To test promoter activity in Arabidopsis, fluorescent reporter genes could be fused to the LeB4 promoter and the resulting constructs could be transformed into plants. At different developmental stages, transgenic seeds could then be examined for reporter gene expression. Alternatively, transcription of the acyltransferases under control of the LeB4 promoter could be checked by investigating the mRNA amounts in seeds at different days after pollination.

An alternative approach to complement the observed mutant phenotype would be AtDGAT1 expression under control of the endogenous AtDGAT1 promoter instead of the $L e B 4$ promoter. This would eventually lead to better timed expression. The endogenous promoter could then also be used for expression of AtDGAT2 and OtDGAT2B and thus would lead to more reliable data than the utilization of the LeB4 promoter.

Astonishingly, in those experiments using wild type Arabidopsis plants described in the previous section, effects could be observed upon expression of microalgal acyltransferase sequences under control of the LeB4 promoter. Apart from problems with transcription intensity and timing, hence also mRNA stability could be an issue influencing protein amounts in planta. Inclusion of one or several introns in a gene construct in plants often leads to increased mRNA and subsequent protein accumulation compared to constructs without introns (reviewed in (Koziel et al., 1996; Simpson and Filipowicz, 1996)). In case of the utilized DGAT nucleotide sequences, expression of the gene versions with introns thus might have led to higher protein levels than without introns.

\subsubsection{VLCPUFA amounts in transgenic Arabidopsis seeds are not stable throughout consecutive generations}

Transgenic Arabidopsis T2 and T3 seeds containing different combinations of desaturases with the elongase PSE1p were analyzed for their VLCPUFA content. The obtained data showed that independent plant lines representing independent transformation events did not react in the same way. Half of the analyzed plant lines showed higher VLCPUFA amounts in the next generation, whereas the other half showed lower levels. Standard deviations were also not declining in the following generation (Table 3). In the first selection step, those plant lines with the highest amounts of (n-3)PUFAs were chosen for analysis in the next generation. Most probably, these plant lines did not hold single but multiple insertions. As every insertion segregates separately this leads to strongly varying product yields in the analyzed seeds. Another reason for the differing VLCPUFA amounts could be positional insertion effects. Because insertion of the transformed constructs takes place randomly, fragments can also insert at positions in the genome that are silenced or enhanced in their expression (Kinney et al., 2004). Generally, more independent plant lines with single insertions would need to be tested in order to identify genetically stable plant lines producing solid VLCPUFA amounts throughout consecutive generations. 


\subsubsection{Combination of Msd6p with lipid-dependent desaturases, PSE1p and OILPCATp increases EPA levels in transgenic $A$. thaliana seeds}

Abbadi and co-workers found out that the effects of co-expression of the lipiddependent desaturase sequences Ptd6 and Ptd5 with the CoA-dependent elongase sequence PSE1 in plants are limited by a metabolic bottleneck defined by the transfer of the first pathway intermediate between PC- and acyl-CoA pool (Abbadi et al., 2004). This bottleneck was circumvented in subsequent studies by utilization of the acyl-CoAdependent desaturases Msd6p and Msd5p (Hoffmann et al., 2008). Because of the weak activity of the involved $\Delta 5$-desaturase, nevertheless, EPA yields were quite low in Arabidopsis. Therefore, the lipid-dependent desaturases and the elongase were combined with the acyl-CoA-dependent desaturase Msd6p to test whether the bottleneck identified before was still present or could be shifted to the next step in the metabolic pathway. In a second approach, Ptd6, Ptd5 and PSE1 were co-expressed with OILP$C A T$ to investigate the effect of the putative LPCAT enzyme onto transfer of substrates between PC and CoA pool and the resulting VLCPUFA yields. Unfortunately, upon expression in seeds both constructs did not result in higher amounts of product compared to the $\mathrm{Pt} 3$ construct alone (data not shown). In a third experiment, all enzymatic activities were tested in combination in seeds. This approach resulted in much higher product yields compared to the $\mathrm{Pt} 3$ and the Ms3 constructs tested before (Figure 30). The first intermediate of the pathway, 18:4(n-3), was accumulating at much higher amounts than observed before. This might be caused by the additive effect of the two utilized $\Delta 6$-desaturases. The $\Delta 6$-elongation product was present at levels comparable to those from seeds expressing Ms3 which reflects the improved substrate flux between Msd6p and PSE1p. 20:5(n-3) levels, after all, were six fold enhanced in seeds expressing the OILPCAT-Msd6-Pt3 construct compared to seeds expressing Pt3 or Ms3. This effect could be caused by the higher desaturation activity of Ptd5p compared to Msd5p. In this case, the transfer from CoA pool into PC pool would not be the ratelimiting step in the VLCPUFA biosynthesis pathway. An additional factor potentially influencing product yields is the activity of OILPCATp. This enzyme might enhance transfer rates between different lipid pools and thereby positively influence VLCPUFA yields. However, when both Msd6p and OILPCATp were combined alone with the lipiddependent desaturases and the elongase, none of these effects could be observed. Therefore, the number of tested transgenic lines was either too low for Msd6-Pt3 and OILPCAT-Pt3 or the effects are only achieved when both sequences are combined with the $\mathrm{Pt} 3$ construct. The presented values of $\mathrm{Pt} 3$ and $\mathrm{Ms} 3$ seeds are taken from (Hoffmann et al., 2008). To be able to systematically compare VLCPUFA yields in the diffe-rent transgenic seeds, several independent plant lines expressing Pt3, Ms3 or the respective combination constructs would need to be cultivated in parallel to have the same environmental factors like illumination and temperatures which might also influence product amounts. 


\subsubsection{Camelina is a promising oilseed crop for VLCPUFA production}

The oilseed crop $C$. sativa has not been cultivated extensively for some decades, but recently has experienced a comeback (Lu and Kang, 2008). The reason for this fact is the elevated 18:3(n-3) content in Camelina seeds and the resulting health-promoting high (n-3) over (n-6) ratio (Figure 31). Due to its outstanding 18:3(n-3) levels, the plant species was considered as good choice especially for the production of (n-3)VLCPUFAs and therefore transformed with the VLCPUFA-construct Pt3. Alongside, Arabidopsis seeds expressing the Pt3 construct were analyzed. Results obtained for $A$. thaliana seeds were, especially in the case of T3 seeds, much lower than in the EPA screen described in section 3.3.3 (Table 3, Figure 32 and Figure 33). For both experiments, different plant lines were used. This once again shows the large variation between independent transformation events. Furthermore, the EPA screen results were obtained by pool analysis, whereas in the here described experiments single seeds were analyzed. Analysis of seed pools always gives mean values of a mixture from seeds featuring different numbers of insertions. Every result after single seed analysis, in contrast, is derived just from one seed. Thus, this could also explain the different results obtained by different analysis methods.

Generally, VLCPUFA percentages from total fatty acids were higher in Arabidopsis T2 seeds compared to Camelina T2 seeds (Figure 32). This, however, changed in the subsequent generation, in which Camelina seeds contained higher VLCPUFAs percentages than Arabidopsis seeds in their total fatty acids (Figure 33). This could be due to genetic effects which maybe lead to a more stable integration of constructs into the Camelina genome than into the genome of Arabidopsis. However, one should consider that T3 Camelina seeds looked shriveled and had a much lower content of the most prominent fatty acid 18:3(n-3) in total fatty acids. Also, the initial precursor fatty acid for both (n-3)- and (n-6)-VLCPUFAs, 18:1(n-9), in total fatty acids was lower compared to wild type seeds (Figure 31 and Figure 33). Because the percentages of VLCPUFAs were calculated on the basis of total fatty acids, absolute product amounts in both Arabidopsis and Camelina seeds would need to be determined by utilization of internal standard to be able to compare them.

Although $C$. sativa seed lipids have a higher $(n-3)$ over $(n-6)$ ratio than $A$. thaliana seed lipids (Figure 31), much more (n-6)-VLCPUFAs than (n-3)-VLCPUFAs were produced in Camelina seeds (Figure 32 and Figure 33). This observation could be explained by differences in substrate availability. The lipid-dependent desaturase Ptd6p requires PCbound substrates for its enzymatic activity (Domergue et al., 2003). If more 18:2(n-6) than $18: 3(n-3)$ is available in the PC pool, this in turn would lead to higher product amounts of the (n-6)-series. 18:3(n-3), on the other hand, might be transferred directly after its synthesis into the TAG fraction and thus would not be available for further enzymatic activities.

From the presented results it can be deduced that in principle VLCPUFA production in the oilseed crop $C$. sativa is possible and leads to reasonable product yields. Nevertheless, transformation efficiency for this plant species was quite low. That is why only a 
few independent plant lines could be analyzed. More lines and constructs with different promoters and genes would need to be tested in consecutive generations to find out which combinations are most effective.

\subsubsection{Combination of microalgal acyltransferases and primary VLCPUFA biosynthesis activities does not lead to higher product yields}

After the independent analysis of acyltransferases and VLCPUFA-producing enzymes in planta, combinations of both were tested in Arabidopsis wild type as well as tag1-1 seeds. Due to the transformation of two different constructs which inserted separately into the plant genome, two sequential selection steps were required. This only led to a small number of surviving plants for subsequent analysis. Large variations in VLCPUFA yields were observable for independent plant lines and might be caused by multiple insertions or positional effects of Pt3 insertion. TAG contents were not affected by co-expression of acyltransferase sequences with Pt3 in both wild type and mutant seeds (Figure 34 and Figure 37). Resulting VLCPUFA yields were also not different in seeds co-expressing acyltransferases compared to those expressing empty vector with the Pt3 construct. This can have several reasons. First, the issues discussed for acyltransferases in the previous sections hold true in the same way. Furthermore, in both constructs, different promoters were used. Whereas the LeB4 promoter was driving expression of acyltransferase sequences, the USP promoter was used to drive expression of Ptd6, PSE1 and Ptd5. The latter one has also been isolated from V. faba and was shown to be expressed in the Arabidopsis embryo (Bäumlein et al., 1991a). In linseed, the USP promoter was found to be active during 5-40 daf, six days earlier than the LeB4 promoter (Drexler et al., 2003). If the same holds true for expression in Arabidopsis, both promoters should have a long-lasting overlapping activity and thus should be suitable for combined utilization. If in Arabidopsis, however, expression activity of both promoters would be different than in linseed, this could be a possible reason for the missing effect of acyltransferases onto VLCPUFA yields.

In previous studies it was shown that the desaturase SCD1p and the acyltransferase DGAT2p in vivo interact with each other (Man et al., 2006a). The enzymes from $O$. tauri and $O$. lucimarinus, in contrast, most probably do not interact with the desaturases from $P$. tricornutum. This could be the reason for the absent effect of co-expression. Utilization of enzymatic activities from one gene donor, on the other hand, might possibly have led to an observable increase in VLCPUFA yields.

In case of the tag1-1 mutant seeds which feature higher 18:3(n-3) levels than wild type seeds (Figure $27 \mathrm{~B}$ ), higher amounts of (n-3)-VLCPUFAs were expected than in the wild type. These were, similarly to the situation in Camelina seeds, not observable. The reasons for this result could be the same as discussed above for Camelina. Maybe, substrate availability, but not total amounts of the relevant fatty acid are rate-limiting for further modifications. 


\subsection{Future perspectives}

Since the first attempts about 15 years ago, fundamental progress has been made towards the aim of VLCPUFA production in plants. At the same time, also a deeper insight into seed lipid metabolism has evolved. Nevertheless, not all processes occurring during seed maturation and synthesis as well as distribution of unusual fatty acids are as yet fully understood. As an example, recently a completely new enzymatic activity was identified to play a key role in seed lipid metabolism by transferring the headgroup of PC onto DAG and vice versa (Figure 1) (Lu et al., 2009a). As long as the seed lipid metabolism is not completely known, it is of course difficult to find the right switch to change it according to the corresponding needs. Absolute yields might be increased by a metabolic pull function which distributes products into storage lipids, where they are not as strongly accessible by further enzymatic activities than in other pools (Cahoon et al., 2007). This metabolic pull function needs to be highly specific to not transfer already intermediates of the production pathway, but only the end product. DGATs or PDATs with a preference for EPA or DHA for example would be helpful tools to improve the metabolic flux of substrates through the VLCPUFA pathway. These enzymes might be identified by investigating lipid composition and fatty acid distribution into different lipid fractions from potential candidate organisms. The expression pattern of the genes of interest should be investigated in-depth, especially in phases, in which changes in the fatty acid composition or distribution become visible. This may allow conclusions concerning the substrate specificity of the enzymes in question.

As the acyl-CoA pool plays a central role in lipid metabolism, it is also of vital importance for the production of unusual fatty acids. In plants, this lipid pool is assumed to be rather low compared to yeast or animals (Larson and Graham, 2001). Therefore, enhancing the flux of fatty acids into this pool might be a useful means to enhance product yields (Venegas-Calerón et al., 2010). However, because also in this respect the current understanding is rather limited and the current model of direct export of products from the plastidial FAS complex into the cytosolic CoA pool has been questioned recently (Bates et al., 2007), further research is required also in this field of lipid metabolism (Venegas-Calerón et al., 2010).

The transfer of substrates between acyl-CoA pool and PC pool represents a bottleneck in VLCPUFA-production in plants (Abbadi et al., 2004). A solution for this problem would be the identification of a LPCAT enzyme specific for the substrates of VLCPUFA-biosynthesis. Alternatively, highly active acyl-CoA-dependent desaturases with $\Delta 5$ - and $\Delta 4$-specificity would need to be identified to circumvent the described bottleneck.

Clearly, VLCPUFA-biosynthesis in plants has to be accomplished by a concerted expression of several primary and secondary enzymatic activities that need to cooperate in the host organism. This requires utilization of balanced promoters which are active during the phase of seed maturation to assure optimal product yields. To find out, which promoters are suited best for this goal, large-scale promoter studies need to be 
performed. These need to be done for every potential VLCPUFA-producing host species independently, because promoter activity might vary between different organisms. As it is known that using the same promoter several times in one construct can lead to silencing effects, adequate combinations of different promoters have to be identified. Currently, the promoters used for seed-specific expression of genes are derived from storage protein synthesis. It might also be worth to isolate promoters involved in lipid synthesis and oil accumulation during seed development (Venegas-Calerón et al., 2010).

Upon expression of foreign genes in a heterologous system, compartmentalization of substrates and enzymes turned out to be problematic (Graham et al., 2007). It was also proposed that there are several CoA pools in the cell which are not accessible by all lipid-modifying enzymes (Dyer and Mullen, 2008). These issues further complicate the production of a desired fatty acid product in huge amounts. For optimal product yields, introduced enzymatic activities need to be co-localized to have an optimal metabolic flux through the biosynthesis pathway, as it has for example been realized by nature for the FAS complex or the PKS-like system. Investigating these complex structures might lead to transferable solutions to overcome the presented problems. 


\section{Summary and Conclusions}

The production of dietary important VLCPUFAs like EPA or DHA in annual oilseed crops represents a valuable substitute for fish stocks as declining natural sources of these fatty acids. However, there are still hurdles which need to be overcome, especially concerning absolute VLCPUFA yields and distribution of the end products into the neutral lipid fraction of seeds. The implementation of specific acyltransferases and highly active desaturases from primary producers of VLCPUFAs might help to optimize these processes.

Several nucleotide sequences encoding potential acyltransferases from 0 . lucimarinus and $O$. tauri were characterized by in vivo and in vitro experiments in yeast. Two microalgal DGAT enzymes, OtDGAT2Ap and OtDGAT2Bp, were found to complement a yeast mutant deficient in TAG synthesis. OtDGAT2Ap was only active at expression temperatures between $16{ }^{\circ} \mathrm{C}$ and $23{ }^{\circ} \mathrm{C}$, whereas OtDGAT2Bp produced TAG also upon expression at $30{ }^{\circ} \mathrm{C}$. The latter was the only DGAT enzyme revealing activity in vitro. It preferred monounsaturated 18 carbon-chain fatty acids as substrate and did not show any activity towards VLCPUFAs.

In addition, the nucleotide sequence Old4 encoding a front-end desaturase with $\Delta 4$ specificity was isolated from the microalgal species $O$. lucimarinus and characterized by heterologous expression in yeast. The enzyme Old4p was found to desaturate substrates of the (n-3)- and the (n-6)-series in a lipid-dependent way and preferred VLCPUFAs to shorter-chain fatty acids.

Those acyltransferases with the strongest activity, demonstrated in preceding yeast experiments, were introduced individually and in combination with VLCPUFA-producing enzymes into $A$. thaliana to investigate their interplay with seed metabolism. Upon expression of the microalgal nucleotide sequence OILPCAT from O. Iucimarinus, absolute amounts of polyunsaturated fatty acids were elevated in the TAG fraction of seeds. In contrast, expression of all other tested acyltransferase sequences did not result in a change of fatty acid composition in seeds. Further co-expression studies with sequences encoding enzymes necessary for VLCPUFA-biosynthesis did not show a positive effect of acyltransferases on VLCPUFA yields. Besides the analysis of microalgal acyltransferases in planta, with $A$. thaliana and $C$. sativa also two different host plant species were investigated for VLCPUFA-production to compare the effects of endogenous acyltransferases onto product yields. In these studies, Camelina was identified as a promising oilseed crop for heterologous biosynthesis of VLCPUFAs. 


\section{Bibliography}

Abbadi A, Domergue F, Bauer J, Napier JA, Welti R, Zähringer U, Cirpus P, Heinz E (2004) Biosynthesis of Very-Long-Chain Polyunsaturated Fatty Acids in Transgenic Oilseeds: Constraints on Their Accumulation. Plant Cell 16: 2734-2748

Abbadi A, Fulda M, Feussner I, Orsini J, Weyen J, Frauen M, Leckband G (2007) Genetic engineering and breeding of long-chain polyunsaturated fatty acids (LCPUFA) in rapeseed. In J Meng, C Lu, J Zhao, C Liu, W Hua, eds, Proceedings - The 12th International Rapeseed Congress Sustainable Development in Cruciferous Oilseed Crops Production - Vol. II Biotechnology, Vol II. Science Press USA Inc., Monmouth Junction, NJ, USA, pp $152-155$

Aitzetmüller K, Tsevegsüren N (1994) Seed fatty acids, 'front-end'-desaturases and chemotaxonomy - a case study in the ranunculaceae. J Plant Physiol 143: 538-543

Alonso DL, Maroto FG (2000) Plants as 'chemical factories' for the production of polyunsaturated fatty acids. Biotechnology Advances 18: 481-497

Altschul SF, Madden TL, Schäffer AA, Zhang J, Zhang Z, Miller W, Lipman JD (1997) Gapped BLAST and PSI-BLAST: a new generation of protein database search programs. Nucleic Acids Research 25: 389-3402

Arondel V, Lemieux B, Hwang I, Gibson S, Goodman H, Somerville C (1992) Map-based cloning of a gene controlling omega-3 fatty acid desaturation in Arabidopsis. Science 258: $1353-1355$

Ausubel FM, Brent RE, Kingston DD, Seidmann JR, Smith JA, Struhl K (1993) Current Protocols in Molecular Biology. Green Publishing Associates and John Wiley and Sons Inc., New York

Bates PD, Durrett TP, Ohlrogge JB, Pollard M (2009) Analysis of Acyl Fluxes through Multiple Pathways of Triacylglycerol Synthesis in Developing Soybean Embryos. Plant Physiology 150: 55-72

Bates PD, Ohlrogge JB, Pollard M (2007) Incorporation of Newly Synthesized Fatty Acids into Cytosolic Glycerolipids in Pea Leaves Occurs via Acyl Editing. J. Biol. Chem. 282: 31206-31216

Baud Sb, Dubreucq B, Miquel M, Rochat C, Lepiniec Lc (2008) Storage Reserve Accumulation in Arabidopsis: Metabolic and Developmental Control of Seed Filling. In The Arabidopsis Book. The American Society of Plant Biologists, pp 1-24

Bäumlein H, Boerjan W, Nagy I, Bassuner R, Van Montagu M, Inze D, Wobus U (1991a) A novel seed protein gene from Vicia faba is developmentally regulated in transgenic tobacco and Arabidopsis plants. Mol Gen Genet 225: 459-467.

Bäumlein H, Boerjan W, Nagy I, Panitz R, Inze D, Wobus U (1991b) Upstream sequences regulating legumin gene expression in heterologous transgenic plants. Mol Gen Genet 225: $121-128$

Beaudoin F, Michaelson LV, Hey SJ, Lewis MJ, Shewry PR, Sayanova O, Napier JA (2000) Heterologous reconstitution in yeast of the polyunsaturated fatty acid biosynthetic pathway. Proceedings of the National Academy of Sciences USA 97: 6421-6426

Benghezal M, Roubaty C, Veepuri V, Knudsen J, Conzelmann A (2007) SLC1 and SLC4 Encode Partially Redundant Acyl-Coenzyme A 1-Acylglycerol-3-phosphate OAcyltransferases of Budding Yeast. J. Biol. Chem. 282: 30845-30855

Berg J, Tymoczko J, Stryer L (2004) Biochemistry, Ed 5th ed. WH Freeman, New York 
Bigogno C, Khozin-Goldberg I, Cohen Z (2002) Accumulation of arachidonic acid rich triacylglycerols in the microalga Parietochloris incisa (Trebuxiophyceae, Chlorophyta). Phytochemistry 60: 135-143

Bloomfield DK, Bloch K (1960) The formation of delta9-unsaturated fatty acids. J. Biol. Chem. 235: $337-345$

Bouvier-Nave P, Benveniste P, Oelkers P, Sturley SL, Schaller H (2000) Expression in yeast and tobacco of plant cDNAs encoding acyl CoA:diacylglycerol acyltransferase. Eur J Biochem 267: 85-96

Brachmann CB, Davies A, Cost GJ, Caputo E, Li J, Hieter P, Boeke JD (1998) Designer deletion strains derived from Saccharomyces cerevisiae S288C: a useful set of strains and plasmids for PCR-mediated gene disruption and other applications. Yeast 14: 115132

Bradford MM (1976) A rapid and sensitive method for the quantitation of microgram quantities of proteins utilizing the principle of protein-dye binding. Anal. Biochem. 72: 248-254

Brenna JT (2002) Efficiency of conversion of alpha-linolenic acid to long chain n-3 fatty acids in man. Curr Opin Clin Nutr Metab Care 5: 127-132

Brough CL, Coventry JM, Christie WW, Kroon JTM, Brown AP, Barsby TL, Slabas AR (1996) Towards the genetic engineering of triacylglycerols of defined fatty acid composition: major changes in erucic acid content at the sn-2 position affected by the introduction of a 1-acyl-sn-glycerol-3-phosphate acyltransferase from Limnanthes douglasii into oil seed rape. Molecular Breeding 2: 133-142

Broun P, Shanklin J, Whittle E, Somerville C (1998) Catalytic plasticity of fatty acid modification enzymes underlying chemical diversity of plant lipids. Science 282: 1315-1317

Browse J, Somerville C (1991) Glycerolipid synthesis - Biochemistry and regulation. Annual Review of Plant Physiology and Plant Molecular Biology 42: 467-506

Browse J, Warwick N, Somerville CR, Slack CR (1986) Fluxes through the prokaryotic and eukaryotic pathways of lipid synthesis in the '16:3' plant Arabidopsis thaliana. Biochem J 235: 25-31

Buchanan BB, Gruissem W, Jones RL (2000) Biochemistry \& Molecular Biology of Plants. American Society of Plant Biologists, Rockville, Maryland

Bullock WD, Fernandez JM, Hort JMS (1987) XL1-Blue: A high efficiency plasmid transformants recA Escherichia coli strain $\beta$-galactosidase selection. BioTechniques 5: 376-378

Burgal J, Shockey J, Lu C, Dyer J, Larson T, Graham I, Browse J (2008) Metabolic engineering of hydroxy fatty acid production in plants: RcDGAT2 drives dramatic increases in ricinoleate levels in seed oil. Plant Biotechnology Journal 6: 819-831

Cahoon EB, Shockey JM, Dietrich CR, Gidda SK, Mullen RT, Dyer JM (2007) Engineering oilseeds for sustainable production of industrial and nutritional feedstocks: solving bottlenecks in fatty acid flux. Current Opinion in Plant Biology 10: 236-244

Calder PC (2004) N-3 fatty acids and cardiovascular disease: evidence explained and mechanisms explored. Clin. Sci. 107: 1-11

Capell T, Christou P (2004) Progress in plant metabolic engineering. Current Opinion in Biotechnology 15: 148-154

Carlson S, Werkman S, Peeples J, Cooke R, Tolley E (1993) Arachidonic Acid Status Correlates with First Year Growth in Preterm Infants. Proc Nat Acad Sci USA 90: 1073-1077

Chen Q, Kazachkov M, Zheng Z, Zou J (2007) The yeast acylglycerol acyltransferase LCA1 is a key component of Lands cycle for phosphatidylcholine turnover. FEBS Letters 581: 5511-5516

Chen X, Hyatt BA, Mucenski ML, Mason RJ, Shannon JM (2006) Identification and characterization of a lysophosphatidylcholine acyltransferase in alveolar type II cells. PNAS 103: 11724-11729 
Cheng B, Wu G, Vrinten P, Falk K, Bauer J, Qiu X (2010) Towards the production of high levels of eicosapentaenoic acid in transgenic plants: the effects of different host species, genes and promoters. Transgenic Research 19: 221-229

Cheng JB, Russell DW (2004a) Mammalian wax biosynthesis: I. Identification of two fatty acylCoA reductases with different substrate specificities and tissue distributions. J. Biol. Chem. 279: 37789-37797

Cheng JB, Russell DW (2004b) Mammalian Wax Biosynthesis: II. Expression cloning of wax synthase cDNAs encoding a member of the acyltransferase enzyme family. J. Biol. Chem. 279: 37798-37807

Clough SJ, Bent AF (1998) Floral dip: a simplified method for Agrobacterium-mediated transformation of Arabidopsis thaliana. Plant J 16: 735-743

Corpet F (1988) Multiple sequence alignment with hierarchical clustering. Nucleic Acids Res 16: $10881-10890$

Crawford M, Costeloe K, Ghebremeskel K, Phylactos A, Skirvin L, Stacey F (1997) Are deficits of arachidonic and docosahexaenoic acids responsible for the neural and vascular complications of preterm babies? Am J Clin Nutr 66: 1032-1041

Czabany T, Wagner A, Zweytick D, Lohner K, Leitner E, Ingolic E, Daum G (2008) Structural and biochemical properties of lipid particles from the yeast Saccharomyces cerevisiae. J. Biol. Chem. 283: 17065-17074

Dahlqvist A, Banas A, Stymne S, eds (1998) Selective channeling of unusual fatty acids into triacylglycerols. Universidad de Sevilla, Sevilla

Dahlqvist A, Stahl U, Lenman M, Banas A, Lee M, Sandager L, Ronne H, Stymne S (2000) Phospholipid:diacylglycerol acyltransferase: An enzyme that catalyzes the acyl-CoAindependent formation of triacylglycerol in yeast and plants. Proc Natl Acad Sci U S A 97: 6487-6492

Dailey HA, Strittmatter P (1979) Modification and identification of cytochrome b5 carboxyl groups involved in protein-protein interaction with cytochrome b5 reductase. J. Biol. Chem. 254: 5388-5396

Damude HG, Zhang H, Farrall L, Ripp KG, Tomb J-F, Hollerbach D, Yadav NS (2006) Identification of bifunctional $\Delta 12 / \omega 3$ fatty acid desaturases for improving the ratio of $\omega 3$ to $\omega 6$ fatty acids in microbes and plants. PNAS 103: 9446-9451

Demaison L, Moreau D (2002) Dietary n-3 polyunsaturated fatty acids and coronary heart disease-related mortality: a possible mechanism of action. Cell Mol Life Sci 59: 463-477

Diaz AR, Mansilla MC, Vila AJ, de Mendoza D (2002) Membrane topology of the acyl-lipid desaturase from Bacillus subtilis. J. Biol. Chem. 277: 48099-48106

Domergue F, Abbadi A, Ott C, Zank TK, Zahringer U, Heinz E (2003) Acyl carriers used as substrates by the desaturases and elongases involved in very long-chain polyunsaturated fatty acids biosynthesis reconstituted in yeast. J Biol Chem 278: 35115-35126

Domergue F, Abbadi A, Zähringer U, Moreau H, Heinz E (2005) In vivo-characterisation of the first acyl-CoA $\Delta 6$-desaturase from the plant kingdom. Biochem J 389: 483-490

Domergue F, Lerchl J, Zähringer U, Heinz E (2002) Cloning and functional characterization of Phaeodactylum tricornutum front-end desaturases involved in eicosapentaenoic acid biosynthesis. Eur J Biochem 269: 4105-4113

Donahue TF, Cigan AM (1990) Sequence and structural requirements for efficient translation in yeast. Methods Enzymol 185: 366-372

Drexler HHS, Scheffler JA, Heinz E (2003) Evaluation of putative seed-specific promoters for Linum usitatissimum. Molecular Breeding 11: 149-158

Dyer JM, Chapital DC, Kuan JC, Mullen RT, Turner C, McKeon TA, Pepperman AB (2002) Molecular analysis of a bifunctional fatty acid conjugase/desaturase from tung. Implications for the evolution of plant fatty acid diversity. Plant Physiol 130: 2027-2038 
Dyer JM, Mullen RT (2008) Engineering plant oils as high-value industrial feedstocks for biorefining: the need for underpinning cell biology research. Physiologia Plantarum 132: 1122

Ejsing CS, Sampaio JL, Surendranath V, Duchoslav E, Ekroos K, Klemm RW, Simons K, Shevchenko A (2009) Global analysis of the yeast lipidome by quantitative shotgun mass spectrometry. Proceedings of the National Academy of Sciences 106: 2136-2141

Emanuelsson O, Nielsen H, von Heijne G (1999) ChloroP, a neural network-based method for predicting chloroplast transit peptides and their cleavage sites. Protein Science 8: 978984

Fehling E, Lessire R, Cassagne C, Mukherjee KD (1992) Solubilization and partial purification of constituents of acyl-CoA elongase from Lunaria annua. Biochimica et Biophysica Acta (BBA) - Lipids and Lipid Metabolism 1126: 88-94

Felsenstein J (2005) PHYLIP (phylogeny inference package). In. Department of Genome Sciences, University of Washington, Seattle (WA)

Folch J, Lees M, Stanley GHS (1957) A simple method for the isolation and purification of total lipides from animal tissues. J Biol Chem 266: 497-509

Fulco AJ (1974) Metabolic Alterations of Fatty Acids. Annual Review of Biochemistry 43: 215241

Fulda M, Heinz E, Wolter FP (1997) Brassica napus cDNAs encoding fatty acyl-CoA synthetase. Plant Molecular Biology 33: 911-922

Funk CD (2001) Prostaglandins and leukotrienes: advances in eicosanoid biology. Science 294: $1871-1875$

Galili G, Galili S, Lewinsohn E, Tadmor Y (2002) Genetic, molecular and genomic approaches to improve the value of plant foods and feeds. Crit Rev Plant Sci 21: 167-204

Galle-Le Bastard AM, Demandre C, Oursel A, Joseph M, Mazliak P, Kader JC (2000) Phosphatidylcholine molecular species involved in $\gamma$-linolenic acid biosynthesis in microsomes from borage seeds. Physiologia Plantarum 108: 118-124

Gao J, Ajjawi I, Manoli A, Sawin A, Xu C, Froehlich JE, Last RL, Benning C (2009) FATTY ACID DESATURASE4 of Arabidopsis encodes a protein distinct from characterized fatty acid desaturases. The Plant Journal 60: 832-839

Gasteiger E, Hoogland C, Gattiker A, Duvaud Se, Wilkins MR, Appel RD, Bairoch A (2005) Protein Identification and Analysis Tools on the ExPASy Server. In The Proteomics Protocols Handbook. Humana Press, pp 571-607

Gerster H (1998) Can adults adequately convert alpha-linolenic acid (18:3n-3) to eicosapentaenoic acid (20:5n-3) and docosahexaenoic acid (22:6n-3)? Int J Vitam Nutr Res 68: 159-173

Giusto NM, Pasquare SJ, Salvador I., Roque ME, Ilincheta de Boschero MG (2000) Lipid metabolism in vertebrate retinal rod outer segments. Prog. Lipid Res. 39: 315-391

Glomset JA (2006) Role of Docosahexaenoic Acid in Neuronal Plasma Membranes. Sci. STKE 2006: pe6-

Goldberg RB, de Paiva G, Yadegari R (1994) Plant Embryogenesis: Zygote to Seed. Science 266: $605-614$

Goldstein AL, McCusker JH (1999) Three new dominant drug resistance cassettes for gene disruption in Saccharomyces cerevisiae. In, Vol 15. John Wiley \& Sons, Ltd., pp 15411553

Goyens PL, Spilker ME, Zock PL, Katan MB, Mensink RP (2006) Conversion of alphalinolenic acid in humans is influenced by the absolute amounts of alpha-linolenic acid and linoleic acid in the diet and not by their ratio. Am J Clin Nutr 84: 44-53

Graham IA, Larson T, Napier JA (2007) Rational metabolic engineering of transgenic plants for biosynthesis of omega-3 polyunsaturates. Current Opinion in Biotechnology 18: 142147 
Guillard R, Hargraves, PE (1993) Stichochrysis immobilis is a diatom, not a chrysophyte. Phycologia 32: 234-236

Guillard RR, Ryther JH (1962) Studies of marine planktonic diatoms. I. Cyclotella nana Hustedt, and Detonula confervacea (cleve) Gran. Can J Microbiol 8: 229-239

Guldener U, Heck S, Fiedler T, Beinhauer J, Hegemann JH (1996) A New Efficient Gene Disruption Cassette for Repeated Use in Budding Yeast. Nucleic Acids Research 24: 2519-2524

Hackett C, Strittmatter P (1984) Covalent cross-linking of the active sites of vesicle-bound cytochrome b5 and NADH-cytochrome b5 reductase. J. Biol. Chem. 259: 3275-3282

Harwood JL (1996) Recent advances in the biosynthesis of plant fatty acids. Biochimica et Biophysica Acta (BBA) - Lipids and Lipid Metabolism 1301: 7-56

Hastings N, Agaba M, Tocher DR, Leaver MJ, Dick JR, Sargent JR, Teale AJ (2001) A vertebrate fatty acid desaturase with $\Delta 5$ and $\Delta 6$ activities. PNAS 98: 14304-14309

He X, Turner C, Chen GQ, Lin JT, McKeon TA (2004) Cloning and characterization of a cDNA encoding diacylglycerol acyltransferase from castor bean. Lipids 39: 311-318

Heilmann I, Pidkowich MS, Girke T, Shanklin J (2004) Switching desaturase enzyme specificity by alternate subcellular targeting. Proceedings of the National Acadcademy of Sciences USA 101: 10266-10271

Heinz E (1993) Biosynthesis of polyunsaturated fatty acids. In TS Moore, Jr., ed, Lipid Metabolism in Plants. CRC Press, London, pp 33-89

Hellens R, Mullineaux P, Klee H (2000) Technical Focus:A guide to Agrobacterium binary Ti vectors. Trends in Plant Science 5: 446-451

Hites RA, Foran JA, Carpenter DO, Hamilton MC, Knuth BA, Schwager SJ (2004) Global Assessment of Organic Contaminants in Farmed Salmon. Science 303: 226-229

Hobbs DH, Lu C, Hills MJ (1999) Cloning of a cDNA encoding diacylglycerol acyltransefrase from Arabidopsis thaliana and its functional expression. FEBS Letters 452: 145-149

Hoffmann M (2008) Ein neuer Syntheseweg für wertvolle Fettsäuren in Saccharomyces cerevisiae und Arabidopsis thaliana Georg August University, Goettingen

Hoffmann M, Hornung E, Busch S, Kassner N, Ternes P, Braus GH, Feussner I (2007) A small membrane-peripheral region close to the active center determines regioselectivity of membrane-bound fatty acid desaturases from Aspergillus nidulans. J. Biol. Chem. 282: $26666-26674$

Hoffmann M, Wagner M, Abbadi A, Fulda M, Feussner I (2008) Metabolic engineering of $\omega 3$ very long chain polyunsaturated fatty acid production by an exclusively acyl-CoAdependent pathway. J. Biol. Chem. 283: 22352-22362

Höfgen R, Willmitzer L (1988) Storage of competent cells for agrobacterium transformation. Nucleic Acids Research 16: 9877

Hofmann K (2000) A superfamily of membrane-bound O-acyltransferases with implications for Wnt signaling. Trends in Biochemical Sciences 25: 111-112

Hood EE, Gelvin SB, Melchers LS, Hoekema A (1993) New Agrobacterium helper plasmids for gene transfer to plants. Transgenic Research 2: 208-218

Hoppe K (2008) The production of VLCPUFAs in algae and yeast. Georg August University, Goettingen

Hornung E, Pernstich C, Feussner I (2002) Formation of conjugated $\Delta^{11} \Delta^{13}$-double bonds by $\Delta^{12}$-linoleic acid $(1,4)$-acyl-lipid-desaturase in pomegranate seeds. Eur J Biochem 269: 4852-4859

Horton P, Park K-J, Obayashi T, Fujita N, Harada H, Adams-Collier CJ, Nakai K (2007) WoLF PSORT: protein localization predictor. Nucleic Acids Research 35: W585-W587

Hovland P, Flick J, Johnston M, Sclafani RA (1989) Galactose as a gratuitous inducer of GAL gene expression in yeasts growing on glucose. Gene 83: 57-64 
Hwang D (2000) Fatty acids and immune responses-a new perspective in searching for clues to mechanism. Annual Review of Nutrition 20: 431-456

Inoue H, Nojima H, Okayama H (1990) High efficiency transformation of Escherichia coli with plasmids. Gene 96: 23-28

Irazú CE, González-Rodríguez S, Brenner RR (1993) $\Delta 5$-desaturase activity in rat kidney microsomes. Molecular and Cellular Biochemistry 129

Iskandarov U, Khozin-Goldberg I, Cohen Z (2010) Identification and Characterization of $\Delta 12$, $\Delta 6$, and $\Delta 5$ Desaturases from the Green Microalga Parietochloris incisa. Lipids 45: 519530

Ito H, Fukuda Y, Murata K, Kimura A (1983) Transformation of intact yeast cells treated with alkali cations. J Bacteriol 153: 163-168

Itoh R, Toda K, Takahashi H, Takano H, Kuroiwa T (1998) Delta-9 fatty acid desaturase gene containing a carboxyl-terminal cytochrome b5 domain from the red alga Cyanidioschyzon merolae. Curr Genet 33: 165-170

Jackson FM, Fraser TCM, Smith MA, Lazarus C, Stobart AK, Griffiths G (1998) Biosynthesis of $\mathrm{C}_{18}$ polyunsaturated fatty acids in microsomal membrane preparations from the filamentous fungus Mucor circinelloides. European Journal of Biochemistry 252: 513519

Jacobson BS, Jaworski JG, Stumpf PK (1974) Fat metabolism in higher plants: LXII. stearoyl-acyl carrier protein desaturase from spinach chloroplasts. Plant Physiol. 54: 484-486

Jain S, Stanford N, Bhagwat N, Seiler B, Costanzo M, Boone C, Oelkers P (2007) Identification of a Novel Lysophospholipid Acyltransferase in Saccharomyces cerevisiae. J. Biol. Chem. 282: 30562-30569

Jako C, Kumar A, Wei Y, Zou J, Barton DL, Giblin EM, Covello PS, Taylor DC (2001) Seedspecific over-expression of an Arabidopsis cDNA encoding a diacylglycerol acyltransferase enhances seed oil content and seed weight. Plant Physiol 126: 861-874.

Jakobsson A, Westerberg R, Jacobsson A (2006) Fatty acid elongases in mammals: Their regulation and roles in metabolism. Progress in Lipid Research 45: 237-249

Jaworski JG, Stumpf P. K. (1974) Fat metabolism in higher plants: properties of a soluble stearoyl-acyl carrier protein desaturase from maturing Carthamus tinctorius. Arch Biochem Biophys 162: 158-165

Jenke-Kodama H, Sandmann A, Mueller R, Dittmann E (2005) Evolutionary Implications of Bacterial Polyketide Synthases. Molecular Biology and Evolution 22: 2027-2039

Jones J, Nau K, Geraghty M, Erdmann R, Gould S (1999) Identification of Peroxisomal AcylCoA Thioesterases in Yeast and Humans. The Journal of Biological Chemistry 274: 9216-9223

Jump DB (2002) The Biochemistry of $n$-3 Polyunsatturated Fatty Acids. Journal of Biological Chemistry 277: 8755-8758

Kalscheuer R, Steinbüchel A (2003) A novel bifunctional wax ester synthase/acylCoA:diacylglycerol acyltransferase mediates wax ester and triacylglycerol biosynthesis in Acinetobacter calcoaceticus ADP1. J. Biol. Chem. 278: 8075-8082

Katavic V, Reed DW, Taylor DC, Giblin EM, Barton DL, Zou J, MacKenzie SL, Covello PS, Kunst L (1995) Alteration of seed fatty acid composition by an ethyl methanesulfonateinduced mutation in Arabidopsis thaliana affecting diacylglycerol acyltransferase activity. Plant Physiology 108: 399-409

Kaulmann U, Hertweck C (2002) Biosynthesis of polyunsaturated fatty acids by polyketide synthases. Angewandte Chemie International Edition 41: 1866-1869

Khozin-Goldberg I, Bigogno C, Shrestha P, Cohen Z (2002) Nitrogen starvation induces the accumulation of arachidonic acid in the freshwater green alga Parietochloris incisa (Trebuciophyceae). J. Phycol 38: 991-994 
Kim HU, Hsieh K, Ratnayake C, Huang AH (2002) A novel group of oleosins is present inside the pollen of Arabidopsis. J Biol Chem 277: 22677-22684

King A, Nam J-W, Han J, Hilliard J, Jaworski J (2007) Cuticular wax biosynthesis in petunia petals: cloning and characterization of an alcohol-acyltransferase that synthesizes waxesters. Planta 226: 381-394

Kinney A, Cahoon E, Damude H, Hitz W, Kolar C, Liu Z (2004) Production of very long chain polyunsaturated fatty acids in oilseed plants. In, Vol WO 2004/071467 A2

Knorzer K (1978) Evolution and spreading of Gold of Pleasure (Camelina sativa s.l.). Ber. Deutsch. Bot. Ges. 91: 187-195

Knutzon DS, Thurmond JM, Huang YS, Chaudhary S, Bobik EG, Jr., Chan GM, Kirchner SJ, Mukerji P (1998) Identification of $\Delta 5$-desaturase from Mortierella alpina by heterologous expression in Bakers' yeast and canola. J Biol Chem 273: 29360-29366

Korn ED (1964) The fatty acids of Euglena gracilis. Journal of Lipid Research 5: 352-362

Koziel MG, Carozzi NB, Desai N (1996) Optimizing expression of transgenes with an emphasis on post-transcriptional events. Plant Molecular Biology 32: 393-405

Kroon JT, Wei W, Simon WJ, Slabas AR (2006) Identification and functional expression of a type 2 acyl-CoA:diacylglycerol acyltransferase (DGAT2) in developing castor bean seeds which has high homology to the major triglyceride biosynthetic enzyme of fungi and animals. Phytochemistry 67: 2541-2549

Laemmli UK (1970) Cleavage of structural proteins during the assembly of the head of bacteriophage T4. Nature 227: $680-684$

Lands WEM (1958) Metabolism of glycerolipids: A comparison of lecithin and triglyceride synthesis. J. Biol. Chem. 231: 883-888

Lands WEM (2000) Stories about acyl chains. Biochimica et Biophysica Acta (BBA) - Molecular and Cell Biology of Lipids 1483: 1-14

Lang I (2007) New fatty acids, oxylipins and volatiles in microalgae. PhD thesis. Georg August University, Göttingen

Lardizabal K, Effertz R, Levering C, Mai J, Pedroso MC, Jury T, Aasen E, Gruys K, Bennett $\mathbf{K}$ (2008) Expression of Umbelopsis ramanniana DGAT2A in seed increases oil in soybean. Plant Physiology 148: 89-96

Lardizabal KD, Mai JT, Wagner NW, Wyrick A, Voelker T, Hawkins DJ (2001) Dgat2 is a new diacylglycerol acyltransferase gene family. purification, cloning, and expression in insect cells of two polypeptides from Mortierella ramanniana with diacylglycerol acyltransferase activity. J Biol Chem 276: 38862-38869

Larson TR, Graham IA (2001) Technical Advance: a novel technique for the sensitive quantification of acyl CoA esters from plant tissues. Plant J 25: 115-125.

Lassner MW, Levering CK, Davies HM, Knutzon DS (1995) Lysophosphatidic acid acyltransferase from meadow foam mediates insertion of erucic acid at the sn-2 position of triacylglycerol in transgenic rapeseed oil. Plant Physiology 109: 1389-1394

Lederer F (1994) The cytochrome b5-fold: an adaptable module. Biochimie 76: 674-692

Lee Y-K (2001) Microalgal mass culture systems and methods: Their limitation and potential. Journal of Applied Phycology 13: 307-315

Lehner R, Kuksis A (1996) Biosynthesis of triacylglycerols. Prog Lipid Res 35: 169-201

Lewis TE, Nichols PD, McMeekin TA (1999) The Biotechnological Potential of Thraustochytrids. Marine Biotechnology 1: 580-587

Li Y, Beisson F, Koo AJK, Molina I, Pollard M, Ohlrogge J (2007) Identification of acyltransferases required for cutin biosynthesis and production of cutin with suberin-like monomers. Proceedings of the National Academy of Sciences 104: 18339-18344

Liu L, Jiang X, Qin W, Yao G, Huan J (2007) Identification of the delta4 fatty acid desaturase gene from Thraustochytrium sp. FJN-10. Wei Sheng Wu Xue Tong Bao 34: 1154-1157 
Lu C, Kang J (2008) Generation of transgenic plants of a potential oilseed crop Camelina sativa by Agrobacterium-mediated transformation. Plant Cell Reports 27: 273-278

Lu C, Xin Z, Ren Z, Miquel M, Browse J (2009a) An enzyme regulating triacylglycerol composition is encoded by the ROD1 gene of Arabidopsis. Proceedings of the National Academy of Sciences 106: 18837-18842

Lu Y, Chi X, Yang Q, Li Z, Liu S, Gan Q, Qin S (2009b) Molecular cloning and stressdependent expression of a gene encoding $\Delta 12$-fatty acid desaturase in the Antarctic microalga Chlorella vulgaris NJ-7. Extremophiles 13: 875-884

Macdonald MH, Mogen BD, Hunt AG (1991) Characterization of the polyadenylation signal from the T-DNA-encoded octopine synthase gene. Nucleic Acids Research 19: 55755581

Man WC, Miyazaki M, Chu K, Ntambi J (2006a) Colocalization of SCD1 and DGAT2: implying preference for endogenous monounsaturated fatty acids in triglyceride synthesis. Journal of Lipid Research 47: 1928-1939

Man WC, Miyazaki M, Chu K, Ntambi JM (2006b) Membrane topology of mouse stearoyl-CoA desaturase 1. J. Biol. Chem. 281: 1251-1260

Mañas-Fernández A, Vilches-Ferrón M, Garrido-Cárdenas J, Belarbi EH, Alonso D, García-Maroto F (2009) Cloning and Molecular Characterization of the AcylCoA:Diacylglycerol Acyltransferase 1 (DGAT1) Gene from Echium. Lipids 44: 555-568

Metz J, Flatt J, Kuner J (2006) PUFA polyketide synthase systems and uses thereof. In MB Corporation, ed, Vol WO 2006/135866 A2

Metz JG, Kuner J, Rosenzweig B, Lippmeier JC, Roessler P, Zirkle R (2009) Biochemical characterization of polyunsaturated fatty acid synthesis in Schizochytrium: Release of the products as free fatty acids. Plant Physiology and Biochemistry 47: 472-478

Metz JG, Roessler P, Facciotti D, Levering C, Dittrich F, Lassner M, Valentine R, Lardizabal K, Domergue F, Yamada A, Yazawa K, Knauf V, Browse J (2001) Production of polyunsaturated fatty acids by polyketide synthases in both prokaryotes and eukaryotes. Science 293: 290-293

Meyer A, Cirpus P, Ott C, Schlecker R, Zahringer U, Heinz E (2003) Biosynthesis of docosahexaenoic acid in Euglena gracilis: biochemical and molecular evidence for the involvement of a $\Delta^{4}$-fatty acyl group desaturase. Biochemistry 42: 9779-9788

Meyer A, Kirsch H, Domergue F, Abbadi A, Sperling P, Bauer J, Cirpus P, Zank TK, Moreau H, Roscoe TJ, Zahringer U, Heinz E (2004) Novel fatty acid elongases and their use for the reconstitution of docosahexaenoic acid biosynthesis. J. Lipid Res. 45: 1899-1909

Meyer TS, Lamberts BL (1965) Use of coomassie brilliant blue R250 for the electrophoresis of microgram quantities of parotid saliva proteins on acrylamide-gel strips. Biochimica et Biophysica Acta (BBA) - General Subjects 107: 144-145

Mhaske V, Beldjilali K, Ohlrogge J, Pollard M (2005) Isolation and characterization of an Arabidopsis thaliana knockout line for phospholipid:diacylglycerol transacylase gene (At5g13640). Plant Physiology and Biochemistry 43: 413-417

Michaelson LV, Lazarus CM, Griffiths G, Napier JA, Stobart AK (1998) Isolation of a $\Delta^{5}$-fatty acid desaturase gene from Mortierella alpina. J Biol Chem 273: 19055-19059

Milcamps A, Tumaney AW, Paddock T, Pan DA, Ohlrogge J, Pollard M (2005) Isolation of a Gene Encoding a 1,2-Diacylglycerol-sn-acetyl-CoA Acetyltransferase from Developing Seeds of Euonymus alatus. J. Biol. Chem. 280: 5370-5377

Millar AA, Kunst L (1997) Very-long-chain fatty acid biosynthesis is controlled through the expression and specificity of the condensing enzyme. Plant Journal 12: 121-131

Millar AA, Smith MA, Kunst L (2000) All fatty acids are not equal: discrimination in plant membrane lipids. Trends Plant Sci 5: 95-101

Miquel M, Browse J (1992) Arabidopsis mutants deficient in polyunsaturated fatty acid synthesis. Journal of Biological Chemistry 267: 1502-1509 
Mitchell AG, Martin CE (1997) Fah1p, a Saccharomyces cerevisiae cytochrome $b 5$ fusion protein, and its Arabidopsis thaliana homolog that lacks the cytochrome $b 5$ domain both function in the $\alpha$-hydroxylation of sphingolipid-associated very long chain fatty acids. J. Biol. Chem. 272: 28281-28288

Mudd JB, Stumpf PK (1961) Fat Metabolism in Higher Plants. XIV. Factors affecting the synthesis of oleic acid by particulate preparations from avocado mesocarp. J. Biol. Chem. 236: $2602-2609$

Müller KD, Husmann H, Nalik H, Schomburg G (1990) Trans-esterification of fatty acids from microorganisms and human blood serum by trimethylsulfonium hydroxide (TMSH) for GC analysis. Chromatographia 30: 245-248

Mullis KB, Faloona FA (1987) Specific synthesis of DNA in vitro via a polymerase-catalyzed chain reaction. Methods Enzymology 155: 335-350

Murphy DJ, Vance J (1999) Mechanisms of lipid body formation. Trends in Biochemical Sciences 24: 109-115

Nagai J, Bloch K (1966) Enzymatic desaturation of stearyl acyl carrier protein. J. Biol. Chem. 241: $1925-1927$

Nagai J, Bloch K (1968) Enzymatic desaturation of stearyl acyl carrier protein. J. Biol. Chem. 243: 4626-4633

Nakamura MT, Nara TY (2003) Essential fatty acid synthesis and its regulation in mammals. Prostaglandins, Leukotrienes and Essential Fatty Acids 68: 145-150

Nakanishi H, Shindou H, Hishikawa D, Harayama T, Ogasawara R, Suwabe A, Taguchi R, Shimizu T (2006) Cloning and Characterization of Mouse Lung-type AcylCoA:Lysophosphatidylcholine Acyltransferase 1 (LPCAT1): Expression in alveolar type II cells and possible involvement in surfactant production. J. Biol. Chem. 281: 2014020147

Napier JA (2007a) The Production of Unusual Fatty Acids in Transgenic Plants. Annual Review of Plant Biology 58: 295-319

Napier JA (2007b) Transgenic plants as a source of fish oils: healthy, sustainable and GM. Journal of the Science of Food and Agriculture 87: 8-12

Napier JA, Hey SJ, Lacey DJ, Shewry PR (1998) Identification of a Caenorhabditis elegans Delta6-fatty-acid-desaturase by heterologous expression in Saccharomyces cerevisiae. Biochem J 330 (Pt 2): 611-614

Napier JA, Michaelson LV, Sayanova O (2003) The role of cytochrome b5 fusion desaturases in the synthesis of polyunsaturated fatty acids. Prostaglandins, Leukotrienes and Essential Fatty Acids 68: 135-143

Napier JA, Sayanova O, Stobart AK, Shewry PR (1997) A new class of cytochrome b5 fusion proteins. Biochem J 328: 717-718

Naylor R, Goldburg RJ, Primavera JH, Kautsky N, Beveridge MC, Clay J, Folke C, Lubchenco J, Mooney H, M T (2000) Effect of aquaculture on world fish supplies. Nature 29: 1017-1024

Norman HA, Pillai P, St. John JB (1991) In vitro desaturation of monogalactosyldiacylglycerol and phosphatidylcholine molecular species by chloroplast homogenates. Phytochemistry 30: 2217-2222

Ntamack AG, Karpichev IV, Gould SJ, Small GM, Schulz H (2009) Oleate [beta]-oxidation in yeast involves thioesterase but not Yor180c protein that is not a dienoyl-CoA isomerase. Biochimica et Biophysica Acta (BBA) - Molecular and Cell Biology of Lipids 1791: 371-378

Nykiforuk CL, Furukawa-Stoffer TL, Huff PW, Sarna M, Laroche A, Moloney MM, Weselake RJ (2002) Characterization of cDNAs encoding diacylglycerol acyltransferase from cultures of Brassica napus and sucrose-mediated induction of enzyme biosynthesis. Biochim Biophys Acta 1580: 95-109 
O’Quin J, Mullen R, Dyer J (2009) Addition of an N-terminal epitope tag significantly increases the activity of plant fatty acid desaturases expressed in yeast cells. Applied Microbiology and Biotechnology 83: 117-125

Oelkers P, Cromley D, Padamsee M, Billheimer JT, Sturley SL (2002) The DGA1 gene determines a second triglyceride synthetic pathway in yeast. The Journal of Biological Chemistry 277: 8877-8881

Oelkers P, Tinkelenberg A, Erdeniz N, Cromley D, Billheimer JT, Sturley SL (2000) A lecithin cholesterol acyltransferase-like gene mediates diacylglycerol esterification in yeast. J. Biol. Chem. 275: 15609-15612

Ohlrogge JB, Browse J (1995) Lipid Biosynthesis. The Plant Cell 7: 957-970

Ohlrogge JB, Jaworski JG (1997) Regulation of fatty acid synthesis. Annual Review of Plant Physiology and Plant Molecular Biology 48: 109-136

Okayasu T, Nagao M, Ishibashi T, Imai Y (1981) Purification and partial characterization of linoleoyl-CoA desaturase from rat liver microsomes. Arch Biochem Biophys 206: 21-28

Okuley J, Lightner J, Feldmann K, Yadav N, Lark E, Browse J (1994) Arabidopsis FAD2 Gene Encodes the Enzyme That Is Essential for Polyunsaturated Lipid Synthesis. Plant Cell 6: 147-158

Oshino N, Imai Y, Sato R (1966) Electron-transfer mechanism associated with fatty acid desaturation catalyzed. Biochimica et Biophysica Acta 128: 13-28

Palenik B, Grimwood J, Aerts A, Rouze P, Salamov A, Putnam N, Dupont C, Jorgensen R, Derelle E, Rombauts S, Zhou K, Otillar R, Merchant SS, Podell S, Gaasterland T, Napoli C, Gendler K, Manuell A, Tai V, Vallon O, Piganeau G, Jancek S, Heijde M, Jabbari K, Bowler C, Lohr M, Robbens S, Werner G, Dubchak I, Pazour GJ, Ren Q, Paulsen I, Delwiche C, Schmutz J, Rokhsar D, Van de Peer Y, Moreau H, Grigoriev IV (2007) The tiny eukaryote Ostreococcus provides genomic insights into the paradox of plankton speciation. PNAS 104: 7705-7710

Parker-Barnes JM, Das T, Bobik E, Leonard AE, Thurmond JM, Chaung LT, Huang YS, Mukerji P (2000) Identification and characterization of an enzyme involved in the elongation of n-6 and n-3 polyunsaturated fatty acids. Proc Natl Acad Sci U S A 97: 82848289

Pauly D, Christensen V, Guenette S, Pitcher TJ, Sumaila UR, Walters CJ, Watson R, Zeller D (2002) Towards sustainability in world fisheries. Nature 418: 689-695

Pawlosky RJ, Hibbeln JR, Novotny JA, Salem N, Jr. (2001) Physiological compartmental analysis of alpha-linolenic acid metabolism in adult humans. J Lipid Res 42: 1257-1265

Pereira SL, Huang YS, Bobik EG, Kinney AJ, Stecca KL, Packer JC, Mukerji P (2004a) A novel omega3-fatty acid desaturase involved in the biosynthesis of eicosapentaenoic acid. Biochem J 378: 665-671

Pereira SL, Leonard AE, Huang YS, Chuang LT, Mukerji P (2004b) Identification of two novel microalgal enzymes involved in the conversion of the omega3-fatty acid, eicosapentaenoic acid, into docosahexaenoic acid. Biochem J 384: 357-366

Prasad MR, Sreekrishna K, Joshi VC (1980) Topology of the delta 9 terminal desaturase in chicken liver microsomes and artificial micelles. Inhibition of the enzyme activity by the antibody and susceptibility of the enzyme to proteolysis. J Biol Chem 255: 2583-2589

Qi B, Beaudoin F, Fraser T, Stobart AK, Napier JA, Lazarus CM (2002) Identification of a cDNA encoding a novel C18- $\Delta^{9}$ polyunsaturated fatty acid-specific elongating activity from the docosahexaenoic acid (DHA)-producing microalga, Isochrysis galbana. FEBS Lett 510: 159-165

Qi B, Fraser T, Mugford S, Dobson G, Sayanova O, Butler J, Napier JA, Stobart AK, Lazarus CM (2004) Production of very long chain polyunsaturated omega-3 and omega-6 fatty acids in plants. Nat Biotechnol 22: 739-745 
Qiu X, Hong Ha, MacKenzie SL (2001) Identification of a $\Delta 4$ Fatty Acid Desaturase from Thraustochytrium sp. Involved in the Biosynthesis of Docosahexanoic Acid by Heterologous Expression in Saccharomyces cerevisiae and Brassica juncea. The Journal of Biological Chemistry 276: 31561-31566

Rice P, Longden I, Bleasby A (2000) EMBOSS: the european molecular biology open software suite. Trends Genet 16: 276-277

Riekhof WR, Wu J, Jones JL, Voelker DR (2007) Identification and Characterization of the Major Lysophosphatidylethanolamine Acyltransferase in Saccharomyces cerevisiae. J. Biol. Chem. 282: 28344-28352

Robert SS, Singh PS, Zhou X-R, Petrie JR, Blackburn SI, Mansour PM, Nichols PD, Liu Q, Green AG (2005) Metabolic engineering of Arabidopsis to produce nutritionally important DHA in seed oil. Functional Plant Biology 32: 473-479

Rosendal J, Knudsen J (1992) A fast and versatile method for extraction and quantitation of long-chain acyl-CoA esters from tissue: content of individual long-chain acyl-CoA esters in various tissues from fed rat. Anal Biochem 207: 63-67

Roughan PG, Slack CR (1982) Cellular organization of glycerolipid metabolism. Annu. Rev. Plant Physiol. 33: 97-132

Routaboul JM, Benning C, Bechtold N, Caboche M, Lepiniec L (1999) The TAG1 locus of Arabidopsis encodes for a diacylglycerol acyltransferase. Plant Physiology and Biochemistry 37: 831-840

Saha S, Enugutti B, Rajakumari S, Rajasekharan R (2006) Cytosolic triacylglycerol biosynthetic pathway in oilseeds: Molecular cloning and expression of peanut cytosolic diacylglycerol acyltransferase. Plant Physiol.: pp.106.082198

Sandager L, Gustavsson MH, Stahl U, Dahlqvist A, Wiberg E, Banas A, Lenman M, Ronne H, Stymne S (2002) Storage lipid synthesis is non-essential in yeast. J Biol Chem 277: 6478-6482

Sanger F, Nicklen S, Coulson AR (1977) DNA sequencing with chain-terminating inhibitors. Proc. Natl. Acad. Sci. USA 74: 5463-5467

Sayanova O, Beaudoin F, Libisch B, Castel A, Shewry PR, Napier JA (2001) Mutagenesis and heterologous expression in yeast of a plant $\Delta^{6}$ - fatty acid desaturase. J Exp Bot 52: 1581-1585.

Sayanova O, Haslam R, Guschina I, Lloyd D, Christie WW, Harwood JL, Napier JA (2006) A bifunctional $\Delta 12, \Delta 15$-desaturase from Acanthamoeba castellanii directs the synthesis of highly unusual n-1 series unsaturated fatty acids. J. Biol. Chem. 281: 36533-36541

Sayanova O, Smith MA, Lapinskas P, Stobart AK, Dobson G, Christie WW, Shewry PR, Napier JA (1997) Expression of a borage desaturase cDNA containing an $\mathrm{N}$-terminal cytochrome b5 domain results in the accumulation of high levels of $\Delta 6$-desaturated fatty acids in transgenic tobacco. Proc Natl Acad Sci U S A 94: 4211-4216

Sayanova OV, Napier JA (2004) Eicosapentaenoic acid: biosynthetic routes and the potential for synthesis in transgenic plants. Phytochemistry 65: 147-158

Schmidt H, Heinz E (1990) Desaturation of Oleoyl Groups in Envelope Membranes from Spinach Chloroplasts. Proceedings of the National Academy of Sciences of the United States of America 87: 9477-9480

Schwarzenbach AM (1971) Observations on spherosomal membranes. Cytobiologie 4: 415417

Shanklin J, Cahoon EB (1998) Desaturation and related modifications of fatty acids. Annual Review of Plant Physiology and Plant Molecular Biology 49: 611-641

Shanklin J, Mullins C, Somerville C (1991) Sequence of a complementary DNA from Cucumis-Sativus $L$ encoding the stearoyl-acyl-carrier protein desaturase. Plant Physiology 97: $467-468$ 
Shanklin J, Whittle E, Fox BG (1994) Eight histidine residues are catalytically essential in a membrane-associated iron enzyme, stearoyl-CoA desaturase, and are conserved in alkane hydroxylase and xylene monooxygenase. Biochemistry 33: 12787-12794

Shimizu T, Ohto T, Kita Y (2006) Cytosolic phospholipase A2: Biochemical properties and physiological roles. In, Vol 58. Informa Healthcare, pp 328-333

Shimojima M, Ohta H, Iwamatsu A, Masuda T, Shioi Y, Takamiya K-i (1997) Cloning of the gene for monogalactosyldiacylglycerol synthase and its evolutionary origin. Proceedings of the National Academy of Sciences of the United States of America 94: 333-337

Shockey JM, Gidda SK, Chapital DC, Kuan J-C, Dhanoa PK, Bland JM, Rothstein SJ, Mullen RT, Dyer JM (2006) Tung tree DGAT1 and DGAT2 have nonredundant functions in triacylglycerol biosynthesis and are localized to different subdomains of the endoplasmic reticulum. Plant Cell 18: 2294-2313

Silhavy TJ, Berman ML, Enquist LW, Cold Spring Harbor L (1984) Experiments with gene fusions / Thomas J. Silhavy, Michael L. Berman, Lynn W. Enquist. In. New York : Cold Spring Harbor Laboratory

Simopoulos AP (2002) Omega-3 Fatty Acids in Inflammation and Autoimmune Diseases. J Am Coll Nutr 21: 495-505

Simopoulos AP (2006) Evolutionary aspects of diet, the omega-6/omega-3 ratio and genetic variation: nutritional implications for chronic diseases. Biomed Pharmacother 60: 502507

Simpson GG, Filipowicz W (1996) Splicing of precursors to mRNA in higher plants: mechanism, regulation and sub-nuclear organisation of the spliceosomal machinery. Plant Molecular Biology 32: 1-41

Somerville C, Browse J (1991) Plant Lipids - Metabolism, Mutants, and Membranes. Science 252: $80-87$

Sorger D, Daum G (2002) Synthesis of triacylglycerols by the acyl-coenzyme A:diacyl-glycerol acyltransferase Dga1p in lipid particles of the yeast Saccharomyces cerevisiae. Journal of Bacteriology 184: 519-524

Spatz L, Strittmatter P (1971) A form of cytochrome b5 that contains an additional hydrophobic sequence of 40 amino acid residues. PNAS 68: 1042-1046

Sperling P, Heinz E (2001) Desaturases fused to their electron donor. Eur. J. Lipid Sci. Technol. 103: 158-180

Sperling P, Schmidt H, Heinz E (1995) A cytochrome-b5-containing fusion protein similar to plant acyl lipid desaturases. Eur J Biochem 232: 798-805

Sperling P, Ternes P, Zank TK, Heinz E (2003) The evolution of desaturases. Prostaglandins Leukot. Essent. Fatty Acids 68: 73-95

Sprecher H (2000) Metabolism of highly unsaturated n-3 and n-6 fatty acids. Biochimica et Biophysica Acta (BBA) - Molecular and Cell Biology of Lipids 1486: 219-231

Sprecher H, Chen Q, Yin FQ (1999) Regulation of the biosynthesis of 22:5n-6 and 22:6n-3: A complex intracellular process. Lipids 34: 153-156

Sprecher H, Luthria D, Mohammed B, Baykousheva S (1995) Reevaluation of the pathways for the biosynthesis of polyunsaturated fatty acids. J. Lipid Res. 36: 2471-2477

Stahl U, Carlsson AS, Lenman M, Dahlqvist A, Huang B, Banas W, Banas A, Stymne S (2004) Cloning and functional characterization of a phospholipid:diacylglycerol acyltransferase from Arabidopsis. Plant Physiol. 135: 1324-1335

Stahl U, Stalberg K, Stymne S, Ronne H (2008) A family of eukaryotic lysophospholipid acyltransferases with broad specificity. FEBS Lett 582: 305-309

Stalberg K, StahI U, Stymne S, Ohlrogge J (2009) Characterization of two Arabidopsis thaliana acyltransferases with preference for lysophosphatidylethanolamine. BMC Plant Biology 9: 60 
Stobart AK, Stymne S, Höglund S (1986) Safflower microsomes catalyse oil accumulation in vitro: a model system. Planta 169: 33-37

Stobart K, Mancha M, Lenman M, Dahlqvist A, Stymne S (1997) Triacylglycerols are synthesised and utilized by transacylation reactions in microsomal preparations from developing safflower (Carthamus tinctorius L.) seeds. Planta 203: 58-66

Stoveken T, Kalscheuer R, Malkus U, Reichelt R, Steinbuchel A (2005) The Wax Ester Synthase/Acyl Coenzyme A:Diacylglycerol Acyltransferase from Acinetobacter sp. Strain ADP1: Characterization of a Novel Type of Acyltransferase. J. Bacteriol. 187: 13691376

Strittmatter P, Spatz L, Corcoran D, Rogers MJ, Setlow B, Redline R (1974) Purification and properties of rat liver microsomal stearyl coenzyme A desaturase. PNAS 71: 4565-4569

Stukey JE, McDonough VM, Martin CE (1990) The OLE1 gene of Saccharomyces cerevisiae encodes the $\Delta 9$ fatty acid desaturase and can be functionally replaced by the rat stearoyl-CoA desaturase gene. Journal of Biological Chemistry 265: 20144-20149

Stymne S, Stobart AK (1987) Triacylglycerol biosynthesis. The Biochemistry of plants: a comprehensive treatise 9: 175-214

Stymne S, Stobart AK (1984) Evidence for the reversibility of the acylCoA:lysophosphatidylcholine acyltransferase in microsomal preparations from developing safflower (Carthamus tinctorius L.) cotyledons and rat liver. Biochem J 223: 305-314

Stymne S, Stobart AK (1985) Involvement of acyl exchange between acyl-CoA and phosphatidylcholine in the remodelling of phosphatidylcholine in microsomal preparations of rat lung. Biochim Biophys Acta 837: 239-250.

Stymne S, Stobart AK (1986) Biosynthesis of gamma-linolenic acid in cotyledons and microsomal preparations of the developing seeds of common borage (Borago officinalis). Biochem. J. 240: 385-393

Suggs SV, Hirose T, Miyake EH, Kawashima MJ, Johnson KI, Wallace RB, eds (1981) Using purified genes, Vol 23. Acad. Press, New York

Tamaki H, Shimada A, Ito Y, Ohya M, Takase J, Miyashita M, Miyagawa H, Nozaki H, Nakayama R, Kumagai H (2007) LPT1 Encodes a Membrane-bound O-Acyltransferase Involved in the Acylation of Lysophospholipids in the Yeast Saccharomyces cerevisiae. J. Biol. Chem. 282: 34288-34298

Taylor DC, Zhang Y, Kumar A, Francis T, Giblin EM, Barton DL, Ferrie JR, Laroche A, Shah S, Zhu W, Snyder CL, Hall L, Rakow G, Harwood JL, Weselake RJ (2009) Molecular modification of triacylglycerol accumulation by over-expression of DGAT1 to produce canola with increased seed oil content under field conditions. Botany 87: 533543

Thelen JJ, Ohlrogge JB (2002) Metabolic Engineering of Fatty Acid Biosynthesis in Plants. Metabolic Engineering 4: 12-21

Thompson GA, Scherer DE, Foxall-Van Aken S, Kenny JW, Young HL, Shintani DK, Kridl JC, Knauf VC (1991) Primary structures of the precursor and mature forms of stearoylacyl carrier protein desaturase from safflower embryos and requirement of ferredoxin for enzyme activity. Proceedings of the National Academy of Sciences of the United States of America 88: 2578-2582

Thompson J, Gibson T, Plewniak F, Jeanmougin F, Higgins D (1997) The CLUSTAL X windows interface: flexible strategies for multiple sequence alignment aided by quality tools. Nucleic Acids Res 25: 4876- 4882

Tonon T, Harvey D, Larson TR, Graham IA (2003) Identification of a very long chain polyunsaturated fatty acid $\Delta 4$-desaturase from the microalga Pavlova lutheri. FEBS Lett 553: 440-444

Tonon T, Qing R, Harvey D, Li Y, Larson TR, Graham IA (2005a) Identification of a LongChain Polyunsaturated Fatty Acid Acyl-Coenzyme A Synthetase from the Diatom Thalassiosira pseudonana. Plant Physiol. 138: 402-408 
Tonon T, Sayanova O, Michaelson LV, Qing R, Harvey D, Larson TR, Li Y, Napier JA, Graham IA (2005b) Fatty acid desaturases from the microalga Thalassiosira pseudonana. FEBS Journal 272: 3401-3412

Trooskens G, De Beule D, Decouttere F, Van Criekinge W (2005) Phylogenetic trees: visualizing, customizing and detecting incongruence. Bioinformatics 21: 3801-3802

Truksa M, Vrinten P, Qiu X (2009) Metabolic engineering of plants for polyunsaturated fatty acid production. Molecular Breeding 23: 1-11

Tumaney AW, Shekar S, Rajasekharan R (2001) Identification, Purification, and Characterization of Monoacylglycerol Acyltransferase from Developing Peanut Cotyledons. The Journal of Biological Chemistry 276: 10847-10852

Valachovič M, Hronská L, Hapala I (2001) Anaerobiosis induces complex changes in sterol esterification pattern in the yeast Saccharomyces cerevisiae. In, Vol 197. Blackwell Publishing Ltd, pp 41-45

Venegas-Calerón M, Sayanova O, Napier JA (2010) An alternative to fish oils: Metabolic engineering of oil-seed crops to produce omega-3 long chain polyunsaturated fatty acids. Progress in Lipid Research 49: 108-119

Vicient CM, Delseny M (1999) Isolation of Total RNA from Arabidopsis thaliana Seeds. Analytical Biochemistry 268: 412-413

Vijayan P, Browse J (2002) Photoinhibition in mutants of Arabidopsis deficient in thylakoid unsaturation. Plant Physiol 129: 876-885

Voss A, Reinhart M, Sankarappa S, Sprecher H (1991) The metabolism of 7,10,13,16,19docosapentaenoic acid to 4,7,10,13,16,19-docosahexaenoic acid in rat liver is independent of a delta-4-desaturase. J. Biol. Chem. 266: 19995-20000

Wach A, Brachat A, PohImann R, Philippsen P (1994) New heterologous modules for classical or PCR-based gene disruptions in Saccharomyces cerevisiae. Yeast 10: 1793-1808

Wada H, Schmidt H, Heinz E, Murata N (1993) In vitro ferredoxin-dependent desaturation of fatty acids in cyanobacterial thylakoid membranes. J. Bacteriol. 175: 544-547

Wagner M (2008) Identifizierung und funktionale Charakterisierung neuartiger Acyltransferasen aus Mikroalgen Georg August University, Goettingen

Wagner M, Hoppe K, Czabany T, Heilmann M, Daum G, Feussner I, Fulda M (2010) Identification and characterization of an acyl-CoA:diacylglycerol acyltransferase 2 (DGAT2) gene from the microalga O. tauri. Plant Physiology and Biochemistry 48: 407-416

Wallis JG, Browse $\mathbf{J}(1999)$ The $\Delta^{8}$-desaturase of Euglena gracilis: An alternate pathway for synthesis of 20-carbon polyunsaturated fatty acids. Archives of Biochemistry and Biophysics 365: 307-316

Wang H-W, Zhang J-S, Gai J-Y, Chen S-Y (2006) Cloning and comparative analysis of the gene encoding diacylglycerol acyltransferase from wild type and cultivated soybean. TAG Theoretical and Applied Genetics 112: 1086-1097

Weselake RJ, Shah S, Tang M, Quant PA, Snyder CL, Furukawa-Stoffer TL, Zhu W, Taylor DC, Zou J, Kumar A, Hall L, Laroche A, Rakow G, Raney P, Moloney MM, Harwood JL (2008) Metabolic control analysis is helpful for informed genetic manipulation of oilseed rape (Brassica napus) to increase seed oil content. J Exp Bot 59: 3543-3549

Whitney HM, Michaelson LV, Sayanova O, Pickett JA, Napier JA (2003) Functional characterisation of two cytochrome $b_{5}$-fusion desaturases from Anemone leveillei: the unexpected identification of a fatty acid $\Delta^{6}$-desaturase. Planta 217: 983-992

Wu G, Truksa M, Datla N, Vrinten P, Bauer J, Zank T, Cirpus P, Heinz E, Qiu X (2005) Stepwise engineering to produce high yields of very long-chain polyunsaturated fatty acids in plants. Nat Biotech 23: 1013-1017 
Xu J, Francis T, Mietkiewska E, Giblin EM, Barton DL, Zhang Y, Zhang M, Taylor DC (2008) Cloning and characterization of an acyl-CoA-dependent diacylglycerol acyltransferase 1 (DGAT1) gene from Tropaeolum majus, and a study of the functional motifs of the DGAT protein using site-directed mutagenesis to modify enzyme activity and oil content. Plant Biotechnology Journal 6: 799-818

Yang H, Bard M, Bruner DA, Gleeson A, Deckelbaum RJ, Aljinovic G, Pohl TM, Rothstein R, Sturley SL (1996) Sterol Esterification in Yeast: A Two-Gene Process. Science 272: $1353-1356$

Yu C, Kennedy N, Chang C, Rothblatt J (1996) Molecular Cloning and Characterization of Two Isoforms of Saccharomyces cerevisiae Acyl-CoA:Sterol Acyltransferase. The Journal of Biological Chemistry 271: 24157-24163

Zank TK, Zahringer U, Beckmann C, Pohnert G, Boland W, Holtorf H, Reski R, Lerchl J, Heinz E (2002) Cloning and functional characterisation of an enzyme involved in the elongation of $\Delta 6$-polyunsaturated fatty acids from the moss Physcomitrella patens. Plant J 31: 255-268

Zhang M, Fan J, Taylor DC, Ohlrogge JB (2009) DGAT1 and PDAT1 Acyltransferases Have Overlapping Functions in Arabidopsis Triacylglycerol Biosynthesis and Are Essential for Normal Pollen and Seed Development. The Plant Cell 21: 3885-3901

Zheng P, Allen WB, Roesler K, Williams ME, Zhang S, Li J, Glassman K, Ranch J, Nubel D, Solawetz W, Bhattramakki D, Llaca V, Deschamps S, Zhong G-Y, Tarczynski MC, Shen B (2008) A phenylalanine in DGAT is a key determinant of oil content and composition in maize. Nat Genet 40: 367-372

Zhou X-R, Robert SS, Petrie JR, Frampton DMF, Mansour MP, Blackburn SI, Nichols PD, Green AG, Singh SP (2007) Isolation and characterization of genes from the marine microalga Pavlova salina encoding three front-end desaturases involved in docosahexaenoic acid biosynthesis. Phytochemistry 68: 785-796

Zou J, Katavic V, Giblin EM, Barton DL, MacKenzie SL, Keller WA, Hu X, Taylor DC (1997) Modification of Seed Oil Content and Acyl Composition in the Brassicaceae by Expression of a Yeast sn-2 Acyltransferase Gene. Plant Cell 9: 909-923

Zou J, Wei Y, Jako C, Kumar A, Selvaraj G, Taylor DC (1999) The Arabidopsis thaliana TAG1 mutant has a mutation in a diacylglycerol acyltransferase gene. Plant Journal 19: 645-653

Zweytick D, Athenstaedt K, Daum G (2000) Intracellular lipid particles of eukaryotic cells. Biochim Biophys Acta 1469: 101-120 
- 158 - 


\section{Appendix}

The following tables give the sequences of primers used for isolation and cloning of genes. Primer sequences are given in the $5^{\prime}$ to $3^{\prime}$ orientation and restriction sites for cloning into expression plasmids are in bold.

Appendix 1. OILPCAT and OtLPCAT

\begin{tabular}{|ll|}
\hline \multicolumn{1}{|c|}{ Name } & \multicolumn{1}{c|}{ Sequence } \\
\hline OlLPCATcoforBamHI & ATGCGGATCCACATAATGTTATCTGCCGCCGCATTTGG \\
\hline OlLPCATcorevApal & ATGCGGGCCTTAGGCAGACTTAGGTTTACTTGACC \\
\hline OlLPCATcorevNotI & GCATGCGGCCGCTTAGGCAGACTTAGGTTTACTTGACC \\
\hline OlLPCATcoforHindIII & CCGGATGCAAGCTTATGTTATCTGCCGCCGCATTTGG \\
\hline OILPCATcorevXhol & CCGGATGCCTCGAGTTAGGCAGACTTAGGTTTACTTGACC \\
\hline OtLPCATtcoforBamHI & CCGGATGCGGATCCATGGACGCTGCTTTGGACGC \\
\hline OtLPCATrevNhel & CCGGATGCGCTAGCTTAAGCCGTCTTGGGTTTGCTCG \\
\hline OtLPCATtcorevNotI & CCGGATGCGCGGCCGCTTAAGCCGTCTTGGGTTTGCTCG \\
\hline OtLPCATtcorev-StopXbal & CCGGATGCTCTAGAAGCCGTCTTGGGTTTGCTCG \\
\hline OtLPCATtcoforSpel & CCGGATGCACTAGTATGGACGCTGCTTTGGACGC \\
\hline OtLPCATtcorevHindIII & CCGGATGCAAGCTTTTAAGCCGTCTTGGGTTTGCTCG \\
\hline
\end{tabular}

Appendix 2. OtDGAT2A, OtDGAT2B and OtDGAT2C

\begin{tabular}{|ll|}
\hline \multicolumn{1}{|c|}{ Name } & \multicolumn{1}{c|}{ Sequence } \\
\hline OtDGAT2AcoforEcoRI & ATGCGAATTCACATAATGTCCAGACCAGAACCAGGAAGTC \\
\hline OtDGAT2AcorevNotI & GCATGCGGCCGCTTATACCAATTTAGTTCTCTGAGTTGG \\
\hline OtDGAT2BtcoforBamHI & ATGCGGATCCACATAATGGGTTCCAGATCTATTGTTGACC \\
\hline OtDGAT2BtcorevNotI & GCATGCGGCCGCTCAGCACACGACGAGGTCGACG \\
\hline OtDGAT2CcoforEcoRI & ATGCGAATTCACATAATGATTTATGCATTTTTGTTGTCAGC \\
\hline OtDGAT2CcorevNotI & GCATGCGGCCGCCTACTTAAATGTCATAGCTTGC \\
\hline
\end{tabular}


Appendix 3. OtPDAT

\begin{tabular}{|lc|}
\hline \multicolumn{1}{|c|}{ Name } & Sequence \\
\hline OtPDATtcoforEcoRI & ATGCGAATTCACATAATGGCTGTTACTAGAAGAACTACTAG \\
\hline OtPDATtcorevNotI & GCATGCGGCCGCCTAAAACGACTCGCGTGGGTTGG \\
\hline
\end{tabular}

Appendix 4. Old4

\begin{tabular}{|ll|}
\hline \multicolumn{1}{|c|}{ Name } & \multicolumn{1}{c|}{ Sequence } \\
\hline Old4for & ATGCCCTCCGCCGCGCGCTCCCGAG \\
\hline Old4hpggfor & CATCGCCATCCCGGCGGCGCGCAATTG \\
\hline Old4mfor & GAGTGGCGGTATCATCACATGGTGAG \\
\hline Old4hpggrev & CAATTGCGCGCCGCCGGGATGGCGATG \\
\hline Old4mrev & CTCACCATGTGATGATACCGCCACTC \\
\hline Old4rev & CTACATGGCCTTCGGTCGTTGATGG \\
\hline Old4revNotl & GCATGCGGCCGCCTACATGGCCTTCGGTCGTTGATGG \\
\hline Old4tcofor & ATGCCATCTGCTGCTAGATCTAGAGCTTCTAAAAGAGCTAA \\
\hline Old4tcoforEcoRI & CGACACTACTGATGTTGCTACGACCGCTCCCGAGG- \\
\hline Old4revXhol & CCGGATGCGAATTCATGCCATCTGCTGCTAGATCTAG \\
\hline
\end{tabular}

Appendix 5. Egd4

\begin{tabular}{|ll|}
\hline \multicolumn{1}{|c|}{ Name } & \multicolumn{1}{c|}{ Sequence } \\
\hline Egd4forBamHI & $\begin{array}{l}\text { AGGATCCAGATCTCACCATGTTGGTGCTGTTTGGCAATT- } \\
\text { TCTATG }\end{array}$ \\
\hline Egd4revXhol & ACTCGAGCCCGGGTTATGACTTTTTGTCCCCGTTCGTTG \\
\hline Egd4forEcoRl & CCGGATGCGAATTCATGTTGGTGCTGTTTGGCAATTTC \\
\hline
\end{tabular}

Appendix 6. TpLACS

\begin{tabular}{|ll|}
\hline \multicolumn{1}{|c|}{ Name } & \multicolumn{1}{c|}{ Sequence } \\
\hline TpLACSfor & ATGACGAACAAATGGTCCATAGAAG \\
\hline TpLACSrev & TTACAACTTGCTCTGAGGAGAATC \\
\hline TpLACSforApal & ATGCGGGCCCACATAATGACGAACAAATGGTCCATAGAAG \\
\hline TpLACSrevNhel & GCATGCTAGCTTACAACTTGCTCTGAGGAGAATC \\
\hline
\end{tabular}


Appendix 7. CsMGDGS

\begin{tabular}{|cl|}
\hline \multicolumn{1}{|c|}{ Name } & \multicolumn{1}{c|}{ Sequence } \\
\hline CsMGDGSforBamHI & $\begin{array}{l}\text { CCGGATGCGGATCCACATAATGGGTGTTTCAGATGAAAC- } \\
\text { CAATGG }\end{array}$ \\
\hline CsMGDGSrevNhel & $\begin{array}{l}\text { CCGGATGCGCTAGCTCAGCCGGAATATTGTGGTACAA- } \\
\text { AAC }\end{array}$ \\
\hline
\end{tabular}

Appendix 8. AtDGAT1 and AtDGAT2

\begin{tabular}{|ll|}
\hline \multicolumn{1}{|c|}{ Name } & \multicolumn{1}{c|}{ Sequence } \\
\hline AtDGAT1forkpnl & CCGGATGCGGTACCATGGCGATTTTGGATTCTGCTGG \\
\hline AtDGAT1revXhol & CCGGATGCCTCGAGTCATGACATCGATCCTTTTCGG \\
\hline AtDGAT2forEcoRl & CCGGATGCGAATTCATGGGTGGTTCCAGAGAGTTCCG \\
\hline AtDGAT2revXhol & CCGGATGCCTCGAGTCAAAGAATTTTCAGCTCAAG \\
\hline
\end{tabular}




\section{Curriculum Vitae}

$\begin{array}{ll}\text { Name } & \text { Katharina Ahmann (née Hoppe) } \\ \text { Date of birth } & \text { May 25, 1984 } \\ \text { Place of birth } & \text { Osnabrück, Germany } \\ \text { Family status } & \text { married }\end{array}$

\section{Education and Qualifications}

since $10 / 2007$

PhD program Molecular Biology

International Max Planck Research School

PhD thesis with Prof. Dr. Ivo Feußner at the Albrecht-von-

Haller-Institute of Plant Sciences, Georg August Univer-

sity Göttingen

$10 / 2006-03 / 2008$

\section{MSc program Molecular Biology}

International Max Planck Research School

MSc thesis with Prof. Dr. Ivo Feussner at the Albrechtvon-Haller-Institute of Plant Sciences, Georg August University Göttingen

MSc thesis title: The production of VLCPUFAs in algae and yeast

Degree: Master of Science

10/2003 - 09/2006

\section{BSc program Life Sciences}

Westphalian Wilhelm University Münster

Major subjects: Biochemistry, Molecular Biology

BSc thesis title: Analysis of inhibitors of the IKK/NF-kB signal module concerning their antiviral and cell toxic characteristics

Degree: Bachelor of Science

08/1994 - 06/2003 Goethe-Gymnasium (secondary school) Ibbenbüren Degree: University-entrance diploma 


\section{Presentations at International Conferences}

2010

2009

2008

Stipends

$10 / 2010-12 / 2010$

$10 / 2008-09 / 2010$

$10 / 2007-09 / 2008$

$10 / 2006-08 / 2007$

02/2006-03/2008
Poster at the $23^{\text {rd }}$ Conference of Plant Molecular Biology

(Dabringhausen, Germany)

Poster at the $4^{\text {th }}$ European Symposium on Plant Lipids (Göttingen, Germany)

Poster at the $7^{\text {th }}$ Euro Fed Lipid Congress

(Graz, Austria)

Oral presentation at the $6^{\text {th }}$ Euro Fed Lipid Congress

(Athens, Greece)

GGNB Bridging Fund

"Chemiefonds-Stipendium" from the "Verband der

Chemischen Industrie (VCI)"

Georg-Christoph-Lichtenberg-Stipend from the state

Lower Saxony

Stipend from the International Max Planck Research

School

Stipend from the German National Academic Foundation 